\title{
Fe(III)-Based Tandem Catalysis for Amidomethylative Multiple Substitution Reactions of $\alpha$-Substituted Styrene Derivatives
}

Xiaolin Qian, $\S$ Hui Zhou, $\S$ Hetti Handi Chaminda Lakmal, James Lucore, Xuesong Wang, Henry U. Valle, Bruno Donnadieu, Xue Xu, Xin Cui*

Department of Chemistry, Mississippi State University, Mississippi State, Mississippi 39762, United States

Corresponding Author *xcui@chemistry.msstate.edu

\section{Table of Contents}

1. General Considerations.

2. Optimization of Catalytic Amidomethylative Tandem Reactions of $\alpha$ Phenylstyrene S 4

3. General Procedure for $\mathrm{Fe}(\mathrm{OTf})_{3}$-Catalyzed Amidomethylative Tandem Reactions of $\alpha$-Phenylstyrene Derivatives with Bis(tosylamido)methane (B TM) S 5

4. Characterization of Products $3 a-q$ .S6

5. Large scale Synthesis of Representative Product 3a..............S16

6. Synthesis of Compound $4 a$. S16

7. Optimization of Catalytic Amidomethylative Tandem Reactions of $\alpha$ Methylstyrene

8. General Procedure for $\mathrm{Fe}(\mathrm{OTf})_{3}$-Catalyzed Amidomethylative Tandem Reactions of $\alpha$-Methylstyrene Derivatives with Bis(tosylamido)methane (BTM) 
9. Characterization of Products 6a-n............................................................

10. Large Scale Synthesis of Representative Product 6a.........S28

11. Mechanistic Study for $\mathbf{F e}(\mathrm{OTf})_{3}$-Catalyzed Amidomethylative Tandem

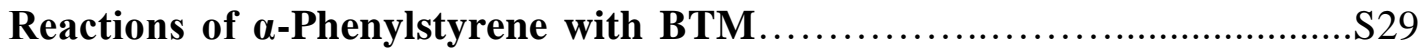

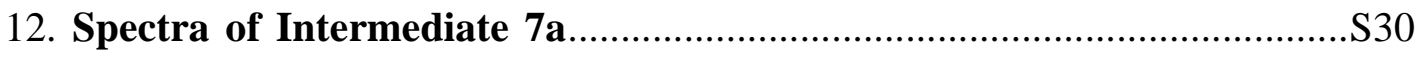

13. A Potential Alternative Pathway to the Amidomethylative Tandem Reactions

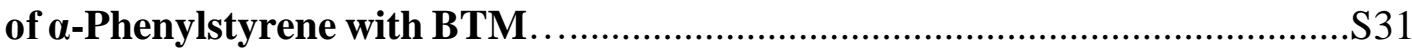

14. Mechanistic Study on Reaction Intermediate by HRMS

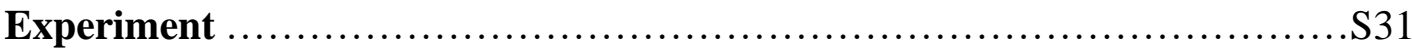

15. Mechanistic Study for Fe(OTf) 3 -Catalyzed Amidomethylative Tandem Reactions of $\alpha$-Methylstyrene with BTM................................ 33

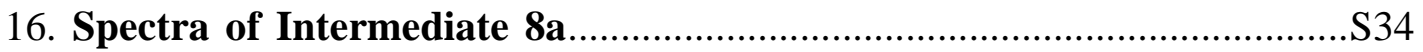

17. Deuterium Labeling Experiments...........................................................S34

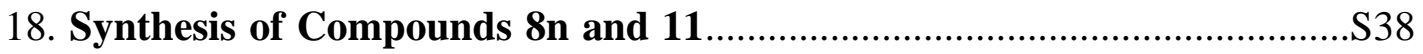

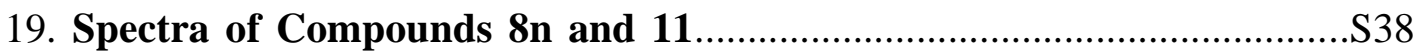

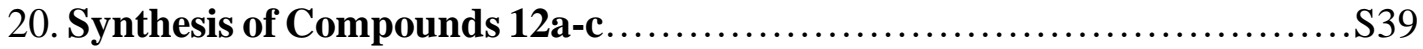

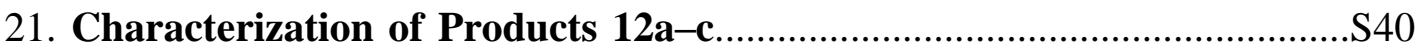

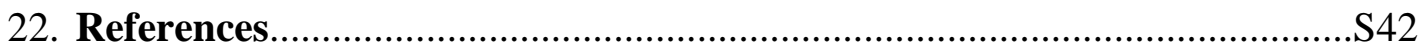

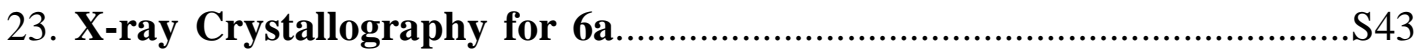

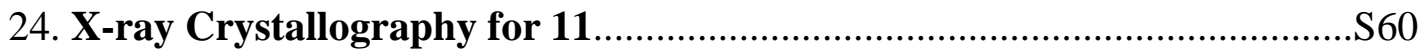

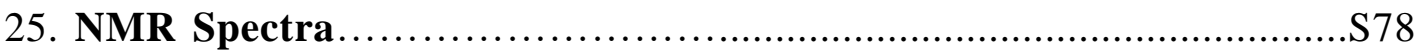




\section{General Considerations}

Experimental: Unless otherwise noted, all solvents were dried with sodium benzophenone and distilled before use. All reactions were set up under $\mathrm{N}_{2}$ atmosphere utilizing glassware that was oven-dried and cooled under vacuum. All non-aqueous manipulations were using standard Schlenk techniques. Reactions were monitored using thin-layer chromatography (TLC) on Silica Gel plates. Visualization of the developed plates was performed by UV light $(254 \mathrm{~nm})$ or $\mathrm{KMnO}_{4}$ stain. Silica-gel flash column chromatography was performed on Silicycle 40-63 $\mu \mathrm{m}$ silica gel.

Materials: Unless otherwise noted, starting catalysts and materials were obtained from Sigma-Aldrich, Oakwood Chemicals, Strem and Acros Co. Ltd. Moreover, commercially available reagents $1 \mathbf{a}$ and $\mathbf{5 a}$ were used without additional purification. Bis(tosylamido)methane ${ }^{1}$, styrene derivatives $\mathbf{1 h}-\mathbf{1 k}, \mathbf{1 n}-\mathbf{1 q}, \mathbf{5 b}-\mathbf{5} \mathbf{n}^{2}$, styrene derivatives $\mathbf{1 b}-\mathbf{1 g}, \mathbf{1 1}^{3}$ and $\mathbf{1} \mathbf{m}^{4}$ were prepared according to literature procedures.

The list of starting material-styrene derivatives:<smiles>C=C(c1ccccc1)c1ccccc1C(=C)c1ccccc1</smiles>

1b<smiles>C=C(c1ccccc1)c1cccc(C)c1</smiles>

$1 \mathrm{c}$

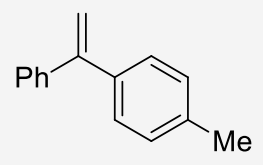

1d

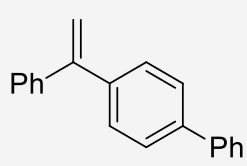

$1 e$<smiles>C=C(c1ccccc1)c1ccc2ccccc2c1</smiles>

$1 f$<smiles>C=C(c1ccccc1)c1ccc([N+](=O)[O-])cc1</smiles>

$1 \mathrm{k}$<smiles>C=C(c1ccc(Cl)cc1)c1ccc(Cl)cc1</smiles><smiles>C=C(c1ccccc1)c1ccc(OC)cc1</smiles>

$1 \mathrm{~g}$<smiles>C=C(c1ccccc1)c1ccc(C(F)(F)F)cc1</smiles>

1 I<smiles>C=C(c1ccccc1)c1ccccc1Cl</smiles>

$1 \mathrm{~h}$<smiles>C=C(c1ccccc1)c1ccc(Br)cc1</smiles>

$1 \mathrm{i}$

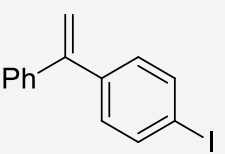

$1 \mathrm{j}$<smiles>C=C(c1ccccc1)c1cccc(C(F)(F)F)c1</smiles>

$1 \mathrm{~m}$<smiles>C=C(c1ccc(F)cc1)c1ccc(F)cc1</smiles>

$1 \mathrm{n}$<smiles>C=C(c1ccc(C(F)(F)F)cc1)c1ccc(C(F)(F)F)cc1</smiles>

$1 p$<smiles>C=C(c1ccc(F)cc1)c1ccc(OC)cc1</smiles>

$1 q$ 
<smiles>C=C(C)c1ccccc1</smiles>

$5 \mathbf{a}$<smiles>C=C(C)c1ccc(F)cc1</smiles>

$5 e$<smiles>C=C(C)c1cccc(Br)c1</smiles>

5j

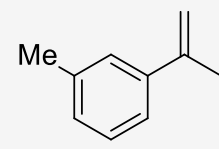

$5 b$<smiles>C=C(C)c1ccc(Br)cc1</smiles>

5c<smiles>C=C(C)c1ccc2ccccc2c1</smiles>

5d<smiles>C=C(C)c1cccc(Cl)c1</smiles>

$5 i$

Instrumentation: All NMR spectra were acquired from Bruker $300 \mathrm{MHz}, 500 \mathrm{MHz}$, or $600 \mathrm{MHz}$ in $\mathrm{CDCl}_{3}$ solution. ${ }^{1} \mathrm{H}$ NMR spectra were internally referenced to TMS. ${ }^{13} \mathrm{C}$ NMR spectra were internally referenced to the residual solvent signal. ${ }^{5}$ Data for ${ }^{1} \mathrm{H}$ NMR are reported as follows: chemical shift ( $\left.\delta \mathrm{ppm}\right)$, multiplicity ( $\mathrm{s}=$ singlet, $\mathrm{d}$ $=$ doublet, $\mathrm{t}=$ triplet, $\mathrm{q}=$ quartet, $\mathrm{m}=$ multiplet, $\mathrm{br}=$ broad $)$, coupling constants $(J)$ were reported in Hz. High Resolution Mass Spectra (HRMS) were recorded on Bruker MicrOTOF-QII mass instrument (ESI). Melting points of the solid compounds were determined on a Digimelt melting point apparatus. The crystals of compound 6a and 11 were mounted on the end of glass fibers, and the X-ray data were collected via a Bruker Kappa Apex II diffactometer.

\section{Optimization of Catalytic Amidomethylative Tandem Reactions of $\alpha$ - Phenylstyrene (including Table 1)}

General procedure for reaction optimization: An oven-dried Schlenk flask with a magnetic stir bar was charged with bis(tosylamido)methane $2(0.35 \mathrm{mmol})$ and catalyst (10 mol\%). Prior to coming into an inert atmosphere, vessels were further dried by purge-and-refill - the vessel is subjected to a vacuum to remove gases and water, and then refilled with $\mathrm{N}_{2}$ (repeated three times). Teflon screw cap was then replaced with a rubber septum and $\alpha$-phenylstyrene derivative $1 \mathrm{a}(0.1 \mathrm{mmol})$ was added, followed by the addition of $2 \mathrm{~mL}$ DCE. The resultant solution was purged with nitrogen for $1 \mathrm{~min}$, then equipped with the Teflon screw cap and stirred at indicated temperature for certain time showed in the table below. The crude mixture was 
evaporated via rota-vap to remove the solvent, and the yield of $\mathbf{3 a}$ was determined by ${ }^{1} \mathrm{H}$ NMR analysis with nitrobenzene as internal standard.

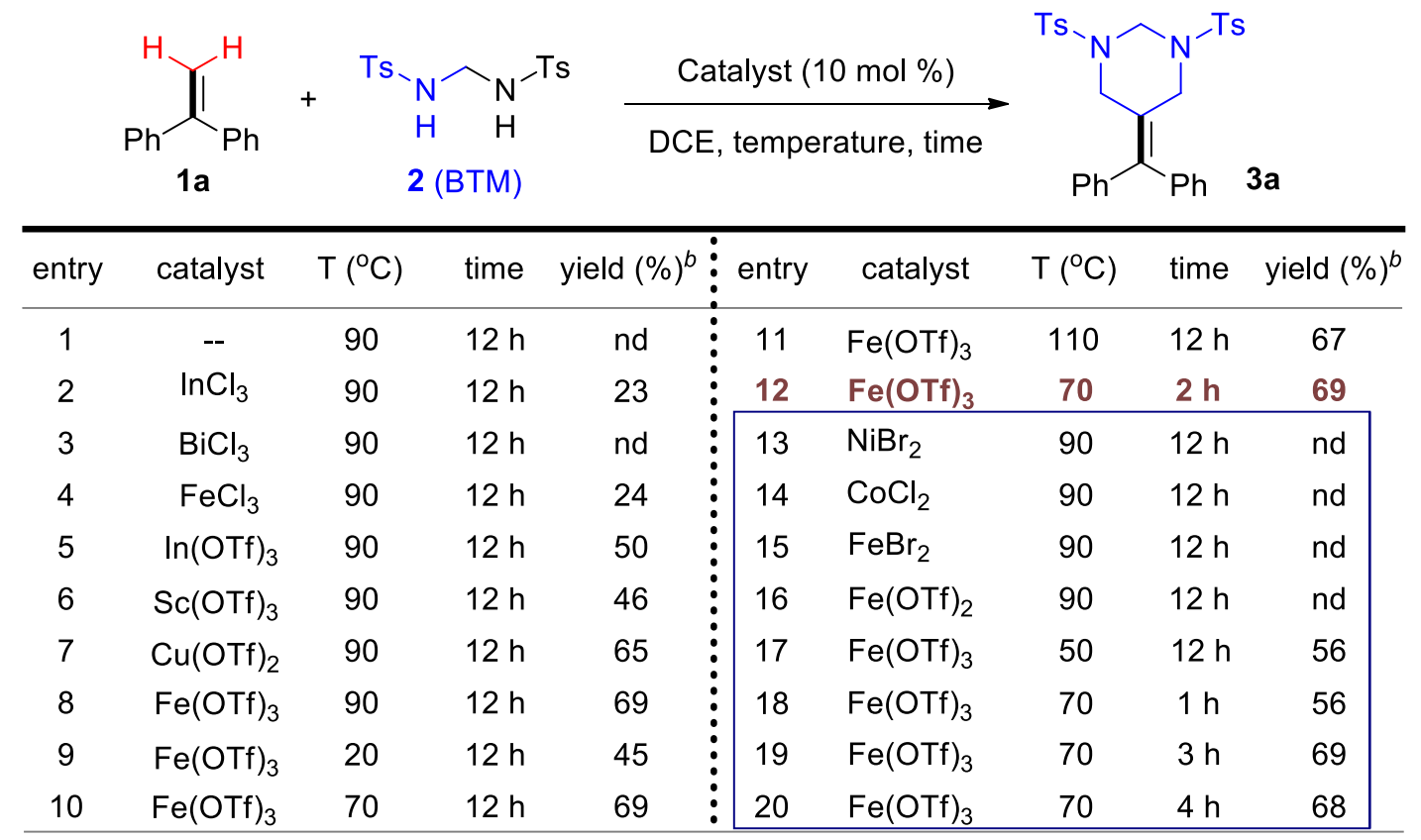

${ }^{a}$ Performed with 1a $(0.1 \mathrm{mmol}), 2(0.35 \mathrm{mmol})$, catalyst $(0.01 \mathrm{mmol})$ in $2.0 \mathrm{~mL}$ 1,2-dichloroethane (DCE). ${ }^{b 1} \mathrm{H}$ NMR yields.

\section{General Procedure for $\mathrm{Fe}(\mathrm{OTf})_{3}$-Catalyzed Amidomethylative Tandem} Reactions of $\alpha$-Phenylstyrene Derivatives with Bis(tosylamido)methane (BTM)

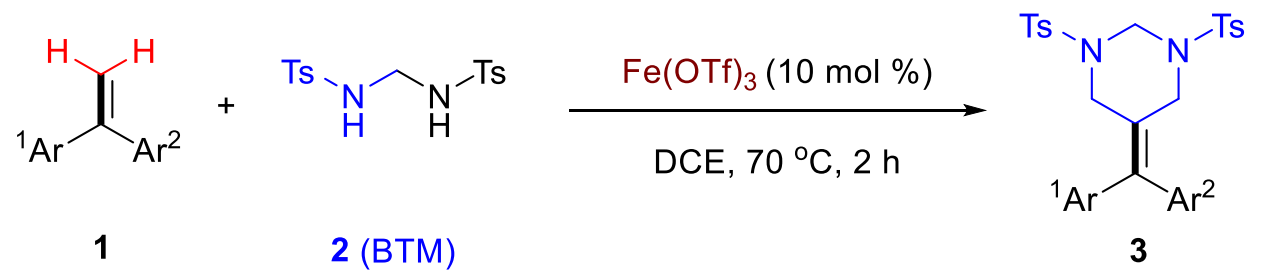

An oven-dried Schlenk flask with a magnetic stir bar was charged with bis(tosylamido)methane $(0.35 \mathrm{mmol})$ and catalyst $\mathrm{Fe}(\mathrm{OTf})_{3}(10 \mathrm{~mol} \%)$. Prior to coming into an inert atmosphere, vessels were further dried by purge-and-refill — the vessel is subjected to a vacuum to remove gases and water, and then refilled with $\mathrm{N}_{2}$ (repeated three times). Teflon screw cap was then replaced with a rubber septum and $\alpha$-phenylstyrene derivatives $(0.1 \mathrm{mmol})$ were added, followed by the addition of 2 mL DCE. The resultant solution was purged with nitrogen for $1 \mathrm{~min}$, then equipped with the Teflon screw cap and stirred at $70{ }^{\circ} \mathrm{C}$ for $2 \mathrm{~h}$. The reactions were monitored 
by TLC until the starting alkene was consumed. The crude mixture was purified by flash chromatography (hexane/ethyl acetate $=6: 1-3: 1$ ). The fractions containing the product were collected and concentrated by vacuum to afford the desired compounds 3a-q.

\section{Characterization of Products 3a-q}

\section{5-(diphenylmethylene)-1,3-ditosylhexahydropyrimidine}

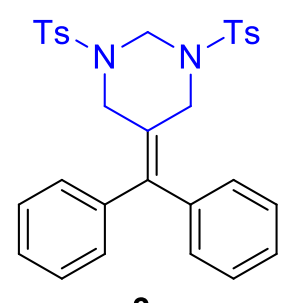

$3 a$

The title compound was prepared according to the general procedure as white solid (38.8 mg, 69\% yield, mp: $226.0-227.0{ }^{\circ} \mathrm{C}$ ). $\mathrm{R}_{f}=0.46$ (hexane/ethyl acetate $2: 1$ ). ${ }^{1} \mathbf{H}$ NMR $\left(500 \mathrm{MHz}, \mathrm{CDCl}_{3}\right) \delta 7.59(\mathrm{~d}, J=8.2 \mathrm{~Hz}, 4 \mathrm{H}), 7.31(\mathrm{~d}, J=8.2 \mathrm{~Hz}, 4 \mathrm{H}), 7.29-$ $7.25(\mathrm{~m}, 6 \mathrm{H}), 6.65-6.67(\mathrm{~m}, 4 \mathrm{H}), 4.82(\mathrm{~s}, 2 \mathrm{H}), 3.87(\mathrm{~s}, 4 \mathrm{H}), 2.44(\mathrm{~s}, 6 \mathrm{H}) ;{ }^{13} \mathrm{C}$ NMR (125 MHz, $\left.\mathrm{CDCl}_{3}\right) \delta 143.9$ (2 C), 142.9 (C), 139.5 (2 C), 134.9 (2 C), $129.9(4 \mathrm{CH})$, 129.7 (4 CH), 128.1 (4 CH), 128.0 (overlapping $4 \mathrm{CH}$ and $2 \mathrm{CH}$ ), $121.4(\mathrm{C}), 61.9$ $\left(\mathrm{CH}_{2}\right), 48.9\left(2 \mathrm{CH}_{2}\right), 21.5\left(2 \mathrm{CH}_{3}\right)$; HRMS (ESI) calcd. for $\mathrm{C}_{31} \mathrm{H}_{31} \mathrm{~N}_{2} \mathrm{O}_{4} \mathrm{~S}_{2}[\mathrm{M}+\mathrm{H}]$ : 559.1720, found: 559.1700.

\section{5-(phenyl(o-tolyl)methylene)-1,3-ditosylhexahydropyrimidine}<smiles>Cc1ccccc1C(=C1CN([13CH3])CN([13CH3])C1)c1ccccc1</smiles>

The title compound was prepared according to the general procedure as white solid (49.8 mg, 87\% yield, mp: $189.6-190.0{ }^{\circ} \mathrm{C}$ ). $\mathrm{R}_{f}=0.42$ (hexane/ethyl acetate $\left.2: 1\right) .{ }^{1} \mathbf{H}$ NMR $\left(500 \mathrm{MHz}, \mathrm{CDCl}_{3}\right) \delta 7.58(\mathrm{~d}, J=8.0 \mathrm{~Hz}, 2 \mathrm{H}), \delta 7.56(\mathrm{~d}, J=8.0 \mathrm{~Hz}, 2 \mathrm{H}), 7.33-$ 
$7.25(\mathrm{~m}, 7 \mathrm{H}), 7.18-7.15(\mathrm{~m}, 1 \mathrm{H}), 7.09-7.04(\mathrm{~m}, 2 \mathrm{H}), 6.83(\mathrm{dd}, J=7.5,1.5 \mathrm{~Hz}, 2 \mathrm{H})$, $6.30(\mathrm{~d}, J=7.5 \mathrm{~Hz}, 1 \mathrm{H}), 5.28(\mathrm{~d}, J=12.5 \mathrm{~Hz}, 1 \mathrm{H}), 4.40(\mathrm{~d}, J=15.3,1 \mathrm{H}), 4.35(\mathrm{~d}, J$ $=12.5,1 \mathrm{H}), 3.88(\mathrm{~d}, J=15.3 \mathrm{~Hz}, 1 \mathrm{H}), 3.75(\mathrm{~d}, J=13.7 \mathrm{~Hz}, 1 \mathrm{H}), 3.24(\mathrm{~d}, J=13.7$ $\mathrm{Hz}, 1 \mathrm{H}), 2.48$ (s, 3H), 2.43 (s, 3H), 1.75 (s, 3H); ${ }^{13} \mathbf{C}$ NMR (125 MHz, CDCl $) \delta 144.0$ (C), 143.8 (C), 141.7 (C), 138.7 (C), 138.5 (C), 135.9 (C), 135.8 (C), 133.9 (C), 130.4 $(\mathrm{CH}), 129.8(2 \mathrm{CH}), 129.7(2 \mathrm{CH}), 129.6(\mathrm{CH}), 129.4(2 \mathrm{CH}), 128.2(2 \mathrm{CH}), 128.1(2$ $\mathrm{CH}), 127.9(\mathrm{CH}), 127.8(\mathrm{CH}), 127.7(2 \mathrm{CH}), 125.6(\mathrm{CH}), 122.4(\mathrm{C}), 61.4\left(\mathrm{CH}_{2}\right), 48.6$ $\left(\mathrm{CH}_{2}\right), 47.4\left(\mathrm{CH}_{2}\right), 21.6\left(\mathrm{CH}_{3}\right), 21.5\left(\mathrm{CH}_{3}\right), 19.5\left(\mathrm{CH}_{3}\right)$; HRMS (ESI) calcd. for $\mathrm{C}_{32} \mathrm{H}_{32} \mathrm{KN}_{2} \mathrm{O}_{4} \mathrm{~S}_{2}$ [M+K]: 611.1435, found: 611.1433.

\section{5-(phenyl(m-tolyl)methylene)-1,3-ditosylhexahydropyrimidine}<smiles>Cc1cccc(C(=C2CN(C)CN([As])C2)c2ccccc2)c1</smiles>

The title compound was prepared according to the general procedure as light yellow solid (35.0 mg, $61 \%$ yield, $\mathrm{mp}: 238.5-239.6{ }^{\circ} \mathrm{C}$ ). $\mathrm{R}_{f}=0.51$ (hexane/ethyl acetate $2: 1$ ). ${ }^{1} \mathbf{H}$ NMR $\left(500 \mathrm{MHz}, \mathrm{CDCl}_{3}\right) \delta 7.60(\mathrm{~d}, J=8.0 \mathrm{~Hz}, 2 \mathrm{H}), 7.59(\mathrm{~d}, J=8.0 \mathrm{~Hz}, 2 \mathrm{H})$, 7.34-7.26 (m, 7H), 7.15 (t, $J=7.5 \mathrm{~Hz}, 1 \mathrm{H}), 7.09$ (d, $J=7.5 \mathrm{~Hz}, 1 \mathrm{H}), 6.70-6.68$ (m, 2H), 6.45-6.44 (m, 2H), 4.82 (s, 2H), 3.91 (s, 2H), 3.83 (s, 2H), 2.44 (s, 3H), 2.44 (s, 3H), 2.30 (s, 3H); ${ }^{13}$ C NMR (125 MHz, $\left.\mathrm{CDCl}_{3}\right) \delta 143.9$ (C), 143.8 (C), $143.0(\mathrm{C})$, 139.5 (C), 139.4 (C), 137.7 (C), 135.1 (C), 134.7 (C), 130.6 (CH), 129.9 (2 CH), $129.7(2 \mathrm{CH}), 129.6(2 \mathrm{CH}), 128.7(\mathrm{CH}), 128.1(\mathrm{CH}), 128.0$ (overlapping $2 \mathrm{CH}$ and $2 \mathrm{CH}), 127.9(2 \mathrm{CH}), 127.8(\mathrm{CH}), 126.9(\mathrm{CH}), 121.2(\mathrm{C}), 61.9\left(\mathrm{CH}_{2}\right), 49.0\left(\mathrm{CH}_{2}\right)$, $48.8\left(\mathrm{CH}_{2}\right), 21.5\left(\mathrm{CH}_{3}\right), 21.4\left(\mathrm{CH}_{3}\right), 21.2\left(\mathrm{CH}_{3}\right)$; HRMS (ESI) calcd. for $\mathrm{C}_{32} \mathrm{H}_{32} \mathrm{KN}_{2} \mathrm{O}_{4} \mathrm{~S}_{2}$ [M+K]: 611.1435, found: 611.1436.

\section{5-(phenyl(p-tolyl)methylene)-1,3-ditosylhexahydropyrimidine}




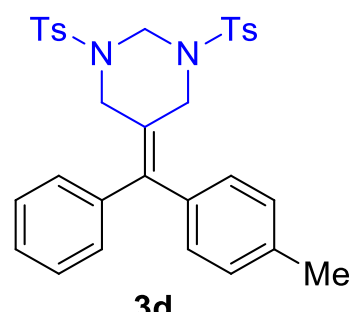

The title compound was prepared according to the general procedure as white solid (44.0 mg, 77\% yield, mp: $178.6-180.1^{\circ} \mathrm{C}$ ). $\mathrm{R}_{f}=0.50$ (hexane/ethyl acetate $2: 1$ ). ${ }^{1} \mathbf{H}$ NMR $\left(500 \mathrm{MHz}, \mathrm{CDCl}_{3}\right) \delta 7.59$ (t, $\left.J=8.4 \mathrm{~Hz}, 4 \mathrm{H}\right), 7.31-7.25(\mathrm{~m}, 7 \mathrm{H}), 7.08(\mathrm{~d}, J=$ $8.0 \mathrm{~Hz}, 2 \mathrm{H}), 6.66-6.62(\mathrm{~m}, 2 \mathrm{H}), 6.55$ (d, $J=8.0 \mathrm{~Hz}, 2 \mathrm{H}), 4.81$ (s, 2H), $3.90(\mathrm{~s}, 2 \mathrm{H})$, 3.84 (s, 2H), 2.44 (s, 6H), 2.34 (s, 3H); $\left.{ }^{13} \mathbf{C ~ N M R ~ ( 1 2 5 ~ M H z , ~ C D C l ~} 3\right) \delta 144.2(2 \mathrm{C})$, 143.1 (C), 139.9 (C), 138.1 (C), 136.8 (C), 135.2 (C), 135.1 (C), 130.2 (2 CH), 130.1 $(2 \mathrm{CH}), 130.0(2 \mathrm{CH}), 129.9(2 \mathrm{CH}), 129.1(2 \mathrm{CH}), 128.3$ (overlapping $2 \mathrm{CH}$ and 2 $\mathrm{CH}), 128.2(2 \mathrm{CH}), 128.1(\mathrm{CH}), 121.1(\mathrm{C}), 62.2\left(\mathrm{CH}_{2}\right), 49.3\left(\mathrm{CH}_{2}\right), 49.2\left(\mathrm{CH}_{2}\right), 21.8$ (overlapping $\mathrm{CH}_{3}$ and $\mathrm{CH}_{3}$ ), $21.4\left(\mathrm{CH}_{3}\right)$; HRMS (ESI) calcd. for $\mathrm{C}_{32} \mathrm{H}_{32} \mathrm{KN}_{2} \mathrm{O}_{4} \mathrm{~S}_{2}$ [M+Na]: 595.1696, found: 595.1697.

\section{5-([1,1'-biphenyl]-4-yl(phenyl)methylene)-1,3-ditosylhexahydropyrimidine}

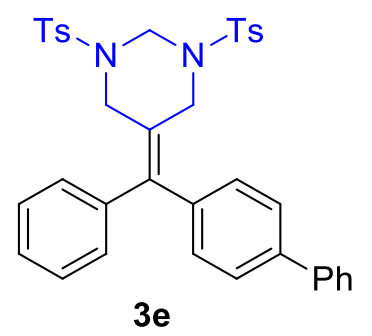

The title compound was prepared according to the general procedure as white solid (40.0 mg, 63\% yield, mp: 229.6-231.4 ${ }^{\circ} \mathrm{C}$ ). $\mathrm{R}_{f}=0.49$ (hexane/ethyl acetate 2:1). ${ }^{1} \mathbf{H}$ NMR $\left(500 \mathrm{MHz}, \mathrm{CDCl}_{3}\right) \delta 7.63-7.58(\mathrm{~m}, 6 \mathrm{H}), 7.50(\mathrm{~d}, J=8.2 \mathrm{~Hz}, 2 \mathrm{H}), 7.44$ (t, $J=$ $7.6 \mathrm{~Hz}, 2 \mathrm{H}), 7.35$ (t, $J=7.6 \mathrm{~Hz}, 1 \mathrm{H}), 7.33-7.28(\mathrm{~m}, 7 \mathrm{H}), 6.73(\mathrm{~d}, J=8.2 \mathrm{~Hz}, 2 \mathrm{H})$, 6.71-6.69 (m, 2H), 4.84 (s, 2H), 3.93 (s, 2H), 3.90 (s, 2H), 2.45 (s, 3H), 2.43 (s, 3H); ${ }^{13}$ C NMR (150 MHz, $\mathrm{CDCl}_{3}$ ) $\delta 144.3$ (C), 144.2 (C), 142.8 (C), 140.9 (C), 140.5 (C), 139.7 (C), 138.7 (C), 135.2 (C), 135.1 (C), 130.7 (2 CH), 130.3 (2 CH), $130.0(3 \mathrm{CH})$, $129.0(3 \mathrm{CH}), 128.4(2 \mathrm{CH}), 128.3(\mathrm{CH}), 128.2(4 \mathrm{CH}), 127.8(\mathrm{CH}), 127.2(2 \mathrm{CH})$, 
127.0 (2 $\mathrm{CH}), 121.8(\mathrm{C}), 62.2\left(\mathrm{CH}_{2}\right), 49.2\left(\right.$ overlapping $\mathrm{CH}_{2}$ and $\left.\mathrm{CH}_{2}\right), 21.8\left(\mathrm{CH}_{3}\right)$, $21.7\left(\mathrm{CH}_{3}\right)$; HRMS (ESI) calcd. for $\mathrm{C}_{37} \mathrm{H}_{35} \mathrm{~N}_{2} \mathrm{O}_{4} \mathrm{~S}_{2}[\mathrm{M}+\mathrm{H}]$ : 635.2033, found: 635.2034 .

\section{5-(naphthalen-2-yl(phenyl)methylene)-1,3-ditosylhexahydropyrimidine}

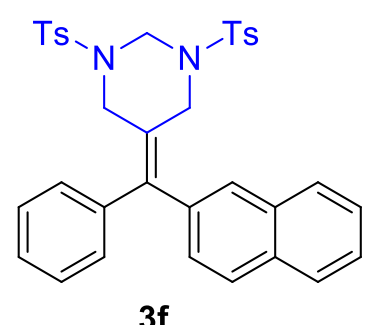

The title compound was prepared according to the general procedure as yellow solid (25.0 mg, 41\% yield, mp: $186.6-187.0^{\circ} \mathrm{C}$ ). $\mathrm{R}_{f}=0.43$ (hexane/ethyl acetate 2:1). ${ }^{1} \mathbf{H}$ NMR $\left(500 \mathrm{MHz}, \mathrm{CDCl}_{3}\right) \delta 7.81(\mathrm{dt}, J=6.5,3.3 \mathrm{~Hz}, 1 \mathrm{H}), 7.76-7.70(\mathrm{~m}, 2 \mathrm{H}), 7.65$ $(\mathrm{d}, J=8.2 \mathrm{~Hz}, 2 \mathrm{H}), 7.54(\mathrm{~d}, J=8.2 \mathrm{~Hz}, 2 \mathrm{H}), 7.50(\mathrm{dd}, J=6.5,3.3 \mathrm{~Hz}, 2 \mathrm{H}), 7.34(\mathrm{~d}$, $J=8.2 \mathrm{~Hz}, 2 \mathrm{H}), 7.31-7.30(\mathrm{~m}, 3 \mathrm{H}), 7.28(\mathrm{~d}, J=8.2 \mathrm{~Hz}, 2 \mathrm{H}), 7.07$ (s, 1H), 6.78-6.70 (m, 3H), 4.86 (s, 2H), 4.01 (s, 2H), 3.88 (s, 2H), 2.43 (s, 6H); ${ }^{13}$ C NMR (150 MHz, $\left.\mathrm{CDCl}_{3}\right) \delta 144.2(\mathrm{C}), 144.1$ (C), 143.1 (C), 139.5 (C), 136.9 (C), 135.5 (C), 134.6 (C), 132.9 (C), 132.8 (C), 130.3 (2 CH), $129.9(2 \mathrm{CH}), 129.8(2 \mathrm{CH}), 129.5(\mathrm{CH}), 128.4$ $(2 \mathrm{CH}), 128.3$ (overlapping $2 \mathrm{CH}$ and $\mathrm{CH}), 128.2(\mathrm{CH}), 128.0(2 \mathrm{CH}), 127.9(\mathrm{CH})$, 127.8 (CH), $127.7(\mathrm{CH}), 126.7(\mathrm{CH}), 126.6(\mathrm{CH}), 121.9(\mathrm{C}), 62.2\left(\mathrm{CH}_{2}\right), 49.4\left(\mathrm{CH}_{2}\right)$, $49.2\left(\mathrm{CH}_{2}\right), 21.7\left(\mathrm{CH}_{3}\right), 21.6\left(\mathrm{CH}_{3}\right)$; HRMS (ESI) calcd. for $\mathrm{C}_{35} \mathrm{H}_{32} \mathrm{NaN}_{2} \mathrm{O}_{4} \mathrm{~S}_{2}$ [M+Na]: 631.1696, found: 631.1699 .

\section{5-((4-methoxyphenyl)(phenyl)methylene)-1,3-ditosylhexahydropyrimidine}

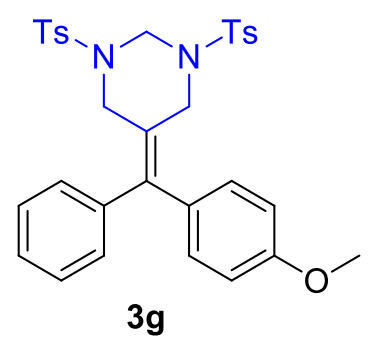

The title compound was prepared according to the general procedure as white solid (31.0 mg, 53\% yield, mp: $180.7-181.6^{\circ} \mathrm{C}$ ). $\mathrm{R}_{f}=0.43$ (hexane/ethyl acetate 2:1). ${ }^{1} \mathbf{H}$ 
$\operatorname{NMR}\left(500 \mathrm{MHz}, \mathrm{CDCl}_{3}\right) \delta 7.60(\mathrm{~d}, J=8.4 \mathrm{~Hz}, 2 \mathrm{H}), 7.58(\mathrm{~d}, J=8.4 \mathrm{~Hz}, 2 \mathrm{H}), 7.31-$ 7.29 (m, 4H), 7.28-7.25 (m, 3H), $6.80(\mathrm{~d}, J=8.7 \mathrm{~Hz}, 2 \mathrm{H}), 6.65-6.61(\mathrm{~m}, 2 \mathrm{H}), 6.59$ (d, $J=8.7 \mathrm{~Hz}, 2 \mathrm{H}), 4.81(\mathrm{~s}, 2 \mathrm{H}), 3.92(\mathrm{~s}, 2 \mathrm{H}), 3.83(\mathrm{~s}, 2 \mathrm{H}), 3.82(\mathrm{~s}, 3 \mathrm{H}), 2.44(\mathrm{~s}, 6 \mathrm{H})$; ${ }^{13}$ C NMR (125 MHz, CDCl $) \delta 159.2$ (C), 143.9 (C), 143.9 (C), 142.6 (C), 139.8 (C), $135.0(\mathrm{C}), 134.8(\mathrm{C}), 131.8(\mathrm{C}), 131.3(2 \mathrm{CH}), 130.0(2 \mathrm{CH}), 129.7(3 \mathrm{CH}), 129.6(3$ $\mathrm{CH}), 128.1(3 \mathrm{CH}), 128.0(2 \mathrm{CH}), 127.9(\mathrm{C}), 120.4(\mathrm{C}), 113.5(2 \mathrm{CH}), 62.0\left(\mathrm{CH}_{2}\right)$, $55.2\left(\mathrm{CH}_{3}\right), 49.1\left(\mathrm{CH}_{2}\right), 49.1\left(\mathrm{CH}_{2}\right), 21.6\left(\mathrm{CH}_{3}\right), 21.5\left(\mathrm{CH}_{3}\right) ; \mathrm{HRMS}$ (ESI) calcd. for $\mathrm{C}_{32} \mathrm{H}_{32} \mathrm{NaN}_{2} \mathrm{O}_{5} \mathrm{~S}_{2}$ [M+Na]: 611.1645, found: 611.1618.

\section{5-((2-chlorophenyl)(phenyl)methylene)-1,3-ditosylhexahydropyrimidine}<smiles>FC([AsH3])N1CC(=C(c2ccccc2)c2ccccc2Cl)CN([AsH2])C1</smiles>

The title compound was prepared according to the general procedure as white solid (55.0 mg, 92\% yield, mp: $179.0-180.8^{\circ} \mathrm{C}$ ). $\mathrm{R}_{f}=0.50$ (hexane/ethyl acetate $2: 1$ ). ${ }^{1} \mathbf{H}$ NMR $\left(500 \mathrm{MHz}, \mathrm{CDCl}_{3}\right) \delta 7.65(\mathrm{~d}, J=8.2 \mathrm{~Hz}, 2 \mathrm{H}), 7.61(\mathrm{~d}, J=8.2 \mathrm{~Hz}, 2 \mathrm{H}), 7.34$ (m, 6H), 7.31-7.30 (m, 2H), $7.26(\mathrm{td}, J=7.6,1.4 \mathrm{~Hz}, 1 \mathrm{H}), 7.21(\mathrm{t}, J=6.8 \mathrm{~Hz}, 1 \mathrm{H})$, $6.82(\mathrm{dd}, J=6.8,2.8 \mathrm{~Hz}, 2 \mathrm{H}), 6.52(\mathrm{dd}, J=7.6,1.4 \mathrm{~Hz}, 1 \mathrm{H}), 5.65(\mathrm{~d}, J=12.6 \mathrm{~Hz}$, $1 \mathrm{H}), 4.48(\mathrm{~d}, J=14.9 \mathrm{~Hz}, 1 \mathrm{H}), 4.05(\mathrm{~d}, J=12.6 \mathrm{~Hz}, 1 \mathrm{H}), 3.94(\mathrm{~d}, J=14.4 \mathrm{~Hz}, 1 \mathrm{H})$, 3.55 (d, $J=14.9 \mathrm{~Hz}, 1 \mathrm{H}), 3.34$ (d, $J=14.4 \mathrm{~Hz}, 1 \mathrm{H}), 2.50$ (s, 3H), 2.48 (s, 3H); ${ }^{13} \mathrm{C}$ NMR (125 MHz, $\left.\mathrm{CDCl}_{3}\right) \delta 144.3$ (C), 144.1 (C), 139.3 (C), 138.2 (C), 138.1 (C), $135.5(\mathrm{C}), 134.8(\mathrm{C}), 133.5(\mathrm{C}), 131.4(\mathrm{CH}), 130.1(\mathrm{CH}), 129.9$ (overlapping $2 \mathrm{CH}$ and $2 \mathrm{CH}), 129.6(\mathrm{CH}), 129.5(2 \mathrm{CH}), 128.4(2 \mathrm{CH}), 128.3(2 \mathrm{CH}), 128.2(\mathrm{CH}), 128.1$ $(2 \mathrm{CH}), 126.9(\mathrm{CH}), 124.3(\mathrm{C}), 61.6\left(\mathrm{CH}_{2}\right), 48.6\left(\mathrm{CH}_{2}\right), 47.8\left(\mathrm{CH}_{2}\right), 21.8$ (overlapping $\mathrm{CH}_{3}$ and $\mathrm{CH}_{3}$ ); HRMS (ESI) calcd. for $\mathrm{C}_{31} \mathrm{H}_{29} \mathrm{ClKN}_{2} \mathrm{O}_{4} \mathrm{~S}_{2}[\mathrm{M}+\mathrm{K}]: 631.0889$, found: 631.0884 .

\section{5-((4-bromophenyl)(phenyl)methylene)-1,3-ditosylhexahydropyrimidine}




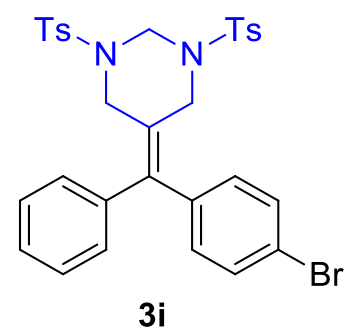

The title compound was prepared according to the general procedure as white solid (58.0 mg, $91 \%$ yield, mp: $178.6-180.5^{\circ} \mathrm{C}$ ). $\mathbf{R}_{f}=0.48$ (hexane/ethyl acetate 2:1). ${ }^{1} \mathbf{H}$ NMR $\left(500 \mathrm{MHz}, \mathrm{CDCl}_{3}\right) \delta 7.58(\mathrm{~d}, J=8.2 \mathrm{~Hz}, 4 \mathrm{H}), 7.39(\mathrm{~d}, J=8.2 \mathrm{~Hz}, 2 \mathrm{H}), 7.30$ (m, 7H), 6.69-6.64 (m, 2H), 6.49 (d, J = 8.3 Hz, 2H), 4.83 (s, 2H), 3.91 (s, 2H), 3.79 (s, 2H), 2.44 (s, 6H); ${ }^{13} \mathrm{C}$ NMR (125 MHz, $\mathrm{CDCl}_{3}$ ) $\delta 144.2$ (C), 144.1 (C), 141.8 (C), $139.0(\mathrm{C}), 138.5(\mathrm{C}), 135.2(\mathrm{C}), 134.6(\mathrm{C}), 131.6(2 \mathrm{CH}), 131.4(2 \mathrm{CH}), 130.0(2 \mathrm{CH})$, $129.8(2 \mathrm{CH}), 129.7(2 \mathrm{CH}), 128.4(\mathrm{CH}), 128.3(2 \mathrm{CH}), 128.2(2 \mathrm{CH}), 128.0(2 \mathrm{CH})$, $122.3(\mathrm{C}), 122.2(\mathrm{C}), 62.0\left(\mathrm{CH}_{2}\right), 49.0\left(\mathrm{CH}_{2}\right), 48.9\left(\mathrm{CH}_{2}\right), 21.7\left(\mathrm{CH}_{3}\right), 21.6\left(\mathrm{CH}_{3}\right)$; HRMS (ESI) calcd. for $\mathrm{C}_{31} \mathrm{H}_{29} \mathrm{BrNaN}_{2} \mathrm{O}_{4} \mathrm{~S}_{2}$ [M+Na]: 659.0644, found: 659.0645 .

\section{5-((4-iodophenyl)(phenyl)methylene)-1,3-ditosylhexahydropyrimidine}

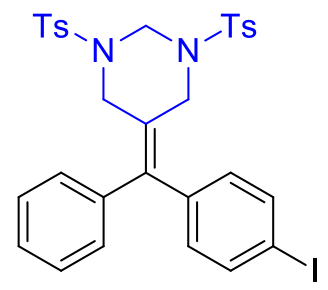

3j

The title compound was prepared according to the general procedure as white solid (57.0 mg, 83\% yield, mp: $172.2-173.8^{\circ} \mathrm{C}$ ). $\mathrm{R}_{f}=0.49$ (hexane/ethyl acetate 2:1). ${ }^{1} \mathbf{H}$ NMR $\left(500 \mathrm{MHz}, \mathrm{CDCl}_{3}\right) \delta 7.59(\mathrm{~d}, J=8.0 \mathrm{~Hz}, 6 \mathrm{H}), 7.29(\mathrm{~m}, 7 \mathrm{H}), 6.69-6.63(\mathrm{~m}, 2 \mathrm{H})$, $6.36(\mathrm{~d}, J=8.0 \mathrm{~Hz}, 2 \mathrm{H}), 4.82$ (s, 2H), 3.91 (s, 2H), 3.79 (s, 2H), 2.45 (s, 6H); ${ }^{13} \mathrm{C}$ NMR (125 MHz, CDCl $\left.{ }_{3}\right) \delta 144.2$ (C), 144.0 (C), 141.9 (C), 139.1 (C), 138.9 (C), $137.4(2 \mathrm{CH}), 135.2(\mathrm{C}), 134.6(\mathrm{C}), 131.8(2 \mathrm{CH}), 130.0(2 \mathrm{CH}), 129.8(2 \mathrm{CH}), 129.7$ $(2 \mathrm{CH}), 128.4(\mathrm{CH}), 128.3(2 \mathrm{CH}), 128.2(2 \mathrm{CH}), 128.0(2 \mathrm{CH}), 122.2(\mathrm{C}), 94.1(\mathrm{C})$, $61.9\left(\mathrm{CH}_{2}\right), 49.0\left(\mathrm{CH}_{2}\right), 48.9\left(\mathrm{CH}_{2}\right), 21.7\left(\mathrm{CH}_{3}\right), 21.6\left(\mathrm{CH}_{3}\right)$; HRMS (ESI) calcd. for $\mathrm{C}_{31} \mathrm{H}_{30} \mathrm{IN}_{2} \mathrm{O}_{4} \mathrm{~S}_{2}[\mathrm{M}+\mathrm{H}]:$ 685.0686, found: 685.0682 . 


\section{5-((4-nitrophenyl)(phenyl)methylene)-1,3-ditosylhexahydropyrimidine}<smiles>[13CH3]N1CC(=C(c2ccccc2)c2ccc([N+](=O)[O-])cc2)CN([AsH3])C1</smiles>

The title compound was prepared according to the general procedure as white solid (57.8 mg, 95\% yield, mp: $131.2-133.1^{\circ} \mathrm{C}$ ). $\mathrm{R}_{f}=0.32$ (hexane/ethyl acetate $2: 1$ ). ${ }^{1} \mathbf{H}$ NMR $\left(500 \mathrm{MHz}, \mathrm{CDCl}_{3}\right)$ 1H NMR $\left(500 \mathrm{MHz} \mathrm{CDCl}_{3}\right) \delta 8.11(\mathrm{~d}, J=8.6 \mathrm{~Hz}, 2 \mathrm{H})$, $7.62(\mathrm{~d}, J=8.2 \mathrm{~Hz}, 2 \mathrm{H}), 7.57(\mathrm{~d}, J=8.2 \mathrm{~Hz}, 2 \mathrm{H}), 7.34-7.31(\mathrm{~m}, 7 \mathrm{H}), 6.77(\mathrm{~d}, J=$ $8.6 \mathrm{~Hz}, 2 \mathrm{H}), 6.74-6.67(\mathrm{~m}, 2 \mathrm{H}), 4.85(\mathrm{~s}, 2 \mathrm{H}), 4.00(\mathrm{~s}, 2 \mathrm{H}), 3.73(\mathrm{~s}, 2 \mathrm{H}), 2.47(\mathrm{~s}, 3 \mathrm{H})$, 2.45 (s, 3H); ${ }^{13} \mathrm{C}$ NMR (125 MHz, $\left.\mathrm{CDCl}_{3}\right) \delta 147.5$ (C), 146.4 (C), 144.6 (C), 144.3 (C), 141.1 (C), 138.4 (C), 135.6 (C), 134.4 (C), 131.0 (2 CH), 130.1 (2 CH), 130.1 (2 $\mathrm{CH}), 129.9(2 \mathrm{CH}), 129.0(\mathrm{CH}), 128.8(2 \mathrm{CH}), 128.4(2 \mathrm{CH}), 128.0(2 \mathrm{CH}), 124.4$ (C), $123.6(2 \mathrm{CH}), 62.0\left(\mathrm{CH}_{2}\right), 49.1\left(\mathrm{CH}_{2}\right), 48.8\left(\mathrm{CH}_{2}\right), 21.8\left(\mathrm{CH}_{3}\right), 21.7\left(\mathrm{CH}_{3}\right)$; HRMS (ESI) calcd. for $\mathrm{C}_{31} \mathrm{H}_{30} \mathrm{~N}_{3} \mathrm{O}_{6} \mathrm{~S}_{2}[\mathrm{M}+\mathrm{H}]$ : 604.1571, found: 604.1573.

\section{5-(phenyl(4-(trifluoromethyl)phenyl)methylene)-1,3-}

\section{ditosylhexahydropyrimidine}<smiles>FC(F)(F)c1ccc(C(=C2CN([As])CN([As])C2)c2ccccc2)cc1</smiles>

The title compound was prepared according to the general procedure as white solid (62.6 mg, 99\% yield, mp: $187.2-188.0{ }^{\circ} \mathrm{C}$ ). $\mathrm{R}_{f}=0.42$ (hexane/ethyl acetate $\left.2: 1\right) .{ }^{1} \mathbf{H}$ NMR $\left(500 \mathrm{MHz}, \mathrm{CDCl}_{3}\right) \delta 7.60(\mathrm{~d}, J=8.2 \mathrm{~Hz}, 2 \mathrm{H}), 7.57(\mathrm{~d}, J=8.2 \mathrm{~Hz}, 2 \mathrm{H}), 7.51$ $(\mathrm{d}, J=8.0 \mathrm{~Hz}, 2 \mathrm{H}), 7.36-7.28(\mathrm{~m}, 7 \mathrm{H}), 6.75(\mathrm{~d}, J=8.0 \mathrm{~Hz}, 2 \mathrm{H}), 6.69(\mathrm{dd}, J=6.4$, $2.9 \mathrm{~Hz}, 2 \mathrm{H}), 4.85$ (s, 2H), $3.96(\mathrm{~s}, 2 \mathrm{H}), 3.77$ (s, 2H), $2.45(\mathrm{~s}, 3 \mathrm{H}), 2.44(\mathrm{~s}, 3 \mathrm{H}) ;{ }^{13} \mathrm{C}$ NMR (125 MHz, $\left.\mathrm{CDCl}_{3}\right) \delta 144.3$ (C), 144.1 (C), 143.2 (C), 141.6 (C), 138.7 (C), 
$135.3(\mathrm{C}), 134.5(\mathrm{C}), 130.0(2 \mathrm{CH}), 129.8$ (q, $J=33.2 \mathrm{~Hz})(\mathrm{C}), 129.9(2 \mathrm{CH}), 129.8$ $(2 \mathrm{CH}), 129.7(2 \mathrm{CH}), 128.5(\mathrm{CH}), 128.4(2 \mathrm{CH}), 128.2(2 \mathrm{CH}), 127.9(2 \mathrm{CH}), 125.1(\mathrm{q}$, $J=3.6 \mathrm{~Hz})(2 \mathrm{CH}), 124.1(\mathrm{q}, J=270.9 \mathrm{~Hz})\left(\mathrm{CF}_{3}\right), 123.1(\mathrm{C}), 61.8\left(\mathrm{CH}_{2}\right), 48.8\left(\mathrm{CH}_{2}\right)$, $48.7\left(\mathrm{CH}_{2}\right), 21.6\left(\mathrm{CH}_{3}\right), 21.5\left(\mathrm{CH}_{3}\right) ;{ }^{19} \mathbf{F}$ NMR $\left(470 \mathrm{MHz}, \mathrm{CDCl}_{3}\right) \delta-63.29 ;$ HRMS (ESI) calcd. for $\mathrm{C}_{32} \mathrm{H}_{29} \mathrm{~F}_{3} \mathrm{KN}_{2} \mathrm{O}_{4} \mathrm{~S}_{2}$ [M+K]: 665.1152, found: 665.1139.

\section{5-(phenyl(3-(trifluoromethyl)phenyl)methylene)-1,3-}

\section{ditosylhexahydropyrimidine}

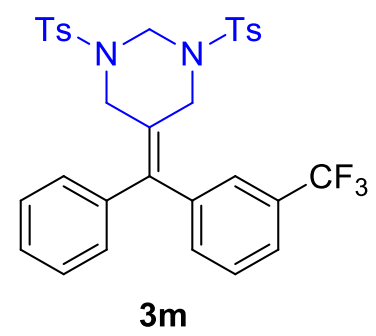

The title compound was prepared according to the general procedure as white solid (45.2 mg, 72\% yield, mp: 206.9-208.6 ${ }^{\circ} \mathrm{C}$ ). $\mathrm{R}_{f}=0.48$ (hexane/ethyl acetate 2:1). ${ }^{1} \mathbf{H}$ NMR $\left(500 \mathrm{MHz}, \mathrm{CDCl}_{3}\right) \delta 7.60-7.55(\mathrm{~m}, 5 \mathrm{H}), 7.41(\mathrm{t}, J=7.5 \mathrm{~Hz}, 1 \mathrm{H}), 7.32-7.31$ (m, 7H), $6.90(\mathrm{~s}, 1 \mathrm{H}), 6.83(\mathrm{~d}, J=7.5 \mathrm{~Hz}, 1 \mathrm{H}), 6.68-6.67$ (m, 2H), 4.84 (s, 2H), 3.95 (s, 2H), 3.76 (s, 2H), 2.44 (s, 6H).; ${ }^{13}$ C NMR (125 MHz, $\left.\mathrm{CDCl}_{3}\right) \delta 144.3(\mathrm{C}), 144.2$ (C), 141.5 (C), 140.4 (C), 138.6 (C), 135.2 (C), 134.6 (C), 133.3 (CH), 130.7 (q, J = $32.4 \mathrm{~Hz}$ ) (C), $129.9(2 \mathrm{CH}), 129.8(2 \mathrm{CH}), 129.7(2 \mathrm{CH}), 128.8(\mathrm{CH}), 128.5(\mathrm{CH})$, $128.4(2 \mathrm{CH}), 128.1(2 \mathrm{CH}), 127.9(2 \mathrm{CH}), 126.5(\mathrm{q}, J=4.6 \mathrm{~Hz})(\mathrm{CH}), 124.9(\mathrm{q}, J$ $=3.1 \mathrm{~Hz})(\mathrm{CH}), 123.1(\mathrm{C}), 124.0(\mathrm{q}, J=270.3 \mathrm{~Hz})\left(\mathrm{CF}_{3}\right), 61.9\left(\mathrm{CH}_{2}\right), 48.8\left(\mathrm{CH}_{2}\right)$, $48.7\left(\mathrm{CH}_{2}\right), 21.6\left(\mathrm{CH}_{3}\right), 21.5\left(\mathrm{CH}_{3}\right) . ;{ }^{19} \mathbf{F}$ NMR $\left(470 \mathrm{MHz}, \mathrm{CDCl}_{3}\right) \delta-62.51$; HRMS (ESI) calcd. for $\mathrm{C}_{32} \mathrm{H}_{29} \mathrm{~F}_{3} \mathrm{NaN}_{2} \mathrm{O}_{4} \mathrm{~S}_{2}$ [M+Na]: 649.1413, found: 649.1412.

\section{5-(bis(4-fluorophenyl)methylene)-1,3-ditosylhexahydropyrimidine}<smiles>Fc1ccc(C(=C2CN([As])CN([As])C2)c2ccc(F)cc2)cc1</smiles>

$3 n$ 
The title compound was prepared according to the general procedure as white solid (54.8 mg, 92\% yield, mp: $187.4-188.0^{\circ} \mathrm{C}$ ). $\mathrm{R}_{f}=0.44$ (hexane/ethyl acetate $2: 1$ ). ${ }^{1} \mathbf{H}$ NMR $\left(500 \mathrm{MHz}, \mathrm{CDCl}_{3}\right) \delta 7.59(\mathrm{~d}, J=8.2 \mathrm{~Hz}, 4 \mathrm{H}), 7.31(\mathrm{~d}, J=8.2 \mathrm{~Hz}, 4 \mathrm{H}), 6.98(\mathrm{t}$, $J=8.6 \mathrm{~Hz}, 4 \mathrm{H}), 6.66-6.58(\mathrm{~m}, 4 \mathrm{H}), 4.83(\mathrm{~s}, 2 \mathrm{H}), 3.85$ (s, 4H), 2.44 (s, 6H); ${ }^{13} \mathbf{C}$ NMR $\left(125 \mathrm{MHz}, \mathrm{CDCl}_{3}\right) \delta 162.4$ (d, J=249.4 Hz) (2 C-F), $144.1(2 \mathrm{C}), 140.8(\mathrm{C}), 135.3$ $(\mathrm{d}, J=3.4 \mathrm{~Hz})(2 \mathrm{C}), 134.9(2 \mathrm{C}), 131.7(\mathrm{~d}, J=8.2 \mathrm{~Hz})(4 \mathrm{CH}), 129.7(4 \mathrm{CH}), 128.0$ $(4 \mathrm{CH}), 122.0(\mathrm{C}), 115.3(\mathrm{~d}, J=21.7 \mathrm{~Hz})(4 \mathrm{CH}), 61.9\left(\mathrm{CH}_{2}\right), 48.9\left(2 \mathrm{CH}_{2}\right), 21.5(2$ $\left.\mathrm{CH}_{3}\right) ;{ }^{19} \mathbf{F}$ NMR $\left(470 \mathrm{MHz}, \mathrm{CDCl}_{3}\right) \delta-113.45$; HRMS (ESI) calcd. for $\mathrm{C}_{31} \mathrm{H}_{28} \mathrm{~F}_{2} \mathrm{KN}_{2} \mathrm{O}_{4} \mathrm{~S}_{2}$ [M+K]: 633.1090, found: 633.1080 .

\section{5-(bis(4-chlorophenyl)methylene)-1,3-ditosylhexahydropyrimidine}<smiles>Clc1ccc(C(=C2CN([As])CN([As])C2)c2ccc(Cl)cc2)cc1</smiles>

The title compound was prepared according to the general procedure as white solid (50.0 mg, 80\% yield, mp: $229.2-230.7^{\circ} \mathrm{C}$ ). $\mathrm{R}_{f}=0.50$ (hexane/ethyl acetate 2:1). ${ }^{1} \mathbf{H}$ NMR $\left(500 \mathrm{MHz}, \mathrm{CDCl}_{3}\right) \delta 7.58(\mathrm{~d}, J=8.1 \mathrm{~Hz}, 4 \mathrm{H}), 7.31(\mathrm{~d}, J=8.1 \mathrm{~Hz}, 4 \mathrm{H}), 7.26$ (d, $J=8.2 \mathrm{~Hz}, 4 \mathrm{H}), 6.57$ (d, $J=8.2 \mathrm{~Hz}, 4 \mathrm{H}), 4.83$ (s, 2H), 3.84 (s, 4H), 2.45 (s, 6H); ${ }^{13} \mathrm{C}$ NMR (125 MHz, $\left.\mathrm{CDCl}_{3}\right) \delta 144.4$ (2 C), 140.8 (C), 137.6 (2 C), 135.0 (2 C), $134.5(2 \mathrm{C}), 131.5(4 \mathrm{CH}), 129.9(4 \mathrm{CH}), 128.8(4 \mathrm{CH}), 128.2(4 \mathrm{CH}), 123.0(\mathrm{C}), 62.1$ $\left(\mathrm{CH}_{2}\right), 49.1\left(2 \mathrm{CH}_{2}\right), 21.8\left(2 \mathrm{CH}_{3}\right)$; HRMS (ESI) calcd. for $\mathrm{C}_{31} \mathrm{H}_{28} \mathrm{Cl}_{2} \mathrm{KN}_{2} \mathrm{O}_{4} \mathrm{~S}_{2}$ [M+K]: 665.0499, found: 665.0491.

\section{5-(bis(4-(trifluoromethyl)phenyl)methylene)-1,3-ditosylhexahydropyrimidine}




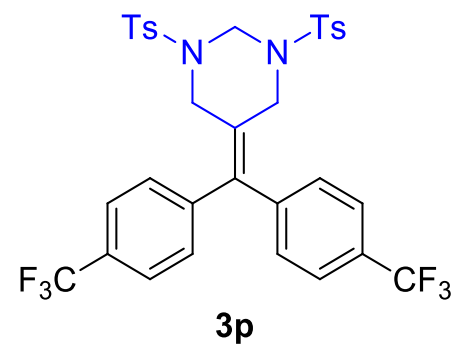

The title compound was prepared according to the general procedure as light yellow solid (37 mg, 53\% yield, mp: 234.0-235.8 ${ }^{\circ} \mathrm{C}$ ). $\mathrm{R}_{f}=0.38$ (hexane/ethyl acetate 2:1). ${ }^{1} \mathbf{H}$ NMR $\left(500 \mathrm{MHz}, \mathrm{CDCl}_{3}\right) \delta 7.60(\mathrm{~d}, J=8.2 \mathrm{~Hz}, 4 \mathrm{H}), 7.56(\mathrm{~d}, J=8.1 \mathrm{~Hz}, 4 \mathrm{H}), 7.32$ (d, $J=8.2 \mathrm{~Hz}, 4 \mathrm{H}), 6.79$ (d, $J=8.1 \mathrm{~Hz}, 4 \mathrm{H}), 4.86$ (s, 2H), 3.86 (s, 4H), 2.46 (s, 6H); ${ }^{13} \mathrm{C}$ NMR (125 MHz, $\left.\mathrm{CDCl}_{3}\right) \delta 144.3$ (2 C) , 142.3(2 C), 140.2 (C), 134.8 (2 C), $130.5(\mathrm{q}, J=32.6 \mathrm{~Hz})(2 \mathrm{C}), 130.3(4 \mathrm{CH}), 129.8(4 \mathrm{CH}), 128.1(4 \mathrm{CH}), 125.5(\mathrm{q}, J$ $=3.4 \mathrm{~Hz})(4 \mathrm{CH}), 124.9(\mathrm{C}), 123.8(\mathrm{q}, J=270.8 \mathrm{~Hz})\left(2 \mathrm{CF}_{3}\right), 61.7\left(\mathrm{CH}_{2}\right), 48.7(2$ $\left.\mathrm{CH}_{2}\right), 21.6\left(2 \mathrm{CH}_{3}\right) ;{ }^{19} \mathbf{F}$ NMR (470 MHz, $\left.\mathrm{CDCl}_{3}\right) \delta-63.38$; HRMS (ESI) calcd. for $\mathrm{C}_{33} \mathrm{H}_{28} \mathrm{~F}_{6} \mathrm{KN}_{2} \mathrm{O}_{4} \mathrm{~S}_{2}[\mathrm{M}+\mathrm{K}]$ : 733.1026, found: 733.1014.

\section{5-((4-fluorophenyl)(4-methoxyphenyl)methylene)-1,3-}

ditosylhexahydropyrimidine

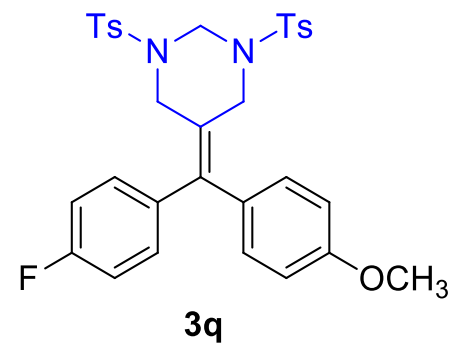

The title compound was prepared according to the general procedure as white solid (31.2 mg, 51\% yield, mp: $181.2-182.5^{\circ} \mathrm{C}$ ). $\mathrm{R}_{f}=0.47$ (hexane/ethyl acetate $2: 1$ ). ${ }^{1} \mathbf{H}$ NMR $\left(500 \mathrm{MHz}, \mathrm{CDCl}_{3}\right) \delta 7.60(\mathrm{~d}, J=8.2 \mathrm{~Hz}, 2 \mathrm{H}), 7.58(\mathrm{~d}, J=8.2 \mathrm{~Hz}, 2 \mathrm{H}), 7.30$ (d, $J=8.2 \mathrm{~Hz}, 4 \mathrm{H}), 6.95$ (t, $J=8.6 \mathrm{~Hz}, 2 \mathrm{H}), 6.82(\mathrm{~d}, J=8.8 \mathrm{~Hz}, 2 \mathrm{H}), 6.60$ (d, $J=8.8$ Hz, 2H), 6.59-6.55 (m, 2H), 4.82 (s, 2H), 3.95 (s, 2H), 3.83 (s, 3H), 3.77 (s, 2H), 2.44 (s, 6H); ${ }^{13} \mathrm{C}$ NMR $\left(125 \mathrm{MHz}, \mathrm{CDCl}_{3}\right) \delta 162.5(\mathrm{~d}, J=246.8 \mathrm{~Hz})(\mathrm{C}-\mathrm{F}), 159.6(\mathrm{C})$, 144.2 (C), 144.1 (C), 141.8 (C), 135.9 (d, J = 4.0 Hz) (C), 135.5 (C), 134.8 (C), 131.9 $(\mathrm{d}, J=8.1 \mathrm{~Hz})(2 \mathrm{CH}), 131.8(\mathrm{C}), 131.5(2 \mathrm{CH}), 129.9(2 \mathrm{CH}), 129.8(2 \mathrm{CH}), 128.4$ 
(2 CH), $128.1(2 \mathrm{CH}), 120.9(\mathrm{C}), 115.3(\mathrm{~d}, J=21.4 \mathrm{~Hz})(2 \mathrm{CH}), 113.8(2 \mathrm{CH}), 62.3$ $\left(\mathrm{CH}_{2}\right), 55.5\left(\mathrm{OCH}_{3}\right), 49.4\left(\mathrm{CH}_{2}\right), 49.3\left(\mathrm{CH}_{2}\right), 21.8$ (overlapping $\mathrm{CH}_{3}$ and $\left.\mathrm{CH}_{3}\right) ;{ }^{19} \mathbf{F}$ NMR $\left(470 \mathrm{MHz}, \mathrm{CDCl}_{3}\right) \delta-114.03$; HRMS (ESI) calcd. for $\mathrm{C}_{32} \mathrm{H}_{31} \mathrm{KN}_{2} \mathrm{O}_{5} \mathrm{~S}_{2}[\mathrm{M}+\mathrm{K}]$ : 649.1290, found: 649.1292 .

\section{Large Scale (gram) Synthesis of Representative Product 3a}

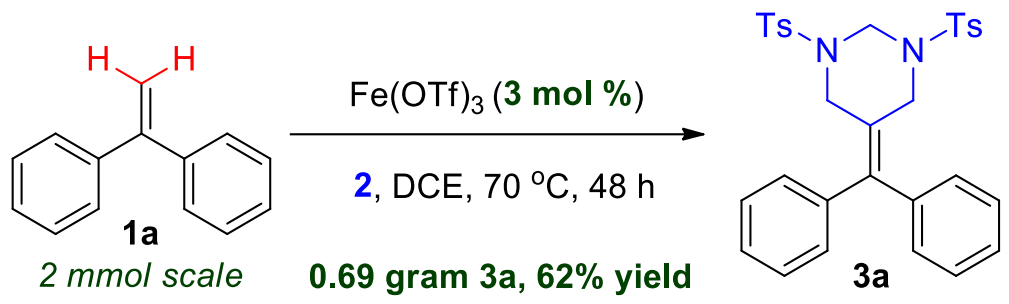

According to the general procedure with minor modifications: an oven-dried $75 \mathrm{~mL}$ Schlenk tube was charged with bis(tosylamido)methane (7 mmol), catalyst Fe(OTf) 3 ( $3 \mathrm{~mol} \%$ ) and a magnetic stir bar, then evacuated and back filled with $\mathrm{N}_{2}$ for three times. 1,1-Diphenylethylene (2 mmol) and $30 \mathrm{~mL}$ DCE was added after replacement of the Teflon screw cap with a rubber septum. The Schlenk tube was then purged with nitrogen for 1 min and the rubber septum was again replaced with the Teflon screw cap. The reaction mixture was stirred at $70^{\circ} \mathrm{C}$ for $48 \mathrm{~h}$. After the reaction is done, the crude mixture was purified by flash chromatography (hexane/ethyl acetate $=6: 1-3: 1$ ). The fractions containing the product were collected and concentrated by vacuum to afford the desired product 3a (0.69 g, 62\% yield).

\section{Synthesis of Compound 4a}

N,N'-(2-(diphenylmethylene)propane-1,3-diyl)bis(4-methylbenzenesulfonamide)

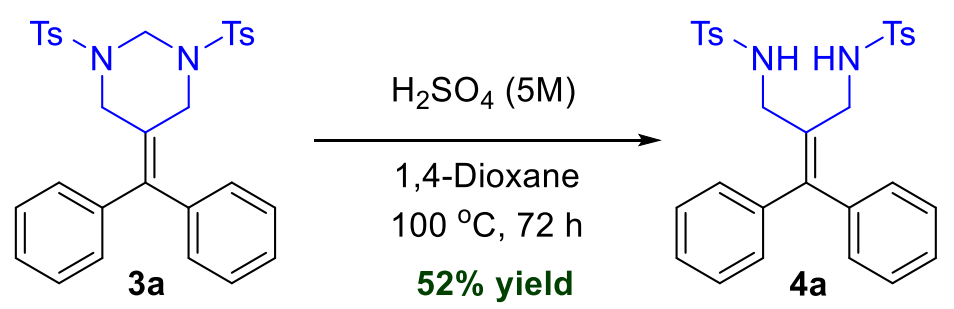

A solution of 3a $(0.1 \mathrm{mmol})$ was added into a mixture of $12 \mathrm{~mL}$ dioxane and $6 \mathrm{~mL}$ $5 \mathrm{M}$ sulfuric acid. The mixture was stirred for indicated time under gentle reflux till completion monitored by TLC. After cooling to room temperature, the mixture was 
evaporated to remove dioxane and the resulting mixture was extracted with ethyl acetate $(10 \mathrm{~mL} \times 3)$. The combined organic layer was dried over $\mathrm{Na}_{2} \mathrm{SO}_{4}$, filtered, and concentrated under decreased pressure. Then the solid was washed with small amount of methanol to afford pure product $4 \mathbf{a}$ as white solid ( $28 \mathrm{mg}, 52 \%$ yield, $\mathrm{mp}$ : 191.6-192. ${ }^{\circ} \mathrm{C}, \mathrm{R}_{f}=0.32$ (hexane/ethyl acetate 2:1). ${ }^{1} \mathbf{H} \mathbf{N M R}\left(500 \mathrm{MHz}, \mathrm{CDCl}_{3}\right) \delta$ $7.57(\mathrm{~d}, J=8.1 \mathrm{~Hz}, 4 \mathrm{H}), 7.25-7.18(\mathrm{~m}, 10 \mathrm{H}), 6.98-6.92(\mathrm{~m}, 4 \mathrm{H}), 5.12(\mathrm{t}, J=6.4 \mathrm{~Hz}$, 2H), $3.71(\mathrm{~d}, J=6.4 \mathrm{~Hz}, 4 \mathrm{H}), 2.40(\mathrm{~s}, 6 \mathrm{H}) ;{ }^{13} \mathbf{C}$ NMR $\left(125 \mathrm{MHz}, \mathrm{CDCl}_{3}\right) \delta 144.7$ (C), 143.4 (2 C), 140.4 (2 C), 136.4 (2 C), 129.6 (4 CH), 129.5 (C), 129.2 (4 CH), 128.2 (4 CH), $127.4(2 \mathrm{CH}), 127.1(4 \mathrm{CH}), 42.7\left(2 \mathrm{CH}_{2}\right), 21.5\left(2 \mathrm{CH}_{3}\right)$; HRMS (ESI) calcd. for $\mathrm{C}_{30} \mathrm{H}_{30} \mathrm{KN}_{2} \mathrm{O}_{4} \mathrm{~S}_{2}[\mathrm{M}+\mathrm{K}]: 585.1279$, found: 585.1278.

\section{Optimization of Catalytic Amidomethylative Tandem Reactions of $\alpha$ - Methylstyrene}

General procedure for optimization of the tandem reactions: An oven-dried Schlenk flask charged with bis(tosylamido)methane $2(0.35 \mathrm{mmol})$, catalyst $(10 \mathrm{~mol} \%)$ and a magnetic stir bar was evacuated and back-filled with nitrogen for three times. $\alpha$ Methylstyrene derivatives $5 \mathbf{a}(0.1 \mathrm{mmol})$ were added and followed by the addition of $2 \mathrm{~mL}$ DCE. The Schlenk tube was purged with nitrogen for $1 \mathrm{~min}$, then placed in an oil bath and stirred at certain conditions indicated in the table below. Then the reaction was stopped and the yield of product $\mathbf{6 a}$ was determined by ${ }^{1} \mathrm{H}$ NMR analysis with nitrobenzene as internal standard. 


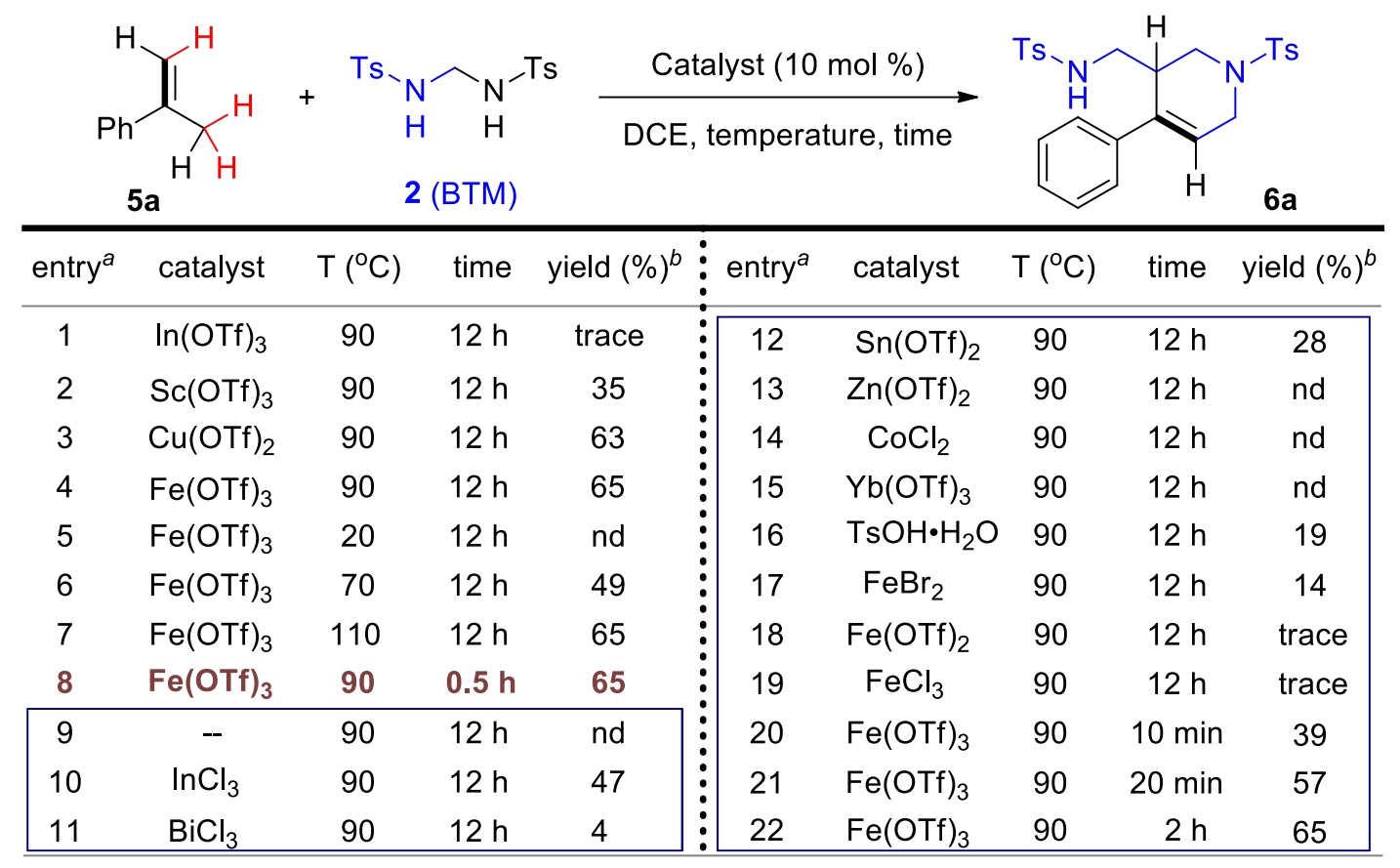

${ }^{a}$ Performed with 5a $(0.1 \mathrm{mmol}), 2(0.35 \mathrm{mmol})$, catalysts $(0.01 \mathrm{mmol})$ in $2.0 \mathrm{~mL}$ DCE. ${ }^{b 1} \mathrm{H}$ NMR yields.

\section{General Procedure for $\mathrm{Fe}(\mathrm{OTf})_{3}$-Catalyzed Catalytic Amidomethylative Tandem Reactions of $\alpha$-Methylstyrene (AMS) with BTM}<smiles>CC([17F])=CF</smiles>

5<smiles>[3H]NCN[AsH3-]</smiles>

2 (BTM)

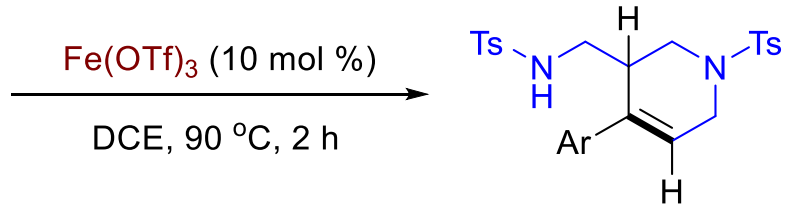

6

An oven-dried Schlenk flask charged with bis(tosylamido)methane (0.35 mmol), catalyst $\mathrm{Fe}(\mathrm{OTf})_{3}(10 \mathrm{~mol} \%)$ and a magnetic stir bar was evacuated and back-filled with nitrogen for three times. $\alpha$-Methylstyrene derivatives $(0.1 \mathrm{mmol})$ were added and followed by the addition of $2 \mathrm{~mL}$ DCE. The Schlenk tube was purged with nitrogen for $1 \mathrm{~min}$, then placed in an oil bath and stirred at $90{ }^{\circ} \mathrm{C}$ for $2 \mathrm{~h}$. After the reaction is done, the crude mixture was purified by flash chromatography (hexane/ethyl acetate $=6: 1-3: 1$ ). The fractions containing the product were collected and concentrated by vacuum to afford the compounds $\mathbf{6 a}-\mathbf{n}$. 


\section{Characterization of Products 6a-n}

\section{4-methyl-N-((4-phenyl-1-t osyl-1,2,3,6-tetrahydropyridin-3-yl)methyl)}

\section{benzenesulfonamide}<smiles>[As]NCC1CN([As])CC=C1c1ccccc1</smiles>

$6 a$

The title compound was prepared according to the general procedure as white solid (31.5 mg, 63\% yield, mp: $172.8-174.6{ }^{\circ} \mathrm{C}$ ). $\mathrm{R}_{f}=0.45$ (hexane/ethyl acetate $\left.2: 1\right) .{ }^{1} \mathbf{H}$ NMR $\left(300 \mathrm{MHz}, \mathrm{CDCl}_{3}\right) \delta=7.74(\mathrm{~d}, J=8.1 \mathrm{~Hz}, 2 \mathrm{H}), 7.65(\mathrm{~d}, J=8.1 \mathrm{~Hz}, 2 \mathrm{H}), 7.45-$ $7.31(\mathrm{~m}, 7 \mathrm{H}), 7.24(\mathrm{~d}, J=8.1 \mathrm{~Hz}, 2 \mathrm{H}), 5.99(\mathrm{br}, 1 \mathrm{H}), 5.15(\mathrm{t}, J=6.2 \mathrm{~Hz}, 1 \mathrm{H}), 4.17$ $\left(\mathrm{A}_{1} \mathrm{~B}_{1} \mathrm{X}_{1}, J_{\mathrm{AB}}=17.1 \mathrm{~Hz}, J_{\mathrm{AX}}=4.2 \mathrm{~Hz}, 1 \mathrm{H}\right), 4.09\left(\mathrm{~A}_{2} \mathrm{~B}_{2} \mathrm{X}_{2}, J_{\mathrm{AB}}=12.1 \mathrm{~Hz}, J_{\mathrm{AX}}=0\right.$ $\mathrm{Hz}, 1 \mathrm{H}), 3.38\left(\mathrm{~A}_{1} \mathrm{~B}_{1} \mathrm{X}_{1}, J_{\mathrm{BA}}=17.1 \mathrm{~Hz}, J_{\mathrm{BX}}=0 \mathrm{~Hz}, 1 \mathrm{H}\right), 3.23(\mathrm{br}, 1 \mathrm{H}), 3.00(\mathrm{t}, J=$ $6.6 \mathrm{~Hz}, 2 \mathrm{H}), 2.65\left(\mathrm{~A}_{2} \mathrm{~B}_{2} \mathrm{X}_{2}, J_{\mathrm{BA}}=12.1 \mathrm{~Hz}, J_{\mathrm{BX}}=2.7 \mathrm{~Hz}, 1 \mathrm{H}\right), 2.47(\mathrm{~s}, 3 \mathrm{H}), 2.40(\mathrm{~s}$, 3H); ${ }^{13} \mathrm{C}$ NMR (125 MHz, $\left.\mathrm{CDCl}_{3}\right) \delta=143.9$ (C), 143.4 (C), 138.0 (C), 137.0 (C), 136.7 (C), 133.3 (C), 129.9 (2 CH), 129.7 (2 CH), 128.8 (2 CH), $128.0(\mathrm{CH}), 127.5$ $(2 \mathrm{CH}), 127.1(2 \mathrm{CH}), 125.6(2 \mathrm{CH}), 121.1(\mathrm{CH}), 45.7\left(\mathrm{CH}_{2}\right), 44.5\left(\mathrm{CH}_{2}\right), 43.3\left(\mathrm{CH}_{2}\right)$, $38.0(\mathrm{CH}), 21.6\left(\mathrm{CH}_{3}\right), 21.5\left(\mathrm{CH}_{3}\right)$; HRMS (ESI) calcd. for $\mathrm{C}_{26} \mathrm{H}_{29} \mathrm{~N}_{2} \mathrm{O}_{4} \mathrm{~S}_{2}[\mathrm{M}+\mathrm{H}]$ : 497.1563, found: 497.1563.

\section{4-methyl-N-((4-m-tolyl-1-tosyl-1,2,3,6-tetrahydropyridin-3-}

yl)methyl)benzenesulfonamide<smiles>[3H]N1CC=C(c2cccc(C)c2)C(CNS)C1</smiles>

The title compound was prepared according to the general procedure as white solid (19.4 mg, 38\% yield, mp: $179.2-180.0{ }^{\circ} \mathrm{C}$ ). $\mathrm{R}_{f}=0.51$ (hexane/ethyl acetate $\left.2: 1\right) .{ }^{1} \mathbf{H}$ $\operatorname{NMR}\left(500 \mathrm{MHz}, \mathrm{CDCl}_{3}\right) \delta=7.71(\mathrm{~d}, J=8.2 \mathrm{~Hz}, 2 \mathrm{H}), 7.62(\mathrm{~d}, J=8.2 \mathrm{~Hz}, 2 \mathrm{H}), 7.36$ $(\mathrm{d}, J=8.2 \mathrm{~Hz}, 2 \mathrm{H}), 7.24-7.21(\mathrm{~m}, 3 \mathrm{H}), 7.19-7.15(\mathrm{~m}, 2 \mathrm{H}), 7.11(\mathrm{~d}, J=7.4 \mathrm{~Hz}, 1 \mathrm{H})$, 
$5.95\left(\mathrm{~A}_{1} \mathrm{~B}_{1} \mathrm{X}_{1}, J_{\mathrm{XA}}=4.4, J_{\mathrm{XB}}=1.2 \mathrm{~Hz}, 1 \mathrm{H}\right), 5.06(\mathrm{t}, J=6.4 \mathrm{~Hz}, 1 \mathrm{H}), 4.13\left(\mathrm{~A}_{1} \mathrm{~B}_{1} \mathrm{X}_{1}\right.$, $\left.J_{\mathrm{AB}}=17.2 \mathrm{~Hz}, J_{\mathrm{AX}}=4.4 \mathrm{~Hz}, 1 \mathrm{H}\right), 4.03\left(\mathrm{~A}_{2} \mathrm{~B}_{2} \mathrm{X}_{2}, J_{\mathrm{AB}}=12.0 \mathrm{~Hz}, J_{\mathrm{AX}}=0 \mathrm{~Hz}, 1 \mathrm{H}\right)$, $3.35\left(\mathrm{~A}_{1} \mathrm{~B}_{1} \mathrm{X}_{1}, J_{\mathrm{BA}}=17.2 \mathrm{~Hz}, J_{\mathrm{BX}}=1.2 \mathrm{~Hz}, 1 \mathrm{H}\right), 3.20(\mathrm{br}, 1 \mathrm{H}), 2.99-2.96(\mathrm{~m}, 2 \mathrm{H})$, $2.62\left(\mathrm{~A}_{2} \mathrm{~B}_{2} \mathrm{X}_{2}, J_{\mathrm{BA}}=12.0 \mathrm{~Hz}, J_{\mathrm{BX}}=3.0 \mathrm{~Hz}, 1 \mathrm{H}\right), 2.47(\mathrm{~s}, 3 \mathrm{H}), 2.41(\mathrm{~s}, 3 \mathrm{H}), 2.39(\mathrm{~s}$, 3H); ${ }^{13} \mathrm{C}$ NMR (125 MHz, $\left.\mathrm{CDCl}_{3}\right) \delta=143.9$ (C), 143.3 (C), 138.4 (C), 137.9 (C), $137.1(\mathrm{C}), 136.8(\mathrm{C}), 133.4(\mathrm{C}), 129.9(2 \mathrm{CH}), 129.7(2 \mathrm{CH}), 128.8(\mathrm{CH}), 128.7(\mathrm{CH})$, $127.5(2 \mathrm{CH}), 127.1(2 \mathrm{CH}), 126.4(\mathrm{CH}), 122.7(\mathrm{CH}), 120.8(\mathrm{CH}), 45.7\left(\mathrm{CH}_{2}\right), 44.5$ $\left(\mathrm{CH}_{2}\right), 43.3\left(\mathrm{CH}_{2}\right), 38.0(\mathrm{CH}), 21.6\left(\mathrm{CH}_{3}\right), 21.51\left(\mathrm{CH}_{3}\right), 21.50\left(\mathrm{CH}_{3}\right) ;$ HRMS (ESI) calcd. for $\mathrm{C}_{27} \mathrm{H}_{30} \mathrm{~N}_{2} \mathrm{NaO}_{4} \mathrm{~S}_{2}$ [M+Na]: 533.1539, found: 533.1539.

\section{N-((4-(biphenyl-4-yl)-1-tosyl-1,2,3,6-tetrahydropyridin-3-yl)methyl)-4- methylbenzenesulfonamide}<smiles>[As]NC[C@H]1CN([AsH])CC=C1c1ccc(-c2ccccc2)cc1</smiles>

The title compound was prepared according to the general procedure as white solid (32.8 mg, 47\% yield, mp: $207.8-208.6{ }^{\circ} \mathrm{C}$ ). $\mathrm{R}_{f}=0.48$ (hexane/ethyl acetate 2:1). ${ }^{1} \mathbf{H}$ NMR $\left(500 \mathrm{MHz}, \mathrm{CDCl}_{3}\right) \delta=7.74(\mathrm{~d}, J=7.9 \mathrm{~Hz}, 2 \mathrm{H}), 7.67(\mathrm{~d}, J=7.9 \mathrm{~Hz}, 2 \mathrm{H}), 7.63$ (t, $J=7.6 \mathrm{~Hz}, 4 \mathrm{H}), 7.49$ (t, $J=6.6 \mathrm{~Hz}, 4 \mathrm{H}), 7.40(\mathrm{~d}, J=7.9 \mathrm{~Hz}, 3 \mathrm{H}), 7.24$ (d, $J=7.9$ $\mathrm{Hz}, 2 \mathrm{H}), 6.06(\mathrm{br}, 1 \mathrm{H}), 5.09(\mathrm{t}, J=6.3 \mathrm{~Hz}, 1 \mathrm{H}), 4.21\left(\mathrm{~A}_{1} \mathrm{~B}_{1} \mathrm{X}_{1}, J_{\mathrm{AB}}=17.3 \mathrm{~Hz}, J_{\mathrm{AX}}=\right.$ $4.5 \mathrm{~Hz}, 1 \mathrm{H}), 4.09\left(\mathrm{~A}_{2} \mathrm{~B}_{2} \mathrm{X}_{2}, J_{\mathrm{AB}}=13.2 \mathrm{~Hz}, J_{\mathrm{AX}}=0 \mathrm{~Hz}, 1 \mathrm{H}\right), 3.42\left(\mathrm{~A}_{1} \mathrm{~B}_{1} \mathrm{X}_{1}, J_{\mathrm{BA}}=\right.$ $\left.17.3 \mathrm{~Hz}, J_{\text {BX }}=0 \mathrm{~Hz}, 1 \mathrm{H}\right), 3.30-3.25(\mathrm{~m}, 1 \mathrm{H}), 3.03-3.07(\mathrm{~m}, 2 \mathrm{H}), 2.67\left(\mathrm{~A}_{2} \mathrm{~B}_{2} \mathrm{X}_{2}, J_{\text {BA }}\right.$ $\left.=13.2, J_{\mathrm{BX}}=4.0 \mathrm{~Hz}, 1 \mathrm{H}\right), 2.48(\mathrm{~s}, 3 \mathrm{H}), 2.39(\mathrm{~s}, 3 \mathrm{H}) ;{ }^{13} \mathbf{C ~ N M R}\left(125 \mathrm{MHz}, \mathrm{CDCl}_{3}\right)$ $\delta=144.0$ (C), 143.4 (C), 140.8 (C), 140.4 (C), 136.8 (C), 136.7 (C), 136.5 (C), 133.4 (C), $129.9(2 \mathrm{CH}), 129.7(2 \mathrm{CH}), 128.9(2 \mathrm{CH}), 127.6(\mathrm{CH}), 127.5(2 \mathrm{CH}), 127.4(2$ $\mathrm{CH}), 127.0(2 \mathrm{CH}), 126.9(2 \mathrm{CH}), 125.9(2 \mathrm{CH}), 121.0(\mathrm{CH}), 45.8\left(\mathrm{CH}_{2}\right), 44.4\left(\mathrm{CH}_{2}\right)$, $43.3\left(\mathrm{CH}_{2}\right), 38.0(\mathrm{CH}), 21.6\left(\mathrm{CH}_{3}\right), 21.5\left(\mathrm{CH}_{3}\right)$; HRMS (ESI) calcd. for $\mathrm{C}_{32} \mathrm{H}_{33} \mathrm{~N}_{2} \mathrm{O}_{4} \mathrm{~S}_{2}[\mathrm{M}+\mathrm{H}]: 573.1876$, found: 573.1876 . 


\section{4-methyl-N-((4-(naphthalen-2-yl)-1-tosyl-1,2,3,6-tetrahydropyridin-3-}

yl)methyl)benzenesulfonamide<smiles>[13F]N1CC=C(c2ccc3ccccc3c2)C(C[AsH3-])C1</smiles>

The title compound was prepared according to the general procedure as white solid (27.9 mg, 51\% yield, mp: $162.0-164.0{ }^{\circ} \mathrm{C}$ ). $\mathrm{R}_{f}=0.47$ (hexane/ethyl acetate 2:1). ${ }^{1} \mathbf{H}$ $\operatorname{NMR}\left(500 \mathrm{MHz}, \mathrm{CDCl}_{3}\right) \delta=8.31(\mathrm{~d}, J=8.4 \mathrm{~Hz}, 1 \mathrm{H}), 7.84(\mathrm{~d}, J=8.1 \mathrm{~Hz}, 1 \mathrm{H}), 7.73$ $(\mathrm{d}, J=8.0 \mathrm{~Hz}, 3 \mathrm{H}), 7.62$ (t, $J=7.4 \mathrm{~Hz}, 1 \mathrm{H}), 7.58(\mathrm{~d}, J=8.0 \mathrm{~Hz}, 2 \mathrm{H}), 7.51(\mathrm{t}, J=7.4$ $\mathrm{Hz}, 1 \mathrm{H}), 7.39(\mathrm{~d}, J=8.0 \mathrm{~Hz}, 2 \mathrm{H}), 7.28(\mathrm{~s}, 1 \mathrm{H}), 7.22(\mathrm{~d}, J=8.0 \mathrm{~Hz}, 2 \mathrm{H}), 7.14(\mathrm{~d}, J=$ $8.4 \mathrm{~Hz}, 1 \mathrm{H}), 5.70(\mathrm{br}, 1 \mathrm{H}), 4.67(\mathrm{br}, 1 \mathrm{H}), 4.06\left(\mathrm{~A}_{1} \mathrm{~B}_{1} \mathrm{X}_{1}, J_{\mathrm{AB}}=17.0 \mathrm{~Hz}, J_{\mathrm{AX}}=4.0\right.$ $\mathrm{Hz}, 1 \mathrm{H}), 3.89\left(\mathrm{~A}_{2} \mathrm{~B}_{2} \mathrm{X}_{2}, J_{\mathrm{AB}}=12.2 \mathrm{~Hz}, J_{\mathrm{AX}}=4.5 \mathrm{~Hz}, 1 \mathrm{H}\right), 3.70(\mathrm{~d}, J=10.9 \mathrm{~Hz}, 1 \mathrm{H})$, 3.66-3.58 (m, 1H), $3.33\left(\mathrm{~A}_{1} \mathrm{~B}_{1} \mathrm{X}_{1}, J_{\mathrm{BA}}=17.0 \mathrm{~Hz}, J \mathrm{BX}=0 \mathrm{~Hz}, 1 \mathrm{H}\right), 2.72\left(\mathrm{~A}_{2} \mathrm{~B}_{2} \mathrm{X}_{2}, J\right.$ $\left.\mathrm{BA}=12.2 \mathrm{~Hz}, J_{\mathrm{BX}}=3.6 \mathrm{~Hz}, 1 \mathrm{H}\right), 2.67-2.62(\mathrm{~m}, 1 \mathrm{H}), 2.48(\mathrm{~s}, 3 \mathrm{H}), 2.42(\mathrm{~s}, 3 \mathrm{H}) ;{ }^{13} \mathrm{C}$ NMR $\left(125 \mathrm{MHz}, \mathrm{CDCl}_{3}\right) \delta=144.0$ (C), 143.2 (C), 139.5 (C), 138.8 (CH), 136.2 (C), 133.0 (C), 132.7 (C), $130.9(\mathrm{C}), 130.2$ (C), 129.9 (2 CH), 129.6 ( 2 CH), $128.5(\mathrm{CH})$, $128.4(\mathrm{CH}), 127.8(2 \mathrm{CH}), 127.2(2 \mathrm{CH}), 127.1(\mathrm{CH}), 126.0(\mathrm{CH}), 125.9(\mathrm{CH}), 123.5$ $(\mathrm{CH}), 122.6(\mathrm{CH}), 47.0\left(\mathrm{CH}_{2}\right), 46.0\left(\mathrm{CH}_{2}\right), 45.3\left(\mathrm{CH}_{2}\right), 38.4(\mathrm{CH}), 21.6\left(\mathrm{CH}_{3}\right), 21.5$ $\left(\mathrm{CH}_{3}\right)$; HRMS (ESI) calcd. for $\mathrm{C}_{30} \mathrm{H}_{30} \mathrm{KN}_{2} \mathrm{O}_{4} \mathrm{~S}_{2}[\mathrm{M}+\mathrm{K}]$ : 585.1278, found: 585.1277.

\section{N-((4-(4-fluorophenyl)-1-tosyl-1,2,3,6-tetrahydropyridin-3-yl)methyl)-4-} methylbenzenesulfonamide<smiles>[3H]NCC1CN([3H])CC=C1c1ccc(F)cc1</smiles> 
The title compound was prepared according to the general procedure as white solid (40.2 mg, $78 \%$ yield, mp: $171.2-172.0^{\circ} \mathrm{C}$ ). $\mathrm{R}_{f}=0.48$ (hexane/ethyl acetate $2: 1$ ). ${ }^{1} \mathbf{H}$ NMR $\left(500 \mathrm{MHz}, \mathrm{CDCl}_{3}\right) \delta=7.74(\mathrm{~d}, J=8.2 \mathrm{~Hz}, 2 \mathrm{H}), 7.64(\mathrm{~d}, J=8.2 \mathrm{~Hz}, 2 \mathrm{H}), 7.42-$ $7.33(\mathrm{~m}, 4 \mathrm{H}), 7.26(\mathrm{~d}, J=8.2 \mathrm{~Hz}, 2 \mathrm{H}), 7.06(\mathrm{t}, J=8.6 \mathrm{~Hz}, 2 \mathrm{H}), 5.93\left(\mathrm{~A}_{1} \mathrm{~B}_{1} \mathrm{X}_{1}, J_{\mathrm{XA}}=\right.$ $\left.4.3, J_{\mathrm{XB}}=1.2 \mathrm{~Hz}, 1 \mathrm{H}\right), 5.13(\mathrm{t}, J=6.3 \mathrm{~Hz}, 1 \mathrm{H}), 4.16\left(\mathrm{~A}_{1} \mathrm{~B}_{1} \mathrm{X}_{1}, J_{\mathrm{AB}}=17.2 \mathrm{~Hz}, J_{\mathrm{AX}}\right.$ $=4.3 \mathrm{~Hz}, 1 \mathrm{H}), 4.08\left(\mathrm{~A}_{2} \mathrm{~B}_{2} \mathrm{X}_{2}, J_{\mathrm{AB}}=14.8 \mathrm{~Hz}, J_{\mathrm{AX}}=0 \mathrm{~Hz}, 1 \mathrm{H}\right), 3.36\left(\mathrm{~A}_{1} \mathrm{~B}_{1} \mathrm{X}_{1}, J_{\mathrm{BA}}=\right.$ $\left.17.2 \mathrm{~Hz}, J_{\mathrm{BX}}=1.2 \mathrm{~Hz}, 1 \mathrm{H}\right), 3.19-3.17(\mathrm{~m}, 1 \mathrm{H}), 3.06-2.89(\mathrm{~m}, 2 \mathrm{H}), 2.64\left(\mathrm{~A}_{2} \mathrm{~B}_{2} \mathrm{X}_{2}, J\right.$ вА $\left.=14.8 \mathrm{~Hz}, J_{\mathrm{BX}}=2.0 \mathrm{~Hz}, 1 \mathrm{H}\right), 2.47(\mathrm{~s}, 3 \mathrm{H}), 2.41(\mathrm{~s}, 3 \mathrm{H}) ;{ }^{13} \mathrm{C} \mathbf{~ N M R}(125 \mathrm{MHz}$, $\left.\mathrm{CDCl}_{3}\right) \delta=162.6(\mathrm{~d}, J=246.2 \mathrm{~Hz})(\mathrm{C}-\mathrm{F}), 144.0(\mathrm{C}), 143.5(\mathrm{C}), 136.7$, (C) $136.1(\mathrm{C})$, $134.1(\mathrm{~d}, J=3.1 \mathrm{~Hz})(\mathrm{C}), 133.3(\mathrm{C}), 129.9(2 \mathrm{CH}), 129.7(2 \mathrm{CH}), 127.5(2 \mathrm{CH}), 127.3$ $(\mathrm{d}, J=8.0 \mathrm{~Hz})(2 \mathrm{CH}), 127.0(2 \mathrm{CH}), 121.1(\mathrm{CH}), 115.2(\mathrm{~d}, J=21.4 \mathrm{~Hz})(2 \mathrm{CH}), 45.6$ $\left(\mathrm{CH}_{2}\right), 44.4\left(\mathrm{CH}_{2}\right), 43.2\left(\mathrm{CH}_{2}\right), 38.3(\mathrm{CH}), 21.6\left(\mathrm{CH}_{3}\right), 21.5\left(\mathrm{CH}_{3}\right) ;{ }^{19} \mathbf{F}$ NMR (470 $\mathrm{MHz}, \mathrm{CDCl}_{3}$ ) $\delta-114.77$; HRMS (ESI) calcd. for $\mathrm{C}_{26} \mathrm{H}_{27} \mathrm{FKN}_{2} \mathrm{O}_{4} \mathrm{~S}_{2}[\mathrm{M}+\mathrm{K}]: 553.1028$, found: 553.1028 .

\section{N-((4-(4-chlorophenyl)-1-tosyl-1,2,3,6-tetrahydropyridin-3-yl)methyl)-4-}

\section{methylbenzenesulfonamide}<smiles>[NH3+]CC1CN([135I])CC=C1c1ccc(Cl)cc1</smiles>

The title compound was prepared according to the general procedure as white solid (38.0 mg, 72\% yield, mp: 199.2-200.0 ${ }^{\circ} \mathrm{C}$ ). $\mathrm{R}_{f}=0.47$ (hexane/ethyl acetate 2:1). ${ }^{\mathbf{1}} \mathbf{H}$ NMR $\left(500 \mathrm{MHz}, \mathrm{CDCl}_{3}\right) \delta=7.74(\mathrm{~d}, J=8.2 \mathrm{~Hz}, 2 \mathrm{H}), 7.65(\mathrm{~d}, J=8.2 \mathrm{~Hz}, 2 \mathrm{H}), 7.38$ $(\mathrm{d}, J=8.2 \mathrm{~Hz}, 2 \mathrm{H}), 7.33(\mathrm{~s}, 4 \mathrm{H}), 7.26(\mathrm{~d}, J=8.2 \mathrm{~Hz}, 2 \mathrm{H}), 5.98\left(\mathrm{~A}_{1} \mathrm{~B}_{1} \mathrm{X}_{1}, J_{\mathrm{XA}}=4.4\right.$, $\left.J_{\mathrm{XB}}=2.4 \mathrm{~Hz}, 1 \mathrm{H}\right), 5.20(\mathrm{t}, J=6.2 \mathrm{~Hz}, 1 \mathrm{H}), 4.16\left(\mathrm{~A}_{1} \mathrm{~B}_{1} \mathrm{X}_{1}, J_{\mathrm{AB}}=17.3 \mathrm{~Hz}, J_{\mathrm{AX}}=4.4\right.$ $\mathrm{Hz}, 1 \mathrm{H}), 4.09\left(\mathrm{~A}_{2} \mathrm{~B}_{2} \mathrm{X}_{2}, J_{\mathrm{AB}}=12.5 \mathrm{~Hz}, J_{\mathrm{AX}}=0 \mathrm{~Hz}, 1 \mathrm{H}\right), 3.36\left(\mathrm{~A}_{1} \mathrm{~B}_{1} \mathrm{X}_{1}, J_{\mathrm{BA}}=17.3\right.$ $\left.\mathrm{Hz}, J_{\mathrm{BX}}=2.4 \mathrm{~Hz}, 1 \mathrm{H}\right), 3.19-3.17(\mathrm{~m}, 1 \mathrm{H}), 3.04-2.90(\mathrm{~m}, 2 \mathrm{H}), 2.64\left(\mathrm{~A}_{2} \mathrm{~B}_{2} \mathrm{X}_{2}, J_{\mathrm{BA}}=\right.$ $\left.12.5, J_{\mathrm{BX}}=3.0 \mathrm{~Hz}, 1 \mathrm{H}\right), 2.47(\mathrm{~s}, 3 \mathrm{H}), 2.41(\mathrm{~s}, 3 \mathrm{H}) ;{ }^{13} \mathbf{C} \mathbf{N M R}\left(125 \mathrm{MHz}, \mathrm{CDCl}_{3}\right) \delta$ $=144.0(\mathrm{C}), 143.5(\mathrm{C}), 136.7$ (C), $136.4(\mathrm{C}), 136.0(\mathrm{C}), 133.9$ (C), $133.2(\mathrm{C}), 129.9$ 
(2 CH), $129.7(2 \mathrm{CH}), 128.9(2 \mathrm{CH}), 127.5(2 \mathrm{CH}), 127.0(2 \mathrm{CH}), 126.9(2 \mathrm{CH})$, $121.7(\mathrm{CH}), 45.7\left(\mathrm{CH}_{2}\right), 44.4\left(\mathrm{CH}_{2}\right), 43.1\left(\mathrm{CH}_{2}\right), 38.1(\mathrm{CH}), 21.6\left(\mathrm{CH}_{3}\right), 21.5\left(\mathrm{CH}_{3}\right)$; HRMS (ESI) calcd. for $\mathrm{C}_{26} \mathrm{H}_{27} \mathrm{ClKN}_{2} \mathrm{O}_{4} \mathrm{~S}_{2}$ [M+K]: 569.0732, found: 569.0733 .

\section{N-((4-(4-bromophenyl)-1-tosyl-1,2,3,6-tetrahydropyridin-3-yl)methyl)-4-}

\section{methylbenzenesulfonamide}<smiles>[NH3+]CC1CN([135I])CC=C1c1ccc(Br)cc1</smiles>

The title compound was prepared according to the general procedure as white solid (24.2 mg, 42\% yield, mp: 204.0-205.0 ${ }^{\circ} \mathrm{C}$ ). $\mathrm{R}_{f}=0.49$ (hexane/ethyl acetate 2:1). ${ }^{1} \mathbf{H}$ NMR $\left(500 \mathrm{MHz}, \mathrm{CDCl}_{3}\right) \delta 7.73(\mathrm{~d}, J=8.1 \mathrm{~Hz}, 2 \mathrm{H}), 7.64(\mathrm{~d}, J=8.1 \mathrm{~Hz}, 2 \mathrm{H}), 7.50$ (d, $J=8.2 \mathrm{~Hz}, 2 \mathrm{H}), 7.39$ (d, $J=8.2 \mathrm{~Hz}, 2 \mathrm{H}), 7.26$ (d, $J=7.2 \mathrm{~Hz}, 4 \mathrm{H}), 5.99$ (br, $1 \mathrm{H})$, $5.17(\mathrm{t}, J=6.2 \mathrm{~Hz}, 1 \mathrm{H}), 4.16\left(\mathrm{~A}_{1} \mathrm{~B}_{1} \mathrm{X}_{1}, J_{\mathrm{AB}}=17.2, J_{\mathrm{AX}}=4.2 \mathrm{~Hz}, 1 \mathrm{H}\right), 4.08\left(\mathrm{~A}_{2} \mathrm{~B}_{2} \mathrm{X}_{2}\right.$, $\left.J_{\mathrm{AB}}=12.2 \mathrm{~Hz}, J_{\mathrm{AX}}=0 \mathrm{~Hz}, 1 \mathrm{H}\right), 3.36\left(\mathrm{~A}_{1} \mathrm{~B}_{1} \mathrm{X}_{1}, J_{\mathrm{BA}}=17.2 \mathrm{~Hz}, J_{\mathrm{BX}}=1.2 \mathrm{~Hz}, 1 \mathrm{H}\right)$, 3.19-3.17 (m, 1H), 3.04-2.89 (m, 2H), $2.64\left(\mathrm{~A}_{2} \mathrm{~B}_{2} \mathrm{X}_{2}, J_{\mathrm{BA}}=12.2, J_{\mathrm{BX}}=2.3 \mathrm{~Hz}, 1 \mathrm{H}\right)$, 2.47 (s, 3H), 2.42 (s, 3H); ${ }^{13} \mathbf{C}$ NMR (125 MHz, $\left.\mathrm{CDCl}_{3}\right) \delta 144.1$ (C), $143.6(\mathrm{C}), 137.0$ (C), 136.8 (C), $136.2(\mathrm{C}), 133.4(\mathrm{C}), 132.0(2 \mathrm{CH}), 130.0(2 \mathrm{CH}), 129.8(2 \mathrm{CH}), 127.6$ $(2 \mathrm{CH}), 127.4(2 \mathrm{CH}), 127.1(2 \mathrm{CH}), 122.2(\mathrm{C}), 121.9(\mathrm{CH}), 45.8\left(\mathrm{CH}_{2}\right), 44.5\left(\mathrm{CH}_{2}\right)$, $43.3\left(\mathrm{CH}_{2}\right), 38.2(\mathrm{CH}), 21.7\left(\mathrm{CH}_{3}\right), 21.6\left(\mathrm{CH}_{3}\right)$; HRMS (ESI) calcd. for $\mathrm{C}_{26} \mathrm{H}_{27} \mathrm{BrKN}_{2} \mathrm{O}_{4} \mathrm{~S}_{2}[\mathrm{M}+\mathrm{K}]:$ 613.0227, found: 613.0220 .

\section{N-((4-(4-iodophenyl)-1-tosyl-1,2,3,6-tetrahydropyridin-3-yl)methyl)-4-} methylbenzenesulfonamide 


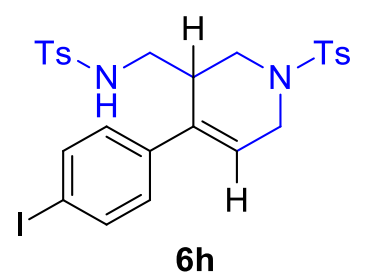

The title compound was prepared according to the general procedure as white solid (35.1 mg, 57\% yield, mp: $167.7-168.2^{\circ} \mathrm{C}$ ). $\mathrm{R}_{f}=0.53$ (hexane/ethyl acetate 2:1). ${ }^{1} \mathbf{H}$ NMR $\left(500 \mathrm{MHz}, \mathrm{CDCl}_{3}\right) \delta=7.74(\mathrm{~d}, J=8.2 \mathrm{~Hz}, 2 \mathrm{H}), 7.69(\mathrm{~d}, J=8.5 \mathrm{~Hz}, 2 \mathrm{H}), 7.64$ $(\mathrm{d}, J=8.2 \mathrm{~Hz}, 2 \mathrm{H}), 7.38(\mathrm{~d}, J=8.2 \mathrm{~Hz}, 2 \mathrm{H}), 7.26(\mathrm{~d}, J=8.2 \mathrm{~Hz}, 2 \mathrm{H}), 7.13(\mathrm{~d}, J=8.5$ $\mathrm{Hz}, 2 \mathrm{H}), 5.99$ (br, $1 \mathrm{H}), 5.14(\mathrm{t}, J=6.5 \mathrm{~Hz}, 1 \mathrm{H}), 4.15\left(\mathrm{~A}_{1} \mathrm{~B}_{1} \mathrm{X}_{1}, J_{\mathrm{AB}}=17.4, J_{\mathrm{AX}}=4.4\right.$ $\mathrm{Hz}, 1 \mathrm{H}), 4.10\left(\mathrm{~A}_{2} \mathrm{~B}_{2} \mathrm{X}_{2}, J_{\mathrm{AB}}=12.2 \mathrm{~Hz}, J_{\mathrm{AX}}=0 \mathrm{~Hz}, 1 \mathrm{H}\right), 3.36\left(\mathrm{~A}_{1} \mathrm{~B}_{1} \mathrm{X}_{1}, J_{\mathrm{BA}}=17.4\right.$, $\left.J_{\mathrm{BX}}=1.2 \mathrm{~Hz}, 1 \mathrm{H}\right), 3.19-3.17(\mathrm{~m}, 1 \mathrm{H}), 2.99-2.93(\mathrm{~m}, 2 \mathrm{H}), 2.63\left(\mathrm{~A}_{2} \mathrm{~B}_{2} \mathrm{X}_{2}, J_{\mathrm{BA}}=12.2\right.$, $\left.J_{\mathrm{BX}}=2.5 \mathrm{~Hz}, 1 \mathrm{H}\right), 2.47(\mathrm{~s}, 3 \mathrm{H}), 2.42(\mathrm{~s}, 3 \mathrm{H}) ;{ }^{13} \mathbf{C ~ N M R}\left(125 \mathrm{MHz}, \mathrm{CDCl}_{3}\right) \delta 144.0$ (C) , 143.5(C), 137.8 (2 CH) , 137.5 (C), 136.6 (C), 136.2 (C), 133.3 (C), $129.9(2$ $\mathrm{CH}), 129.7(2 \mathrm{CH}), 127.5(2 \mathrm{CH}), 127.4(2 \mathrm{CH}), 127.0(2 \mathrm{CH}), 121.8(\mathrm{CH}), 93.6(\mathrm{C})$, $45.7\left(\mathrm{CH}_{2}\right), 44.4\left(\mathrm{CH}_{2}\right), 43.2\left(\mathrm{CH}_{2}\right), 38.0(\mathrm{CH}), 21.6\left(\mathrm{CH}_{3}\right), 21.5\left(\mathrm{CH}_{3}\right) ;$ HRMS (ESI) calcd. for $\mathrm{C}_{26} \mathrm{H}_{27} \mathrm{IKN}_{2} \mathrm{O}_{4} \mathrm{~S}_{2}$ [M+K]: 661.0088, found: 661.0089 .

\section{N-((4-(3-chlorophenyl)-1-tosyl-1,2,3,6-tetrahydropyridin-3-yl)methyl)-4- methylbenzenesulfonamide}<smiles>[Y5]NC[C@H]1CN([135I])CC=C1c1cccc(Cl)c1</smiles>

$6 \mathbf{i}$

The title compound was prepared according to the general procedure as white solid (24.7 mg, 47\% yield, mp: 197.0-199.0 ${ }^{\circ} \mathrm{C}$ ). $\mathrm{R}_{f}=0.45$ (hexane/ethyl acetate 2:1). ${ }^{1} \mathbf{H}$ NMR $\left(500 \mathrm{MHz}, \mathrm{CDCl}_{3}\right) \delta 7.71(\mathrm{~d}, J=8.2 \mathrm{~Hz}, 2 \mathrm{H}), 7.65(\mathrm{~d}, J=8.2 \mathrm{~Hz}, 2 \mathrm{H}), 7.37$ $(\mathrm{d}, J=8.2 \mathrm{~Hz}, 3 \mathrm{H}), 7.28-7.24(\mathrm{~m}, 5 \mathrm{H}), 5.99\left(\mathrm{~A}_{1} \mathrm{~B}_{1} \mathrm{X}_{1}, J_{\mathrm{XA}}=4.4, J_{\mathrm{XB}}=1.2 \mathrm{~Hz}, 1 \mathrm{H}\right)$, $5.16(\mathrm{t}, J=6.2 \mathrm{~Hz}, 1 \mathrm{H}), 4.16\left(\mathrm{~A}_{1} \mathrm{~B}_{1} \mathrm{X}_{1}, J_{\mathrm{AB}}=17.3, J_{\mathrm{AX}}=4.4 \mathrm{~Hz}, 1 \mathrm{H}\right), 4.07\left(\mathrm{~A}_{2} \mathrm{~B}_{2} \mathrm{X}_{2}\right.$, $\left.J_{\mathrm{AB}}=12.2 \mathrm{~Hz}, J_{\mathrm{AX}}=0 \mathrm{~Hz}, 1 \mathrm{H}\right), 3.35\left(\mathrm{~A}_{1} \mathrm{~B}_{1} \mathrm{X}_{1}, J_{\mathrm{BA}}=17.3 \mathrm{~Hz}, J_{\mathrm{BX}}=1.2,1 \mathrm{H}\right), 3.19-$ 
$3.13(\mathrm{~m}, 1 \mathrm{H}), 3.03-2.86(\mathrm{~m}, 2 \mathrm{H}), 2.60\left(\mathrm{~A}_{2} \mathrm{~B}_{2} \mathrm{X}_{2}, J_{\mathrm{BA}}=12.2, J_{\mathrm{BX}}=2.9 \mathrm{~Hz}, 1 \mathrm{H}\right), 2.45$ (s, 3H), 2.39 (s, 3H); ${ }^{13} \mathrm{C}$ NMR (125 MHz, $\mathrm{CDCl}_{3}$ ) $\delta 144.0$ (C), 143.5 (C), $139.9(\mathrm{C})$, 136.6(C), $136.0(\mathrm{C}), 134.8(\mathrm{C}), 133.2(\mathrm{C}), 130.0(\mathrm{CH}), 129.9$ (2 CH), $129.7(2 \mathrm{CH})$, $128.0(\mathrm{CH}), 127.5(2 \mathrm{CH}), 127.1(2 \mathrm{CH}), 125.9(\mathrm{CH}), 123.7(\mathrm{CH}), 122.4(\mathrm{CH}), 45.6$ $\left(\mathrm{CH}_{2}\right), 44.3\left(\mathrm{CH}_{2}\right), 43.1\left(\mathrm{CH}_{2}\right), 38.0(\mathrm{CH}), 21.6\left(\mathrm{CH}_{3}\right), 21.5\left(\mathrm{CH}_{3}\right) ;$ HRMS (ESI) calcd. for $\mathrm{C}_{26} \mathrm{H}_{28} \mathrm{ClN}_{2} \mathrm{O}_{4} \mathrm{~S}_{2}[\mathrm{M}+\mathrm{H}]$ ] 531.1174 , found: 531.1168 .

\section{N-((4-(3-bromophenyl)-1-tosyl-1,2,3,6-tetrahydropyridin-3-yl)methyl)-4-}

\section{methylbenzenesulfonamide}

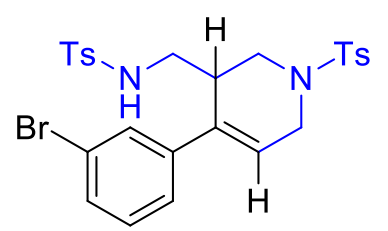

6j

The title compound was prepared according to the general procedure as white solid (23.3 mg, 41\% yield, mp: $208.2-209.0{ }^{\circ} \mathrm{C}$ ). $\mathrm{R}_{f}=0.52$ (hexane/ethyl acetate $2: 1$ ). ${ }^{1} \mathbf{H}$ NMR $\left(500 \mathrm{MHz}, \mathrm{CDCl}_{3}\right) \delta=7.74(\mathrm{~d}, J=8.1 \mathrm{~Hz}, 2 \mathrm{H}), 7.68(\mathrm{~d}, J=8.2 \mathrm{~Hz}, 2 \mathrm{H})$, $7.54(\mathrm{~s}, 1 \mathrm{H}), 7.45(\mathrm{~d}, J=7.9 \mathrm{~Hz}, 1 \mathrm{H}), 7.39(\mathrm{~d}, J=8.1 \mathrm{~Hz}, 2 \mathrm{H}), 7.34(\mathrm{~d}, J=7.9 \mathrm{~Hz}$, $1 \mathrm{H}), 7.28-7.23(\mathrm{~m}, 3 \mathrm{H}), 6.01\left(\mathrm{~A}_{1} \mathrm{~B}_{1} \mathrm{X}_{1}, J_{\mathrm{XA}}=4.4, J_{\mathrm{XB}}=1.2 \mathrm{~Hz}, 1 \mathrm{H}\right), 5.20(\mathrm{t}, J=$ $6.2 \mathrm{~Hz}, 1 \mathrm{H}), 4.18\left(\mathrm{~A}_{1} \mathrm{~B}_{1} \mathrm{X}_{1}, J_{\mathrm{AB}}=17.5, J_{\mathrm{AX}}=4.4 \mathrm{~Hz}, 1 \mathrm{H}\right), 4.10\left(\mathrm{~A}_{2} \mathrm{~B}_{2} \mathrm{X}_{2}, J_{\mathrm{AB}}=\right.$ $\left.12.0, J_{\mathrm{AX}}=0 \mathrm{~Hz}, 1 \mathrm{H}\right), 3.37\left(\mathrm{~A}_{1} \mathrm{~B}_{1} \mathrm{X}_{1}, J_{\mathrm{BA}}=17.5, J_{\mathrm{BX}}=1.2 \mathrm{~Hz}, 1 \mathrm{H}\right), 3.19-3.17(\mathrm{~m}$, $1 \mathrm{H}), 3.03-2.89(\mathrm{~m}, 2 \mathrm{H}), 2.62\left(\mathrm{~A}_{2} \mathrm{~B}_{2} \mathrm{X}_{2}, J_{\mathrm{BA}}=12.0, J_{\mathrm{BX}}=2.0 \mathrm{~Hz}, 1 \mathrm{H}\right), 2.47(\mathrm{~s}, 3 \mathrm{H})$, 2.41 (s, 3H); ${ }^{13} \mathrm{C}$ NMR $\left(125 \mathrm{MHz}, \mathrm{CDCl}_{3}\right) \delta=144.0(\mathrm{C}), 143.5(\mathrm{C}), 140.2(\mathrm{C})$, 136.6 (C), $135.9(\mathrm{C}), 133.3(\mathrm{C}), 131.0(\mathrm{CH}), 130.3(\mathrm{CH}), 129.9$ (2 CH), $129.7(2$ $\mathrm{CH}), 128.8(\mathrm{CH}), 127.5(2 \mathrm{CH}), 127.1(2 \mathrm{CH}), 124.2(\mathrm{CH}), 123.0(\mathrm{C}), 122.4(\mathrm{CH})$, $45.7\left(\mathrm{CH}_{2}\right), 44.4\left(\mathrm{CH}_{2}\right), 43.1\left(\mathrm{CH}_{2}\right), 38.0(\mathrm{CH}), 21.6\left(\mathrm{CH}_{3}\right), 21.5\left(\mathrm{CH}_{3}\right) ;$ HRMS (ESI) calcd. for $\mathrm{C}_{26} \mathrm{H}_{27} \mathrm{BrKN}_{2} \mathrm{O}_{4} \mathrm{~S}_{2}$ [M+K]: 613.0227, found: 613.0227.

\section{N-((4-(3-iodophenyl)-1-tosyl-1,2,3,6-tetrahydropyridin-3-yl)methyl)-4- methylbenzenesulfonamide}




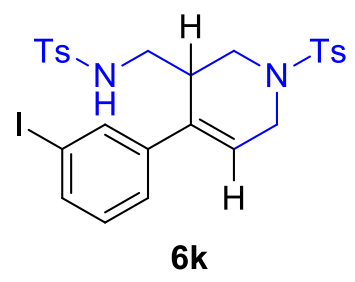

The title compound was prepared according to the general procedure as white solid (25.9 mg, $42 \%$ yield, mp: $215.0-216.7^{\circ} \mathrm{C}$ ). $\mathrm{R}_{f}=0.47$ (hexane/ethyl acetate 2:1). ${ }^{1} \mathbf{H}$ NMR $\left(500 \mathrm{MHz}, \mathrm{CDCl}_{3}\right) \delta$ 7.75-7.69 (m, 3H), 7.67-7.63 (m, 3H), $7.36(\mathrm{t}, J=8.0$ $\mathrm{Hz}, 3 \mathrm{H}), 7.27-7.24(\mathrm{~m}, 2 \mathrm{H}), 7.09(\mathrm{t}, J=8.0 \mathrm{~Hz}, 1 \mathrm{H}), 5.97\left(\mathrm{~A}_{1} \mathrm{~B}_{1} \mathrm{X}_{1}, J\right.$ ХА $=4.2, J$ ХВ $=1.2 \mathrm{~Hz}, 1 \mathrm{H}), 5.09(\mathrm{t}, J=6.0 \mathrm{~Hz}, 1 \mathrm{H}), 4.15\left(\mathrm{~A}_{1} \mathrm{~B}_{1} \mathrm{X}_{1}, J_{\mathrm{AB}}=17.3, J_{\mathrm{AX}}=4.2 \mathrm{~Hz}, 1 \mathrm{H}\right)$, $4.06\left(\mathrm{~A}_{2} \mathrm{~B}_{2} \mathrm{X}_{2}, J_{\mathrm{AB}}=12.2 \mathrm{~Hz}, J_{\mathrm{AX}}=0 \mathrm{~Hz}, 1 \mathrm{H}\right), 3.34\left(\mathrm{~A}_{1} \mathrm{~B}_{1} \mathrm{X}_{1}, J_{\mathrm{BA}}=17.3 \mathrm{~Hz}, J_{\mathrm{BX}}=\right.$ $1.2 \mathrm{~Hz}, 1 \mathrm{H}), 3.15-3.13(\mathrm{~m}, 1 \mathrm{H}), 3.00-2.89(\mathrm{~m}, 2 \mathrm{H}), 2.60\left(\mathrm{~A}_{2} \mathrm{~B}_{2} \mathrm{X}_{2}, J_{\text {BA }}=12.2, J_{\text {BX }}\right.$ $=2.5 \mathrm{~Hz}, 1 \mathrm{H}), 2.45(\mathrm{~s}, 3 \mathrm{H}), 2.40(\mathrm{~s}, 3 \mathrm{H}) ;{ }^{13} \mathrm{C}$ NMR $\left(125 \mathrm{MHz}, \mathrm{CDCl}_{3}\right) \delta 144.0(\mathrm{C})$, 143.5 (C), 140.3 (C), 137.0 (CH), $136.6(\mathrm{C}), 135.8$ (C), 134.7 (CH), $133.4(\mathrm{C}), 130.5$ $(\mathrm{CH}), 130.0(2 \mathrm{CH}), 129.8(2 \mathrm{CH}), 127.5(2 \mathrm{CH}), 127.1(2 \mathrm{CH}), 124.8(\mathrm{CH}), 122.4$ $(\mathrm{CH}), 95.0(\mathrm{C}), 45.7\left(\mathrm{CH}_{2}\right), 44.4\left(\mathrm{CH}_{2}\right), 43.1\left(\mathrm{CH}_{2}\right), 38.0(\mathrm{CH}), 21.6\left(\mathrm{CH}_{3}\right), 21.5$ $\left(\mathrm{CH}_{3}\right)$; HRMS (ESI) calcd. for $\mathrm{C}_{26} \mathrm{H}_{28} \mathrm{IN}_{2} \mathrm{O}_{4} \mathrm{~S}_{2}[\mathrm{M}+\mathrm{H}]:$ 623.0530, found: 623.0530 .

\section{4-methyl-N-((1-tosyl-4-(4-(trifluoromethyl)phenyl)-1,2,3,6-tetrahydropyridin-}

\section{3-yl)methyl)benzenesulfonamide}<smiles>[3H]NCC1CN([3H])CC=C1c1ccc(C(F)(F)F)cc1</smiles>

The title compound was prepared according to the general procedure as white solid (17.7 mg, 32\% yield, mp: 88.5-90.5 ${ }^{\circ} \mathrm{C}$ ). $\mathrm{R}_{f}=0.34$ (hexane/ethyl acetate $2: 1$ ). ${ }^{1} \mathbf{H}$ NMR $\left(500 \mathrm{MHz}, \mathrm{CDCl}_{3}\right) \delta=7.74(\mathrm{~d}, J=8.2 \mathrm{~Hz}, 2 \mathrm{H}), 7.67-7.60(\mathrm{~m}, 4 \mathrm{H}), 7.51(\mathrm{~d}, J$ $=8.2 \mathrm{~Hz}, 2 \mathrm{H}), 7.39(\mathrm{~d}, J=8.1 \mathrm{~Hz}, 2 \mathrm{H}), 7.24(\mathrm{~d}, J=8.1 \mathrm{~Hz}, 2 \mathrm{H}), 6.08\left(\mathrm{~A}_{1} \mathrm{~B}_{1} \mathrm{X}_{1}, J \mathrm{XA}\right.$ $\left.=4.2, J_{\mathrm{XB}}=1.2 \mathrm{~Hz}, 1 \mathrm{H}\right), 5.14(\mathrm{t}, J=6.0 \mathrm{~Hz}, 1 \mathrm{H}), 4.22\left(\mathrm{~A}_{1} \mathrm{~B}_{1} \mathrm{X}_{1}, J_{\mathrm{AB}}=17.2 \mathrm{~Hz}, J_{\mathrm{AX}}\right.$ $=4.2 \mathrm{~Hz}, 1 \mathrm{H}), 4.12\left(\mathrm{~A}_{2} \mathrm{~B}_{2} \mathrm{X}_{2}, J_{\mathrm{AB}}=12.5 \mathrm{~Hz}, J_{\mathrm{AX}}=0 \mathrm{~Hz}, 1 \mathrm{H}\right), 3.39\left(\mathrm{~A}_{1} \mathrm{~B}_{1} \mathrm{X}_{1}, J_{\mathrm{BA}}=\right.$ 
$\left.17.2 \mathrm{~Hz}, J_{\text {вх }}=1.2 \mathrm{~Hz}, 1 \mathrm{H}\right), 3.27-3.24(\mathrm{~m}, 1 \mathrm{H}), 3.04-2.99(\mathrm{~m}, 1 \mathrm{H}), 2.95-2.86(\mathrm{~m}$, $1 \mathrm{H}), 2.66\left(\mathrm{~A}_{2} \mathrm{~B}_{2} \mathrm{X}_{2}, J_{\mathrm{BA}}=12.5 \mathrm{~Hz}, J_{\mathrm{BX}}=2.5 \mathrm{~Hz}, 1 \mathrm{H}\right), 2.48(\mathrm{~s}, 3 \mathrm{H}), 2.41(\mathrm{~s}, 3 \mathrm{H}) ;{ }^{13} \mathrm{C}$ NMR $\left(125 \mathrm{MHz}, \mathrm{CDCl}_{3}\right) \delta=144.1$ (C), 143.6 (C), 141.6 (C), 136.6 (C), 136.1 (C), $133.3(\mathrm{C}), 130.0(\mathrm{q}, J=32.1 \mathrm{~Hz})(\mathrm{C}), 129.9(2 \mathrm{CH}), 129.7(2 \mathrm{CH}), 127.5(2 \mathrm{CH})$, $127.0(2 \mathrm{CH}), 126.0(2 \mathrm{CH}), 125.7(\mathrm{q}, J=4.1 \mathrm{~Hz})(2 \mathrm{CH}), 124.0(\mathrm{q}, J=270.4 \mathrm{~Hz})$

$\left(\mathrm{CF}_{3}\right), 123.3(\mathrm{CH}), 45.7\left(\mathrm{CH}_{2}\right), 44.4\left(\mathrm{CH}_{2}\right), 43.1\left(\mathrm{CH}_{2}\right), 38.2(\mathrm{CH}), 21.6\left(\mathrm{CH}_{3}\right), 21.5$ $\left(\mathrm{CH}_{3}\right) ;{ }^{19} \mathbf{F}$ NMR $\left(470 \mathrm{MHz}, \mathrm{CDCl}_{3}\right) \delta-63.28$; HRMS (ESI) calcd. for $\mathrm{C}_{27} \mathrm{H}_{27} \mathrm{~F}_{3} \mathrm{~N}_{2} \mathrm{NaO}_{4} \mathrm{~S}_{2}$ [M+Na]: 587.1256, found: 587.1256.

\section{4-methyl-N-((1-tosyl-4-(3-(trifluoromethyl)phenyl)-1,2,3,6-tetrahydropyridin-}

\section{3-yl)methyl)benzenesulfonamide}<smiles>[13CH3]N1CC=C(c2cccc(C(F)(F)F)c2)C(C[NH3+])C1</smiles>

The title compound was prepared according to the general procedure as white solid (11.9 mg, $21 \%$ yield, mp: $\left.187.2-188.0{ }^{\circ} \mathrm{C}\right) .{ }^{1} \mathbf{H} \mathbf{~ N M R}\left(500 \mathrm{MHz}, \mathrm{CDCl}_{3}\right) \delta=7.74(\mathrm{~d}$, $J=8.0 \mathrm{~Hz}, 2 \mathrm{H}), 7.61(\mathrm{~m}, 5 \mathrm{H}), 7.52(\mathrm{~d}, J=7.5 \mathrm{~Hz}, 2 \mathrm{H}), 7.39(\mathrm{~d}, J=8.0 \mathrm{~Hz}, 2 \mathrm{H}), 7.25$ $(\mathrm{d}, J=8.0 \mathrm{~Hz}, 2 \mathrm{H}), 6.07(\mathrm{br}, 1 \mathrm{H}), 5.12(\mathrm{t}, J=6.3 \mathrm{~Hz}, 1 \mathrm{H}), 4.22\left(\mathrm{~A}_{1} \mathrm{~B}_{1} \mathrm{X}_{1}, J_{\mathrm{AB}}=17.5\right.$, $\left.J_{\mathrm{AX}}=4.1 \mathrm{~Hz}, 1 \mathrm{H}\right), 4.12\left(\mathrm{~A}_{2} \mathrm{~B}_{2} \mathrm{X}_{2}, J_{\mathrm{AB}}=12.5, J_{\mathrm{BX}}=0 \mathrm{~Hz}\right), 3.39\left(\mathrm{~A}_{1} \mathrm{~B}_{1} \mathrm{X}_{1}, J_{\mathrm{BA}}=\right.$ $\left.17.5, J_{\mathrm{BX}}=0 \mathrm{~Hz}, 1 \mathrm{H}\right), 3.25-3.23(\mathrm{~m}, 1 \mathrm{H}), 3.04-2.92(\mathrm{~m}, 2 \mathrm{H}), 2.65\left(\mathrm{~A}_{2} \mathrm{~B}_{2} \mathrm{X}_{2}, J_{\mathrm{BA}}=\right.$ $\left.12.5, J_{\mathrm{BX}}=0 \mathrm{~Hz}, 1 \mathrm{H}\right), 2.48(\mathrm{~s}, 3 \mathrm{H}), 2.41(\mathrm{~s}, 3 \mathrm{H}) ;{ }^{13} \mathbf{C ~ N M R}\left(125 \mathrm{MHz}, \mathrm{CDCl}_{3}\right) \delta=$ 144.0 (C), 143.5 (C), 138.8 (C), 136.6 (C), 136.0 (C) 133.4 (C), 129.9 (2 CH), 129.7 ( $2 \mathrm{CH}), 129.4(\mathrm{q}, J=31.8 \mathrm{~Hz}$ ) (C), $129.3(\mathrm{CH}), 128.8(\mathrm{CH}), 127.4(2 \mathrm{CH}), 127.0$ $(2 \mathrm{CH}), 124.8(\mathrm{q}, J=270.8 \mathrm{~Hz})\left(\mathrm{CF}_{3}\right), 124.71(\mathrm{q}, J=3.4 \mathrm{~Hz})(\mathrm{CH}), 122.9(\mathrm{CH})$, $122.45(\mathrm{q}, J=3.6 \mathrm{~Hz})(\mathrm{CH}), 45.6\left(\mathrm{CH}_{2}\right), 44.3\left(\mathrm{CH}_{2}\right), 43.1\left(\mathrm{CH}_{2}\right), 38.1(\mathrm{CH}), 21.6$ $\left(\mathrm{CH}_{3}\right), 21.5\left(\mathrm{CH}_{3}\right)$;; ${ }^{19} \mathbf{F}$ NMR $\left(470 \mathrm{MHz}, \mathrm{CDCl}_{3}\right) \delta-62.63 ;$; HRMS (ESI) calcd. for $\mathrm{C}_{27} \mathrm{H}_{27} \mathrm{~F}_{3} \mathrm{KN}_{2} \mathrm{O}_{4} \mathrm{~S}_{2}$ [M+K]: 603.0996, found: 603.0996.

\section{4-methyl-N-((2-tosyl-2,3,9,9a-tetrahydro-1H-indeno[2,1-c]pyridin-9a- yl)methyl)benzenesulfonamide}




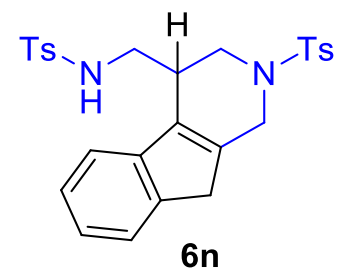

The title compound was prepared according to the general procedure as light yellow solid (8.9 mg, $17 \%$ yield, mp: $195.5-197.0^{\circ} \mathrm{C}$ ). $\mathrm{R}_{f}=0.43$ (hexane/ethyl acetate $2: 1$ ). ${ }^{1} \mathbf{H}$ NMR $\left(500 \mathrm{MHz}, \mathrm{CDCl}_{3}\right) \delta 7.77(\mathrm{~d}, J=8.2 \mathrm{~Hz}, 2 \mathrm{H}), 7.72(\mathrm{~d}, J=8.2 \mathrm{~Hz}, 2 \mathrm{H})$, 7.44-7.27 (m, 7H), 7.18 (t, $J=7.3 \mathrm{~Hz}, 1 \mathrm{H}), 5.18$ (t, $J=6.5 \mathrm{~Hz}, 1 \mathrm{H}), 4.45$ (d, $J=16.4$ $\mathrm{Hz}, 1 \mathrm{H}), 4.07\left(\mathrm{ABX}, J_{\mathrm{AB}}=12.3 \mathrm{~Hz}, J_{\mathrm{AX}}=0 \mathrm{~Hz}, 1 \mathrm{H}\right), 3.57(\mathrm{~d}, J=16.4 \mathrm{~Hz}, 1 \mathrm{H})$, 3.38-3.29 (m, 1H), 3.26-3.24 (m, 2H), 3.16-3.14 (m, 1H), 3.10-3.04 (m, 1H), 2.64 $\left(\mathrm{ABX}, J_{\mathrm{BA}}=12.3 \mathrm{~Hz}, J_{\mathrm{BX}}=2.6 \mathrm{~Hz}, 1 \mathrm{H}\right), 2.45(\mathrm{~s}, 3 \mathrm{H}), 2.40(\mathrm{~s}, 3 \mathrm{H}) ;{ }^{13} \mathbf{C}$ NMR $(125$ $\left.\mathrm{MHz}, \mathrm{CDCl}_{3}\right) \delta 144.1$ (C), 143.6 (C), 143.0 (C), 142.4 (C), 137.4 (C), 137.3 (C), $136.1(\mathrm{C}), 134.0(\mathrm{C}), 130.1(2 \mathrm{CH}), 129.9(2 \mathrm{CH}), 127.5(2 \mathrm{CH}), 127.1(2 \mathrm{CH}), 126.9$ $(\mathrm{CH}), 125.2(\mathrm{CH}), 124.0(\mathrm{CH}), 118.7(\mathrm{CH}), 46.4\left(\mathrm{CH}_{2}\right), 44.8\left(\mathrm{CH}_{2}\right), 43.3\left(\mathrm{CH}_{2}\right), 38.6$ $(\mathrm{CH}), 35.5\left(\mathrm{CH}_{2}\right), 21.7\left(\mathrm{CH}_{3}\right), 21.6\left(\mathrm{CH}_{3}\right)$; HRMS (ESI) calcd. for $\mathrm{C}_{27} \mathrm{H}_{28} \mathrm{KN}_{2} \mathrm{O}_{4} \mathrm{~S}_{2}$ [M+K]: 547.1122, found: 547.1127.

\section{Large Scale (gram) Synthesis of Representative Product 6a}

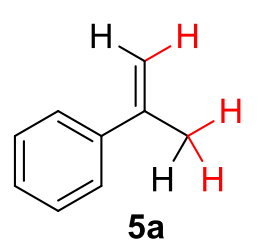

$2 \mathrm{mmol}$ scale

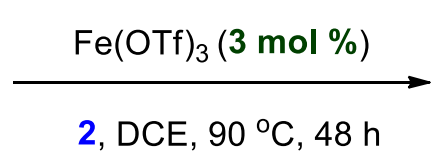

0.55 gram $6 a, 56 \%$ yield<smiles>[As]NCC1CN([As])CC=C1c1ccccc1</smiles>

$6 a$

The reaction was conducted according to the general procedure with minor modification: to an oven-dried $75 \mathrm{~mL}$ Schlenk tube, which was charged with a magnetic stir bar, bis(tosylamido)methane (7 mmol), catalyst $\mathrm{Fe}(\mathrm{OTf})_{3}(3 \mathrm{~mol} \%)$ were added, then evacuated and back filled with nitrogen for three times. The Teflon screw cap was replaced with a rubber septum. $\alpha$-Methylstyrene $(2 \mathrm{mmol})$ was added and followed by $30 \mathrm{~mL}$ DCE. The Schlenk tube was then purged with nitrogen for 1 min and the rubber septum was replaced with a Teflon screw cap. The reaction 
mixture was then stirred at $90{ }^{\circ} \mathrm{C}$ for $48 \mathrm{~h}$. After the reaction is done, the reaction mixture was purified by flash chromatography (hexane/ethyl acetate $=6: 1-3: 1$ ). The fractions containing the product were collected and concentrated by rotary evaporation to afford the compound $\mathbf{6 a}(0.55 \mathrm{~g}, 56 \%$ yield $)$.

\section{Mechanistic Study for $\mathrm{Fe}(\mathrm{OTf})_{3}$-Catalyzed Amidomethylative Tandem Reactions of $\alpha$-Phenylstyrene with BTM}

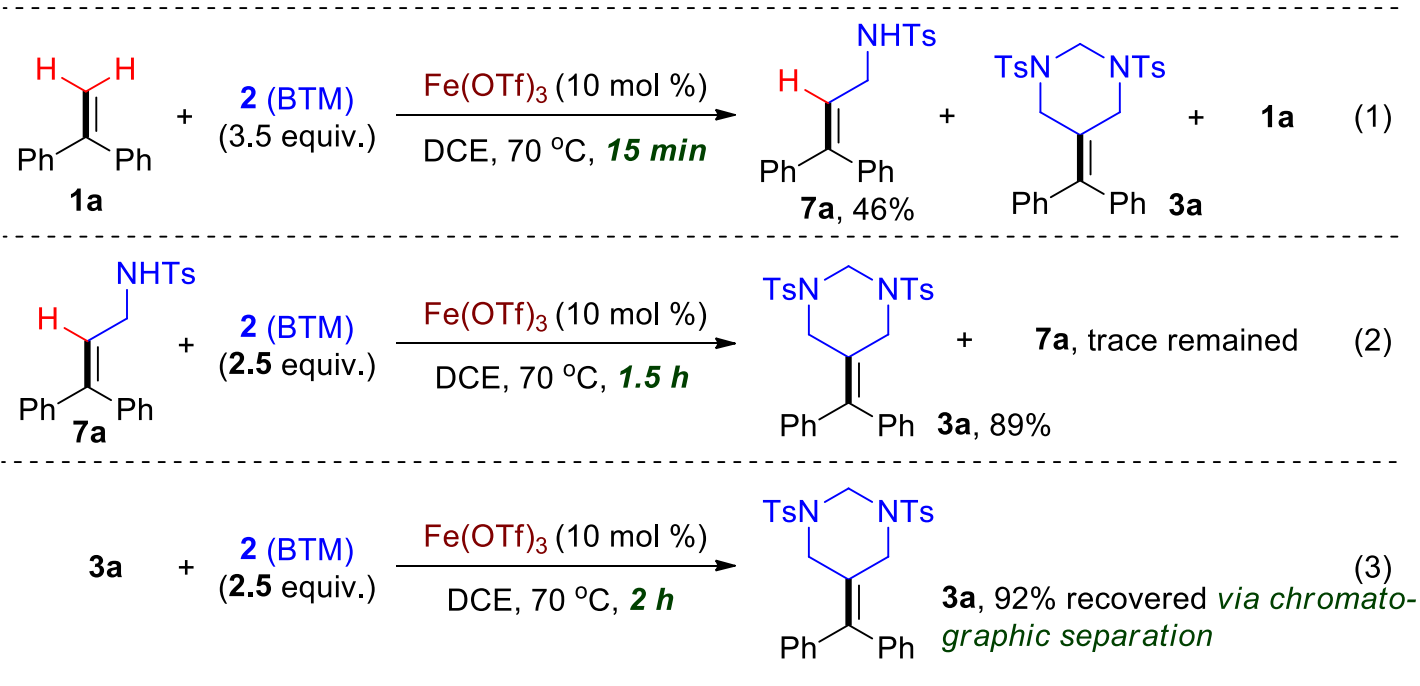

According to the general procedure: to an oven-dried Schlenk tube charged with a magnetic stir bar, bis(tosylamido)methane $(0.35 \mathrm{mmol})$, catalyst Fe(OTf $)_{3}(10 \mathrm{~mol} \%)$ was added stepwise. The Schlenk tube was then evacuated and back filled with nitrogen for three times. $\alpha$-Phenylstyrene $1 \mathbf{a}(0.1 \mathrm{mmol})$ was added and followed by $2 \mathrm{~mL}$ DCE . The resultant solution was then purged with nitrogen for $1 \mathrm{~min}$ and the rubber septum was replaced with a Teflon screw cap, which was elevated to $70{ }^{\circ} \mathrm{C}$ and reacted for $15 \mathrm{~min}$. Then the reaction was stopped and purified by flash chromatography (hexane/ethyl acetate $=6: 1-3: 1$ ), in order to capture the intermediate of this tandem reactions, the fractions containing the product were collected and concentrated by vacuum, affording the expected product $7 \mathbf{a}$ in $46 \%$ yield. (eq. 1)

The following reaction showed in equation $\mathbf{2}$ was performed to verify the mechanism we proposed in the text. To an oven-dried Schlenk tube charged with bis(tosylamido)methane $(31.2 \mathrm{mg}, 0.088 \mathrm{mmol})$, catalyst $\mathrm{Fe}(\mathrm{OTf})_{3}(10 \mathrm{~mol} \%)$ and a 
magnetic stir bar, which was dried via standard Schlenk technic, the above-mentioned 7a $(12.8 \mathrm{mg}, 0.0352 \mathrm{mmol})$ was added and followed by $1 \mathrm{~mL}$ DCE. The resultant solution was then purged with nitrogen for 1 min and stirred at $70{ }^{\circ} \mathrm{C}$ for $1.5 \mathrm{~h}$, through TLC, we exactly observed a nearly full conversion of the intermediate 7a, led to formation of molecule 3a in $89 \%$ isolated yield. (Silica-column with eluent of hexane/ethyl acetate $=6: 1-3: 1)$.

A subsequent experiment was reasonably directed to verify the terminate step of our

reactions (eq. 3). To an oven-dried Schlenk tube, which was charged with bis(tosylamido)methane $(17.7 \mathrm{mg}, 0.05 \mathrm{mmol})$, catalyst $\mathrm{Fe}(\mathrm{OTf})_{3}(10 \mathrm{~mol} \%)$ and a magnetic stir bar. 3a obtained from the afore-mentioned reaction (11.2 mg, 0.02 mmol) was added and followed by $0.4 \mathrm{~mL} \mathrm{DCE}$. The reaction system was then purged with nitrogen for $1 \mathrm{~min}$ and stirred at $70{ }^{\circ} \mathrm{C}$ for $2 \mathrm{~h}$, the product $3 \mathbf{a}$ was recovered in quantitative yield after the purification by flash chromatography (hexane/ethyl acetate $=6: 1-3: 1)$.

\section{Spectra of Intermediate $7 \mathbf{a}$}

\section{N-(3,3-diphenylallyl)-4-methylbenzenesulfonamide}

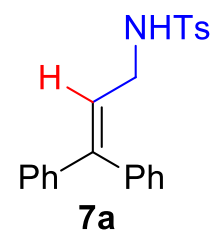

The title compound was prepared according to the general procedure as white solid (17.0 mg, 50\% yield, mp: $\left.127.5-129.5^{\circ} \mathrm{C}\right) . \mathrm{R}_{f}=0.47$ (hexane/ethyl acetate 2:1). ${ }^{1} \mathbf{H}$ NMR $\left(500 \mathrm{MHz}, \mathrm{CDCl}_{3}\right) \delta 7.69(\mathrm{~d}, J=8.2 \mathrm{~Hz}, 2 \mathrm{H}), 7.32-7.28(\mathrm{~m}, 3 \mathrm{H}), 7.26-7.22$ (m, 5H), 7.08-7.02 (m, 2H), $5.91(\mathrm{t}, J=7.0 \mathrm{~Hz}, 1 \mathrm{H}), 4.60(\mathrm{t}, J=5.9 \mathrm{~Hz}, 1 \mathrm{H}), 3.66$ (m, 2H), $2.40(\mathrm{~s}, 3 \mathrm{H}) ;{ }^{13} \mathrm{C}$ NMR (125 MHz, $\left.\mathrm{CDCl}_{3}\right) \delta 145.5$ (C), $143.5(\mathrm{C}), 141.3$ (C), $138.5(\mathrm{C}), 137.1(\mathrm{C}), 129.8(2 \mathrm{CH}), 129.6(2 \mathrm{CH}), 128.5(2 \mathrm{CH}), 128.2(2 \mathrm{CH})$, $127.8(\mathrm{CH}), 127.7(\mathrm{CH}), 127.5(2 \mathrm{CH}), 127.2(2 \mathrm{CH}), 123.3(\mathrm{CH}), 42.5\left(\mathrm{CH}_{2}\right), 21.6$ $\left(\mathrm{CH}_{3}\right)$; HRMS (SI) calcd. for $\mathrm{C}_{22} \mathrm{H}_{21} \mathrm{KNO}_{2} \mathrm{~S}[\mathrm{M}+\mathrm{K}]$ : 402.0925 , found: 402.0927 . 


\section{A Potential Alternative Pathway to the Amidomethylative Tandem Reactions of $\alpha$-Phenylstyrene with BTM}

While 3a was proven to hydrolyze and gave 1,3-diamide $\mathbf{4 a}$, it is interesting to note that treating $\mathbf{4 a}$ under the catalytic conditions resulted in the formation of $\mathbf{3 a}$ in $83 \%$ yield, as shown in the following reaction:

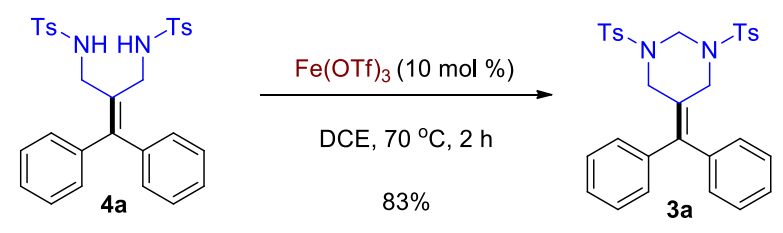

Based on all the current observations, the following potential alternative pathway for the formation of 3a, which is based on a hypothesized iterative intermolecular amidomethylation reaction, cannot currently been experimentally excluded. Considering the n-nucleophiles (nitrogen atom) tend to be more nucleophilic than $\pi$ nucleophiles (the alkene), which is in accord with the aza-Prins cyclization reactions of unsaturated tosylamides known to date (see references $2 \mathrm{a}$ and 7 in the manuscript), we prefer to adopt the current mechanism for understanding the process, while do not exclude the following alternative.

A hypothesized iterative intermolecular amidomethylation pathway:

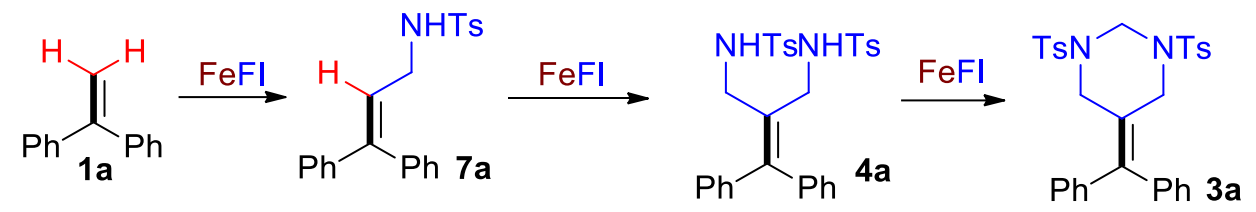

\section{Mechanistic Study on Reaction Intermediates by a HRMS Experiment}

To a $10 \mathrm{~mL}$ of Schlenk tube equipped with a magnetic stirring bar, substrate $\alpha$-BTM (3.5 equiv.) and $\mathrm{Fe}(\mathrm{OTf})_{3}(5 \mathrm{~mol} \%)$ were added stepwise under air. The mixture was then evacuated and backfilled with nitrogen for three times. $\alpha$-Methylstyrene $(0.025$ mmol) and $0.5 \mathrm{~mL}$ DCE were added subsequently. After stirring at $90{ }^{\circ} \mathrm{C}$ for $0.5 \mathrm{~h}$, the reaction mixture was cooled to room temperature. Then the mixture was diluted 
with $2.5 \mathrm{~mL}$ DCE, from which $50 \mu \mathrm{L}$ mixture was taken and added to a vial with 1 mL DCE, followed by immediate injection into HRMS. While 6a was detected as a major species, the appearance of signal at 509.1555 (Calcd. for 509.1563) matched the $\mathrm{m} / \mathrm{z}$ of the intermediate $\mathbf{K}$ or $\mathbf{L}$. The low intensity of the presumably unstable species $\mathbf{K}$ or $\mathbf{L}$ is consistent with the rationalized tandem termination mechanism.

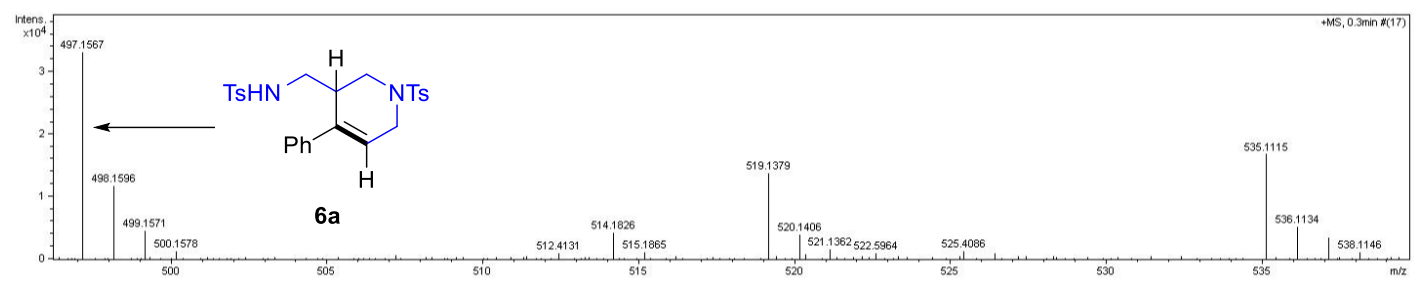

Figure S1 High Resolution Mass Spectrometry of the reaction mixture (showing $\mathrm{m} / \mathrm{z}$ of $\mathbf{6 a}$ )
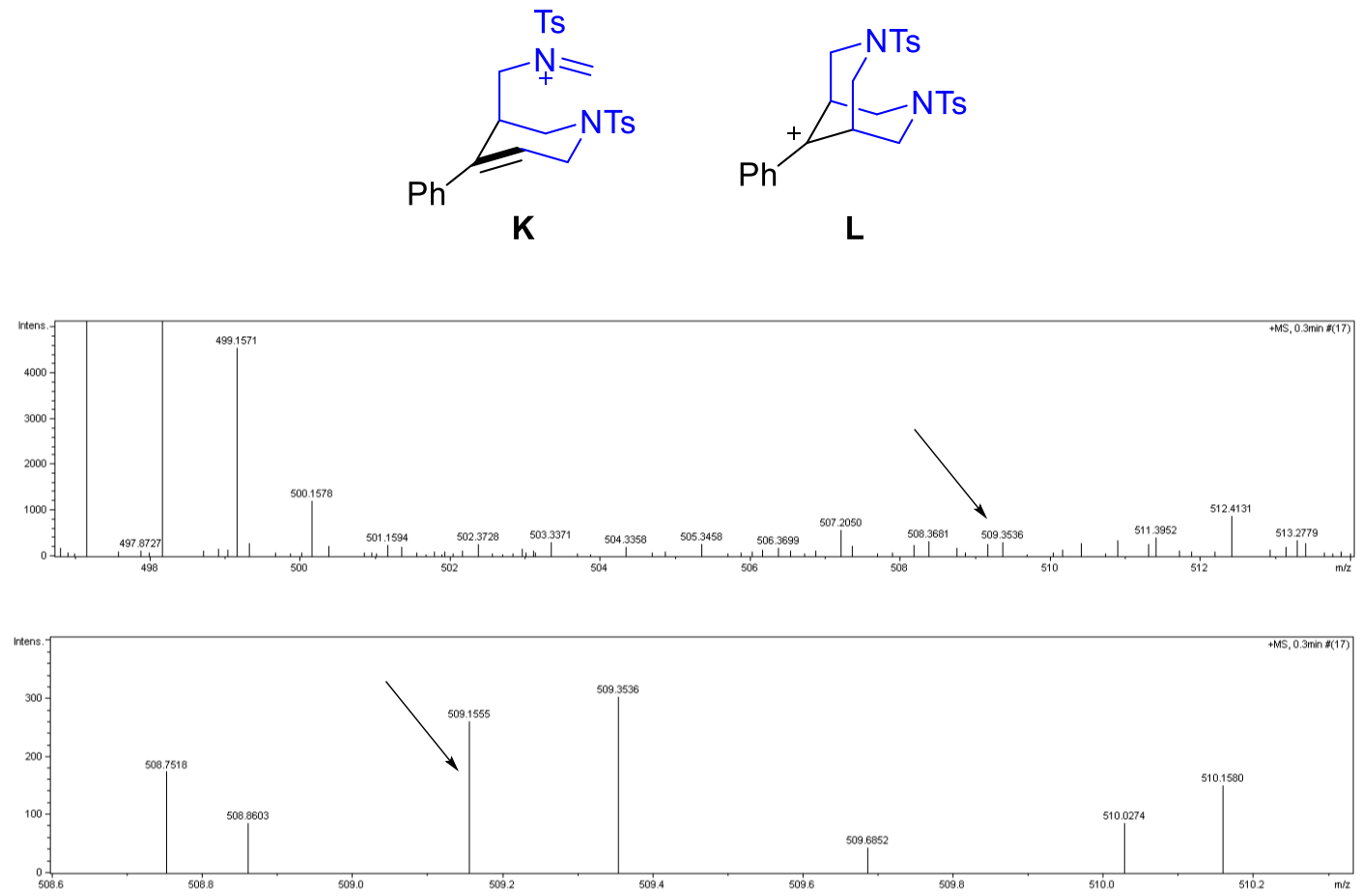

Figure S2 High Resolution Mass Spectrometry of the reaction mixture (showing $\mathrm{m} / \mathrm{z}$ that matches $\mathbf{K}$ or $\mathbf{L}$ ) 
15. Mechanistic Study for $\mathrm{Fe}(\mathrm{OTf})_{3}$-Catalyzed Amidomethylative Tandem Reactions of $\alpha$-Methylstyrene with BTM

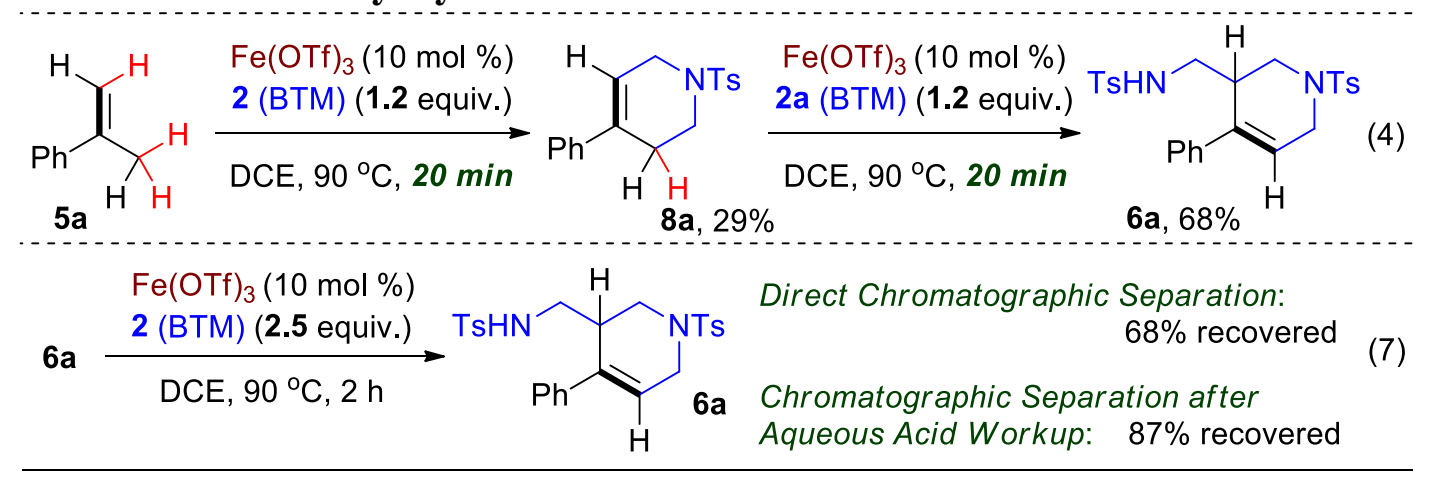

To look for reaction intermediates, experiments were performed with modified procedures. $\alpha$-Methylstyrene $\mathbf{5 a}(0.1 \mathrm{mmol})$ was treated by 1.2 equivalents of BTM in the presence of $10 \mathrm{~mol} \% \mathrm{Fe}(\mathrm{OTf})_{3}$ and stopped after $20 \mathrm{~min}$. 8a was isolated in $29 \%$ yield from the reaction mixture. Repetition of this reaction was carried to accumulate enough 8a for further experiments.

8a was then subjected to the reaction with 1.2 equivalents of BTM and $10 \mathrm{~mol} \%$ $\mathrm{Fe}(\mathrm{OTf})_{3}$. After $20 \mathrm{~min}$, the reaction was monitored by TLC and the desired product 6a was observed then isolated in $68 \%$ yield.

A subsequent experiment was reasonably directed to verify the terminate step of our reactions. To an oven-dried Schlenk tube, which was charged with bis(tosylamido)methane $(17.7 \mathrm{mg}, 0.05 \mathrm{mmol})$, catalyst $\mathrm{Fe}(\mathrm{OTf})_{3}(10 \mathrm{~mol} \%)$ and a magnetic stir bar. 6a obtained from the afore-mentioned reaction $(9.9 \mathrm{mg}, 0.02 \mathrm{mmol})$ was added and followed by $0.4 \mathrm{~mL}$ DCE. The reaction system was then purged with nitrogen for $1 \mathrm{~min}$ and stirred at $90{ }^{\circ} \mathrm{C}$ for $2 \mathrm{~h}$, the product $6 \mathbf{a}$ was recovered in $68 \%$ yield after direct chromatographic separation. Moreover, the product 6a was obtained in $87 \%$ isolated yield with flash chromatography after work-up with acid (hexane/ethyl acetate $=6: 1-3: 1)$. 


\section{Spectra of Intermediate 8 a}

\section{4-phenyl-1-tosyl-1,2,3,6-tetrahydropyridine}<smiles>[NH3+]CC=Cc1ccccc1</smiles>

The title compound was prepared according to the general procedure as white solid (16.9 mg, 27\% yield). $\mathrm{R}_{f}=0.74$ (hexane/ethyl acetate 2:1). ${ }^{1} \mathbf{H}$ NMR (500 MHz, $\left.\mathrm{CDCl}_{3}\right) \delta 7.71(\mathrm{~d}, J=8.0 \mathrm{~Hz}, 2 \mathrm{H}), 7.33(\mathrm{~m}, 7 \mathrm{H}), 5.95(\mathrm{br}, 1 \mathrm{H}), 3.76(\mathrm{~d}, J=2.4 \mathrm{~Hz}$, 2H), $3.32(\mathrm{t}, J=5.7 \mathrm{~Hz}, 2 \mathrm{H}), 2.61(\mathrm{br}, 2 \mathrm{H}), 2.43(\mathrm{~s}, 3 \mathrm{H}) ;{ }^{13} \mathbf{C ~ N M R}\left(125 \mathrm{MHz}, \mathrm{CDCl}_{3}\right)$ $\delta 143.7(\mathrm{C}), 140.2(\mathrm{C}), 135.4(\mathrm{C}), 133.2(\mathrm{C}), 129.7$ (2 CH), 128.5 (2 CH), 127.8 (2 $\mathrm{CH}), 127.6(\mathrm{CH}), 125.0(2 \mathrm{CH}), 119.0(\mathrm{CH}), 45.3\left(\mathrm{CH}_{2}\right), 43.1\left(\mathrm{CH}_{2}\right), 27.6\left(\mathrm{CH}_{2}\right)$, $21.6\left(\mathrm{CH}_{3}\right)$; HRMS (ESI) calcd. for $\mathrm{C}_{18} \mathrm{H}_{19} \mathrm{KNO}_{2} \mathrm{~S}[\mathrm{M}+\mathrm{K}]$ : 352.0774, found: 352.0774 .

\section{Deuterium labeling Experiments}

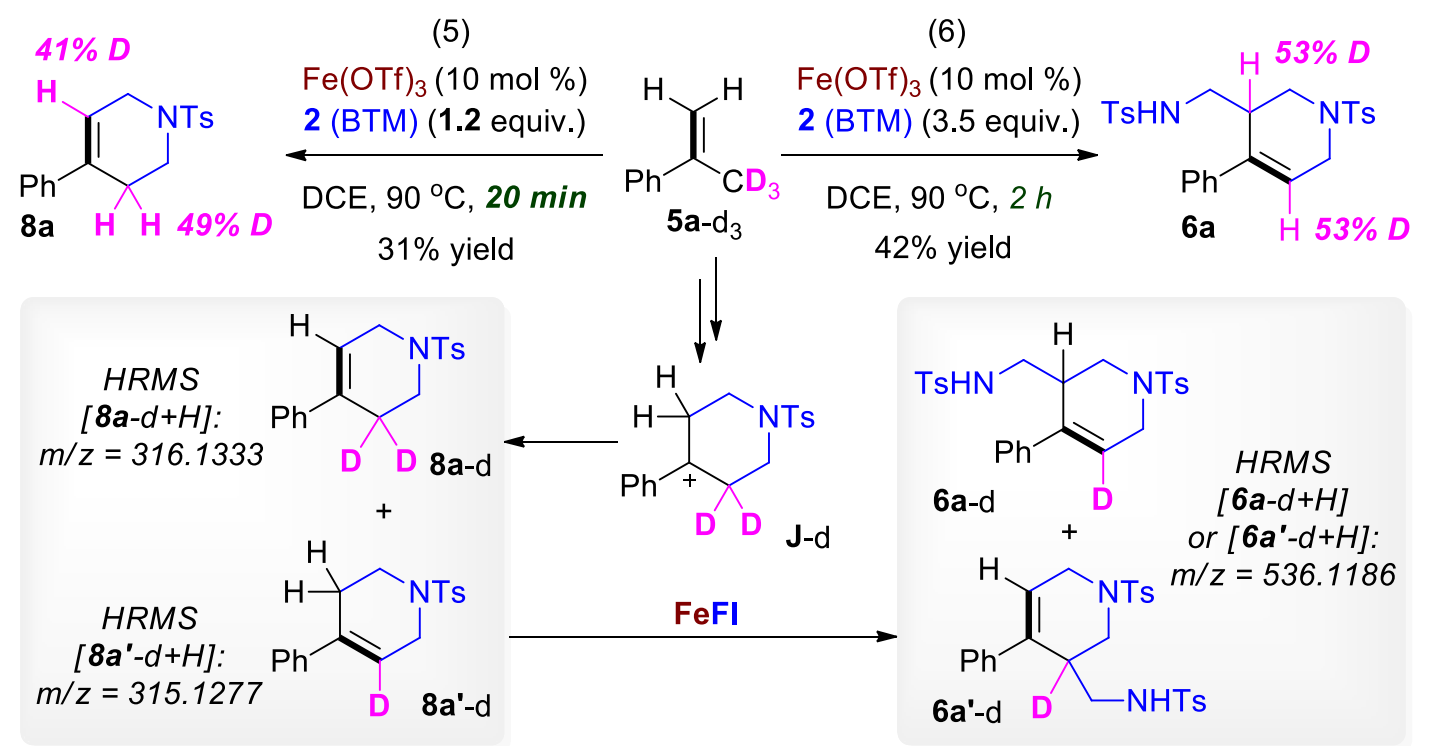

Deuterium labelling experiments were performed for further mechanistic details. $\alpha$ Methyl-d $\mathrm{d}_{3}$-styrene $\left(\mathbf{5 a}-\mathrm{d}_{3}\right)(12.1 \mathrm{~g}, 0.1 \mathrm{mmol})$ was treated with BTM under the standard catalytic conditions. One reaction was quenched after $20 \mathrm{~min}$ (eq. 5) and 8a was isolated as white solid (9.7mg, 31\% yield). ${ }^{1} \mathbf{H} \mathbf{~ N M R}\left(500 \mathrm{MHz}, \mathrm{CDCl}_{3}\right) \delta 7.72$ 
(d, $J=8.1 \mathrm{~Hz}, 2 \mathrm{H}), 7.33(\mathrm{~d}, J=8.1 \mathrm{~Hz}, 2 \mathrm{H}), 7.31-7.25(\mathrm{~m}, 5 \mathrm{H}), 5.97-5.93(\mathrm{~m}$, 0.59H), 3.76 (br, 2H), 3.36-3.27 (m, 2H), 2.63-2.58 (m, 1.02H), 2.43 (s, 3H). HRMS experiment of this sample identified two species: HRMS (ESI) calcd. for $\mathrm{C}_{18} \mathrm{H}_{19} \mathrm{DNO}_{2} \mathrm{~S}[\mathrm{M}+\mathrm{H}]: 315.1272$, found: 315.1277. This species matches the proposed 8a'-d. HRMS (ESI) calcd. for $\mathrm{C}_{18} \mathrm{H}_{18} \mathrm{D}_{2} \mathrm{NO}_{2} \mathrm{~S}$ [M+H]: 316.1335, found: 316.1333. This species matches the proposed 8a-d.

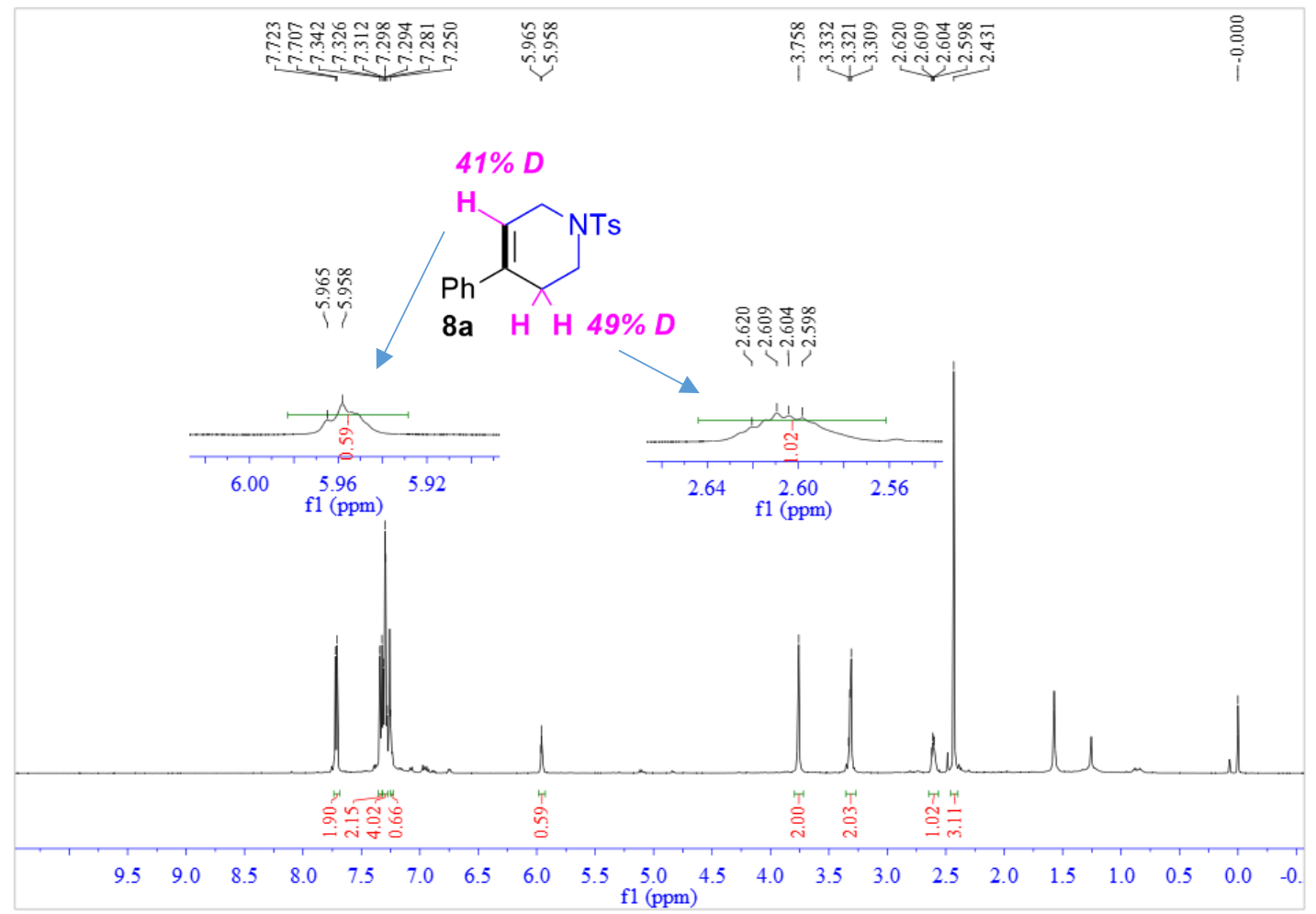

HRMS of 8a from equation 5:

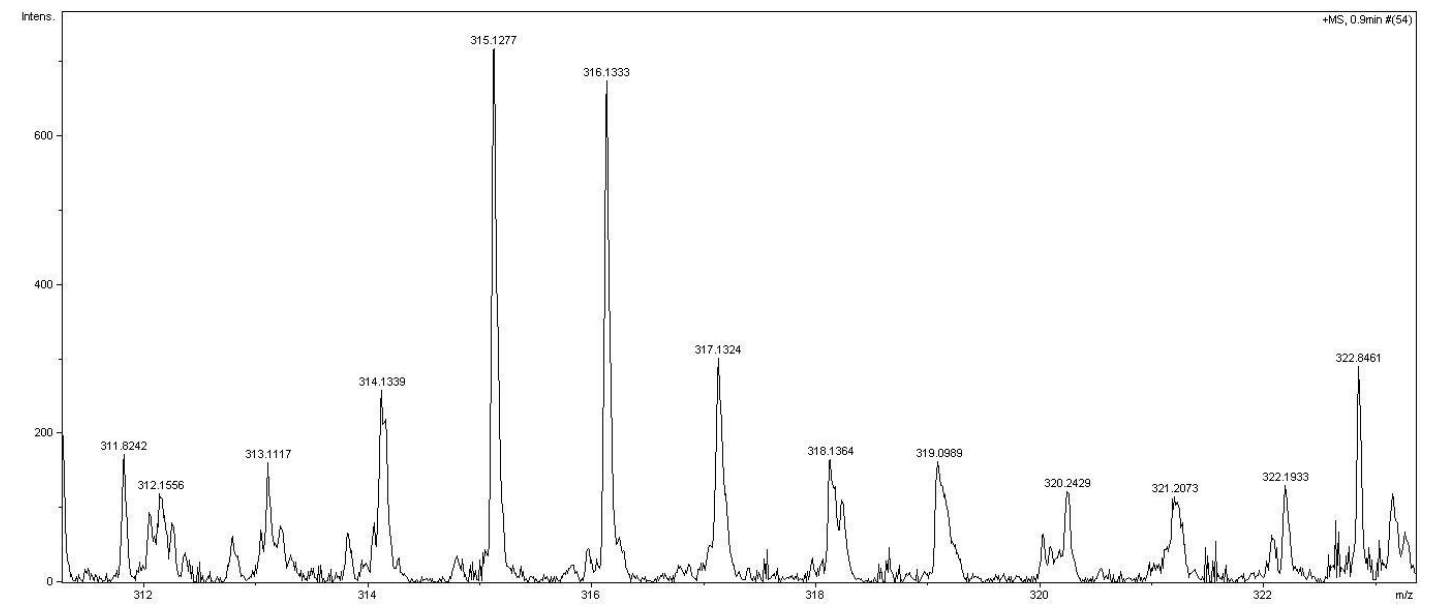




\section{Simulated HRMS of 8a'-d}

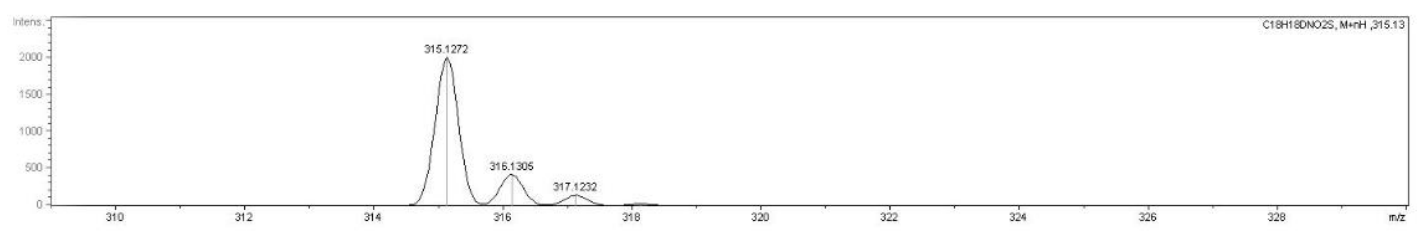

\section{Simulated HRMS of 8a-d}

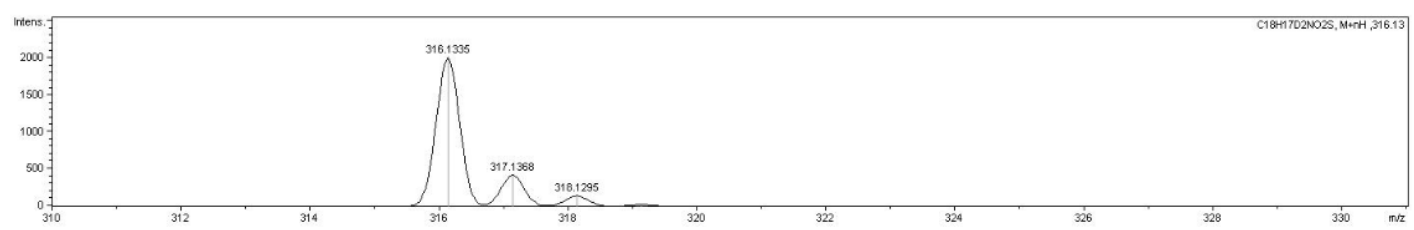

The other reaction was conducted under the standard conditions for $2 \mathrm{~h}$ (eq. 6) and product 6a was isolated as white solid (20.4 mg, $42 \%$ yield). ${ }^{1} \mathbf{H}$ NMR $(500 \mathrm{MHz}$, $\left.\mathrm{CDCl}_{3}\right) \delta 7.72(\mathrm{~d}, J=8.2 \mathrm{~Hz}, 2 \mathrm{H}), 7.62(\mathrm{~d}, J=8.2 \mathrm{~Hz}, 2 \mathrm{H}), 7.37-7.28(\mathrm{~m}, 7 \mathrm{H}), 7.21$ $(\mathrm{d}, J=8.1 \mathrm{~Hz}, 1 \mathrm{H}), 5.96\left(\mathrm{~A}_{1} \mathrm{~B}_{1} \mathrm{X}_{1}, J_{\mathrm{XA}}=4.3, J_{\mathrm{XB}}=1.2 \mathrm{~Hz}, 0.47 \mathrm{H}\right), 5.20(\mathrm{t}, J=6.3$ $\mathrm{Hz}, 1 \mathrm{H}), 4.14\left(\mathrm{~A}_{1} \mathrm{~B}_{1} \mathrm{X}_{1}, J_{\mathrm{AB}}=17.2, J_{\mathrm{AX}}=4.3 \mathrm{~Hz}, 1 \mathrm{H}\right), 4.07\left(\mathrm{~A}_{2} \mathrm{~B}_{2} \mathrm{X}_{2}, J_{\mathrm{AB}}=12.2, J\right.$ $\mathrm{AX}=0 \mathrm{~Hz}, 1 \mathrm{H}), 3.34\left(\mathrm{~A}_{1} \mathrm{~B}_{1} \mathrm{X}_{1}, J_{\mathrm{BA}}=17.2, J_{\mathrm{BX}}=1.2 \mathrm{~Hz}, 1 \mathrm{H}\right), 3.21(\mathrm{~m}, 0.47 \mathrm{H})$, 3.01-2.91 (m, 2H), $2.62\left(\mathrm{~A}_{2} \mathrm{~B}_{2} \mathrm{X}_{2}, J_{\mathrm{BA}}=12.2, J_{\mathrm{BX}}=5.4 \mathrm{~Hz}, 1 \mathrm{H}\right), 2.44(\mathrm{~s}, 3 \mathrm{H}), 2.37$ (s, 3H). HRMS experiment of this sample identified one species: HRMS (ESI) calcd. for $\mathrm{C}_{26} \mathrm{H}_{27} \mathrm{DKN}_{2} \mathrm{O}_{4} \mathrm{~S}_{2}$ [M+K]: 536.1185, found: 536.1186. This species matches the proposed 6a-d and 6a'-d. 


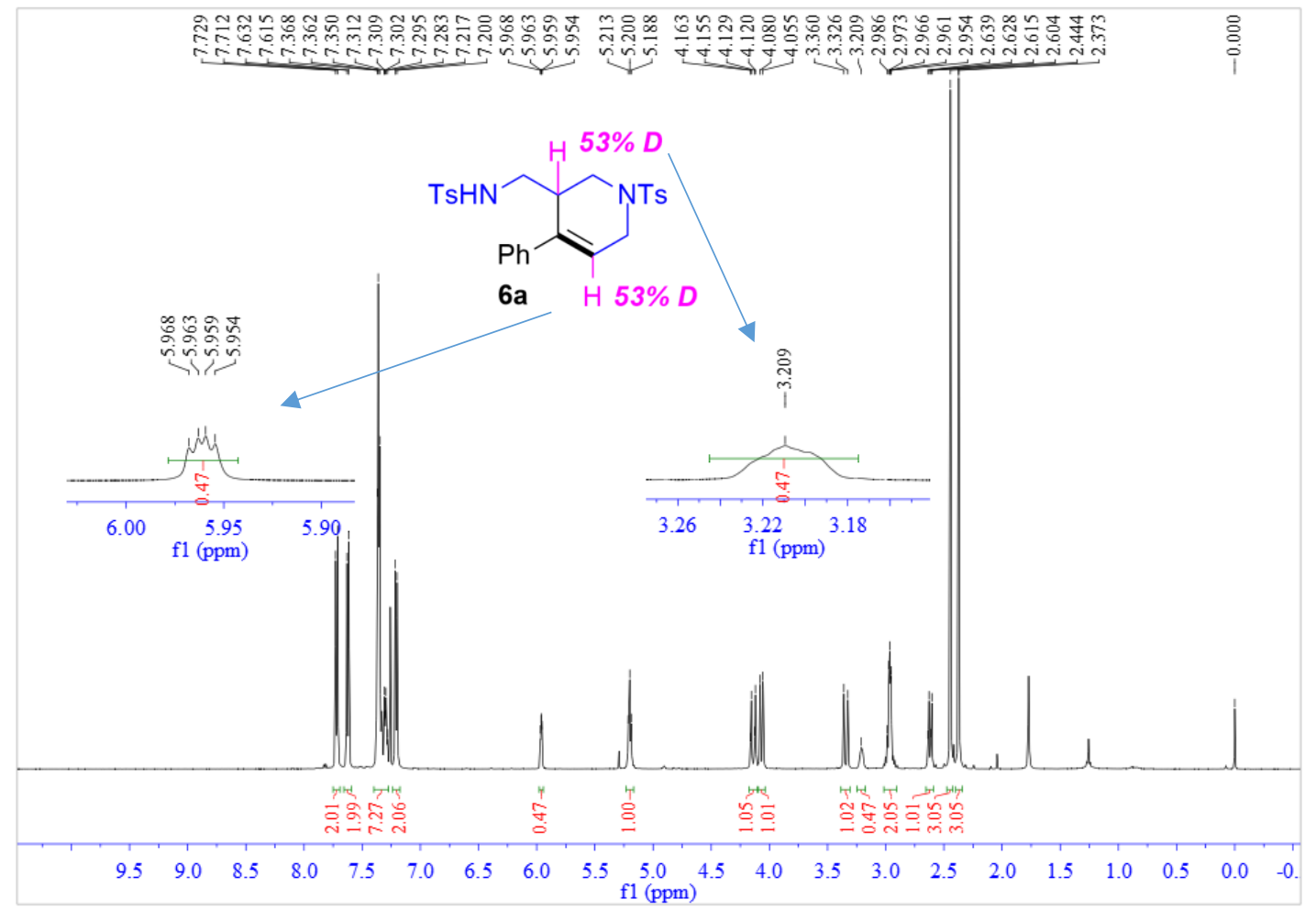

HRMS of $6 \mathbf{a}$ from equation 6 :

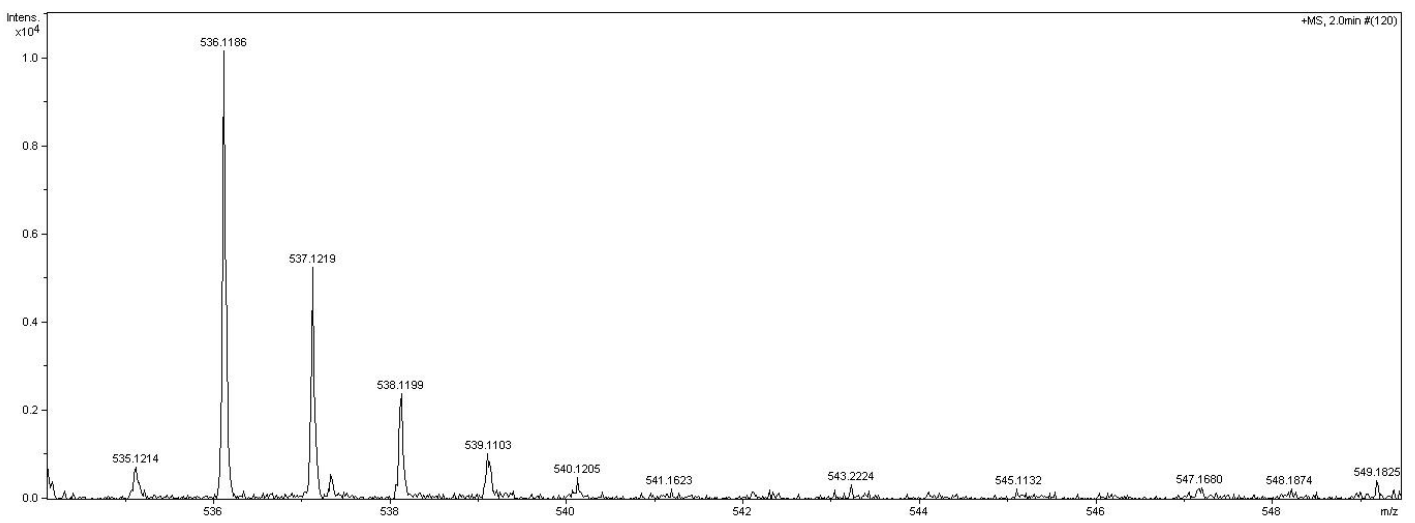

Simulated HRMS of $\mathbf{6 a}-\mathrm{d}$ or $\mathbf{6} \mathbf{a}^{\prime}-\mathrm{d}$

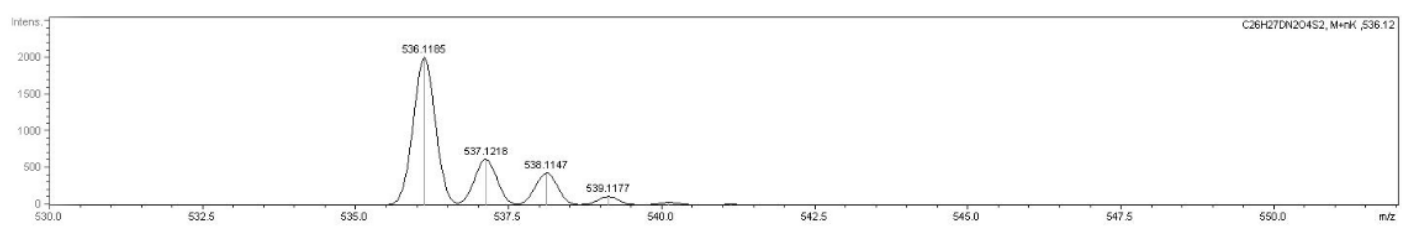




\section{Synthesis of Compounds $8 \mathrm{n}$ and 11}

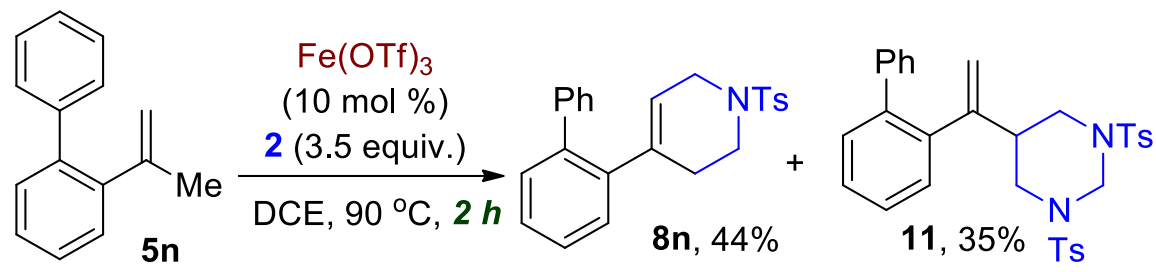

$\alpha$-Methylstyrene derivative 5n (0.1 mmol), bis(tosylamido)methane $(0.1240 \mathrm{~g}, 0.35$ mmol), catalyst $\mathrm{Fe}(\mathrm{OTf})_{3}(10 \mathrm{~mol} \%)$ and $2 \mathrm{~mL} \mathrm{DCE}$ were added to an oven-dried Schlenk tube which was charged with and a magnetic stir bar. The resultant solution was then purged with nitrogen for $1 \mathrm{~min}$ and stirred at $90{ }^{\circ} \mathrm{C}$ for $2 \mathrm{~h}$. After the reaction is done, the reaction mixture was purified by flash chromatography (hexane/ethyl acetate $=10: 1-8: 1)$ and (hexane/ethyl acetate $=6: 1-3: 1)$ to afford the pure product 8n in 44\% yield and $\mathbf{1 1}$ with a bit impurities. The pure compound $\mathbf{1 1}$ was obtained in $35 \%$ yield by washing with small amount of ethanal and hexane.

\section{Spectra of Compounds 8n and 11}

\section{4-([1,1'-biphenyl]-2-yl)-1-tosyl-1,2,3,6-tetrahydropyridine}<smiles>[NH3+]C1CC=C(c2ccccc2-c2ccccc2)CC1</smiles>

The title compound $\mathbf{8 n}$ was prepared according to the general procedure as white solid (16.6 mg, 43\% yield, mp: $139.8-141.7^{\circ} \mathrm{C}$ ). $\mathrm{R}_{f}=0.60$ (hexane/ethyl acetate $2: 1$ ). ${ }^{1} \mathbf{H}$ NMR $\left(500 \mathrm{MHz}, \mathrm{CDCl}_{3}\right) \delta 7.65(\mathrm{~d}, J=8.2 \mathrm{~Hz}, 2 \mathrm{H}), 7.36-7.26(\mathrm{~m}, 10 \mathrm{H}), 7.17-7.10$ (m, 1H), 5.63 (br, 1H), 3.69-3.67 (m, 2H), 2.98 (t, J=5.6 Hz, 2H), 2.44 (s, 3H), 1.991.95 (m, 2H); ${ }^{13}$ C NMR (125 MHz, $\left.\mathrm{CDCl}_{3}\right) \delta 143.5$ (C), 141.5 (C), 140.69 (C), 139.8 (C), $138.4(\mathrm{C}), 133.5(\mathrm{C}), 130.2(\mathrm{CH}), 129.7(2 \mathrm{CH}), 129.3(\mathrm{CH}), 128.8(2 \mathrm{CH}), 128.2$ (2 $\mathrm{CH}), 127.7$ (overlapping $2 \mathrm{CH}$ and $\mathrm{CH}), 127.4(\mathrm{CH}), 127.1(\mathrm{CH}), 122.1(\mathrm{CH})$, $45.1\left(\mathrm{CH}_{2}\right), 43.0\left(\mathrm{CH}_{2}\right), 29.3\left(\mathrm{CH}_{2}\right), 21.6\left(\mathrm{CH}_{3}\right) ;$ HRMS (ESI) calcd. for $\mathrm{C}_{24} \mathrm{H}_{23} \mathrm{KNO}_{2} \mathrm{~S}[\mathrm{M}+\mathrm{K}]: 428.1081$, found: 428.1080 . 


\section{5-(1-([1,1'-biphenyl]-2-yl)vinyl)-1,3-ditosylhexahydropyrimidine}<smiles>C=C(c1ccccc1-c1ccccc1)C1CN(S)C[NH+](S)C1</smiles>

The title compound $\mathbf{1 1}$ was prepared according to the general procedure as white solid (17.2 mg, 30\% yield, mp: $69.8-71.4{ }^{\circ} \mathrm{C}$ ). $\mathrm{R}_{f}=0.50$ (hexane/ethyl acetate $2: 1$ ). ${ }^{1} \mathbf{H}$ NMR $\left(500 \mathrm{MHz}, \mathrm{CDCl}_{3}\right) \delta 7.51(\mathrm{~d}, J=8.2 \mathrm{~Hz}, 4 \mathrm{H}), 7.40-7.29(\mathrm{~m}, 6 \mathrm{H}), 7.26(\mathrm{~d}, J=$ $8.2 \mathrm{~Hz}, 4 \mathrm{H}), 7.18-7.16(\mathrm{~m}, 2 \mathrm{H}), 6.99$ (d, $J=7.5 \mathrm{~Hz}, 1 \mathrm{H}), 5.51(\mathrm{~d}, J=12.4 \mathrm{~Hz}, 1 \mathrm{H})$, $5.15(\mathrm{~s}, 1 \mathrm{H}), 4.96(\mathrm{~s}, 1 \mathrm{H}), 3.47$ (d, $J=12.3 \mathrm{~Hz}, 1 \mathrm{H}), 3.40(\mathrm{dd}, J=12.8,3.4 \mathrm{~Hz}, 2 \mathrm{H})$, $2.45(\mathrm{~s}, 6 \mathrm{H}), 2.31(\mathrm{t}, J=12.3 \mathrm{~Hz}, 2 \mathrm{H}), 1.75(\mathrm{~m}, 1 \mathrm{H}) ;{ }^{13} \mathbf{C ~ N M R}\left(125 \mathrm{MHz}, \mathrm{CDCl}_{3}\right) \delta$ 147.3 (C), 143.1 (2 C), 140.1 (C), 139.5 (C), 138.9 (C), 134.4 (2 C), 129.6 (CH), $129.1(4 \mathrm{CH}), 128.8(\mathrm{CH}), 128.5(2 \mathrm{CH}), 127.4(2 \mathrm{CH}), 127.3(\mathrm{CH}), 126.8(4 \mathrm{CH})$, $126.7(\mathrm{CH}), 126.6(\mathrm{CH}), 116.5\left(\mathrm{CH}_{2}\right), 60.0\left(\mathrm{CH}_{2}\right), 48.7\left(2 \mathrm{CH}_{2}\right), 37.7(\mathrm{CH}), 20.9(2$ $\mathrm{CH}_{3}$ ); HRMS (ESI) calcd. for $\mathrm{C}_{32} \mathrm{H}_{33} \mathrm{~N}_{2} \mathrm{O}_{4} \mathrm{~S}_{2}[\mathrm{M}+\mathrm{H}]: 573.1876$, found: 573.1873.

\section{Synthesis of Compounds 12a-c}

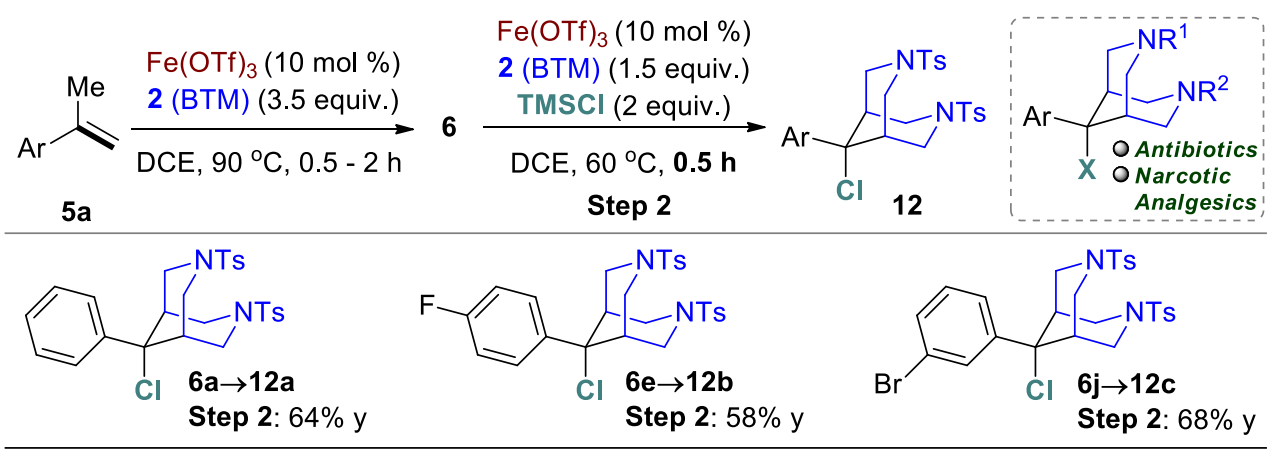

An oven-dried Schlenk tube was charged with bis(tosylamido)methane $(0.1240 \mathrm{~g}$, $0.35 \mathrm{mmol})$, catalyst $\mathrm{Fe}(\mathrm{OTf})_{3}(10 \mathrm{~mol} \%)$ and a magnetic stir bar. The Schlenk tube was then evacuated and back filled with nitrogen. The Teflon screw cap was replaced with a rubber septum. $\alpha$-Methylstyrene $5 \mathbf{a}(12.1 \mathrm{mg}, 0.1 \mathrm{mmol})$ was added and followed by $2 \mathrm{~mL}$ DCE. The Schlenk tube was then purged with nitrogen for $1 \mathrm{~min}$ and the rubber septum was replaced with a Teflon screw cap. The reaction mixture was then stirred at $90{ }^{\circ} \mathrm{C}$ for $0.5 \mathrm{~h}$. After the reaction is done, the reaction mixture 
was purified by flash chromatography (hexane/ethyl acetate $=6: 1-3: 1$ ). The fractions containing the product were collected and concentrated by rotary evaporation to afford the compound $\mathbf{6 a}$ in $65 \%$ yield.

The compound 6a (49.7 mg, $0.1 \mathrm{mmol})$ and $\mathrm{TMSCl}(21.7 \mathrm{mg}, 0.2 \mathrm{mmol})$ were subjected to the reaction system and reacted at $60{ }^{\circ} \mathrm{C}$ for $0.5 \mathrm{~h}$. After the reaction is done monitored by TLC, the reaction mixture was purified by flash chromatography (hexane/ethyl acetate $=6: 1-3: 1$ ). The fractions containing the product were collected and concentrated by rotary evaporation, furnishing the desired product 12a in $64 \%$ yield.

Compound 12b and compound 12c were synthesized from $\mathbf{6 e}$ and $\mathbf{6 j}$ by using the same method.

\section{Characterization of Products 12a-c}

\section{9-chloro-9-phenyl-3,7-ditosyl-3,7-diazabicyclo[3.3.1]nonane}

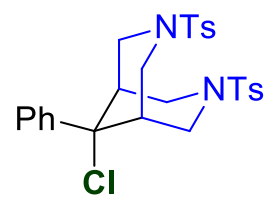

The title compound 12a was prepared as white solid (36 mg, 64\% yield, mp: $224.2-$ $\left.225.5{ }^{\circ} \mathrm{C}\right) . \mathrm{R}_{f}=0.38$ (hexane/ethyl acetate 2:1). ${ }^{1} \mathbf{H} \mathbf{N M R}\left(500 \mathrm{MHz}, \mathrm{CDCl}_{3}\right) \delta 7.73$ $(\mathrm{d}, J=8.2 \mathrm{~Hz}, 2 \mathrm{H}), 7.64(\mathrm{~d}, J=8.2 \mathrm{~Hz}, 2 \mathrm{H}), 7.37-7.32(\mathrm{~m}, 4 \mathrm{H}), 7.31-7.27(\mathrm{~m}, 3 \mathrm{H})$, $7.25(\mathrm{~d}, J=8.1 \mathrm{~Hz}, 2 \mathrm{H}), 3.83(\mathrm{~d}, J=11.4 \mathrm{~Hz}, 2 \mathrm{H}), 3.64$ (d, $J=11.4 \mathrm{~Hz}, 2 \mathrm{H}), 3.55$ (d, $J=11.8 \mathrm{~Hz}, 2 \mathrm{H}), 3.24(\mathrm{~d}, J=11.8 \mathrm{~Hz}, 2 \mathrm{H}), 2.94$ (s, 2H), 2.43 (s, 3H), 2.38 (s, 3H); ${ }^{13} \mathrm{C}$ NMR (125 MHz, $\mathrm{CDCl}_{3}$ ) $\delta 143.8$ (overlapping C and C), 139.2 (C), 133.1 (C), $132.6(\mathrm{C}), 129.8(2 \mathrm{CH}), 129.7(2 \mathrm{CH}), 129.5(2 \mathrm{CH}), 128.8(\mathrm{CH}), 128.0(2 \mathrm{CH})$, $127.9(2 \mathrm{CH}), 124.8(2 \mathrm{CH}), 70.8(\mathrm{C}), 47.2\left(2 \mathrm{CH}_{2}\right), 47.1\left(2 \mathrm{CH}_{2}\right), 37.0(2 \mathrm{CH}), 21.7$ $\left(\mathrm{CH}_{3}\right), 21.6\left(\mathrm{CH}_{3}\right)$; HRMS (ESI) calcd. for $\mathrm{C}_{27} \mathrm{H}_{30} \mathrm{ClN}_{2} \mathrm{O}_{4} \mathrm{~S}_{2}[\mathrm{M}+\mathrm{H}]$ : 545.1330, found: 545.1328. 


\section{9-chloro-9-(4-fluorophenyl)-3,7-ditosyl-3,7-diazabicyclo[3.3.1]nonane}

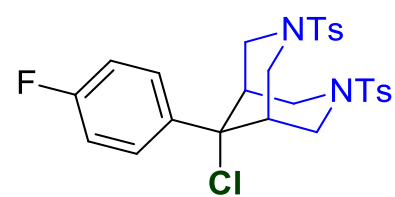

The title compound 12b was prepared as white solid (32.6 mg, 58\% yield, mp: $207.8-$ $\left.209.6{ }^{\circ} \mathrm{C}\right) . \mathrm{R}_{f}=0.36$ (hexane/ethyl acetate 2:1). ${ }^{\mathbf{1}} \mathbf{H} \mathbf{N M R}\left(500 \mathrm{MHz}, \mathrm{CDCl}_{3}\right) \delta 7.73$ $(\mathrm{d}, J=7.6 \mathrm{~Hz}, 2 \mathrm{H}), 7.60(\mathrm{~d}, J=7.6 \mathrm{~Hz}, 2 \mathrm{H}), 7.35(\mathrm{~d}, J=7.6 \mathrm{~Hz}, 2 \mathrm{H}), 7.29-7.24(\mathrm{~m}$, 4H), $7.01(\mathrm{t}, J=8.0 \mathrm{~Hz}, 2 \mathrm{H}), 3.82(\mathrm{~d}, J=11.8 \mathrm{~Hz}, 2 \mathrm{H}), 3.63(\mathrm{~d}, J=11.8 \mathrm{~Hz}, 2 \mathrm{H})$, $3.54(\mathrm{~d}, J=11.6 \mathrm{~Hz}, 2 \mathrm{H}), 3.20(\mathrm{~d}, J=11.6 \mathrm{~Hz}, 2 \mathrm{H}), 2.90(\mathrm{~s}, 2 \mathrm{H}), 2.44(\mathrm{~s}, 3 \mathrm{H}), 2.40$ $(\mathrm{s}, 3 \mathrm{H}) ;{ }^{13} \mathrm{C}$ NMR $\left(125 \mathrm{MHz}, \mathrm{CDCl}_{3}\right) \delta 162.5$ (d, $\left.J=248.8 \mathrm{~Hz}\right)(\mathrm{C}-\mathrm{F}), 144.1$ (C), 144.0 (C), 135.4 (d, $J=3.6$ Hz) (C), 133.0 (C), 132.9 (C), 130.0 (2 CH), 129.9 (2 $\mathrm{CH}), 128.1(4 \mathrm{CH}) 127.0(\mathrm{CH}), 126.9(\mathrm{CH}), 116.5(\mathrm{~d}, J=21.6 \mathrm{~Hz})(2 \mathrm{CH}), 70.4(\mathrm{C})$, $47.4\left(2 \mathrm{CH}_{2}\right), 47.2\left(2 \mathrm{CH}_{2}\right), 37.3(2 \mathrm{CH}), 21.8\left(\mathrm{CH}_{3}\right), 21.7\left(\mathrm{CH}_{3}\right),{ }^{19} \mathbf{F}$ NMR $(470$ $\left.\mathrm{MHz}, \mathrm{CDCl}_{3}\right) \delta-111.95$; HRMS (ESI) calcd. for $\mathrm{C}_{27} \mathrm{H}_{29} \mathrm{ClFN}_{2} \mathrm{O}_{4} \mathrm{~S}_{2} \quad[\mathrm{M}+\mathrm{H}]$ : 563.1236, found: 563.1236 .

\section{9-(3-bromophenyl)-9-chloro-3,7-ditosyl-3,7-diazabicyclo[3.3.1]nonane}

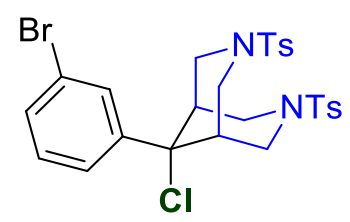

The title compound 12c was prepared as white solid (42.4 mg, 68\% yield, mp: $236.2-$ $\left.237.6{ }^{\circ} \mathrm{C}\right) . \mathrm{R}_{f}=0.35$ (hexane/ethyl acetate $\left.2: 1\right) .{ }^{1} \mathbf{H} \mathbf{~ N M R}\left(500 \mathrm{MHz}, \mathrm{CDCl}_{3}\right) \delta 7.73$ $(\mathrm{d}, J=7.5 \mathrm{~Hz}, 2 \mathrm{H}), 7.62(\mathrm{~d}, J=7.5 \mathrm{~Hz}, 2 \mathrm{H}), 7.40(\mathrm{~b}, 2 \mathrm{H}), 7.35(\mathrm{~d}, J=7.5 \mathrm{~Hz}, 2 \mathrm{H})$, $7.30-7.21(\mathrm{~m}, 4 \mathrm{H}), 3.80(\mathrm{~d}, J=11.7 \mathrm{~Hz}, 2 \mathrm{H}), 3.62(\mathrm{~d}, J=11.7 \mathrm{~Hz}, 2 \mathrm{H}), 3.52(\mathrm{~d}, J$ $=11.4 \mathrm{~Hz}, 2 \mathrm{H}), 3.23(\mathrm{~d}, J=11.4 \mathrm{~Hz}, 2 \mathrm{H}), 2.87(\mathrm{~s}, 2 \mathrm{H}), 2.44(\mathrm{~s}, 3 \mathrm{H}), 2.41(\mathrm{~s}, 3 \mathrm{H}) ;{ }^{13} \mathrm{C}$ NMR (125 MHz, $\left.\mathrm{CDCl}_{3}\right) \delta 143.9(\mathrm{C}), 143.8$ (C), 141.5 (C), 132.7 (C), 132.6 (C), $131.8(\mathrm{CH}), 130.9(\mathrm{CH}), 129.8(2 \mathrm{CH}), 129.7(2 \mathrm{CH}), 128.0(\mathrm{CH}), 127.9(2 \mathrm{CH})$, $127.8(2 \mathrm{CH}), 123.5(\mathrm{C}), 123.3(\mathrm{CH}), 69.8(\mathrm{C}), 47.1\left(2 \mathrm{CH}_{2}\right), 46.9\left(2 \mathrm{CH}_{2}\right), 36.9$ (2 
$\mathrm{CH}), 21.6\left(\mathrm{CH}_{3}\right), 21.5\left(\mathrm{CH}_{3}\right)$; HRMS (ESI) calcd. for $\mathrm{C}_{27} \mathrm{H}_{28} \mathrm{BrClN}_{2} \mathrm{NaO}_{4} \mathrm{~S}_{2}[\mathrm{M}+\mathrm{Na}]$ : 645.0255, found: 645.0256 .

\section{References}

1. Li, Z.Y.; Chaminda Lakmal, H. H.; Cui, X. Enabling Catalytic Arene C-H Amidomethylation via Bis(tosylamido)methane as a Sustainable Formaldimine Releaser. Org. Lett. 2019, 21, 3735-3740.

2. Cussó, O.; Ribas, X.; Lloret-Fillol, J.; Costas, M. Synergistic Interplay of a NonHeme Iron Catalyst and Amino Acid Coligands in $\mathrm{H}_{2} \mathrm{O}_{2}$ Activation for Asymmetric Epoxidation of $\alpha$-Alkyl-Substituted Styrenes. Angew. Chem. Int. Ed. 2015, 54, 27292733.

3. Han, P.; Wang, R.; Wang, D. Z. Electronic Polarizability-Based Stereochemical Model for Sharpless AD Reactions. Tetrahedron 2011, 67, 8873-8878.

4. Chatalova-Sazepin, C.; Wang, Q.; Sammis, G. M.; Zhu, J., Copper-Catalyzed Intermolecular Carboetherification of Unactivated Alkenes by Alkyl Nitriles and Alcohols. Angew. Chem. Int. Ed. 2015, 54, 5443-5446.

5. Fulmer, G. R.; Miller, A. J.; Sherden, N. H.; Gottlieb, H. E.; Nudelman, A.; Stoltz, B. M., Bercaw, J. E.; Goldberg, K. I. NMR Chemical Shifts of Trace Impurities: Common Laboratory Solvents, Organics, and Gases in Deuterated Solvents Relevant to the Organometallic Chemist. Organometallics 2010, 29, 2176-2179.

6. Saint Program included in the package software: APEX3 v2019.1.0.

7. Sadabs, Bruker (2001). Bruker AXS Inc., Madison, Wisconsin, USA.

8. Sheldrick, G. M. SHELXT-Integrated space-group and crystal-structure determination. Acta Cryst. 2015, A71, 3-8. 
9. Sheldrick, G. M. Crystal structure refinement with SHELXL. Acta Cryst. 2015, C71, 3-8.

10. APEX3 v2019, 1.0, AXS Bruker program.

\section{X-ray Crystallography for 6 a}

A colorless prism single crystal of $\mathrm{C}_{26} \mathrm{H}_{28} \mathrm{~N}_{2} \mathrm{O}_{4} \mathrm{~S}_{2}$, approximate dimensions $(0.255 \mathrm{x}$ $0.301 \times 0.443) \mathrm{mm}^{3}$, was selected for the $\mathrm{X}$-ray crystallographic analysis and mounted on a cryoloop using an oil cryoprotectant. The X-ray intensity data was measured at low temperature $(\mathrm{T}=100 \mathrm{~K})$, using a three circles goniometer Kappa geometry with a fixed Kappa angle at $=54.74 \mathrm{deg}$ Bruker AXS D8 Venture, equipped with a Photon 100 CMOS active pixel sensor detector. A monochromatized Molybdenum X-ray radiation $(\lambda=0.71073 \AA)$ was selected for the measurement. Frames were integrated with the Bruker SAINT software ${ }^{6}$ using a narrow-frame algorithm. The integration of the data using a triclinic unit cell yielded a total of 39075 reflections to a maximum $\theta$ angle of $29.87^{\circ}(0.71 \AA$ resolution), of which 6128 were independent (average redundancy 6.376 , completeness $\left.=86.9 \%, \mathrm{R}_{\text {int }}=2.17 \%, \mathrm{R}_{\text {sig }}=1.67 \%\right)$ and 5665 (92.44\%) were greater than $2 \sigma\left(F^{2}\right)$. The final cell constants of $\underline{a}=9.950(2) \AA ⿻$ 10.420(2) $\AA, \underline{\mathrm{c}}=13.328(3) \AA, \alpha=73.734(7)^{\circ}, \beta=83.867(6)^{\circ}, \gamma=67.198(6)^{\circ}$, volume $=1222.9(4) \AA^{3}$, are based upon the refinement of the XYZ-centroids of 1591 reflections above $20 \sigma$ (I) with $4.391^{\circ}<2 \theta<51.75^{\circ}$. Data were corrected for absorption effects using the Multi-Scan method implemented in the program (SADABS) $)^{7}$. The ratio of minimum to maximum apparent transmission was 0.934 . The calculated minimum and maximum transmission coefficients (based on crystal size) are 0.8960 and 0.9380 . The structure was solved in a triclinic unit cell using the Bruker SHELXT Software Package ${ }^{8}$, using the centrosymmetric space group P -1, with $\mathrm{Z}=2$ for the formula unit, $\mathrm{C}_{26} \mathrm{H}_{28} \mathrm{~N}_{2} \mathrm{O}_{4} \mathrm{~S}_{2}$. Refinement of the structure was carried out by least squares procedures on weighted $\mathrm{F}^{2}$ values using the SHELXTL2018/3 $3^{9}$ included in the APEX3 v2019, 1.0, AXS Bruker program ${ }^{10}$. Hydrogen atoms were localized on difference Fourier maps but then introduced in the refinement as fixed contributors in idealized geometry with an isotropic thermal parameters fixed at $20 \%$ higher than those carbons atoms they were connected. Excepted for the $\mathrm{H}$ atom labelled: $\mathrm{H} 2 \mathrm{~N}$ connected to the nitrogen atom: $\mathrm{N} 2$, that was isotropically refined using a free variable. The final anisotropic full-matrix least-squares refinement on $\mathrm{F}^{2}$ with 314 variables converged at $\mathrm{R} 1=3.08 \%$, for the observed data and $\mathrm{wR} 2=8.28 \%$ for all data. The goodness-of-fit: GOF was 1.045. The largest peak in the final difference electron density synthesis was $0.407 \mathrm{e}^{-} / \AA^{3}$ and the largest hole was- 0.424 
$\mathrm{e}^{-} / \AA^{3}$ with an RMS deviation of $0.049 \mathrm{e}^{-} / \AA^{3}$. On the basis of the final model, the calculated density was $1.349 \mathrm{~g} / \mathrm{cm}^{3}$ and $\mathrm{F}(000), 524 \mathrm{e}^{-}$.
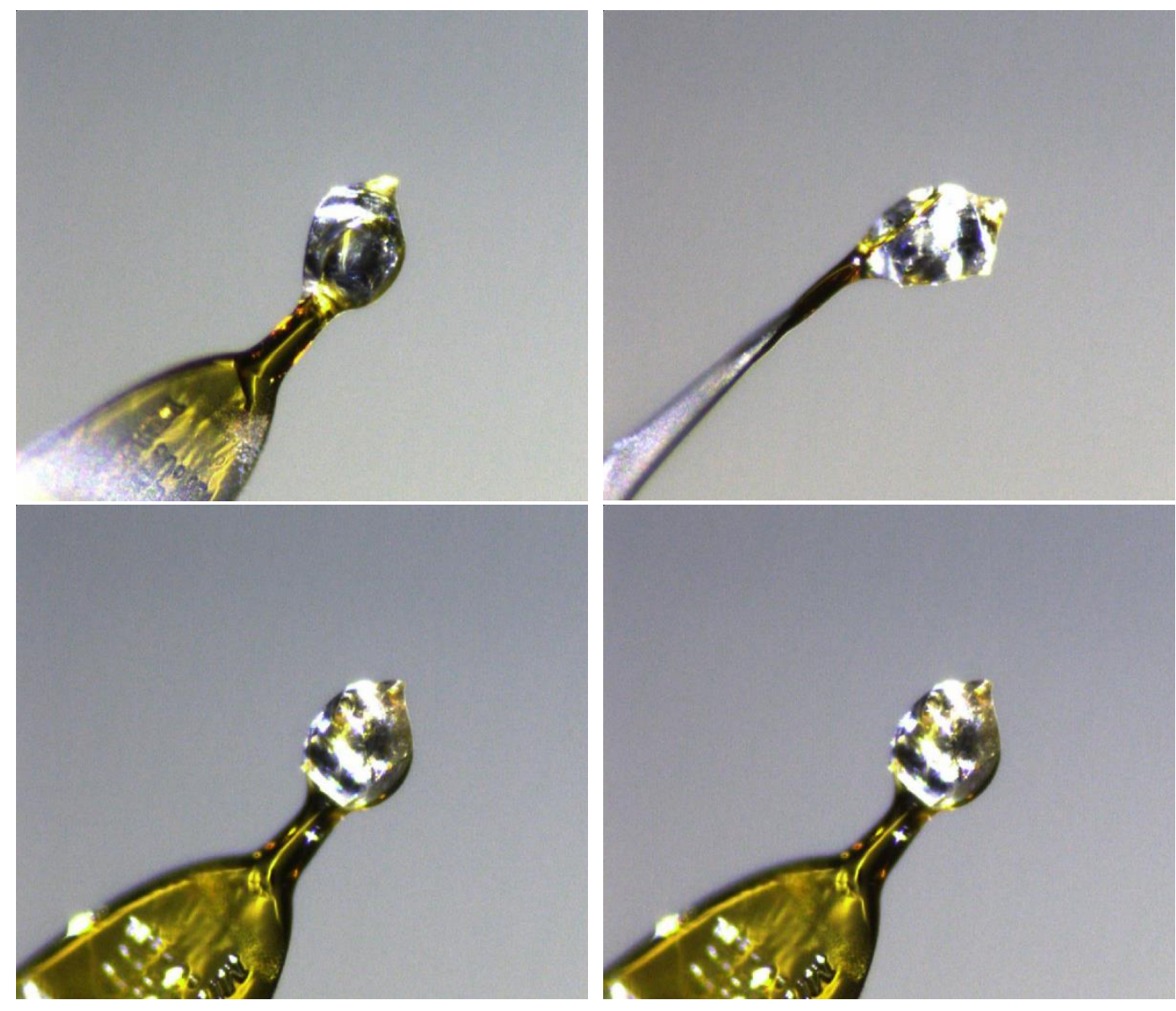

\section{Crystal's views:}



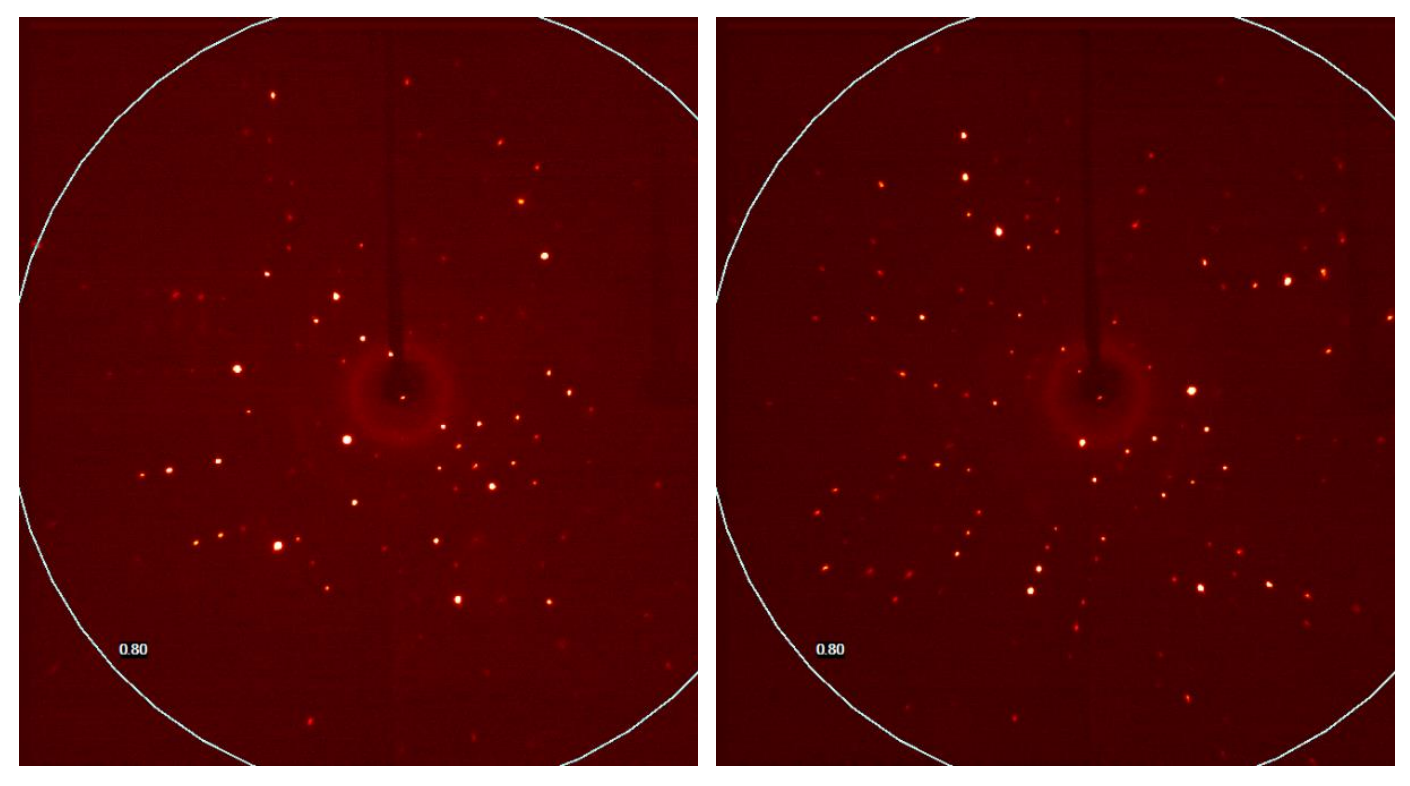

Crystal's resolution:

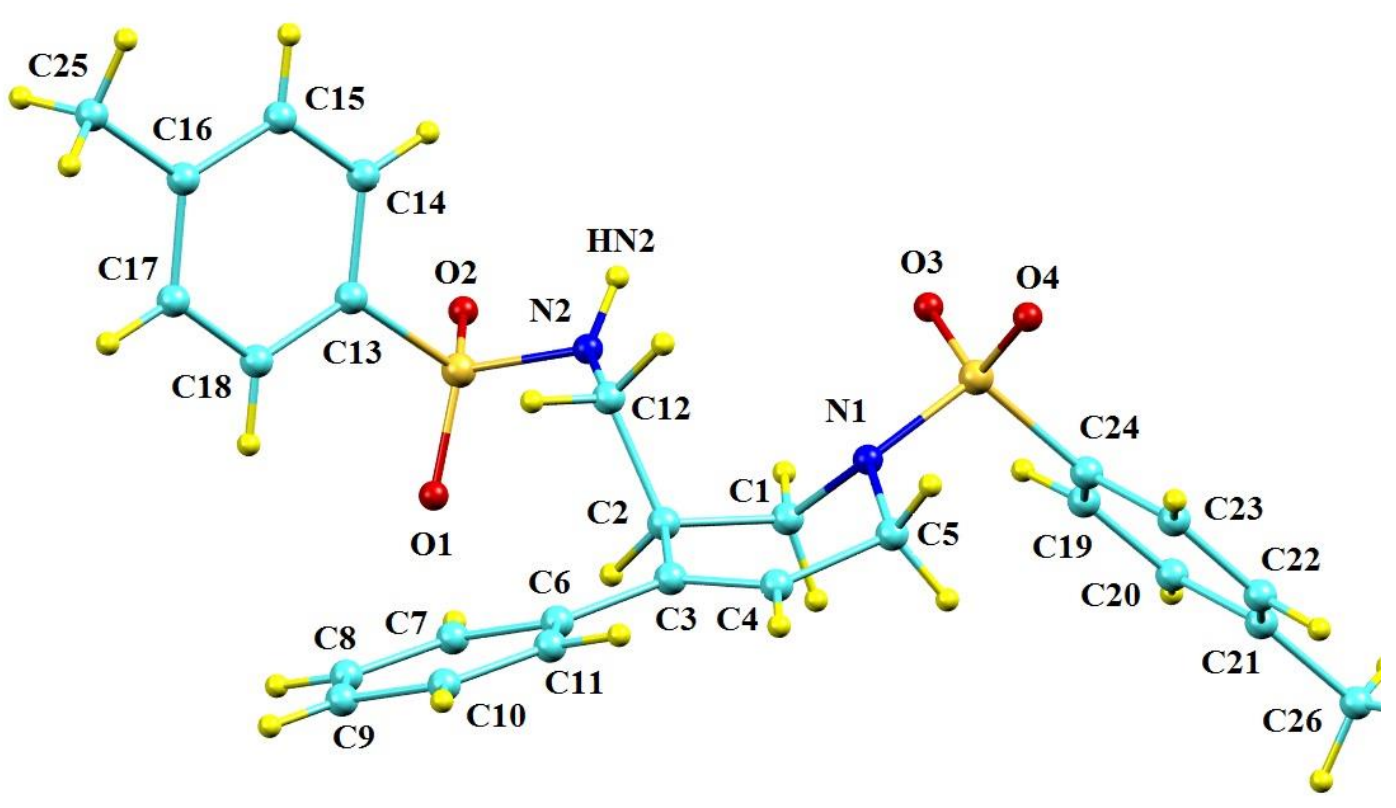

Asymmetric unit's view: 
Table S1. Sample and crystal data for MSU_032.

\begin{tabular}{|c|c|c|}
\hline Identification code & \multicolumn{2}{|l|}{ MSU_032 } \\
\hline Chemical formula & \multicolumn{2}{|l|}{$\mathrm{C}_{26} \mathrm{H}_{28} \mathrm{~N}_{2} \mathrm{O}_{4} \mathrm{~S}_{2}$} \\
\hline Formula weight & \multicolumn{2}{|l|}{$496.62 \mathrm{~g} / \mathrm{mol}$} \\
\hline Temperature & \multicolumn{2}{|l|}{$100(2) \mathrm{K}$} \\
\hline Wavelength & \multicolumn{2}{|l|}{$0.71073 \AA$} \\
\hline Crystal size & \multicolumn{2}{|c|}{$(0.255 \times 0.301 \times 0.443) \mathrm{mm}^{3}$} \\
\hline Crystal system & \multicolumn{2}{|l|}{ triclinic } \\
\hline Space group & \multicolumn{2}{|l|}{$\mathrm{P}-1$} \\
\hline \multirow[t]{3}{*}{ Unit cell dimensions } & $a=9.950(2) \AA$ & $\alpha=73.734(7)^{\circ}$ \\
\hline & $\mathrm{b}=10.420(2) \AA$ & $\beta=83.867(6)^{\circ}$ \\
\hline & $c=13.328(3) \AA$ & $\gamma=67.198(6)^{\circ}$ \\
\hline Volume & \multicolumn{2}{|l|}{$1222.9(4) \AA^{3}$} \\
\hline $\mathbf{Z}$ & \multicolumn{2}{|l|}{2} \\
\hline Density (calculated) & \multicolumn{2}{|l|}{$1.349 \mathrm{~g} / \mathrm{cm}^{3}$} \\
\hline Absorption coefficient & \multicolumn{2}{|l|}{$0.253 \mathrm{~mm}^{-1}$} \\
\hline$F(000)$ & \multicolumn{2}{|l|}{524} \\
\hline
\end{tabular}

Table S2. Data collection and structure refinement for MSU_032.

$\begin{aligned} & \text { Theta range for data } \\ & \text { collection }\end{aligned}$
$\begin{array}{ll}\text { Index ranges } & -13<=\mathrm{h}<=13,-14<=\mathrm{k}<=14,- \\ 18<=\mathrm{l}<=18\end{array}$


Reflections collected 39075

Independent

reflections

$6128[\mathrm{R}(\mathrm{int})=0.0217]$

Coverage of

independent $\quad 86.9 \%$

reflections

Absorption
correction

Max. and min.

transmission

0.9380 and 0.8960

Structure solution

technique

direct methods

Structure solution

program

SHELXT 2014/5 (Sheldrick, 2014)

Refinement method Full-matrix least-squares on $\mathrm{F}^{2}$

Refinement

program

SHELXL-2018/3 (Sheldrick, 2018)

Function minimized $\Sigma \mathrm{w}\left(\mathrm{F}_{\mathrm{o}}{ }^{2}-\mathrm{F}_{\mathrm{c}}{ }^{2}\right)^{2}$

Data / restraints /

parameters

$6128 / 0 / 314$

Goodness-of-fit on

$\mathbf{F}^{2}$

1.045

$\Delta / \sigma_{\max }$

0.001

Final $\mathbf{R}$ indices

5665 data;

$\mathrm{R} 1=0.0308, \mathrm{wR} 2=$

$\mathrm{I}>2 \sigma(\mathrm{I})$

0.0808

all data

$\mathrm{R} 1=0.0336, \mathrm{wR} 2=$ 0.0828

Weighting scheme

$\mathrm{w}=1 /\left[\sigma^{2}\left(\mathrm{~F}_{\mathrm{o}}{ }^{2}\right)+(0.0364 \mathrm{P})^{2}+0.6937 \mathrm{P}\right]$

where $\mathrm{P}=\left(\mathrm{F}_{\mathrm{o}}{ }^{2}+2 \mathrm{~F}_{\mathrm{c}}{ }^{2}\right) / 3$ 
Extinction

coefficient

$0.0055(9)$

Largest diff. peak and hole

0.407 and $-0.424 \mathrm{e}^{-3}$

$\begin{array}{ll}\text { R.M.S. deviation } & 0.049 \mathrm{e}^{-3}\end{array}$

Table S3. Atomic coordinates and equivalent isotropic atomic displacement parameters $\left(\AA^{2}\right)$ for $M S U \_032$.

$U(e q)$ is defined as one third of the trace of the orthogonalized $U_{i j}$ tensor.
$\mathbf{x} / \mathbf{a}$
$\mathbf{y} / \mathbf{b}$
z/c
U(eq)

S1 $\quad 0.51612(3)$

$0.10153(3)$

$0.28965(2)$

$0.01385(7)$

O1 $\quad 0.55009(9)$

0.09106(9)

$0.39421(6)$

0.01929(17)

O2 0.61261(9)

0.99955(9)

$0.23562(7)$

$0.02115(18)$

S2 $\quad 0.60530(3)$

$0.65318(3)$

$0.06484(2)$

$0.01760(8)$

O3 $0.65446(10)$

$0.52521(11)$

$0.02820(7)$

$0.0263(2)$

O4 0.49929(10)

$0.78379(11)$

$0.00373(7)$

$0.02424(19)$

$\mathrm{N} 1 \quad 0.53363(10)$

$0.61693(10)$

$0.17971(7)$

$0.01525(18)$

$\mathrm{N} 2 \quad 0.50403(10)$

$0.25976(10)$

$0.21827(7)$

$0.01459(18)$

C1 0.60889(12)

$0.47735(12)$

$0.25445(9)$

$0.0160(2)$

C2 $0.49155(12)$

$0.43150(11)$

$0.31987(8)$

$0.0138(2)$

C3 0.38764(12)

$0.55165(12)$

$0.36636(8)$

$0.0144(2)$

C4 0.38311(12)

$0.68680(12)$

$0.32605(9)$

$0.0165(2)$

C5 $0.46539(12)$

$0.73478(12)$

$0.23118(9)$

$0.0165(2)$ 


\section{$\mathbf{x} / \mathbf{a}$}

C6 $0.29292(12)$

C7 0.32021(13)

C8 0.22972(14)

C9 $0.11046(14)$

C10 0.08124(14)

C11 0.17080(13)

C12 0.40806(12)

C13 0.34018(12)

C14 0.27417(13)

C15 0.13581(14)

C16 0.06285(13)

C17 0.13258(14)

C18 0.27028(13)

C19 0.75972(12)

C20 0.74220(13)

C21 0.86503(13)

C22 0.00378(13)

C23 0.01818(13)

C24 0.89679(13)

C25 0.91326(15)

C26 0.13662(15)

\begin{abstract}
$\mathbf{y} / \mathbf{b}$
\end{abstract}
$\mathbf{z} / \mathbf{c}$

$$
\text { 0.51401(12) }
$$

$0.36995(13)$

$0.33484(14)$

$0.44237(15)$

$0.58569(15)$

$0.62088(13)$

$0.38754(11)$

0.09507(11)

0.11883(13)

$0.11520(14)$

$0.08562(13)$

$0.06112(14)$

$0.06634(13)$

0.69074(13)

0.83027(13)

0.85794(13)

0.74976(13)

$0.61030(14)$

$0.57980(13)$

$0.07925(18)$

0.78109(16)
$0.45545(8)$

0.51085(9)

0.59256(9)

0.62093(9)

$0.56715(10)$

0.48523(9)

0.25344(8)

0.29297(8)

0.19897(9)

$0.20015(10)$

$0.29410(10)$

$0.38675(10)$

$0.38745(9)$

0.07928(8)

$0.07872(9)$

0.09076(9)

$0.10126(9)$

$0.10242(9)$

0.09251(9)

$0.29513(12)$

$0.11248(11)$
$\mathbf{U}(\mathbf{e q})$

$0.0158(2)$

0.0197(2)

0.0229(2)

0.0241(3)

0.0244(3)

0.0204(2)

0.0142(2)

0.0143(2)

$0.0198(2)$

0.0224(2)

0.0216(2)

0.0231(2)

0.0191(2)

$0.0160(2)$

0.0183(2)

0.0197(2)

0.0190(2)

$0.0199(2)$

0.0189(2)

0.0340(3)

0.0290(3) 
Table S4. Bond lengths (Å) for MSU_032.

\begin{tabular}{|c|c|c|c|}
\hline S1-O1 & $1.4334(9)$ & $\mathrm{S} 1-\mathrm{O} 2$ & $1.4352(9)$ \\
\hline S1-N2 & $1.6218(10)$ & S1-C13 & $1.7733(12)$ \\
\hline $\mathrm{S} 2-\mathrm{O} 3$ & $1.4366(10)$ & $\mathrm{S} 2-\mathrm{O} 4$ & $1.4416(10)$ \\
\hline S2-N1 & $1.6269(10)$ & S2-C19 & $1.7671(12)$ \\
\hline N1-C5 & $1.4715(14)$ & $\mathrm{N} 1-\mathrm{C} 1$ & $1.4738(14)$ \\
\hline $\mathrm{N} 2-\mathrm{C} 12$ & $1.4740(14)$ & N2-HN2 & $0.887(18)$ \\
\hline $\mathrm{C} 1-\mathrm{C} 2$ & $1.5274(15)$ & C1-H1A & 0.99 \\
\hline C1-H1B & 0.99 & $\mathrm{C} 2-\mathrm{C} 3$ & $1.5214(15)$ \\
\hline $\mathrm{C} 2-\mathrm{C} 12$ & $1.5372(14)$ & $\mathrm{C} 2-\mathrm{H} 2$ & 1.0 \\
\hline $\mathrm{C} 3-\mathrm{C} 4$ & $1.3438(16)$ & C3-C6 & $1.4961(15)$ \\
\hline $\mathrm{C} 4-\mathrm{C} 5$ & $1.5036(15)$ & $\mathrm{C} 4-\mathrm{H} 4$ & 0.95 \\
\hline C5-H5A & 0.99 & C5-H5B & 0.99 \\
\hline C6-C11 & $1.4036(16)$ & C6-C7 & $1.4066(16)$ \\
\hline C7-C8 & $1.3975(17)$ & $\mathrm{C} 7-\mathrm{H} 7$ & 0.95 \\
\hline C8-C9 & $1.3847(19)$ & $\mathrm{C} 8-\mathrm{H} 8$ & 0.95 \\
\hline C9-C10 & $1.3917(19)$ & C9-H9 & 0.95 \\
\hline C10-C11 & $1.3935(17)$ & C10-H10 & 0.95 \\
\hline C11-H11 & 0.95 & $\mathrm{C} 12-\mathrm{H} 12 \mathrm{~A}$ & 0.99 \\
\hline C12-H12B & 0.99 & C13-C18 & $1.3863(15)$ \\
\hline C13-C14 & $1.3922(15)$ & C14-C15 & $1.3902(17)$ \\
\hline C14-H14 & 0.95 & $\mathrm{C} 15-\mathrm{C} 16$ & $1.3974(18)$ \\
\hline C15-H15 & 0.95 & C16-C17 & $1.3931(18)$ \\
\hline
\end{tabular}




$\begin{array}{llll}\mathrm{C} 16-\mathrm{C} 25 & 1.5138(17) & \mathrm{C} 17-\mathrm{C} 18 & 1.3933(17) \\ \mathrm{C} 17-\mathrm{H} 17 & 0.95 & \mathrm{C} 18-\mathrm{H} 18 & 0.95 \\ \mathrm{C} 19-\mathrm{C} 20 & 1.3936(17) & \mathrm{C} 19-\mathrm{C} 24 & 1.3938(17) \\ \mathrm{C} 20-\mathrm{C} 21 & 1.3942(16) & \mathrm{C} 20-\mathrm{H} 20 & 0.95 \\ \mathrm{C} 21-\mathrm{C} 22 & 1.3939(18) & \mathrm{C} 21-\mathrm{H} 21 & 0.95 \\ \mathrm{C} 22-\mathrm{C} 23 & 1.3997(17) & \mathrm{C} 22-\mathrm{C} 26 & 1.5100(16) \\ \mathrm{C} 23-\mathrm{C} 24 & 1.3902(16) & \mathrm{C} 23-\mathrm{H} 23 & 0.95 \\ \mathrm{C} 24-\mathrm{H} 24 & 0.95 & \mathrm{C} 25-\mathrm{H} 25 \mathrm{~A} & 0.98 \\ \mathrm{C} 25-\mathrm{H} 25 \mathrm{~B} & 0.98 & \mathrm{C} 25-\mathrm{H} 25 \mathrm{C} & 0.98 \\ \mathrm{C} 26-\mathrm{H} 26 \mathrm{~A} & 0.98 & \mathrm{C} 26-\mathrm{H} 26 \mathrm{~B} & 0.98 \\ \mathrm{C} 26-\mathrm{H} 26 \mathrm{C} & 0.98 & & \end{array}$

Table S5. Bond angles $\left(^{\circ}\right)$ for MSU_032.

\begin{tabular}{llll}
\hline & & & \\
\hline O1-S1-O2 & $119.68(5)$ & O1-S1-N2 & $107.65(5)$ \\
O2-S1-N2 & $106.40(5)$ & O1-S1-C13 & $107.03(5)$ \\
O2-S1-C13 & $108.46(5)$ & N2-S1-C13 & $107.03(5)$ \\
O3-S2-O4 & $118.86(6)$ & O3-S2-N1 & $107.40(5)$ \\
O4-S2-N1 & $106.63(5)$ & O3-S2-C19 & $107.88(6)$ \\
O4-S2-C19 & $107.49(6)$ & N1-S2-C19 & $108.20(5)$ \\
C5-N1-C1 & $112.39(9)$ & C5-N1-S2 & $117.93(8)$ \\
C1-N1-S2 & $118.66(8)$ & C12-N2-S1 & $118.61(7)$ \\
C12-N2-HN2 & $118.0(11)$ & S1-N2-HN2 & $112.0(11)$ \\
N1-C1-C2 & $107.19(9)$ & N1-C1-H1A & 110.3
\end{tabular}




\begin{tabular}{|c|c|c|c|}
\hline C2-C1-H1A & 110.3 & N1-C1-H1B & 110.3 \\
\hline C2-C1-H1B & 110.3 & H1A-C1-H1B & 108.5 \\
\hline $\mathrm{C} 3-\mathrm{C} 2-\mathrm{C} 1$ & $110.75(9)$ & $\mathrm{C} 3-\mathrm{C} 2-\mathrm{C} 12$ & $110.78(9)$ \\
\hline C1-C2-C12 & $110.38(9)$ & $\mathrm{C} 3-\mathrm{C} 2-\mathrm{H} 2$ & 108.3 \\
\hline $\mathrm{C} 1-\mathrm{C} 2-\mathrm{H} 2$ & 108.3 & $\mathrm{C} 12-\mathrm{C} 2-\mathrm{H} 2$ & 108.3 \\
\hline C4-C3-C6 & $122.47(10)$ & $\mathrm{C} 4-\mathrm{C} 3-\mathrm{C} 2$ & $119.38(10)$ \\
\hline $\mathrm{C} 6-\mathrm{C} 3-\mathrm{C} 2$ & $118.15(9)$ & $\mathrm{C} 3-\mathrm{C} 4-\mathrm{C} 5$ & $125.37(10)$ \\
\hline C3-C4-H4 & 117.3 & $\mathrm{C} 5-\mathrm{C} 4-\mathrm{H} 4$ & 117.3 \\
\hline $\mathrm{N} 1-\mathrm{C} 5-\mathrm{C} 4$ & $109.49(9)$ & N1-C5-H5A & 109.8 \\
\hline C4-C5-H5A & 109.8 & N1-C5-H5B & 109.8 \\
\hline C4-C5-H5B & 109.8 & H5A-C5-H5B & 108.2 \\
\hline C11-C6-C7 & $117.29(11)$ & C11-C6-C3 & $121.28(10)$ \\
\hline C7-C6-C3 & $121.40(10)$ & C8-C7-C6 & $121.30(11)$ \\
\hline C8-C7-H7 & 119.4 & C6-C7-H7 & 119.4 \\
\hline C9-C8-C7 & $120.29(12)$ & C9-C8-H8 & 119.9 \\
\hline $\mathrm{C} 7-\mathrm{C} 8-\mathrm{H} 8$ & 119.9 & C8-C9-C10 & $119.42(11)$ \\
\hline C8-C9-H9 & 120.3 & C10-C9-H9 & 120.3 \\
\hline C9-C10-C11 & $120.38(12)$ & C9-C10-H10 & 119.8 \\
\hline C11-C10-H10 & 119.8 & C10-C11-C6 & $121.31(12)$ \\
\hline C10-C11-H11 & 119.3 & C6-C11-H11 & 119.3 \\
\hline N2-C12-C2 & $111.55(9)$ & N2-C12-H12A & 109.3 \\
\hline C2-C12-H12A & 109.3 & N2-C12-H12B & 109.3 \\
\hline C2-C12-H12B & 109.3 & $\mathrm{H} 12 \mathrm{~A}-\mathrm{C} 12-\mathrm{H} 12 \mathrm{~B}$ & 108.0 \\
\hline C18-C13-C14 & $120.80(10)$ & C18-C13-S1 & $120.52(8)$ \\
\hline C14-C13-S1 & $118.68(8)$ & C15-C14-C13 & $119.40(11)$ \\
\hline
\end{tabular}




\begin{tabular}{|c|c|c|c|}
\hline C15-C14-H14 & 120.3 & C13-C14-H14 & 120.3 \\
\hline C14-C15-C16 & 121.19(11) & C14-C15-H15 & 119.4 \\
\hline C16-C15-H15 & 119.4 & C17-C16-C15 & $117.90(11)$ \\
\hline C17-C16-C25 & $121.08(12)$ & C15-C16-C25 & $121.02(12)$ \\
\hline C16-C17-C18 & $121.95(11)$ & C16-C17-H17 & 119.0 \\
\hline C18-C17-H17 & 119.0 & C13-C18-C17 & $118.75(11)$ \\
\hline C13-C18-H18 & 120.6 & C17-C18-H18 & 120.6 \\
\hline C20-C19-C24 & $121.10(10)$ & C20-C19-S2 & 119.48(9) \\
\hline C24-C19-S2 & 119.41(9) & C19-C20-C21 & $118.77(11)$ \\
\hline C19-C20-H20 & 120.6 & C21-C20-H20 & 120.6 \\
\hline C22-C21-C20 & $121.23(11)$ & C22-C21-H21 & 119.4 \\
\hline C20-C21-H21 & 119.4 & $\mathrm{C} 21-\mathrm{C} 22-\mathrm{C} 23$ & $118.82(11)$ \\
\hline C21-C22-C26 & $120.95(11)$ & $\mathrm{C} 23-\mathrm{C} 22-\mathrm{C} 26$ & $120.23(11)$ \\
\hline C24-C23-C22 & $120.85(11)$ & $\mathrm{C} 24-\mathrm{C} 23-\mathrm{H} 23$ & 119.6 \\
\hline C22-C23-H23 & 119.6 & C23-C24-C19 & $119.19(11)$ \\
\hline C23-C24-H24 & 120.4 & C19-C24-H24 & 120.4 \\
\hline $\mathrm{C} 16-\mathrm{C} 25-\mathrm{H} 25 \mathrm{~A}$ & 109.5 & $\mathrm{C} 16-\mathrm{C} 25-\mathrm{H} 25 \mathrm{~B}$ & 109.5 \\
\hline $\begin{array}{l}\mathrm{H} 25 \mathrm{~A}-\mathrm{C} 25- \\
\mathrm{H} 25 \mathrm{~B}\end{array}$ & 109.5 & $\mathrm{C} 16-\mathrm{C} 25-\mathrm{H} 25 \mathrm{C}$ & 109.5 \\
\hline $\begin{array}{l}\mathrm{H} 25 \mathrm{~A}-\mathrm{C} 25- \\
\mathrm{H} 25 \mathrm{C}\end{array}$ & 109.5 & $\mathrm{H} 25 \mathrm{~B}-\mathrm{C} 25-\mathrm{H} 25 \mathrm{C}$ & 109.5 \\
\hline $\mathrm{C} 22-\mathrm{C} 26-\mathrm{H} 26 \mathrm{~A}$ & 109.5 & $\mathrm{C} 22-\mathrm{C} 26-\mathrm{H} 26 \mathrm{~B}$ & 109.5 \\
\hline $\begin{array}{l}\text { H26A-C26- } \\
\text { H26B }\end{array}$ & 109.5 & $\mathrm{C} 22-\mathrm{C} 26-\mathrm{H} 26 \mathrm{C}$ & 109.5 \\
\hline $\begin{array}{l}\mathrm{H} 26 \mathrm{~A}-\mathrm{C} 26- \\
\mathrm{H} 26 \mathrm{C}\end{array}$ & 109.5 & $\mathrm{H} 26 \mathrm{~B}-\mathrm{C} 26-\mathrm{H} 26 \mathrm{C}$ & 109.5 \\
\hline
\end{tabular}


Table S6. Torsion angles $\left(^{\circ}\right)$ for MSU_032.

\begin{tabular}{|c|c|c|c|}
\hline O3-S2-N1-C5 & $-175.80(8)$ & O4-S2-N1-C5 & $-47.38(9)$ \\
\hline C19-S2-N1-C5 & $67.99(9)$ & O3-S2-N1-C1 & $42.83(9)$ \\
\hline O4-S2-N1-C1 & $171.24(8)$ & C19-S2-N1-C1 & $-73.39(9)$ \\
\hline O1-S1-N2-C12 & $-52.06(9)$ & O2-S1-N2-C12 & $178.50(8)$ \\
\hline C13-S1-N2-C12 & $62.69(9)$ & C5-N1-C1-C2 & $69.77(11)$ \\
\hline $\mathrm{S} 2-\mathrm{N} 1-\mathrm{C} 1-\mathrm{C} 2$ & $-146.85(7)$ & N1-C1-C2-C3 & $-52.11(11)$ \\
\hline N1-C1-C2-C12 & $70.95(11)$ & $\mathrm{C} 1-\mathrm{C} 2-\mathrm{C} 3-\mathrm{C} 4$ & $17.46(14)$ \\
\hline C12-C2-C3-C4 & $-105.37(12)$ & $\mathrm{C} 1-\mathrm{C} 2-\mathrm{C} 3-\mathrm{C} 6$ & $-163.05(9)$ \\
\hline C12-C2-C3-C6 & $74.12(12)$ & C6-C3-C4-C5 & $-174.53(10)$ \\
\hline C2-C3-C4-C5 & $4.94(17)$ & C1-N1-C5-C4 & $-46.44(12)$ \\
\hline S2-N1-C5-C4 & $169.89(8)$ & C3-C4-C5-N1 & $8.69(16)$ \\
\hline C4-C3-C6-C11 & $15.60(17)$ & C2-C3-C6-C11 & $-163.87(10)$ \\
\hline C4-C3-C6-C7 & $-166.19(11)$ & C2-C3-C6-C7 & $14.34(15)$ \\
\hline C11-C6-C7-C8 & $-0.30(17)$ & C3-C6-C7-C8 & $-178.58(10)$ \\
\hline C6-C7-C8-C9 & $-0.14(18)$ & C7-C8-C9-C10 & $0.19(18)$ \\
\hline C8-C9-C10-C11 & $0.21(19)$ & C9-C10-C11-C6 & $-0.68(19)$ \\
\hline C7-C6-C11-C10 & $0.71(17)$ & C3-C6-C11-C10 & 178.99(11) \\
\hline S1-N2-C12-C2 & $92.11(10)$ & $\mathrm{C} 3-\mathrm{C} 2-\mathrm{C} 12-\mathrm{N} 2$ & $-173.09(9)$ \\
\hline $\mathrm{C} 1-\mathrm{C} 2-\mathrm{C} 12-\mathrm{N} 2$ & $63.86(11)$ & O1-S1-C13-C18 & $-7.07(11)$ \\
\hline O2-S1-C13-C18 & $123.33(10)$ & N2-S1-C13-C18 & $-122.24(10)$ \\
\hline O1-S1-C13-C14 & $173.18(9)$ & O2-S1-C13-C14 & $-56.42(10)$ \\
\hline N2-S1-C13-C14 & $58.01(10)$ & C18-C13-C14-C15 & $0.72(18)$ \\
\hline
\end{tabular}




\begin{tabular}{|c|c|c|c|}
\hline S1-C13-C14-C15 & $-179.53(9)$ & C13-C14-C15-C16 & $-1.14(19)$ \\
\hline $\begin{array}{l}\text { C14-C15-C16- } \\
\text { C17 }\end{array}$ & $0.58(19)$ & C14-C15-C16-C25 & $-178.94(12)$ \\
\hline $\begin{array}{l}\text { C15-C16-C17- } \\
\text { C18 }\end{array}$ & $0.41(19)$ & C25-C16-C17-C18 & $179.93(12)$ \\
\hline $\begin{array}{l}\text { C14-C13-C18- } \\
\text { C17 }\end{array}$ & $0.24(18)$ & S1-C13-C18-C17 & $-179.51(9)$ \\
\hline $\begin{array}{l}\text { C16-C17-C18- } \\
\text { C13 }\end{array}$ & $-0.81(19)$ & O3-S2-C19-C20 & $158.15(9)$ \\
\hline O4-S2-C19-C20 & $28.85(10)$ & N1-S2-C19-C20 & $-85.95(10)$ \\
\hline O3-S2-C19-C24 & $-22.75(11)$ & O4-S2-C19-C24 & $-152.05(9)$ \\
\hline N1-S2-C19-C24 & $93.15(10)$ & C24-C19-C20-C21 & $0.57(17)$ \\
\hline S2-C19-C20-C21 & $179.66(8)$ & C19-C20-C21-C22 & $1.20(17)$ \\
\hline $\begin{array}{l}\text { C20-C21-C22- } \\
\text { C23 }\end{array}$ & $-1.72(17)$ & C20-C21-C22-C26 & $178.97(11)$ \\
\hline $\begin{array}{l}\text { C21-C22-C23- } \\
\text { C24 }\end{array}$ & $0.48(17)$ & $\mathrm{C} 26-\mathrm{C} 22-\mathrm{C} 23-\mathrm{C} 24$ & $179.79(11)$ \\
\hline $\begin{array}{l}\text { C22-C23-C24- } \\
\text { C19 }\end{array}$ & $1.24(17)$ & C20-C19-C24-C23 & $-1.78(17)$ \\
\hline $\mathrm{S} 2-\mathrm{C} 19-\mathrm{C} 24-\mathrm{C} 23$ & $179.13(9)$ & & \\
\hline
\end{tabular}

Table S7. Anisotropic atomic displacement parameters $\left(\AA^{2}\right)$ for MSU_032.

The anisotropic atomic displacement factor exponent takes the form: $-2 \pi^{2}\left[h^{2} a^{* 2}\right.$ $\left.U_{11}+\ldots+2 h k a^{*} b^{*} U_{12}\right]$ 

$\mathbf{U}_{11}$
$\mathbf{U}_{22}$
$\mathbf{U}_{33}$
$\mathbf{U}_{23}$
$\mathbf{U}_{13}$
$\mathrm{U}_{12}$

S1 $0.01432(13) 0.01382(12) 0.01397(13)-0.00320(10)-0.00038(9) \quad-$

$\begin{array}{lllllll}\mathrm{O} 1 & 0.0219(4) & 0.0224(4) & 0.0143(4) & -0.0008(3) & -0.0045(3) & -0.0107(3)\end{array}$

$\begin{array}{lllllll}\mathrm{O} 2 & 0.0189(4) & 0.0181(4) & 0.0264(4) & -0.0096(3) & 0.0024(3) & -0.0049(3)\end{array}$

S2 $0.01674(14) 0.02844(15) 0.01340(13)-0.00766(11) 0.00212(9) \quad$ o $0.01357(11)$

$\begin{array}{lllllll}\mathrm{O} 3 & 0.0267(5) & 0.0411(5) & 0.0252(4) & -0.0213(4) & 0.0079(4) & -0.0209(4)\end{array}$

$\begin{array}{lllllll}\mathrm{O} 4 & 0.0200(4) & 0.0381(5) & 0.0152(4) & -0.0009(4) & -0.0026(3) & -0.0150(4)\end{array}$

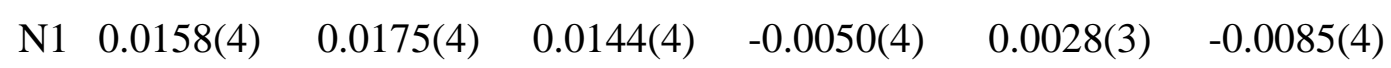

$\mathrm{N} 2 \quad 0.0187(5) \quad 0.0154(4) \quad 0.0117(4) \quad-0.0041(3) \quad 0.0018(3) \quad-0.0086(4)$

$\begin{array}{lllllll}\mathrm{C} 1 & 0.0136(5) & 0.0173(5) & 0.0189(5) & -0.0053(4) & 0.0000(4) & -0.0070(4)\end{array}$

$\begin{array}{lllllll}\mathrm{C} 2 & 0.0141(5) & 0.0145(5) & 0.0139(5) & -0.0042(4) & -0.0005(4) & -0.0062(4)\end{array}$

$\begin{array}{lllllll}\text { C3 } & 0.0144(5) & 0.0176(5) & 0.0130(5) & -0.0055(4) & -0.0007(4) & -0.0066(4)\end{array}$

$\begin{array}{lllllll}\mathrm{C} 4 & 0.0179(5) & 0.0179(5) & 0.0157(5) & -0.0071(4) & 0.0020(4) & -0.0072(4)\end{array}$

$\begin{array}{lllllll}\text { C5 } & 0.0191(5) & 0.0159(5) & 0.0170(5) & -0.0053(4) & 0.0024(4) & -0.0089(4)\end{array}$

$\begin{array}{lllllll}\mathrm{C} 6 & 0.0173(5) & 0.0200(5) & 0.0126(5) & -0.0050(4) & -0.0005(4) & -0.0089(4)\end{array}$

$\begin{array}{lllllll}\text { C7 } & 0.0211(6) & 0.0213(5) & 0.0162(5) & -0.0033(4) & -0.0002(4) & -0.0087(5)\end{array}$

$\begin{array}{lllllll}\mathrm{C} 8 & 0.0275(6) & 0.0272(6) & 0.0158(5) & -0.0003(5) & -0.0018(4) & -0.0153(5)\end{array}$

$\begin{array}{lllllll}\text { C9 } & 0.0263(6) & 0.0390(7) & 0.0138(5) & -0.0073(5) & 0.0032(4) & -0.0198(6)\end{array}$

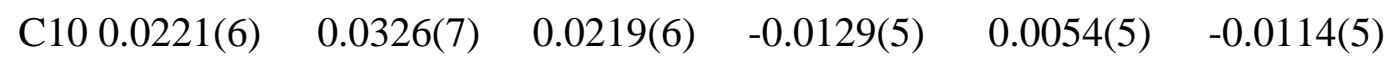

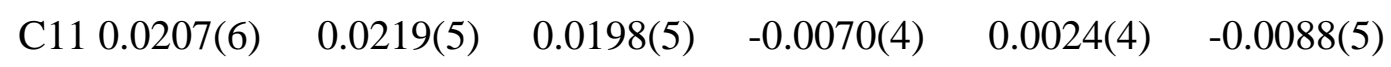

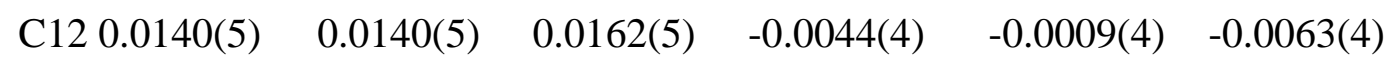

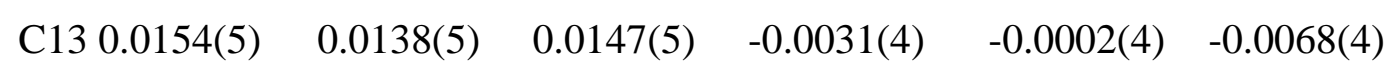

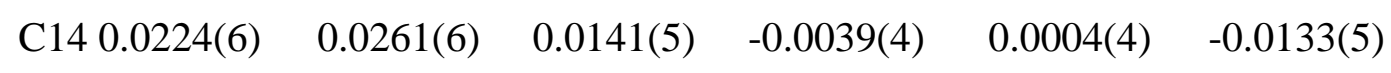




$\begin{array}{cccccc}\text { U11 } & \mathbf{U}_{22} & \mathbf{U}_{33} & \mathbf{U}_{23} & \mathbf{U}_{13} & \mathbf{U}_{12} \\ \text { C15 0.0225(6) } & 0.0285(6) & 0.0196(6) & -0.0058(5) & -0.0036(4) & -0.0125(5) \\ \text { C16 0.0178(6) } & 0.0228(6) & 0.0261(6) & -0.0062(5) & 0.0005(4) & -0.0100(5) \\ \text { C17 0.0219(6) } & 0.0300(6) & 0.0188(5) & -0.0043(5) & 0.0048(4) & -0.0138(5) \\ \text { C18 0.0210(6) } & 0.0231(5) & 0.0140(5) & -0.0029(4) & -0.0001(4) & -0.0104(5) \\ \text { C19 0.0162(5) } & 0.0251(5) & 0.0109(5) & -0.0060(4) & 0.0022(4) & -0.0119(4) \\ \text { C20 0.0177(5) } & 0.0230(5) & 0.0150(5) & -0.0055(4) & 0.0013(4) & -0.0086(4) \\ \text { C21 0.0232(6) } & 0.0227(5) & 0.0177(5) & -0.0057(4) & 0.0010(4) & -0.0131(5) \\ \text { C22 0.0197(6) } & 0.0286(6) & 0.0129(5) & -0.0043(4) & 0.0004(4) & -0.0144(5) \\ \text { C23 0.0159(5) } & 0.0266(6) & 0.0182(5) & -0.0074(5) & 0.0009(4) & -0.0083(5) \\ \text { C24 0.0204(6) } & 0.0227(5) & 0.0169(5) & -0.0076(4) & 0.0019(4) & -0.0104(5) \\ \text { C25 0.0207(6) } & 0.0461(8) & 0.0390(8) & -0.0089(7) & 0.0002(5) & -0.0180(6) \\ \text { C26 0.0234(6) } & 0.0353(7) & 0.0333(7) & -0.0036(6) & -0.0055(5) & -0.0186(6)\end{array}$

Table S8. Hydrogen atomic coordinates and isotropic atomic displacement parameters $\left(\AA^{2}\right)$ for $M S U \_032$.

\begin{tabular}{ccccc}
\hline & $\mathbf{x} / \mathbf{a}$ & $\mathbf{y} / \mathbf{b}$ & $\mathbf{z} / \mathbf{c}$ & $\mathbf{U}(\mathbf{e q})$ \\
$\mathrm{H} 1 \mathrm{~A}$ & 0.6748 & 0.4872 & 0.3000 & 0.019 \\
$\mathrm{H} 1 \mathrm{~B}$ & 0.6676 & 0.4045 & 0.2166 & 0.019 \\
$\mathrm{H} 2$ & 0.5405 & 0.3458 & 0.3786 & 0.017 \\
$\mathrm{H} 4$ & 0.3230 & 0.7579 & 0.3603 & 0.02 \\
$\mathrm{H} 5 \mathrm{~A}$ & 0.3978 & 0.8205 & 0.1820 & 0.02 \\
$\mathrm{H} 5 \mathrm{~B}$ & 0.5413 & 0.7617 & 0.2521 & 0.02
\end{tabular}




\begin{tabular}{|c|c|c|c|c|}
\hline & $\mathbf{x} / \mathbf{a}$ & $\mathbf{y} / \mathbf{b}$ & $\mathbf{z} / \mathbf{c}$ & $\mathbf{U}(\mathbf{e q})$ \\
\hline $\mathrm{H} 7$ & 0.4018 & 0.2950 & 0.4924 & 0.024 \\
\hline H8 & 0.2501 & 0.2368 & 0.6288 & 0.028 \\
\hline H9 & 0.0491 & 0.4185 & 0.6766 & 0.029 \\
\hline H10 & -0.0002 & 0.6600 & 0.5864 & 0.029 \\
\hline H11 & 0.1488 & 0.7192 & 0.4488 & 0.024 \\
\hline $\mathrm{H} 12 \mathrm{~A}$ & 0.3271 & 0.3663 & 0.2951 & 0.017 \\
\hline H12B & 0.3654 & 0.4689 & 0.1917 & 0.017 \\
\hline H14 & 0.3232 & 0.1374 & 0.1346 & 0.024 \\
\hline H15 & 0.0900 & 0.1331 & 0.1359 & 0.027 \\
\hline H17 & 0.0848 & 0.0402 & 0.4514 & 0.028 \\
\hline H18 & 0.3154 & 0.0505 & 0.4515 & 0.023 \\
\hline $\mathrm{H} 20$ & 0.6483 & 0.9051 & 0.0703 & 0.022 \\
\hline $\mathrm{H} 21$ & 0.8540 & 0.9523 & 0.0918 & 0.024 \\
\hline $\mathrm{H} 23$ & 1.1121 & 0.5355 & 0.1101 & 0.024 \\
\hline $\mathrm{H} 24$ & 0.9071 & 0.4845 & 0.0947 & 0.023 \\
\hline $\mathrm{H} 25 \mathrm{~A}$ & -0.0774 & -0.0216 & 0.3122 & 0.051 \\
\hline $\mathrm{H} 25 \mathrm{~B}$ & -0.1321 & 0.1301 & 0.2261 & 0.051 \\
\hline $\mathrm{H} 25 \mathrm{C}$ & -0.1476 & 0.1252 & 0.3478 & 0.051 \\
\hline $\mathrm{H} 26 \mathrm{~A}$ & 1.1111 & 0.8856 & 0.0939 & 0.043 \\
\hline $\mathrm{H} 26 \mathrm{~B}$ & 1.2137 & 0.7384 & 0.0659 & 0.043 \\
\hline $\mathrm{H} 26 \mathrm{C}$ & 1.1713 & 0.7396 & 0.1849 & 0.043 \\
\hline HN2 & $0.5061(19)$ & $0.2653(19)$ & $0.1505(14)$ & $0.033(4)$ \\
\hline
\end{tabular}




\section{X-ray Crystallography for 11}

A colorless crystal prism like of $\mathrm{C}_{32} \mathrm{H}_{32} \mathrm{~N}_{2} \mathrm{O}_{4} \mathrm{~S}_{2}$, approximate dimensions $(0.177 \mathrm{x}$ $0.272 \times 0.421) \mathrm{mm}^{3}$, was selected for the X-ray crystallographic analysis and mounted on a cryoloop using an oil cryoprotectant. The X-ray intensity data was measured at low temperature $(\mathrm{T}=100 \mathrm{~K})$, using a three circles goniometer Kappa geometry with a fixed Kappa angle at $=54.74$ deg Bruker AXS D8 Venture, equipped with a shutterless Photon 100 CMOS active pixel sensor detector. Frames were integrated with the Bruker SAINT software ${ }^{6}$ using a narrow-frame algorithm. The integration of the data using a monoclinic unit cell yielded a total of 50601 reflections to a maximum $\theta$ angle of $72.52^{\circ}(0.81 \AA$ resolution), of which 5791 were independent (average redundancy 8.738 , completeness $=99.5 \%, \mathrm{R}_{\mathrm{int}}=2.29 \%, \mathrm{R}_{\mathrm{ig}}=1.43 \%$ ) and 5623 $(97.10 \%)$ were greater than $2 \sigma\left(F^{2}\right)$. The final cell constants of $\underline{a}=10.9602(4) \AA, \underline{b}=$ 14.7542(6) $\AA, \underline{c}=18.1626(7) \AA, \beta=94.8570(10)^{\circ}$, volume $=2926.5(2) \AA^{3}$, are based upon the refinement of the XYZ-centroids of 8454 reflections above $20 \sigma$ (I) with $8.082^{\circ}<2 \theta<51.43^{\circ}$. Data were corrected for absorption effects using the Multi-Scan method implemented in the program (SADABS) ${ }^{7}$. The ratio of minimum to maximum apparent transmission was 0.834 . The calculated minimum and maximum transmission coefficients (based on crystal size) are 0.4910 and 0.7220 . The structure was solved in the monoclinic unit cell using the Bruker SHELXTL Software Package ${ }^{8}$, using the space group P $12(1) / c 1$, with $Z=4$ for the formula unit, C32H32N2O4S2. Refinement of the structure was carried out by least squares procedures on weighted $\mathrm{F}^{2}$ values using the SHELXL-2016/6 (Sheldrick, 2016) ${ }^{9}$ included in the APEX3 v2018, 1.0, AXS Bruker program ${ }^{10}$. Hydrogen atoms were localized on difference Fourier maps but then introduced in the refinement as fixed contributors in idealized geometry with an isotropic thermal parameters fixed at $20 \%$ higher than those carbons atoms they were connected. The final anisotropic full-matrix least-squares 
refinement on $\mathrm{F}^{2}$ with 364 variables converged at $\mathrm{R} 1=3.28 \%$, for the observed data and $\mathrm{wR} 2=9.98 \%$ for all data. The goodness-of-fit: GOF was 1.094 . The largest peak in the final difference electron density synthesis was $0.438 \mathrm{e}^{-} / \AA^{3}$ and the largest hole was $-0.364 \mathrm{e}^{-} / \AA^{3}$ with an RMS deviation of $0.085 \mathrm{e}^{-} / \AA^{3}$. Based on the final model, the calculated density was $1.300 \mathrm{~g} / \mathrm{cm}^{3}$ and $\mathrm{F}(000), 1208 \mathrm{e}^{-}$.
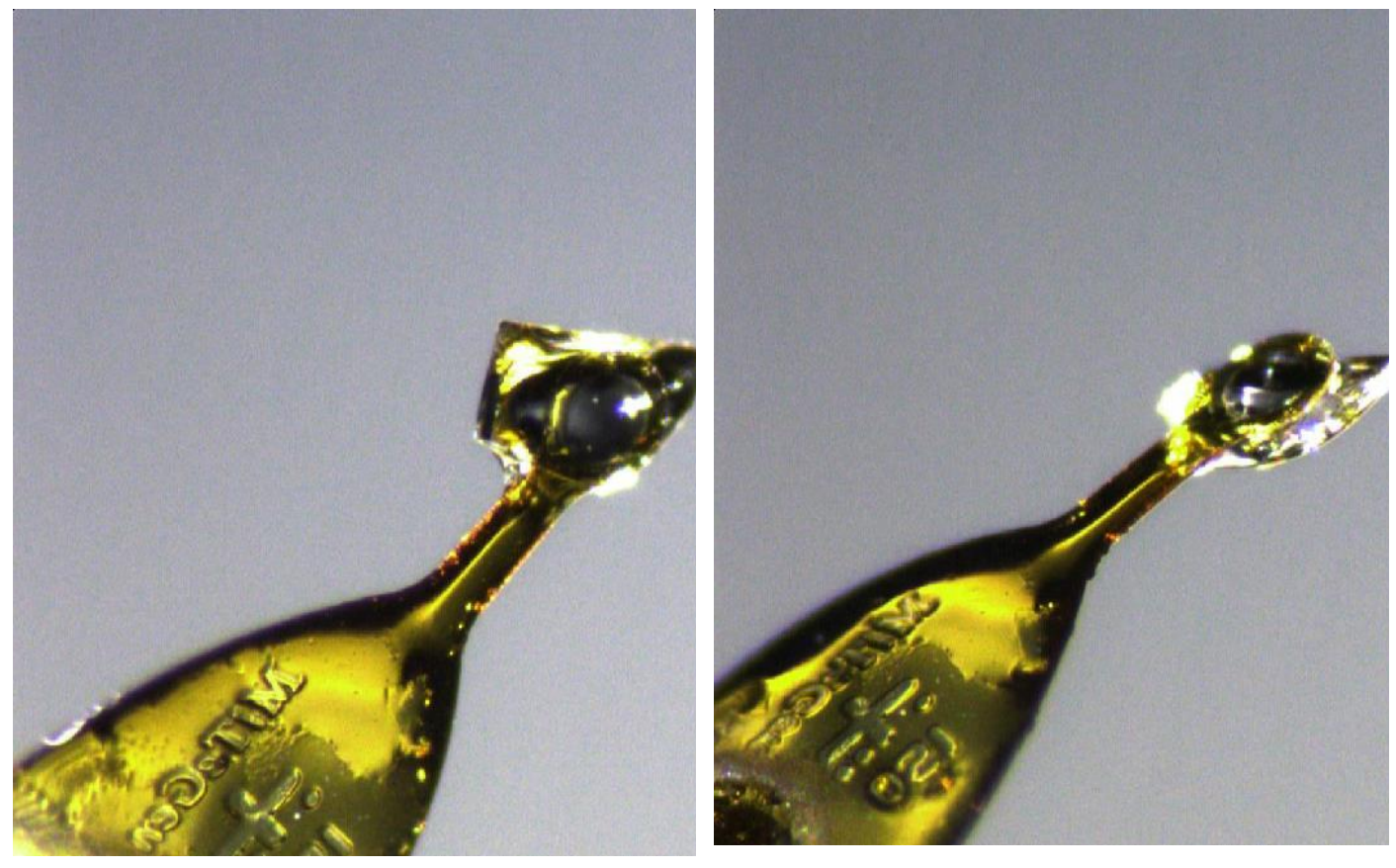

Crystal's view: 

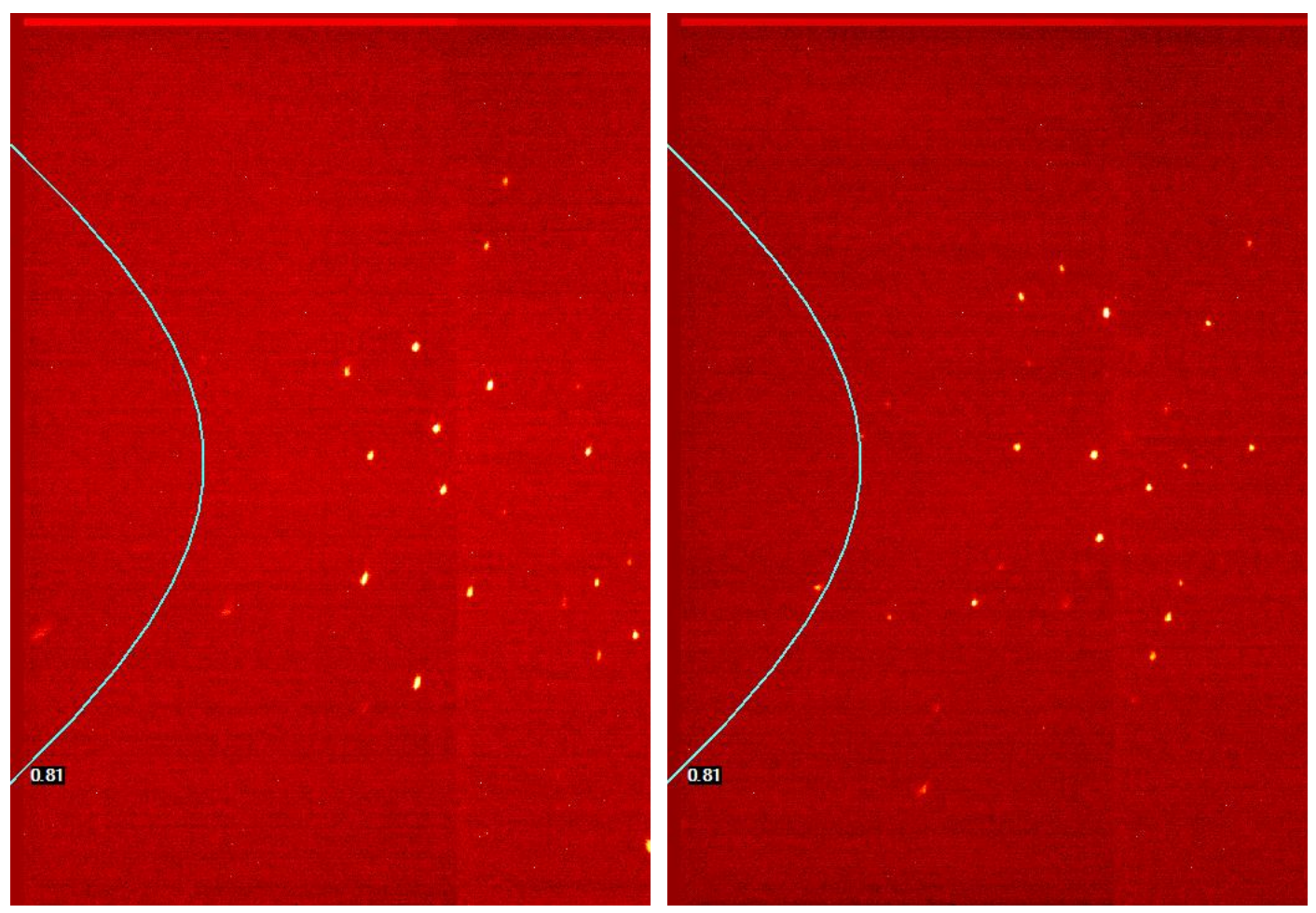

Crystal's Resolution: using a Cu monochromatized $X$-ray radiation : $\lambda=1.54178$ $\AA$ 


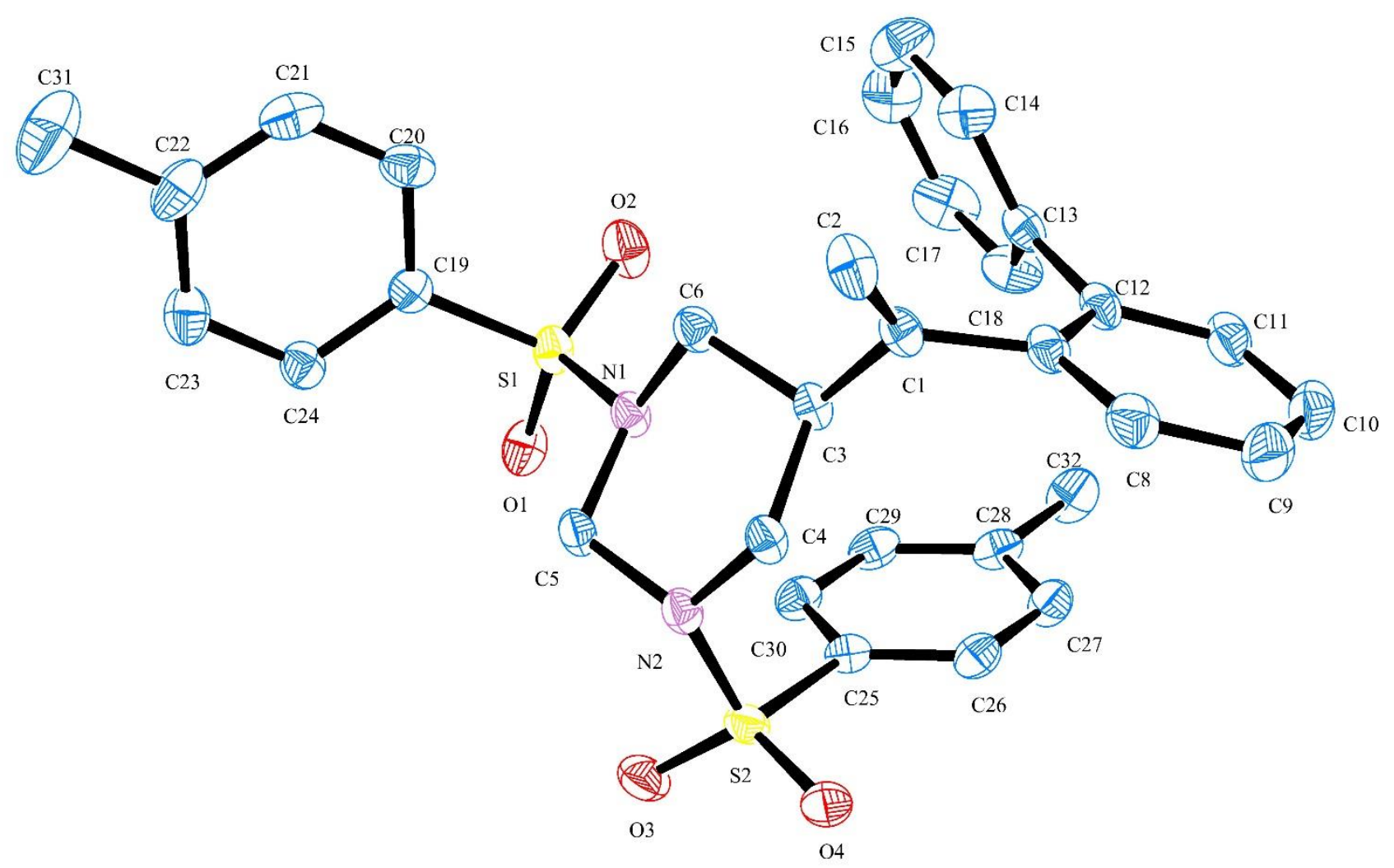

Asymmetric unit's view:

Table S9. Sample and crystal data for i2791_1

Identification code $\quad$ i2791_1

Chemical formula $\quad \mathrm{C}_{32} \mathrm{H}_{32} \mathrm{~N}_{2} \mathrm{O}_{4} \mathrm{~S}_{2}$

Formula weight $\quad 572.71 \mathrm{~g} / \mathrm{mol}$

$\begin{array}{ll}\text { Temperature } & \text { 100(2) K }\end{array}$

Wavelength $\quad 1.54178 \AA$

Crystal size $\quad(0.177 \times 0.272 \times 0.42) 1 \mathrm{~mm}^{3}$

Crystal system monoclinic 
Space group

P $12(1) / c 1$

Unit cell dimensions

$$
\begin{aligned}
& \mathrm{a}=10.9602(4) \AA \quad \alpha=90^{\circ} \\
& \mathrm{b}=14.7542(6) \AA \quad \beta=94.8570(10)^{\circ} \\
& \mathrm{c}=18.1626(7) \AA \quad \gamma=90^{\circ}
\end{aligned}
$$

Volume

$2926.5(2) \AA^{3}$

$\mathbf{Z}$

4

Density (calculated) $\quad 1.300 \mathrm{~g} / \mathrm{cm}^{3}$

Absorption coefficient $1.968 \mathrm{~mm}^{-1}$

$\mathbf{F}(\mathbf{0 0 0}) \quad 1208$

Table S10. Data collection and structure refinement for i2791_1

$\begin{aligned} & \text { Theta range for data } \\ & \text { collection }\end{aligned}$
$\begin{array}{ll}\text { Index ranges } & -13<=\mathrm{h}<=13,-18<=\mathrm{k}<=16,- \\ 22<=\mathrm{l}<=22\end{array}$

Reflections collected 50601

Independent

reflections

$5791[\mathrm{R}(\mathrm{int})=0.0229]$

Coverage of

independent $\quad 99.5 \%$

reflections

Absorption

correction

Multi-Scan

Max. and min.

transmission

0.7220 and 0.4910 


\begin{tabular}{|c|c|c|}
\hline $\begin{array}{l}\text { Structure solution } \\
\text { technique }\end{array}$ & \multicolumn{2}{|c|}{ direct methods } \\
\hline $\begin{array}{l}\text { Structure solution } \\
\text { program }\end{array}$ & \multicolumn{2}{|c|}{ XT, VERSION 2014/5 } \\
\hline Refinement method & \multicolumn{2}{|c|}{ Full-matrix least-squares on $\mathrm{F}^{2}$} \\
\hline $\begin{array}{l}\text { Refinement } \\
\text { program }\end{array}$ & \multicolumn{2}{|c|}{ SHELXL-2017/1 (Sheldrick, 2017) } \\
\hline Function minimized & \multicolumn{2}{|c|}{$\Sigma \mathrm{w}\left(\mathrm{F}_{\mathrm{o}}^{2}-\mathrm{F}_{\mathrm{c}}^{2}\right)^{2}$} \\
\hline $\begin{array}{l}\text { Data / restraints / } \\
\text { parameters }\end{array}$ & \multicolumn{2}{|c|}{$5791 / 0$ / 364} \\
\hline $\begin{array}{l}\text { Goodness-of-fit on } \\
\text { F }^{2}\end{array}$ & \multicolumn{2}{|l|}{1.094} \\
\hline \multirow[t]{2}{*}{ Final $\mathbf{R}$ indices } & $\begin{array}{l}5623 \text { data; } \\
\mathrm{I}>2 \sigma(\mathrm{I})\end{array}$ & $\begin{array}{l}\mathrm{R} 1=0.0328, \mathrm{wR} 2= \\
0.0933\end{array}$ \\
\hline & all data & $\begin{array}{l}\mathrm{R} 1=0.0344, \mathrm{wR} 2= \\
0.0998\end{array}$ \\
\hline Weighting scheme & \multicolumn{2}{|c|}{$\begin{array}{l}\mathrm{w}=1 /\left[\sigma^{2}\left(\mathrm{~F}_{\mathrm{o}}^{2}\right)+(0.0531 \mathrm{P})^{2}+1.4839 \mathrm{P}\right] \\
\text { where } \mathrm{P}=\left(\mathrm{F}_{\mathrm{o}}^{2}+2 \mathrm{~F}_{\mathrm{c}}^{2}\right) / 3\end{array}$} \\
\hline $\begin{array}{l}\text { Extinction } \\
\text { coefficient }\end{array}$ & \multicolumn{2}{|l|}{$0.0008(1)$} \\
\hline $\begin{array}{l}\text { Largest diff. peak } \\
\text { and hole }\end{array}$ & \multicolumn{2}{|c|}{0.438 and $-0.364 \mathrm{e}^{-3}$} \\
\hline $\begin{array}{l}\text { R.M.S. deviation } \\
\text { from mean }\end{array}$ & \multicolumn{2}{|l|}{$0.085 \mathrm{e}^{-3}$} \\
\hline
\end{tabular}

Table S11. Atomic coordinates and equivalent isotropic atomic displacement parameters $\left(\AA^{2}\right)$ for $12791 \_1$.

$U(e q)$ is defined as one third of the trace of the orthogonalized $U_{i j}$ tensor. 
$\mathbf{x} / \mathbf{a}$

$\mathrm{S}$

S1

S2

$\mathrm{N}$

O1

$\mathrm{O} 2$

$\mathrm{O} 3$

$\mathrm{O} 4$

$\mathrm{C}$

C2 $0.82341(14)$

C3 0.69574(12)

C4 0.62978(12)

C5 $\quad 0.43318(12) \quad 0.28609(10)$

C6 $0.61055(12) \quad 0.23995(10)$

C7 $\quad 0.92470(12) \quad 0.28140(10)$

C8 $0.98722(14) \quad 0.27646(11)$

C9 $\quad 0.07962(15) \quad 0.33813(13)$

C10 0.10971(15) 0.40515(11)

$\begin{array}{lll}\mathrm{C} 11 & 0.05047(14) & 0.41014(10)\end{array}$

C12 0.95895(12) $\quad 0.34844(10)$

$\begin{array}{lll}\mathrm{C} 13 & 0.90202(13) & 0.35382(10)\end{array}$

C14 0.90063(16) $\quad 0.28002(11)$

C15 0.84983(19) $\mathbf{z} / \mathbf{c}$

$0.42323(2)$

$0.18799(2)$

$0.35218(6)$

$0.22180(6)$

$0.40136(6)$

$0.48554(6)$

$0.18353(6)$

$0.12297(6)$

$0.30550(8)$

0.30431(9)

$0.30477(7)$

$0.22660(7)$

$0.27920(8)$

$0.36163(8)$

0.29796(8)

$0.23436(9)$

$0.22184(10)$

$0.27313(11)$

$0.33719(10)$

$0.35151(8)$

$0.42300(8)$

0.47053(9)

$0.53759(10)$
$\mathrm{U}(\mathrm{eq})$

$0.02226(11)$

0.02233(11)

0.0209(2)

$0.0210(2)$

$0.0288(2)$

0.0327(3)

0.0308(3)

0.0283(2)

0.0219(3)

$0.0300(3)$

0.0192(3)

0.0208(3)

0.0220(3)

0.0221(3)

$0.0220(3)$

0.0296(3)

0.0363(4)

0.0352(4)

0.0292(3)

0.0226(3)

0.0232(3)

0.0312(3)

0.0387(4) 


\begin{tabular}{|c|c|c|c|c|}
\hline & $\mathbf{x} / \mathbf{a}$ & $\mathbf{y} / \mathbf{b}$ & $\mathbf{z} / \mathbf{c}$ & $\mathbf{U}(\mathbf{e q})$ \\
\hline $\mathrm{C} 16$ & $0.79609(18)$ & $0.36759(13)$ & $0.55760(9)$ & $0.0396(4)$ \\
\hline $\mathrm{C} 17$ & $0.79710(18)$ & $0.44146(13)$ & $0.51100(10)$ & $0.0400(4)$ \\
\hline $\mathrm{C} 18$ & $0.85098(16)$ & $0.43500(12)$ & $0.44483(9)$ & $0.0331(4)$ \\
\hline C19 & $0.34025(13)$ & $0.21200(10)$ & $0.43677(7)$ & $0.0213(3)$ \\
\hline $\mathrm{C} 20$ & $0.39742(14)$ & $0.14487(11)$ & $0.48093(8)$ & $0.0285(3)$ \\
\hline $\mathrm{C} 21$ & $0.33631(16)$ & $0.06453(12)$ & $0.49055(9)$ & $0.0336(4)$ \\
\hline $\mathrm{C} 22$ & $0.21913(16)$ & $0.05007(11)$ & $0.45737(10)$ & $0.0334(4)$ \\
\hline $\mathrm{C} 23$ & $0.16327(14)$ & $0.11850(11)$ & $0.41392(9)$ & $0.0310(3)$ \\
\hline $\mathrm{C} 24$ & $0.22281(13)$ & $0.19978(10)$ & $0.40295(8)$ & $0.0246(3)$ \\
\hline $\mathrm{C} 25$ & $0.57801(13)$ & $0.49300(10)$ & $0.25028(8)$ & $0.0220(3)$ \\
\hline $\mathrm{C} 26$ & $0.69495(14)$ & $0.52198(10)$ & $0.23748(8)$ & $0.0252(3)$ \\
\hline $\mathrm{C} 27$ & $0.75545(15)$ & $0.58220(10)$ & $0.28648(8)$ & $0.0275(3)$ \\
\hline $\mathrm{C} 28$ & $0.70149(15)$ & $0.61304(10)$ & $0.34880(8)$ & $0.0261(3)$ \\
\hline $\mathrm{C} 29$ & $0.58431(15)$ & $0.58244(10)$ & $0.36055(8)$ & $0.0268(3)$ \\
\hline $\mathrm{C} 30$ & $0.52201(14)$ & $0.52312(10)$ & $0.31206(8)$ & $0.0253(3)$ \\
\hline C31 & $0.1568(2)$ & $0.96033(14)$ & $0.46721(15)$ & $0.0576(6)$ \\
\hline C32 & $0.76801(18)$ & $0.67735(12)$ & $0.40262(9)$ & $0.0365(4)$ \\
\hline
\end{tabular}

Table S12. Bond lengths (Å) for i2791_1.

\begin{tabular}{|c|c|c|c|}
\hline $\mathrm{S} 1-\mathrm{O} 2$ & $1.4289(11)$ & S1-O1 & $1.4321(11)$ \\
\hline S1-N1 & $1.6374(11)$ & S1-C19 & $1.7579(15)$ \\
\hline $\mathrm{S} 2-\mathrm{O} 3$ & $1.4337(11)$ & S2-O4 & $1.4340(11)$ \\
\hline
\end{tabular}




\begin{tabular}{|c|c|c|c|}
\hline S2-N2 & $1.6427(13)$ & S2-C25 & $1.7633(15)$ \\
\hline N1-C5 & $1.4687(17)$ & N1-C6 & $1.4723(18)$ \\
\hline $\mathrm{N} 2-\mathrm{C} 5$ & $1.4557(17)$ & $\mathrm{N} 2-\mathrm{C} 4$ & $1.4714(17)$ \\
\hline $\mathrm{C} 1-\mathrm{C} 2$ & $1.333(2)$ & $\mathrm{C} 1-\mathrm{C} 7$ & $1.4902(19)$ \\
\hline $\mathrm{C} 1-\mathrm{C} 3$ & $1.5219(18)$ & $\mathrm{C} 2-\mathrm{H} 2 \mathrm{~A}$ & 0.95 \\
\hline C2-H2B & 0.95 & C3-C6 & $1.5279(19)$ \\
\hline $\mathrm{C} 3-\mathrm{C} 4$ & $1.5400(18)$ & C3-H3 & 1.0 \\
\hline C4-H4A & 0.99 & C4-H4B & 0.99 \\
\hline C5-H5A & 0.99 & C5-H5B & 0.99 \\
\hline C6-H6A & 0.99 & C6-H6B & 0.99 \\
\hline C7-C8 & $1.394(2)$ & C7-C12 & $1.415(2)$ \\
\hline $\mathrm{C} 8-\mathrm{C} 9$ & $1.394(2)$ & C8-H8 & 0.95 \\
\hline C9-C10 & $1.379(3)$ & C9-H9 & 0.95 \\
\hline C10-C11 & $1.382(2)$ & C10-H10 & 0.95 \\
\hline C11-C12 & $1.395(2)$ & C11-H11 & 0.95 \\
\hline C12-C13 & $1.489(2)$ & C13-C14 & $1.391(2)$ \\
\hline C13-C18 & $1.394(2)$ & C14-C15 & $1.386(2)$ \\
\hline C14-H14 & 0.95 & C15-C16 & $1.383(3)$ \\
\hline C15-H15 & 0.95 & C16-C17 & $1.381(3)$ \\
\hline C16-H16 & 0.95 & C17-C18 & $1.387(2)$ \\
\hline C17-H17 & 0.95 & C18-H18 & 0.95 \\
\hline C19-C20 & $1.390(2)$ & C19-C24 & $1.390(2)$ \\
\hline C20-C21 & $1.380(2)$ & $\mathrm{C} 20-\mathrm{H} 20$ & 0.95 \\
\hline $\mathrm{C} 21-\mathrm{C} 22$ & $1.388(3)$ & $\mathrm{C} 21-\mathrm{H} 21$ & 0.95 \\
\hline C22-C23 & $1.391(2)$ & C22-C31 & $1.507(2)$ \\
\hline
\end{tabular}




$\begin{array}{llll}\mathrm{C} 23-\mathrm{C} 24 & 1.388(2) & \mathrm{C} 23-\mathrm{H} 23 & 0.95 \\ \mathrm{C} 24-\mathrm{H} 24 & 0.95 & \mathrm{C} 25-\mathrm{C} 26 & 1.389(2) \\ \mathrm{C} 25-\mathrm{C} 30 & 1.396(2) & \mathrm{C} 26-\mathrm{C} 27 & 1.386(2) \\ \mathrm{C} 26-\mathrm{H} 26 & 0.95 & \mathrm{C} 27-\mathrm{C} 28 & 1.397(2) \\ \mathrm{C} 27-\mathrm{H} 27 & 0.95 & \mathrm{C} 28-\mathrm{C} 29 & 1.395(2) \\ \mathrm{C} 28-\mathrm{C} 32 & 1.506(2) & \mathrm{C} 29-\mathrm{C} 30 & 1.380(2) \\ \mathrm{C} 29-\mathrm{H} 29 & 0.95 & \mathrm{C} 30-\mathrm{H} 30 & 0.95 \\ \mathrm{C} 31-\mathrm{H} 31 \mathrm{~A} & 0.98 & \mathrm{C} 31-\mathrm{H} 31 \mathrm{~B} & 0.98 \\ \mathrm{C} 31-\mathrm{H} 31 \mathrm{C} & 0.98 & \mathrm{C} 32-\mathrm{H} 32 \mathrm{~A} & 0.98 \\ \mathrm{C} 32-\mathrm{H} 32 \mathrm{~B} & 0.98 & \mathrm{C} 32-\mathrm{H} 32 \mathrm{C} & 0.98\end{array}$

Table S13. Bond angles $\left(^{\circ}\right)$ for i2791_1.

\begin{tabular}{llll}
\hline O2-S1-O1 & $120.55(7)$ & O2-S1-N1 & $106.24(6)$ \\
O1-S1-N1 & $106.07(6)$ & O2-S1-C19 & $108.25(7)$ \\
O1-S1-C19 & $107.96(7)$ & N1-S1-C19 & $107.06(6)$ \\
O3-S2-O4 & $120.78(6)$ & O3-S2-N2 & $105.84(7)$ \\
O4-S2-N2 & $105.70(6)$ & O3-S2-C25 & $107.52(7)$ \\
O4-S2-C25 & $106.90(7)$ & N2-S2-C25 & $109.84(6)$ \\
C5-N1-C6 & $112.67(11)$ & C5-N1-S1 & $117.86(9)$ \\
C6-N1-S1 & $118.73(9)$ & C5-N2-C4 & $113.39(11)$ \\
C5-N2-S2 & $119.92(10)$ & C4-N2-S2 & $119.11(9)$
\end{tabular}




\begin{tabular}{|c|c|c|c|}
\hline $\mathrm{C} 2-\mathrm{C} 1-\mathrm{C} 7$ & $122.81(13)$ & $\mathrm{C} 2-\mathrm{C} 1-\mathrm{C} 3$ & $122.75(13)$ \\
\hline $\mathrm{C} 7-\mathrm{C} 1-\mathrm{C} 3$ & 114.01(12) & $\mathrm{C} 1-\mathrm{C} 2-\mathrm{H} 2 \mathrm{~A}$ & 120.0 \\
\hline $\mathrm{C} 1-\mathrm{C} 2-\mathrm{H} 2 \mathrm{~B}$ & 120.0 & $\mathrm{H} 2 \mathrm{~A}-\mathrm{C} 2-\mathrm{H} 2 \mathrm{~B}$ & 120.0 \\
\hline C1-C3-C6 & $114.86(12)$ & $\mathrm{C} 1-\mathrm{C} 3-\mathrm{C} 4$ & $108.72(11)$ \\
\hline C6-C3-C4 & $109.85(11)$ & C1-C3-H3 & 107.7 \\
\hline C6-C3-H3 & 107.7 & C4-C3-H3 & 107.7 \\
\hline $\mathrm{N} 2-\mathrm{C} 4-\mathrm{C} 3$ & 111.93(11) & $\mathrm{N} 2-\mathrm{C} 4-\mathrm{H} 4 \mathrm{~A}$ & 109.2 \\
\hline C3-C4-H4A & 109.2 & $\mathrm{~N} 2-\mathrm{C} 4-\mathrm{H} 4 \mathrm{~B}$ & 109.2 \\
\hline C3-C4-H4B & 109.2 & H4A-C4-H4B & 107.9 \\
\hline $\mathrm{N} 2-\mathrm{C} 5-\mathrm{N} 1$ & 109.81(11) & N2-C5-H5A & 109.7 \\
\hline N1-C5-H5A & 109.7 & N2-C5-H5B & 109.7 \\
\hline N1-C5-H5B & 109.7 & H5A-C5-H5B & 108.2 \\
\hline N1-C6-C3 & $106.50(11)$ & N1-C6-H6A & 110.4 \\
\hline C3-C6-H6A & 110.4 & N1-C6-H6B & 110.4 \\
\hline С3-С6-H6B & 110.4 & H6A-C6-H6B & 108.6 \\
\hline C8-C7-C12 & $119.05(14)$ & $\mathrm{C} 8-\mathrm{C} 7-\mathrm{C} 1$ & $119.70(13)$ \\
\hline C12-C7-C1 & $121.04(13)$ & C7-C8-C9 & $121.05(15)$ \\
\hline C7-C8-H8 & 119.5 & C9-C8-H8 & 119.5 \\
\hline C10-C9-C8 & $119.55(15)$ & C10-C9-H9 & 120.2 \\
\hline C8-C9-H9 & 120.2 & C9-C10-C11 & $120.30(15)$ \\
\hline C9-C10-H10 & 119.9 & C11-C10-H10 & 119.9 \\
\hline C10-C11-C12 & $121.25(15)$ & C10-C11-H11 & 119.4 \\
\hline C12-C11-H11 & 119.4 & C11-C12-C7 & $118.74(14)$ \\
\hline C11-C12-C13 & $119.25(13)$ & C7-C12-C13 & $122.00(13)$ \\
\hline C14-C13-C18 & $118.07(14)$ & C14-C13-C12 & $121.79(13)$ \\
\hline
\end{tabular}




$\begin{array}{llll}\text { C18-C13-C12 } & 120.11(14) & \text { C15-C14-C13 } & 120.65(15) \\ \text { C15-C14-H14 } & 119.7 & \text { C13-C14-H14 } & 119.7 \\ \text { C16-C15-C14 } & 120.70(16) & \text { C16-C15-H15 } & 119.6 \\ \text { C14-C15-H15 } & 119.6 & \text { C17-C16-C15 } & 119.21(16) \\ \text { C17-C16-H16 } & 120.4 & \text { C15-C16-H16 } & 120.4 \\ \text { C16-C17-C18 } & 120.21(16) & \text { C16-C17-H17 } & 119.9 \\ \text { C18-C17-H17 } & 119.9 & \text { C17-C18-C13 } & 121.09(16) \\ \text { C17-C18-H18 } & 119.5 & \text { C13-C18-H18 } & 119.5 \\ \text { C20-C19-C24 } & 121.19(14) & \text { C20-C19-S1 } & 118.68(11) \\ \text { C24-C19-S1 } & 120.13(11) & \text { C21-C20-C19 } & 119.03(15) \\ \text { C21-C20-H20 } & 120.5 & \text { C19-C20-H20 } & 120.5 \\ \text { C20-C21-C22 } & 121.21(15) & \text { C20-C21-H21 } & 119.4 \\ \text { C22-C21-H21 } & 119.4 & \text { C21-C22-C23 } & 118.77(15) \\ \text { C21-C22-C31 } & 119.70(17) & \text { C23-C22-C31 } & 121.51(18) \\ \text { C24-C23-C22 } & 121.26(15) & \text { C24-C23-H23 } & 119.4 \\ \text { C22-C23-H23 } & 119.4 & \text { C23-C24-C19 } & 118.54(14) \\ \text { C23-C24-H24 } & 120.7 & \text { C19-C24-H24 } & 120.7 \\ \text { C26-C25-C30 } & 120.73(13) & \text { C26-C25-S2 } & 119.37(11) \\ \text { C30-C25-S2 } & 119.90(11) & \text { C27-C26-C25 } & 119.20(13) \\ \text { C27-C26-H26 } & 120.4 & \text { C25-C26-H26 } & 120.4 \\ \text { C26-C27-C28 } & 121.11(14) & \text { C26-C27-H27 } & 119.4 \\ \text { C28-C27-H27 } & 119.4 & \text { C29-C28-C27 } & 118.49(14) \\ \text { C29-C28-C32 } & 120.42(14) & \text { C27-C28-C32 } & 121.09(15) \\ \text { C30-C29-C28 } & 121.32(14) & 119.3 & 119.3 \\ \text { C28-C29-H29 } & 119-C 25 & 119.14(14)\end{array}$




$\begin{array}{lllr}\text { C29-C30-H30 } & 120.4 & \text { C25-C30-H30 } & 120.4 \\ \text { C22-C31-H31A } & 109.5 & \text { C22-C31-H31B } & 109.5 \\ \text { H31A-C31-H31B 109.5 } & \text { C22-C31-H31C } & 109.5 \\ \text { H31A-C31-H31C 109.5 } & \text { H31B-C31-H31C } & 109.5 \\ \text { C28-C32-H32A } & 109.5 & \text { C28-C32-H32B } & 109.5 \\ \text { H32A-C32-H32B 109.5 } & \text { C28-C32-H32C } & 109.5 \\ \text { H32A-C32-H32C 109.5 } & \text { H32B-C32-H32C } & 109.5\end{array}$

Table S14. Torsion angles $\left({ }^{\circ}\right)$ for i2791_1.

\begin{tabular}{llll}
\hline & & & \\
\hline O2-S1-N1-C5 & $-176.81(11)$ & O1-S1-N1-C5 & $-47.40(12)$ \\
C19-S1-N1-C5 & $67.69(12)$ & O2-S1-N1-C6 & $41.31(12)$ \\
O1-S1-N1-C6 & $170.71(10)$ & C19-S1-N1-C6 & $-74.20(11)$ \\
O3-S2-N2-C5 & $-38.10(12)$ & O4-S2-N2-C5 & $-167.33(10)$ \\
C25-S2-N2-C5 & $77.69(11)$ & O3-S2-N2-C4 & $174.25(10)$ \\
O4-S2-N2-C4 & $45.02(11)$ & C25-S2-N2-C4 & $-69.96(11)$ \\
C2-C1-C3-C6 & $49.70(19)$ & C7-C1-C3-C6 & $-137.69(12)$ \\
C2-C1-C3-C4 & $-73.81(18)$ & C7-C1-C3-C4 & $98.79(14)$ \\
C5-N2-C4-C3 & $-50.95(15)$ & S2-N2-C4-C3 & $98.70(12)$ \\
C1-C3-C4-N2 & $179.09(11)$ & C6-C3-C4-N2 & $52.64(15)$ \\
C4-N2-C5-N1 & $53.80(15)$ & S2-N2-C5-N1 & $-95.57(12)$ \\
C6-N1-C5-N2 & $-61.62(15)$ & S1-N1-C5-N2 & $154.29(10)$ \\
C5-N1-C6-C3 & $63.29(14)$ & S1-N1-C6-C3 & $-152.97(9)$ \\
C1-C3-C6-N1 & $179.92(11)$ & $-57.17(14)$ \\
\hline
\end{tabular}




\begin{tabular}{|c|c|c|c|}
\hline C2-C1-C7-C8 & $59.9(2)$ & C3-C1-C7-C8 & $-112.67(15)$ \\
\hline C2-C1-C7-C12 & $-125.44(16)$ & C3-C1-C7-C12 & $61.97(17)$ \\
\hline C12-C7-C8-C9 & $-2.1(2)$ & C1-C7-C8-C9 & $172.64(14)$ \\
\hline C7-C8-C9-C10 & $0.0(2)$ & C8-C9-C10-C11 & $1.3(2)$ \\
\hline C9-C10-C11-C12 & $-0.4(2)$ & $\mathrm{C} 10-\mathrm{C} 11-\mathrm{C} 12-\mathrm{C} 7$ & $-1.8(2)$ \\
\hline $\begin{array}{l}\text { C10-C11-C12- } \\
\text { C13 }\end{array}$ & $177.04(13)$ & C8-C7-C12-C11 & $3.0(2)$ \\
\hline C1-C7-C12-C11 & $-171.70(13)$ & C8-C7-C12-C13 & $-175.82(13)$ \\
\hline C1-C7-C12-C13 & $9.5(2)$ & C11-C12-C13-C14 & $-124.04(16)$ \\
\hline C7-C12-C13-C14 & $54.75(19)$ & C11-C12-C13-C18 & $54.29(19)$ \\
\hline C7-C12-C13-C18 & $-126.92(16)$ & C18-C13-C14-C15 & $0.0(2)$ \\
\hline $\begin{array}{l}\text { C12-C13-C14- } \\
\text { C15 }\end{array}$ & $178.34(15)$ & C13-C14-C15-C16 & $2.0(3)$ \\
\hline $\begin{array}{l}\text { C14-C15-C16- } \\
\text { C17 }\end{array}$ & $-2.2(3)$ & C15-C16-C17-C18 & $0.3(3)$ \\
\hline $\begin{array}{l}\text { C16-C17-C18- } \\
\text { C13 }\end{array}$ & $1.7(3)$ & C14-C13-C18-C17 & $-1.9(2)$ \\
\hline $\begin{array}{l}\text { C12-C13-C18- } \\
\text { C17 }\end{array}$ & $179.76(15)$ & O2-S1-C19-C20 & $-26.52(13)$ \\
\hline O1-S1-C19-C20 & $-158.55(11)$ & N1-S1-C19-C20 & $87.64(12)$ \\
\hline O2-S1-C19-C24 & $154.24(11)$ & O1-S1-C19-C24 & $22.21(13)$ \\
\hline N1-S1-C19-C24 & $-91.60(12)$ & C24-C19-C20-C21 & $0.6(2)$ \\
\hline S1-C19-C20-C21 & $-178.63(11)$ & C19-C20-C21-C22 & $-0.4(2)$ \\
\hline $\begin{array}{l}\text { C20-C21-C22- } \\
\text { C23 }\end{array}$ & $-0.2(2)$ & C20-C21-C22-C31 & $178.10(17)$ \\
\hline $\begin{array}{l}\text { C21-C22-C23- } \\
\text { C24 }\end{array}$ & $0.5(2)$ & $\mathrm{C} 31-\mathrm{C} 22-\mathrm{C} 23-\mathrm{C} 24$ & $-177.70(17)$ \\
\hline
\end{tabular}




$\begin{array}{llll}\text { C22-C23-C24- } & -0.3(2) & \text { C20-C19-C24-C23 } & -0.3(2) \\ \text { C19 } & & \text { O3-S2-C25-C26 } & -144.90(12) \\ \text { S1-C19-C24-C23 } & 178.97(11) & \text { N2-S2-C25-C26 } & 100.37(12) \\ \text { O4-S2-C25-C26 } & -13.85(14) & \text { O4-S2-C25-C30 } & 166.66(11) \\ \text { O3-S2-C25-C30 } & 35.61(13) & \text { C30-C25-C26-C27 } & -0.7(2) \\ \text { N2-S2-C25-C30 } & -79.12(13) & \text { C25-C26-C27-C28 } & 0.9(2) \\ \text { S2-C25-C26-C27 } & 179.86(12) & \text { C26-C27-C28-C32 } & 179.07(15) \\ \text { C26-C27-C28- } & -0.5(2) & & \\ \text { C29 } & & \text { C32-C28-C29-C30 } & -179.68(15) \\ \text { C27-C28-C29- } & -0.1(2) & & \\ \text { C30 } & & \text { C26-C25-C30-C29 } & 0.1(2) \\ \text { C28-C29-C30- } & 0.3(2) & & \\ \text { C25 } & & & \\ \text { S2-C25-C30-C29 } & 179.54(11) & & \end{array}$

Table S15. Anisotropic atomic displacement parameters $\left(\AA^{2}\right)$ for $i 2791 \_1$.

The anisotropic atomic displacement factor exponent takes the form: $2 \pi^{2}\left[h^{2} a^{* 2} U_{11}+\ldots+2 h k a^{*} b^{*} U_{12}\right]$

$\begin{array}{llllll}\mathbf{U}_{11} & \mathrm{U}_{22} & \mathrm{U}_{33} & \mathrm{U}_{23} & \mathrm{U}_{13} & \mathrm{U}_{12}\end{array}$

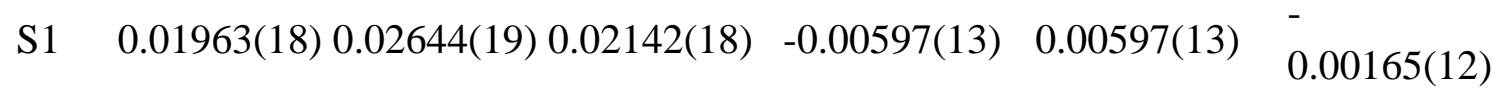

S2 $\quad 0.02059(18) 0.02946(19) 0.01665(17) \quad 0.00008(12) \quad-0.00004(12) \quad 0.00141(13)$

$\begin{array}{lllllll}\mathrm{N} 1 & 0.0156(5) & 0.0289(6) & 0.0186(6) & -0.0036(5) & 0.0039(4) & -0.0001(5)\end{array}$

$\begin{array}{lllllll}\mathrm{N} 2 & 0.0167(6) & 0.0264(6) & 0.0199(6) & -0.0033(5) & 0.0020(4) & -0.0004(4)\end{array}$

$\begin{array}{lllllll}\mathrm{O} 1 & 0.0267(5) & 0.0244(5) & 0.0369(6) & -0.0035(4) & 0.0125(4) & 0.0013(4)\end{array}$

$\begin{array}{lllllll}\mathrm{O} 2 & 0.0270(6) & 0.0477(7) & 0.0238(5) & -0.0130(5) & 0.0040(4) & -0.0063(5)\end{array}$ 


$\begin{array}{lccccccc} & \mathbf{U}_{11} & \mathbf{U}_{22} & \mathbf{U}_{33} & \mathbf{U}_{23} & \mathbf{U}_{13} & \mathbf{U}_{12} \\ \text { O3 } & 0.0232(5) & 0.0402(6) & 0.0283(5) & 0.0031(5) & -0.0022(4) & 0.0060(5) \\ \text { O4 } & 0.0298(6) & 0.0384(6) & 0.0166(5) & -0.0011(4) & 0.0019(4) & -0.0030(4) \\ \text { C1 } & 0.0175(6) & 0.0256(7) & 0.0225(7) & -0.0049(5) & 0.0010(5) & 0.0012(5) \\ \text { C2 } & 0.0221(7) & 0.0250(7) & 0.0428(9) & -0.0089(6) & 0.0016(6) & 0.0007(6) \\ \text { C3 } & 0.0152(6) & 0.0214(7) & 0.0209(6) & -0.0049(5) & 0.0014(5) & -0.0006(5) \\ \text { C4 } & 0.0170(6) & 0.0259(7) & 0.0197(6) & -0.0053(5) & 0.0026(5) & -0.0003(5) \\ \text { C5 } & 0.0154(6) & 0.0289(7) & 0.0216(7) & -0.0033(5) & 0.0017(5) & -0.0034(5) \\ \text { C6 } & 0.0184(6) & 0.0265(7) & 0.0215(7) & -0.0018(5) & 0.0021(5) & 0.0009(5) \\ \text { C7 } & 0.0161(6) & 0.0224(7) & 0.0275(7) & -0.0005(5) & 0.0015(5) & 0.0038(5) \\ \text { C8 } & 0.0235(7) & 0.0357(8) & 0.0300(8) & -0.0007(6) & 0.0044(6) & 0.0067(6) \\ \text { C9 } & 0.0260(8) & 0.0451(10) & 0.0394(9) & 0.0093(8) & 0.0127(7) & 0.0077(7) \\ \text { C10 } & 0.0224(7) & 0.0297(8) & 0.0542(11) & 0.0133(7) & 0.0076(7) & 0.0023(6) \\ \text { C11 } & 0.0205(7) & 0.0226(7) & 0.0439(9) & 0.0026(6) & -0.0009(6) & 0.0003(6) \\ \text { C12 } & 0.0156(6) & 0.0190(6) & 0.0325(8) & 0.0004(6) & -0.0020(5) & 0.0028(5) \\ \text { C13 } & 0.0188(6) & 0.0235(7) & 0.0262(7) & -0.0052(6) & -0.0045(5) & -0.0015(5) \\ \text { C14 } & 0.0384(9) & 0.0248(8) & 0.0301(8) & -0.0043(6) & 0.0010(7) & 0.0000(6) \\ \text { C15 } & 0.0570(11) & 0.0320(9) & 0.0267(8) & -0.0019(7) & 0.0019(7) & -0.0070(8) \\ \text { C16 } & 0.0465(10) & 0.0464(10) & 0.0261(8) & -0.0109(7) & 0.0048(7) & -0.0035(8) \\ \text { C17 } & 0.0475(10) & 0.0393(9) & 0.0326(9) & -0.0119(7) & -0.0009(7) & 0.0130(8) \\ \text { C18 } & 0.0388(9) & 0.0293(8) & 0.0303(8) & -0.0036(6) & -0.0031(7) & 0.0087(7) \\ \text { C19 } & 0.0212(7) & 0.0253(7) & 0.0182(6) & -0.0022(5) & 0.0063(5) & 0.0017(5) \\ \text { C20 } & 0.0291(8) & 0.0361(8) & 0.0202(7) & 0.0014(6) & 0.0019(6) & 0.0059(6) \\ \text { C21 } & 0.0432(9) & 0.0319(8) & 0.0264(8) & 0.0064(6) & 0.0080(7) & 0.0085(7)\end{array}$




$\begin{array}{ccccccc} & \mathbf{U}_{11} & \mathbf{U}_{22} & \mathbf{U}_{33} & \mathbf{U}_{23} & \mathbf{U}_{13} & \mathbf{U}_{12} \\ \text { C22 } & 0.0404(9) & 0.0252(8) & 0.0371(9) & -0.0004(6) & 0.0189(7) & -0.0004(7) \\ \text { C23 } & 0.0235(7) & 0.0309(8) & 0.0394(9) & -0.0037(7) & 0.0073(6) & -0.0015(6) \\ \text { C24 } & 0.0200(7) & 0.0263(7) & 0.0278(7) & -0.0003(6) & 0.0040(6) & 0.0027(5) \\ \text { C25 } & 0.0259(7) & 0.0212(7) & 0.0188(6) & 0.0015(5) & 0.0016(5) & 0.0024(5) \\ \text { C26 } & 0.0299(7) & 0.0262(7) & 0.0203(7) & -0.0002(5) & 0.0071(6) & -0.0003(6) \\ \text { C27 } & 0.0301(8) & 0.0280(8) & 0.0250(7) & 0.0009(6) & 0.0062(6) & -0.0041(6) \\ \text { C28 } & 0.0363(8) & 0.0210(7) & 0.0211(7) & 0.0014(5) & 0.0032(6) & 0.0005(6) \\ \text { C29 } & 0.0359(8) & 0.0252(7) & 0.0205(7) & -0.0001(5) & 0.0081(6) & 0.0042(6) \\ \text { C30 } & 0.0267(7) & 0.0253(7) & 0.0245(7) & 0.0017(6) & 0.0067(6) & 0.0034(6) \\ \text { C31 } & 0.0612(13) & 0.0310(10) & 0.0842(16) & 0.0063(10) & 0.0275(12) & -0.0073(9) \\ \text { C32 } & 0.0471(10) & 0.0336(9) & 0.0296(8) & -0.0069(7) & 0.0081(7) & -0.0088(7)\end{array}$

Table S16. Hydrogen atomic coordinates and isotropic atomic displacement parameters $\left(\AA^{2}\right)$ for i2791_1.

\begin{tabular}{lrrrr}
\hline & & & & \\
& $\mathbf{x} / \mathbf{a}$ & $\mathbf{y} / \mathbf{b}$ & $\mathbf{z} / \mathbf{c}$ & $\mathbf{U}(\mathbf{e q})$ \\
$\mathrm{H} 2 \mathrm{~A}$ & 0.8994 & 0.1027 & 0.2985 & 0.036 \\
$\mathrm{H} 2 \mathrm{~B}$ & 0.7522 & 0.0962 & 0.3093 & 0.036 \\
$\mathrm{H} 3$ & 0.7148 & 0.3379 & 0.3153 & 0.023 \\
$\mathrm{H} 4 \mathrm{~A}$ & 0.6821 & 0.2944 & 0.1908 & 0.025 \\
$\mathrm{H} 4 \mathrm{~B}$ & 0.6172 & 0.2023 & 0.2130 & 0.025 \\
$\mathrm{H} 5 \mathrm{~A}$ & 0.4084 & 0.2220 & 0.2718 & 0.026 \\
$\mathrm{H} 5 \mathrm{~B}$ & 0.3582 & 0.3238 & 0.2762 & 0.026
\end{tabular}




\begin{tabular}{|c|c|c|c|c|}
\hline & $\mathbf{x} / \mathbf{a}$ & $\mathbf{y} / \mathbf{b}$ & $\mathbf{z} / \mathbf{c}$ & $\mathbf{U}(\mathbf{e q})$ \\
\hline H6A & 0.5890 & 0.1754 & 0.3531 & 0.027 \\
\hline H6B & 0.6506 & 0.2464 & 0.4123 & 0.027 \\
\hline H8 & 0.9665 & 0.2303 & 0.1990 & 0.036 \\
\hline H9 & 1.1216 & 0.3340 & 0.1783 & 0.044 \\
\hline H10 & 1.1714 & 0.4481 & 0.2644 & 0.042 \\
\hline H11 & 1.0725 & 0.4565 & 0.3722 & 0.035 \\
\hline H14 & 0.9348 & 0.2239 & 0.4569 & 0.037 \\
\hline H15 & 0.8520 & 0.2370 & 0.5702 & 0.046 \\
\hline H16 & 0.7589 & 0.3717 & 0.6029 & 0.048 \\
\hline H17 & 0.7608 & 0.4969 & 0.5243 & 0.048 \\
\hline H18 & 0.8531 & 0.4868 & 0.4139 & 0.04 \\
\hline $\mathrm{H} 20$ & 0.4774 & 0.1542 & 0.5042 & 0.034 \\
\hline $\mathrm{H} 21$ & 0.3751 & 0.0183 & 0.5204 & 0.04 \\
\hline $\mathrm{H} 23$ & 0.0828 & 0.1094 & 0.3913 & 0.037 \\
\hline $\mathrm{H} 24$ & 0.1842 & 0.2461 & 0.3730 & 0.03 \\
\hline $\mathrm{H} 26$ & 0.7330 & 0.5008 & 0.1956 & 0.03 \\
\hline $\mathrm{H} 27$ & 0.8350 & 0.6029 & 0.2775 & 0.033 \\
\hline $\mathrm{H} 29$ & 0.5466 & 0.6028 & 0.4028 & 0.032 \\
\hline H30 & 0.4421 & 0.5031 & 0.3206 & 0.03 \\
\hline H31A & 0.2094 & -0.0888 & 0.4521 & 0.086 \\
\hline H31B & 0.0787 & -0.0410 & 0.4367 & 0.086 \\
\hline $\mathrm{H} 31 \mathrm{C}$ & 0.1418 & -0.0476 & 0.5193 & 0.086 \\
\hline H32A & 0.8532 & 0.6835 & 0.3908 & 0.055 \\
\hline
\end{tabular}




$\begin{array}{ccccc} & \mathbf{x} / \mathbf{a} & \mathbf{y} / \mathbf{b} & \mathbf{z} / \mathbf{c} & \mathbf{U}(\mathbf{e q}) \\ \text { H32B } & 0.7661 & 0.6537 & 0.4529 & 0.055 \\ \text { H32C } & 0.7280 & 0.7368 & 0.3992 & 0.055\end{array}$


25. NMR Spectra 
${ }^{1} \mathrm{H}$ NMR (500 MHz, $\mathrm{CDCl}_{3}$ ) of compound 3a

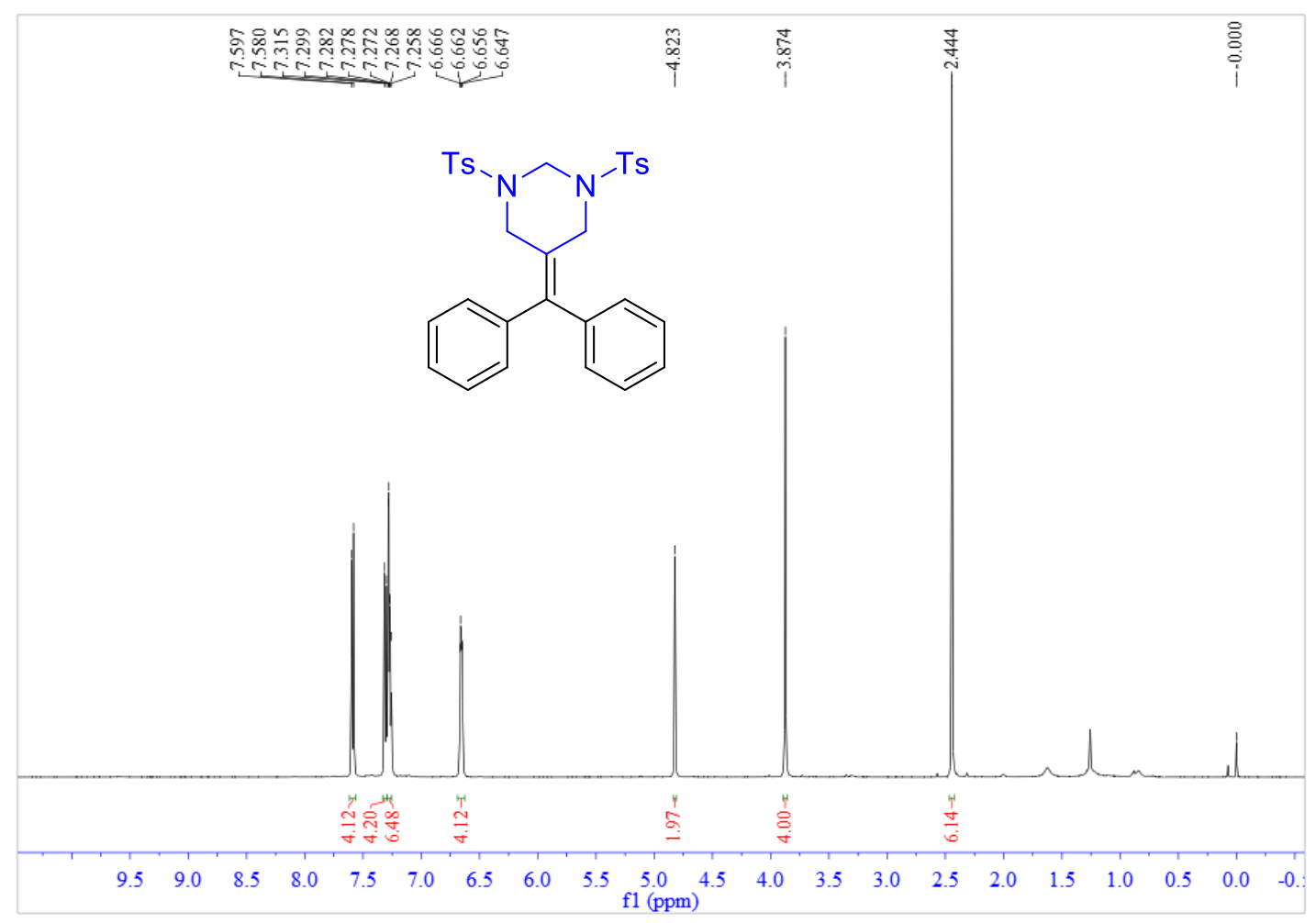

${ }^{13} \mathrm{C}$ NMR (125 MHz, $\mathrm{CDCl}_{3}$ ) of compound 3a

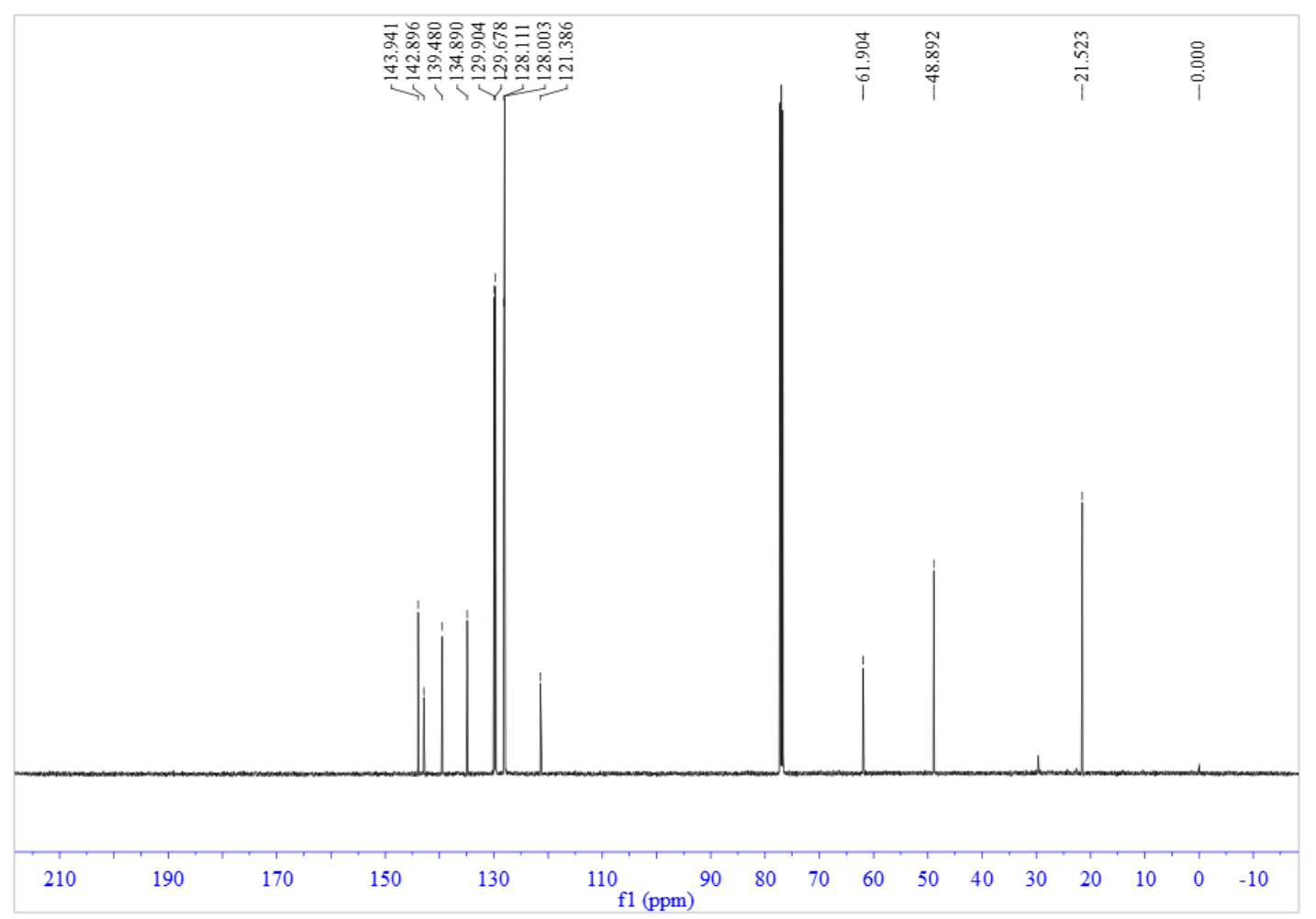


${ }^{13} \mathrm{C}$ APT of compound $\mathbf{3 a}$

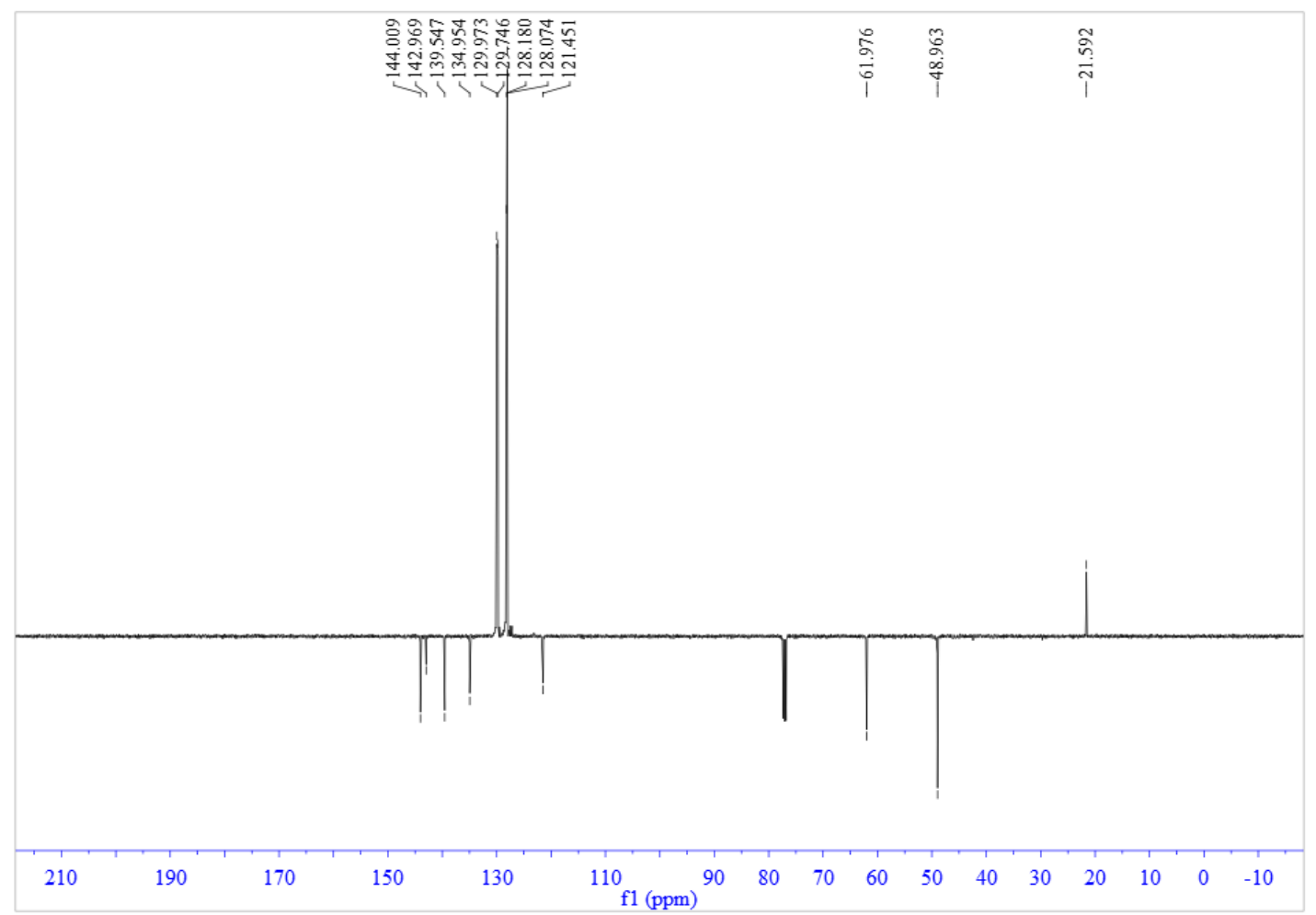


${ }^{1} \mathrm{H}$ NMR $\left(500 \mathrm{MHz}, \mathrm{CDCl}_{3}\right.$ ) of compound $\mathbf{3 b}$

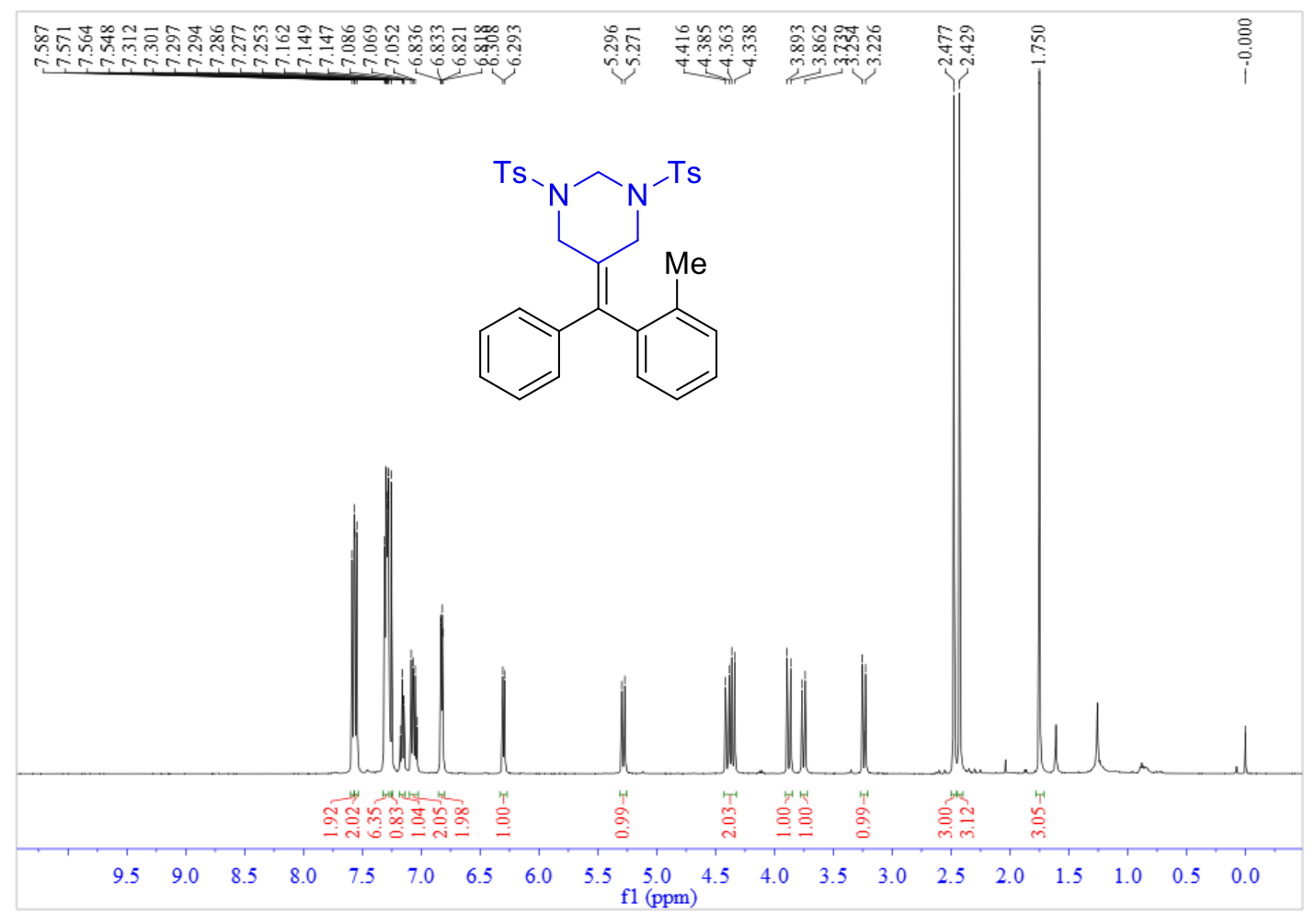

${ }^{13} \mathrm{C}$ NMR (125 MHz, $\mathrm{CDCl}_{3}$ ) of compound $\mathbf{3 b}$

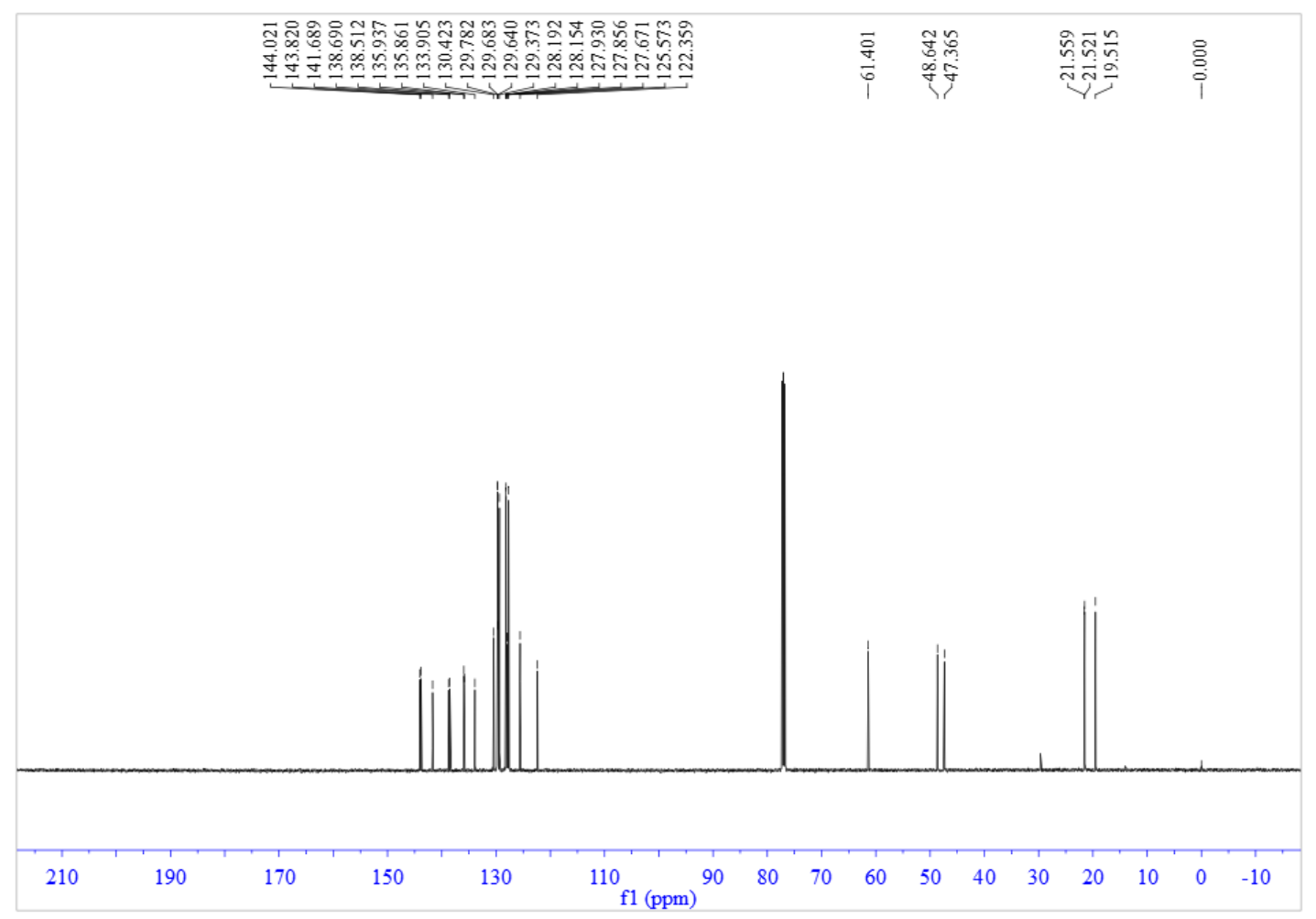


${ }^{1} \mathrm{H}$ NMR $\left(500 \mathrm{MHz}, \mathrm{CDCl}_{3}\right.$ ) of compound $\mathbf{3 c}$

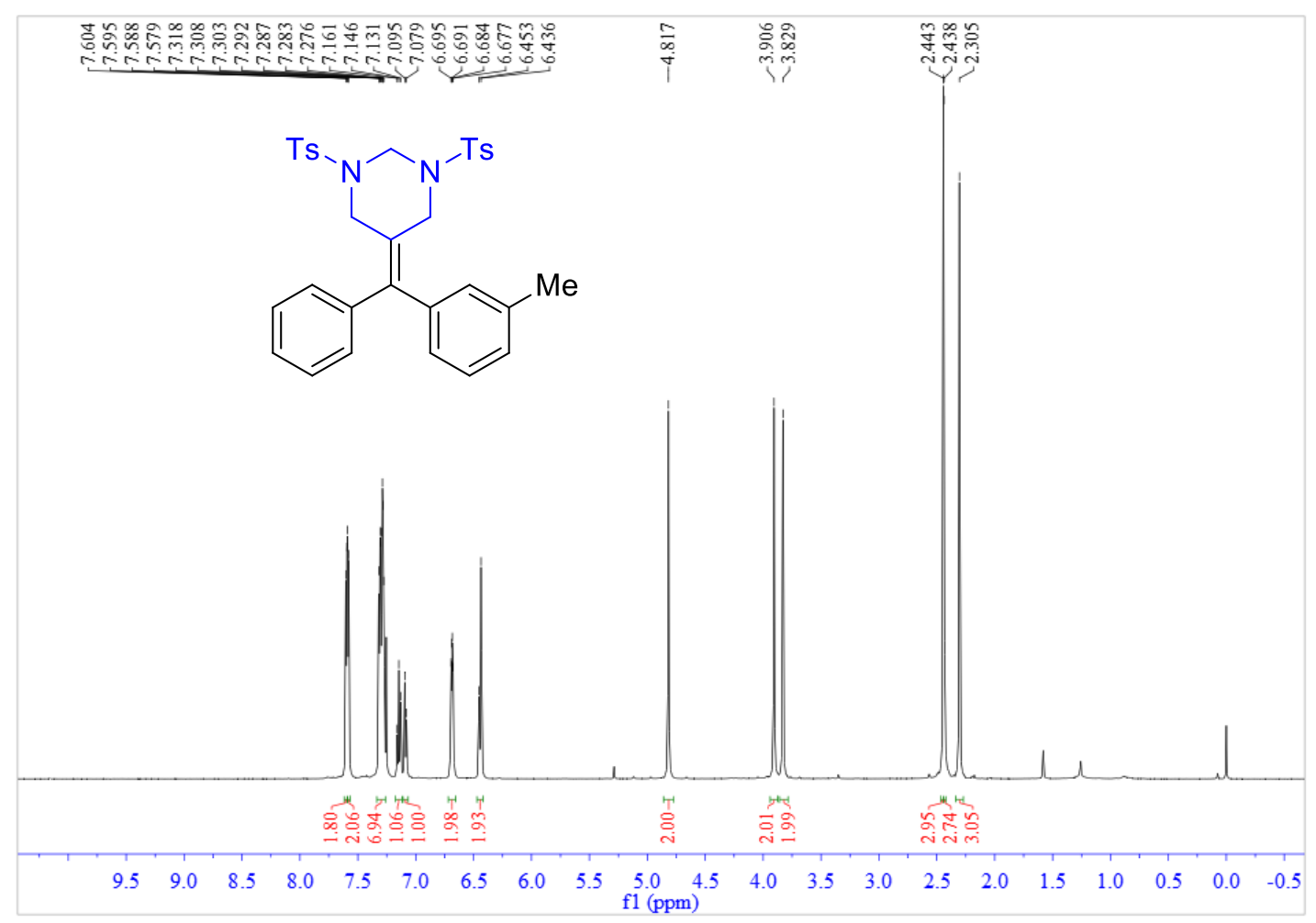

${ }^{13} \mathrm{C}$ NMR (125 MHz, $\mathrm{CDCl}_{3}$ ) of compound 3c

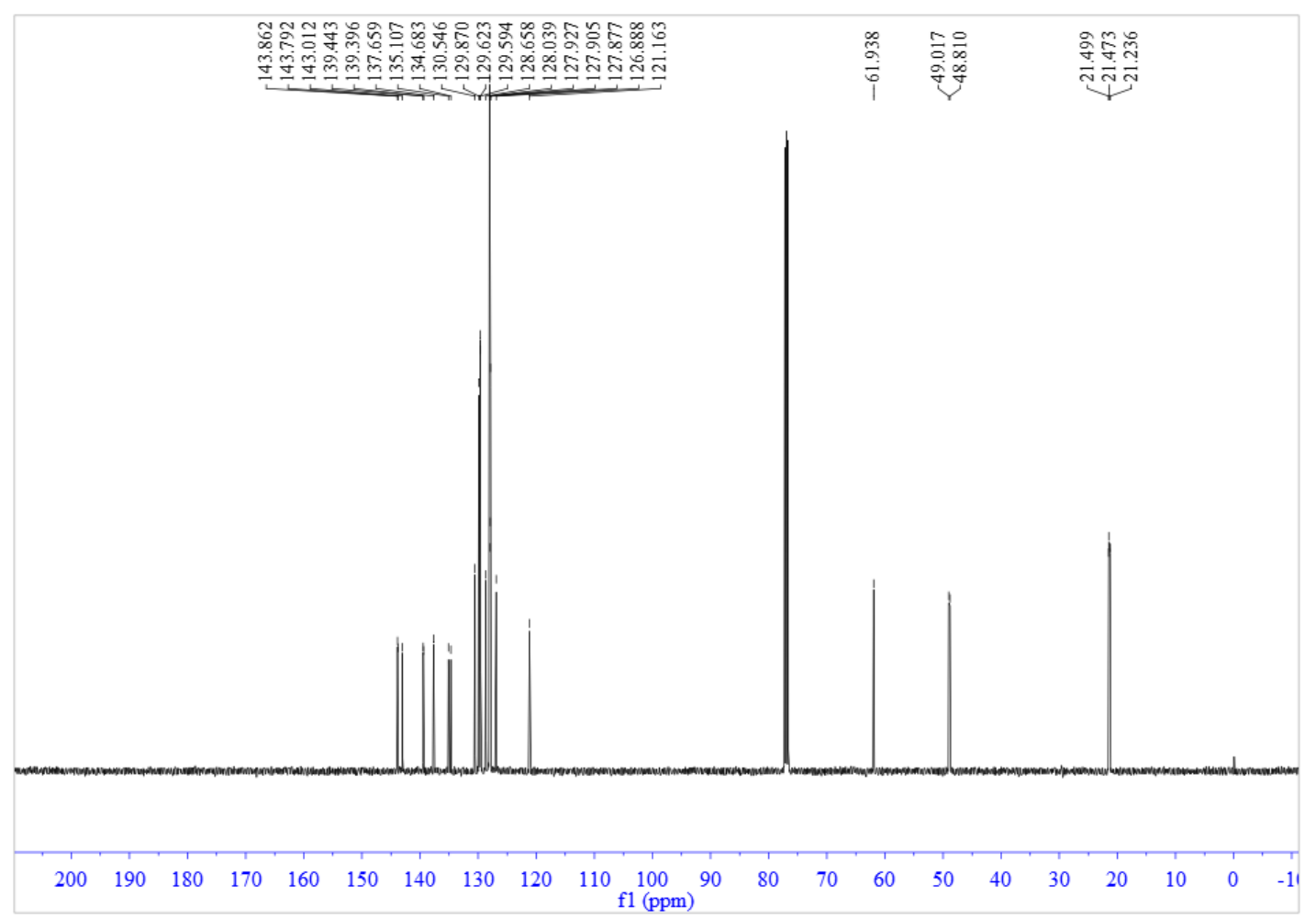


${ }^{1} \mathrm{H}$ NMR $\left(500 \mathrm{MHz}, \mathrm{CDCl}_{3}\right)$ of compound $\mathbf{3 d}$

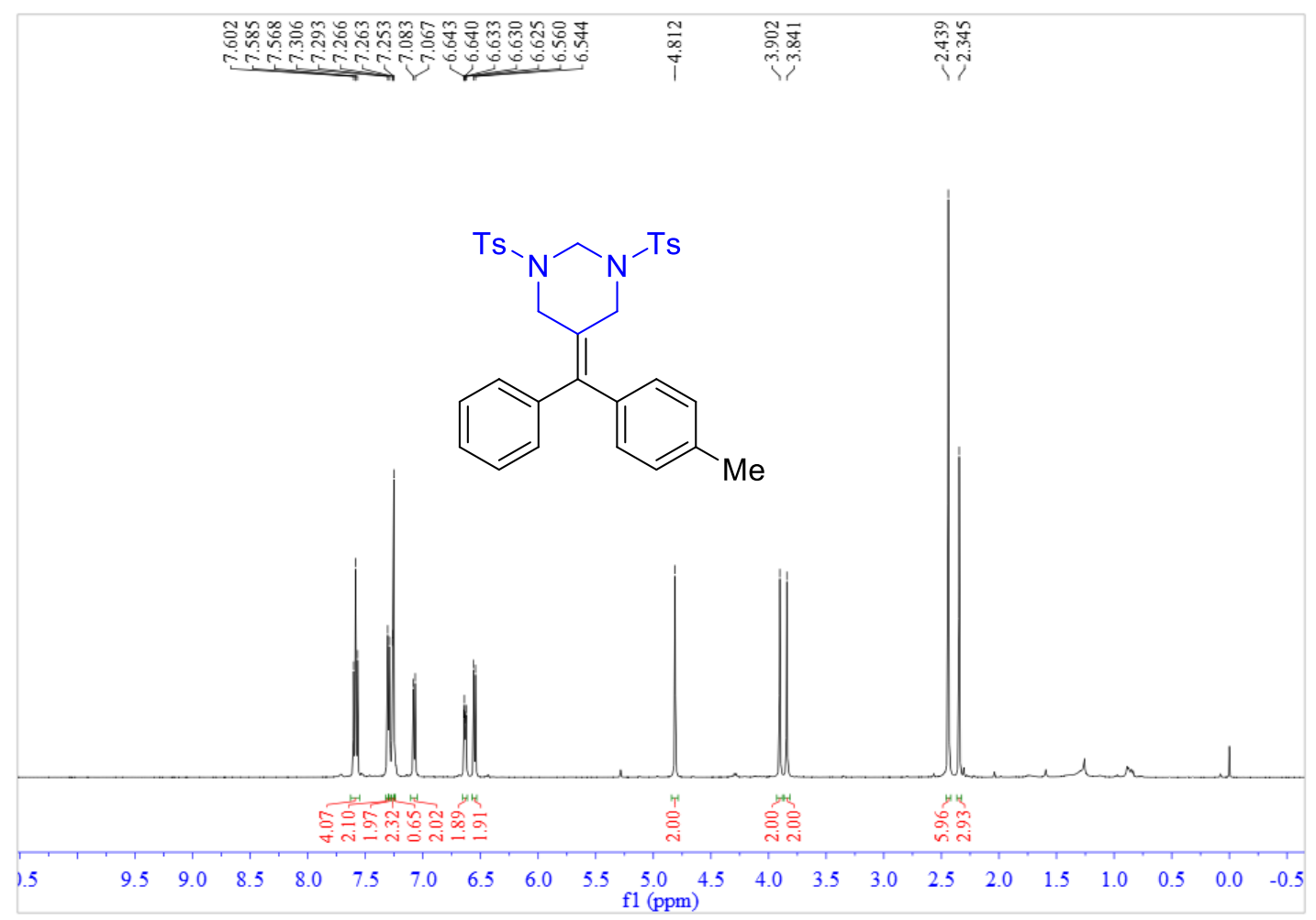

${ }^{13} \mathrm{C}$ NMR (125 MHz, $\mathrm{CDCl}_{3}$ ) of compound $\mathbf{3 d}$

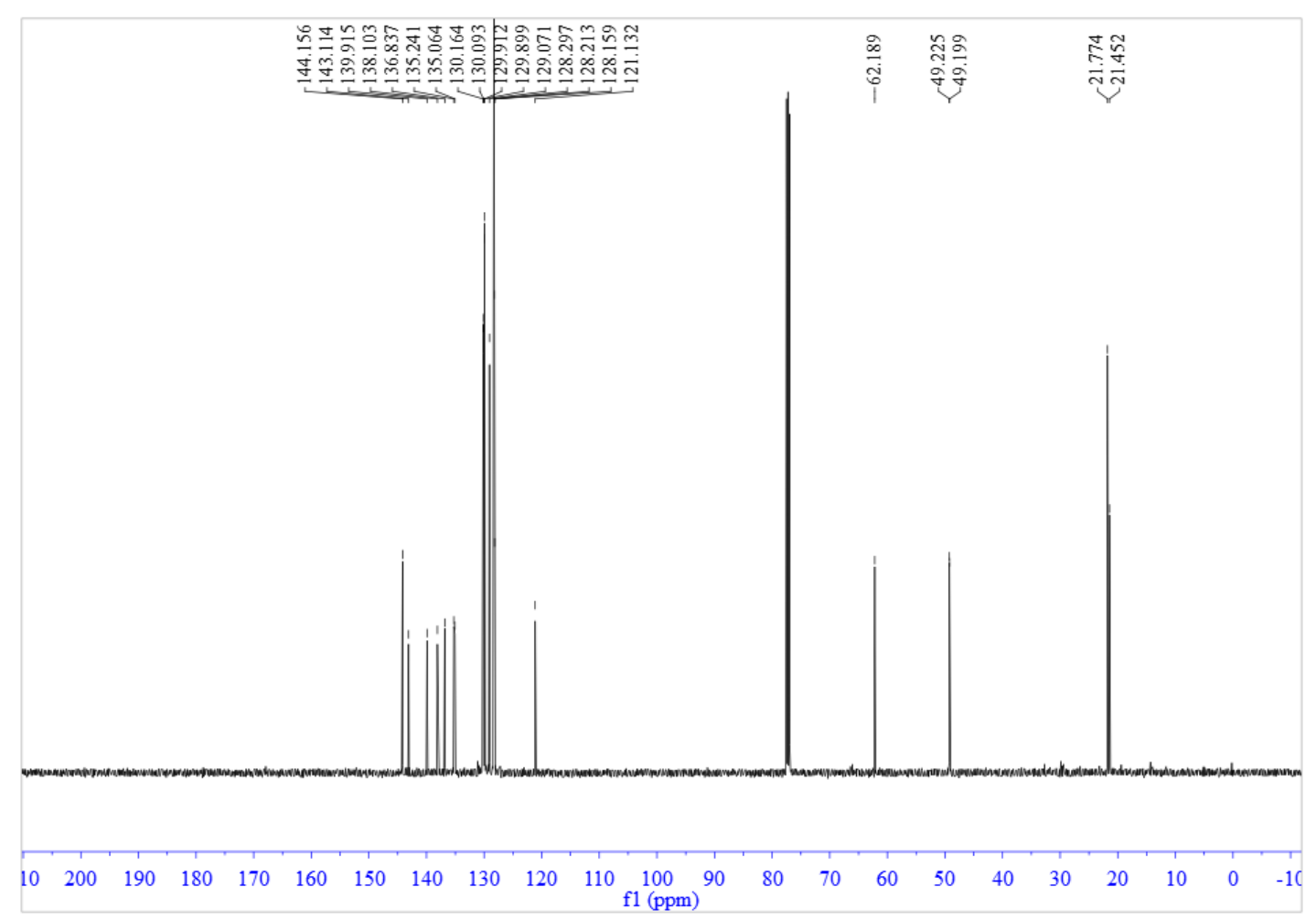


${ }^{1} \mathrm{H}$ NMR $\left(500 \mathrm{MHz}, \mathrm{CDCl}_{3}\right.$ ) of compound $\mathbf{3 e}$

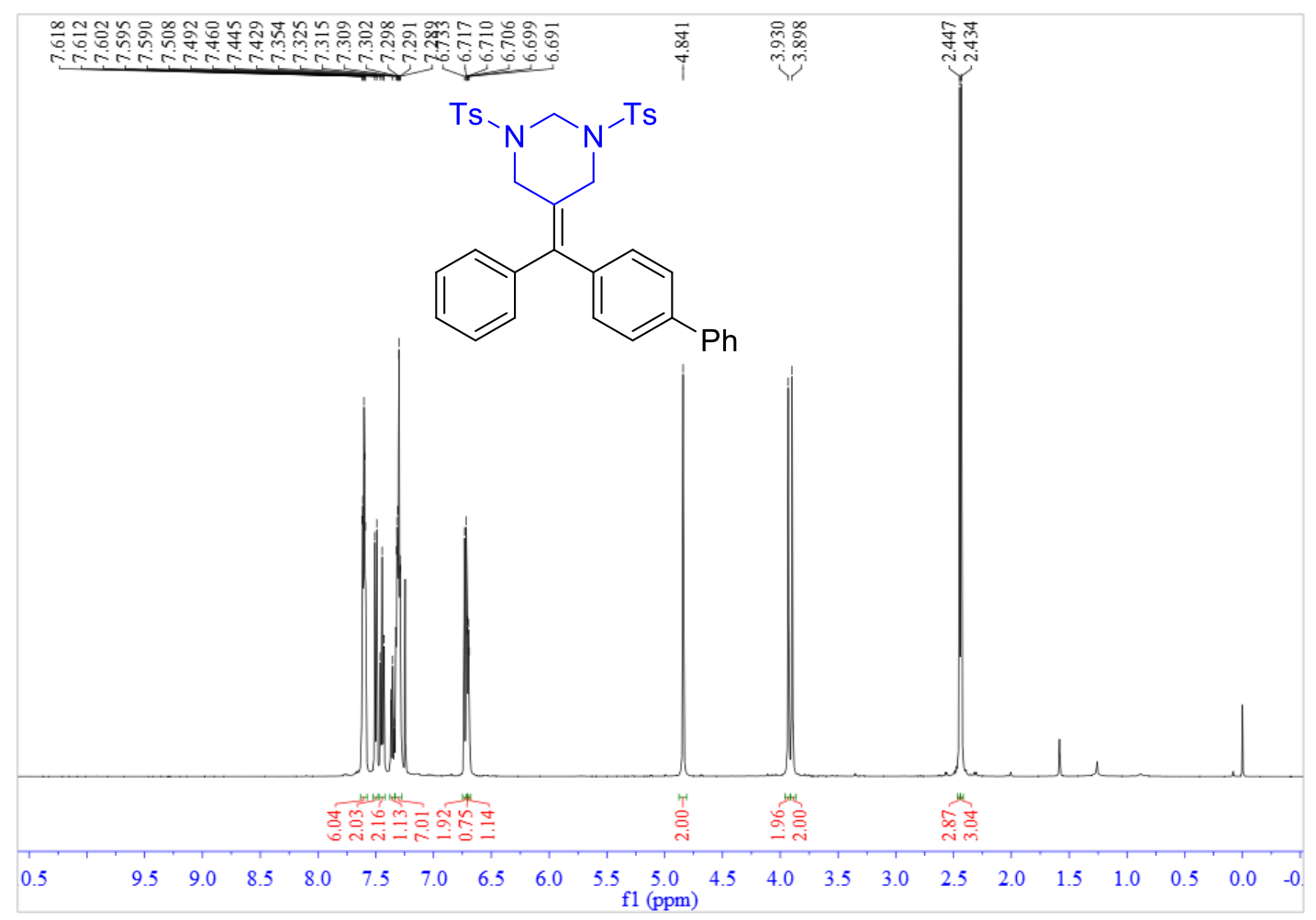

${ }^{13} \mathrm{C}$ NMR $\left(150 \mathrm{MHz}, \mathrm{CDCl}_{3}\right)$ of compound $\mathbf{3 e}$

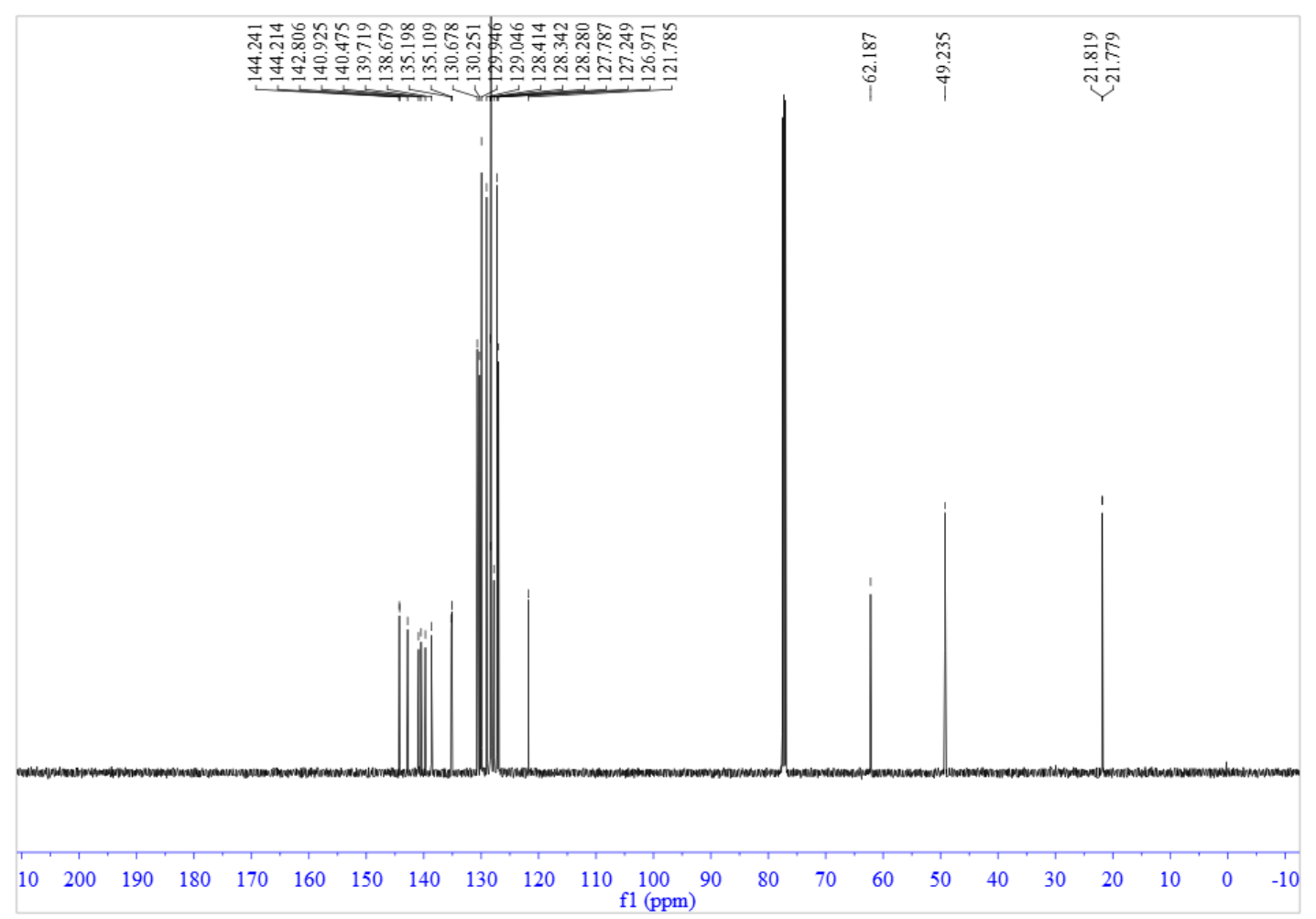


${ }^{1} \mathrm{H}$ NMR $\left(500 \mathrm{MHz}, \mathrm{CDCl}_{3}\right.$ ) of compound $\mathbf{3 f}$

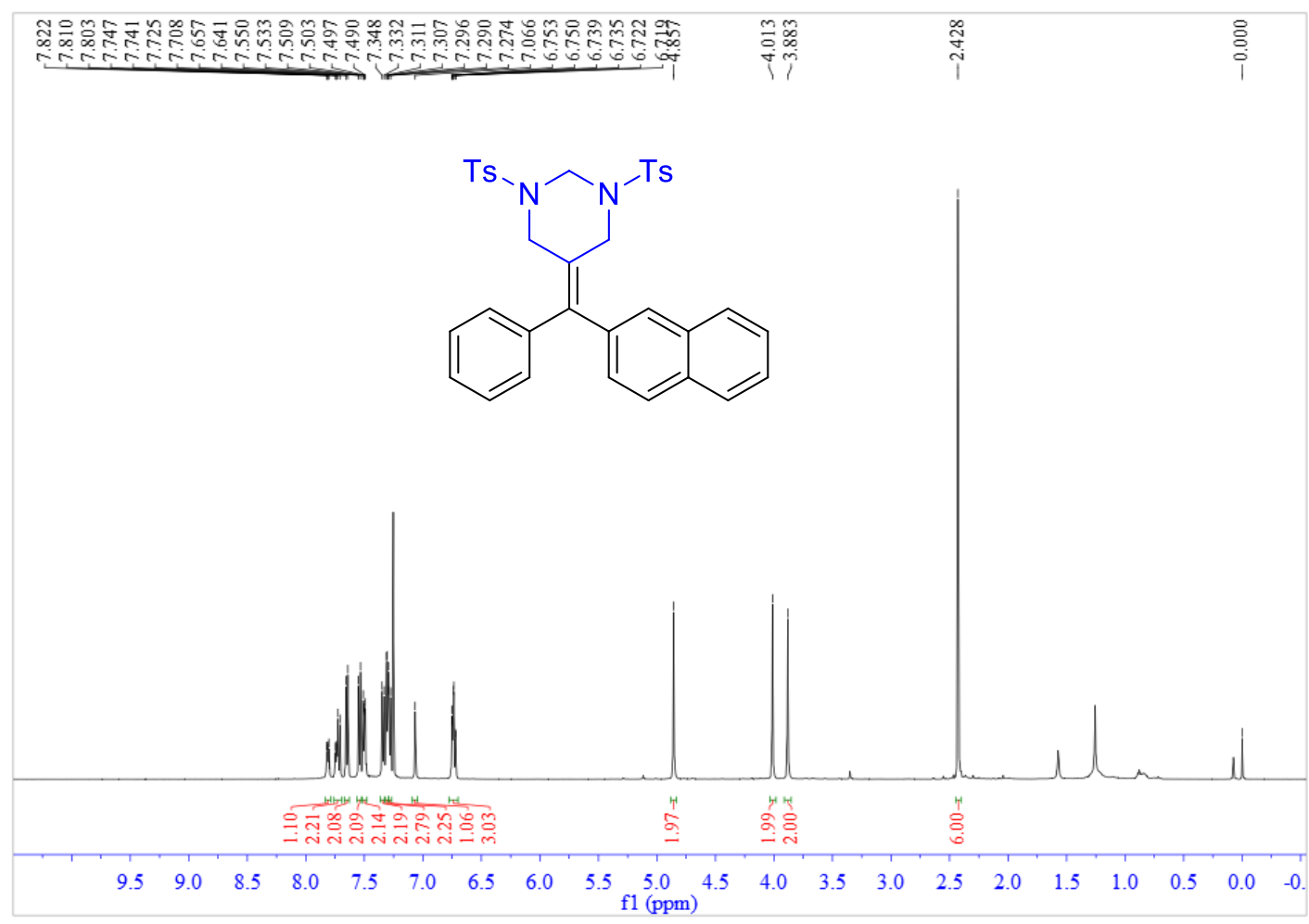

${ }^{13} \mathrm{C}$ NMR $\left(150 \mathrm{MHz}, \mathrm{CDCl}_{3}\right)$ of compound $\mathbf{3 f}$

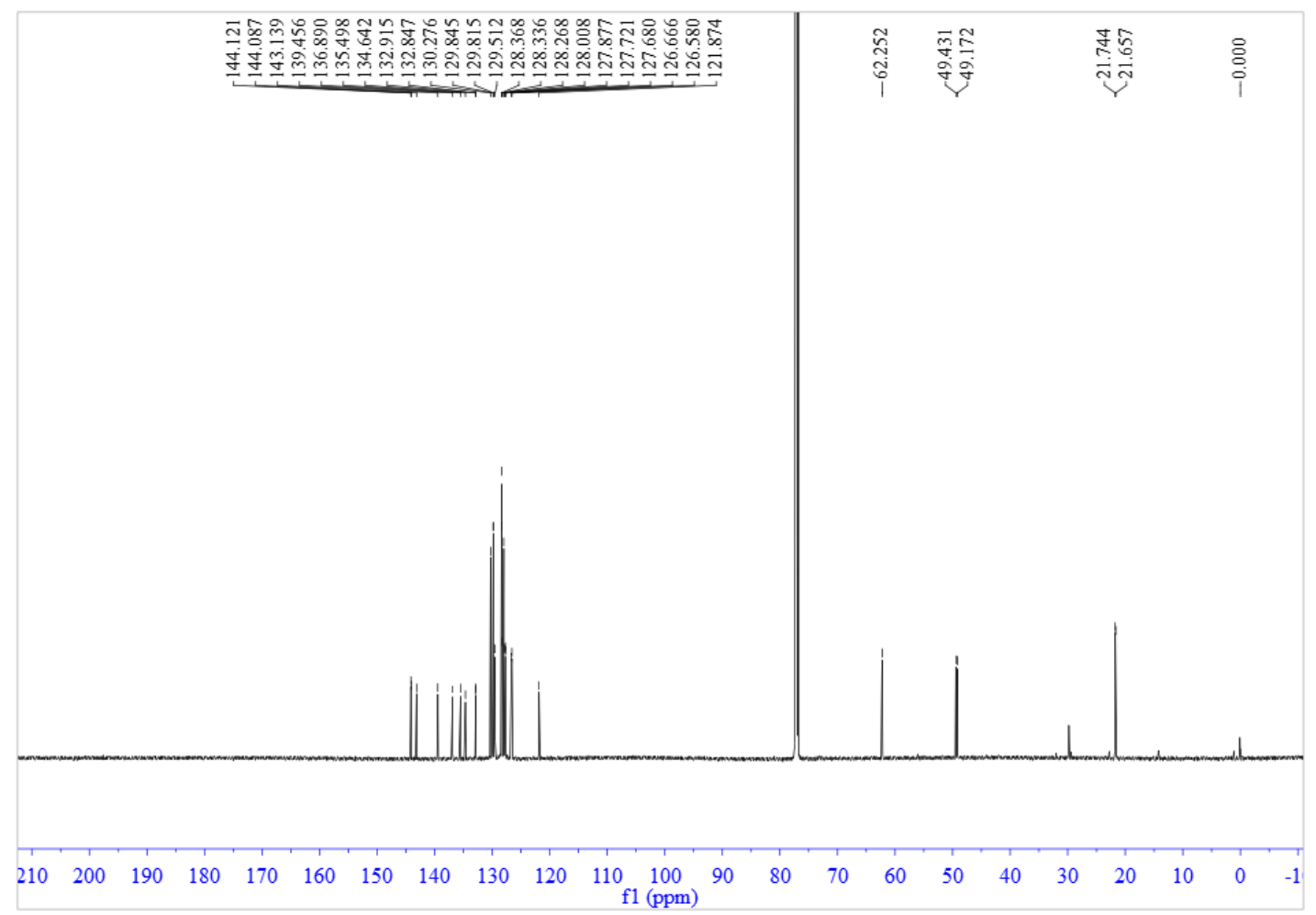


${ }^{1} \mathrm{H}$ NMR $\left(500 \mathrm{MHz}, \mathrm{CDCl}_{3}\right.$ ) of compound $\mathbf{3 g}$

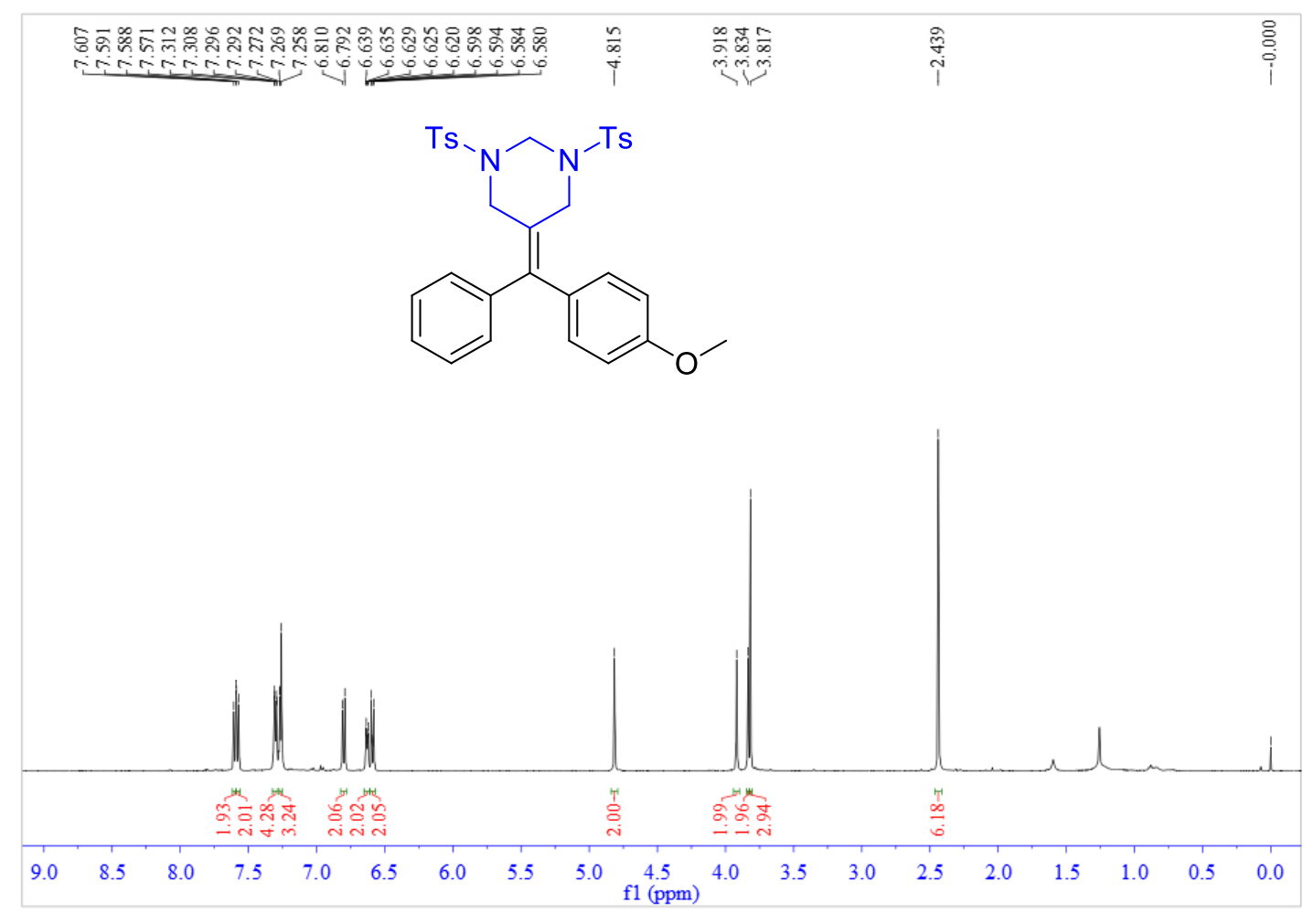

${ }^{13} \mathrm{C}$ NMR $\left(125 \mathrm{MHz}, \mathrm{CDCl}_{3}\right.$ ) of compound $\mathbf{3 g}$

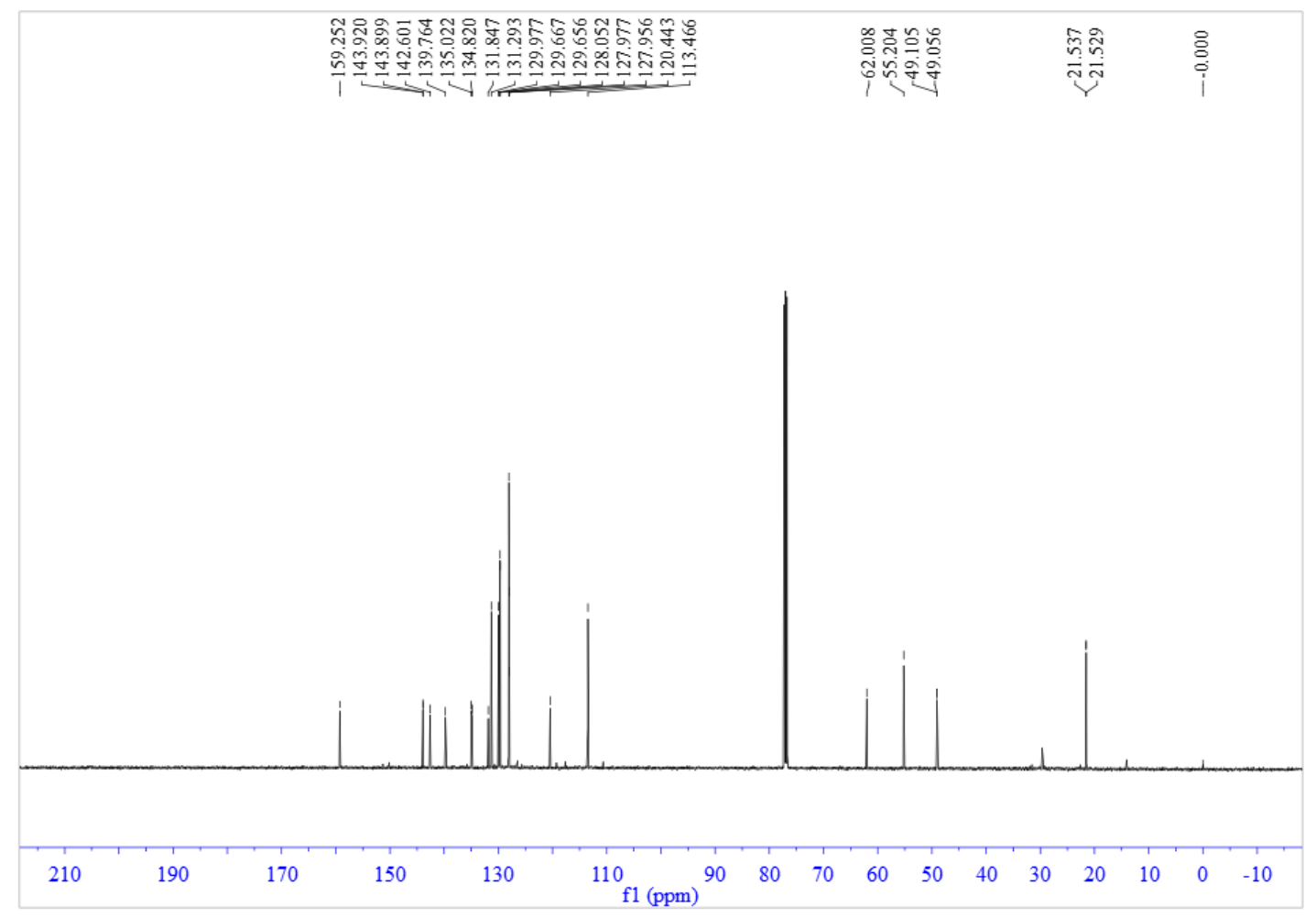




\section{${ }^{1} \mathrm{H}$ NMR $\left(500 \mathrm{MHz}, \mathrm{CDCl}_{3}\right)$ of compound $\mathbf{3 h}$}

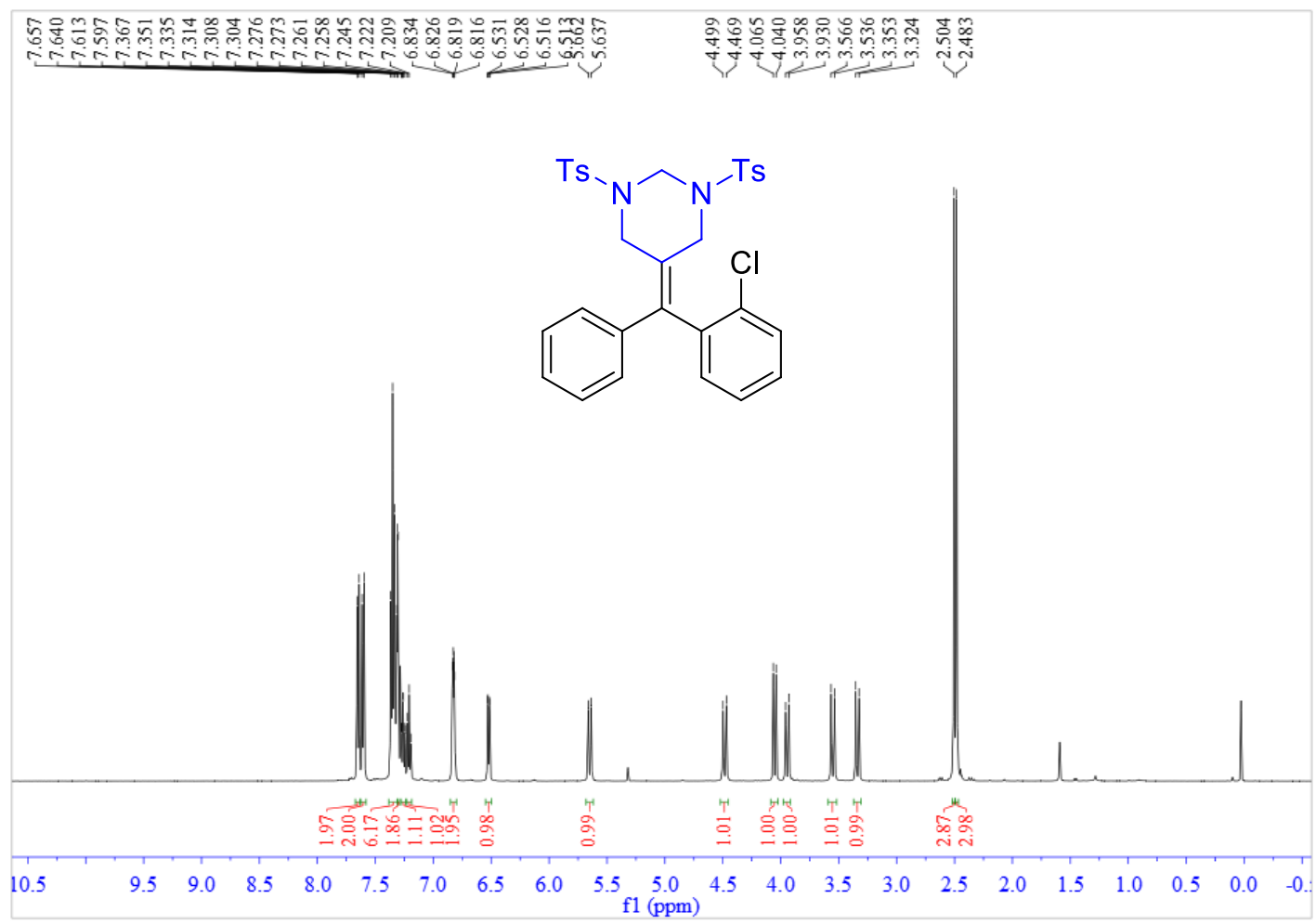

${ }^{13} \mathrm{C}$ NMR (125 MHz, $\left.\mathrm{CDCl}_{3}\right)$ of compound $\mathbf{3 h}$

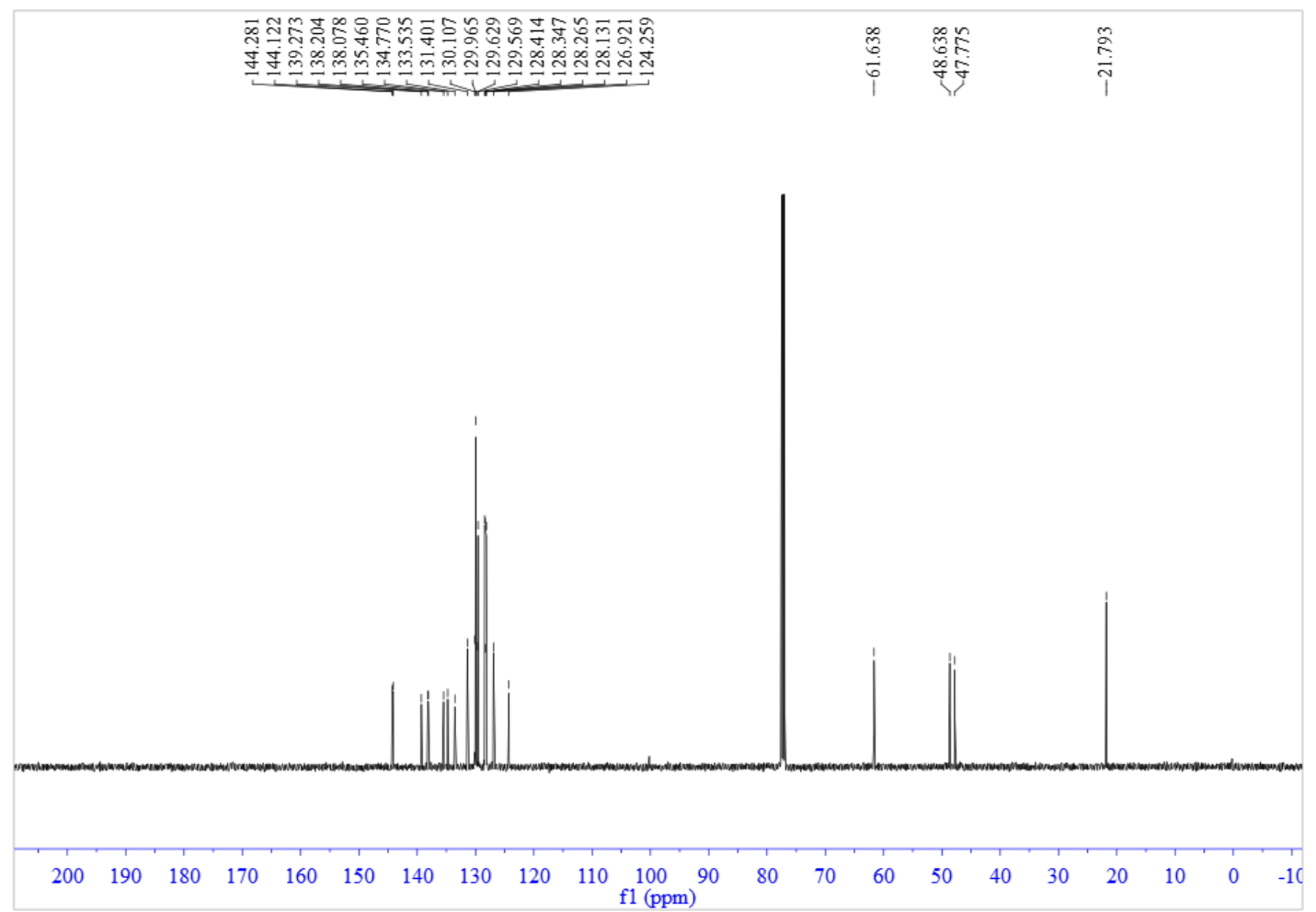


${ }^{1} \mathrm{H}$ NMR (500 MHz, $\mathrm{CDCl}_{3}$ ) of compound $\mathbf{3 i}$

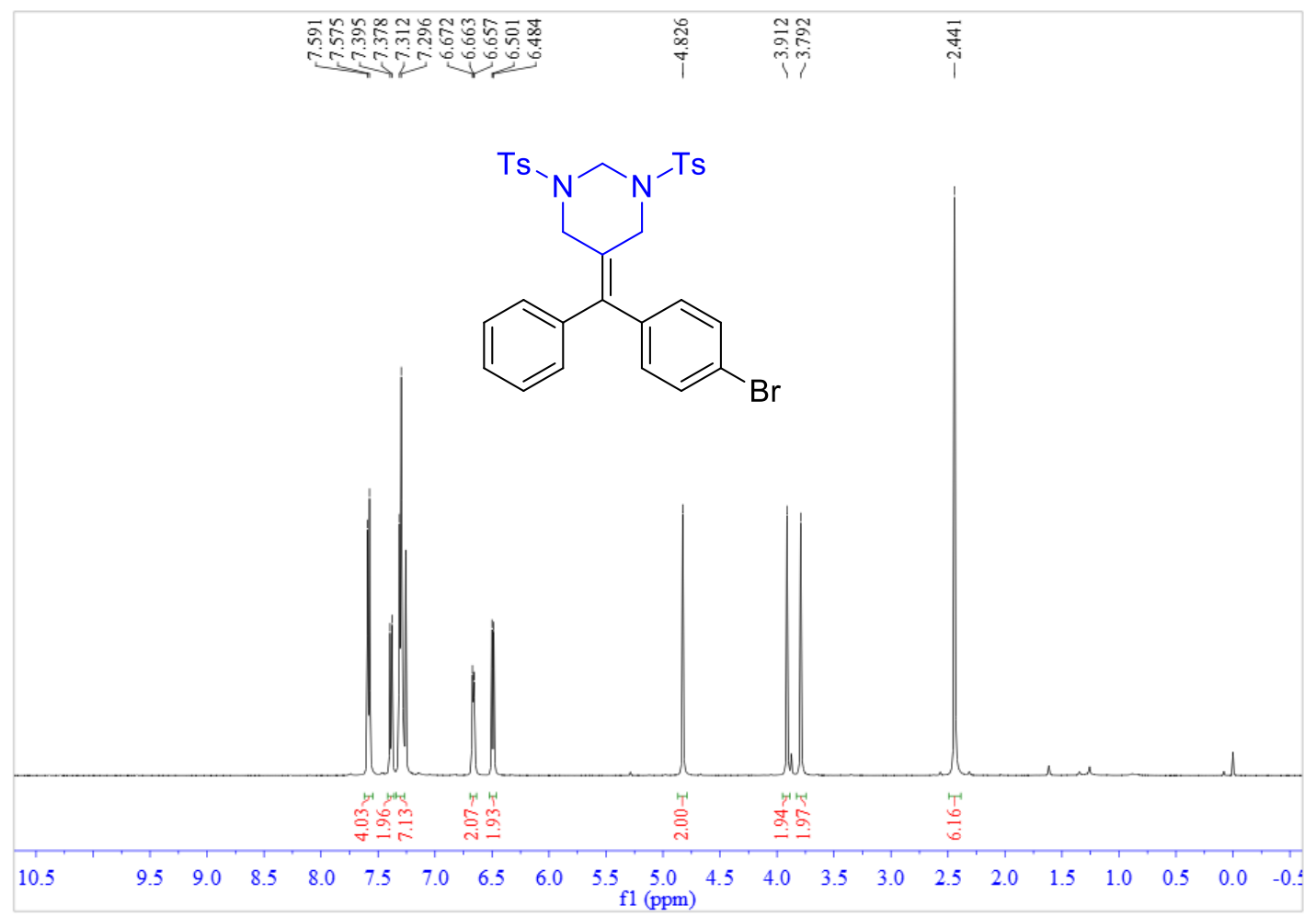

${ }^{13} \mathrm{C}$ NMR (125 MHz, $\left.\mathrm{CDCl}_{3}\right)$ of compound 3i

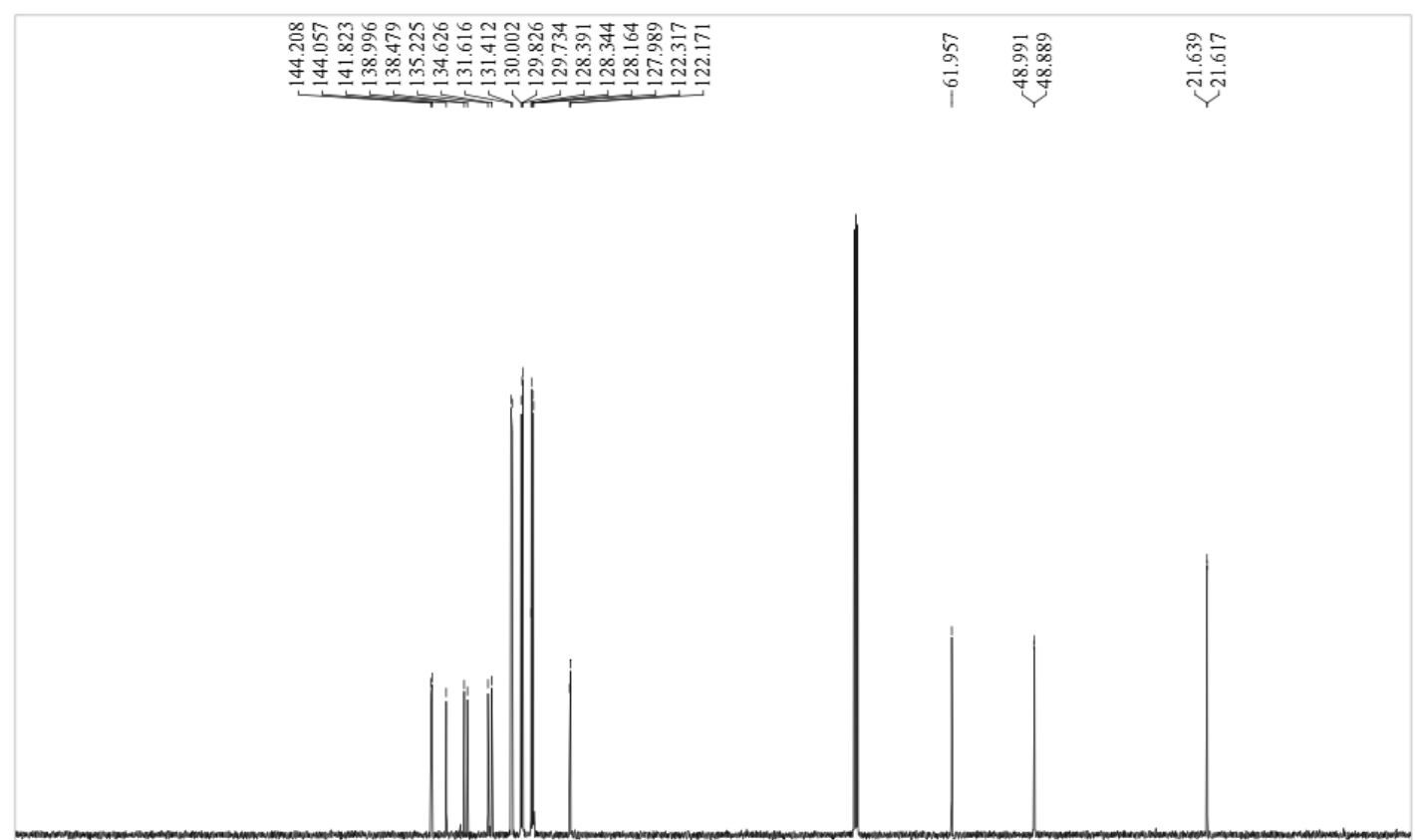

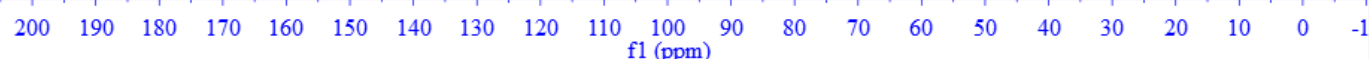


${ }^{1} \mathrm{H}$ NMR (500 MHz, $\mathrm{CDCl}_{3}$ ) of compound $\mathbf{3 j}$

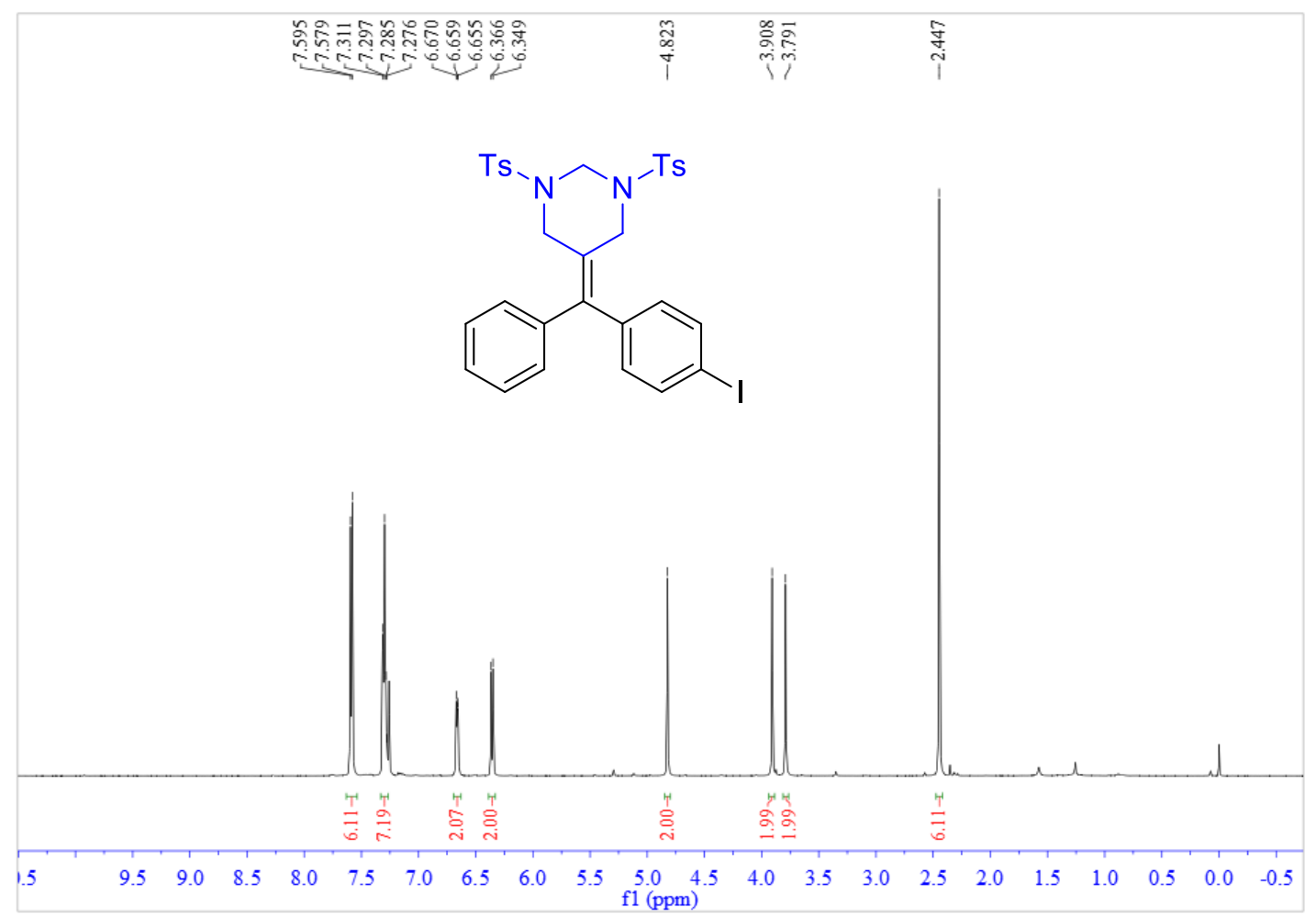

${ }^{13} \mathrm{C}$ NMR (125 MHz, $\mathrm{CDCl}_{3}$ ) of compound $\mathbf{3 j}$

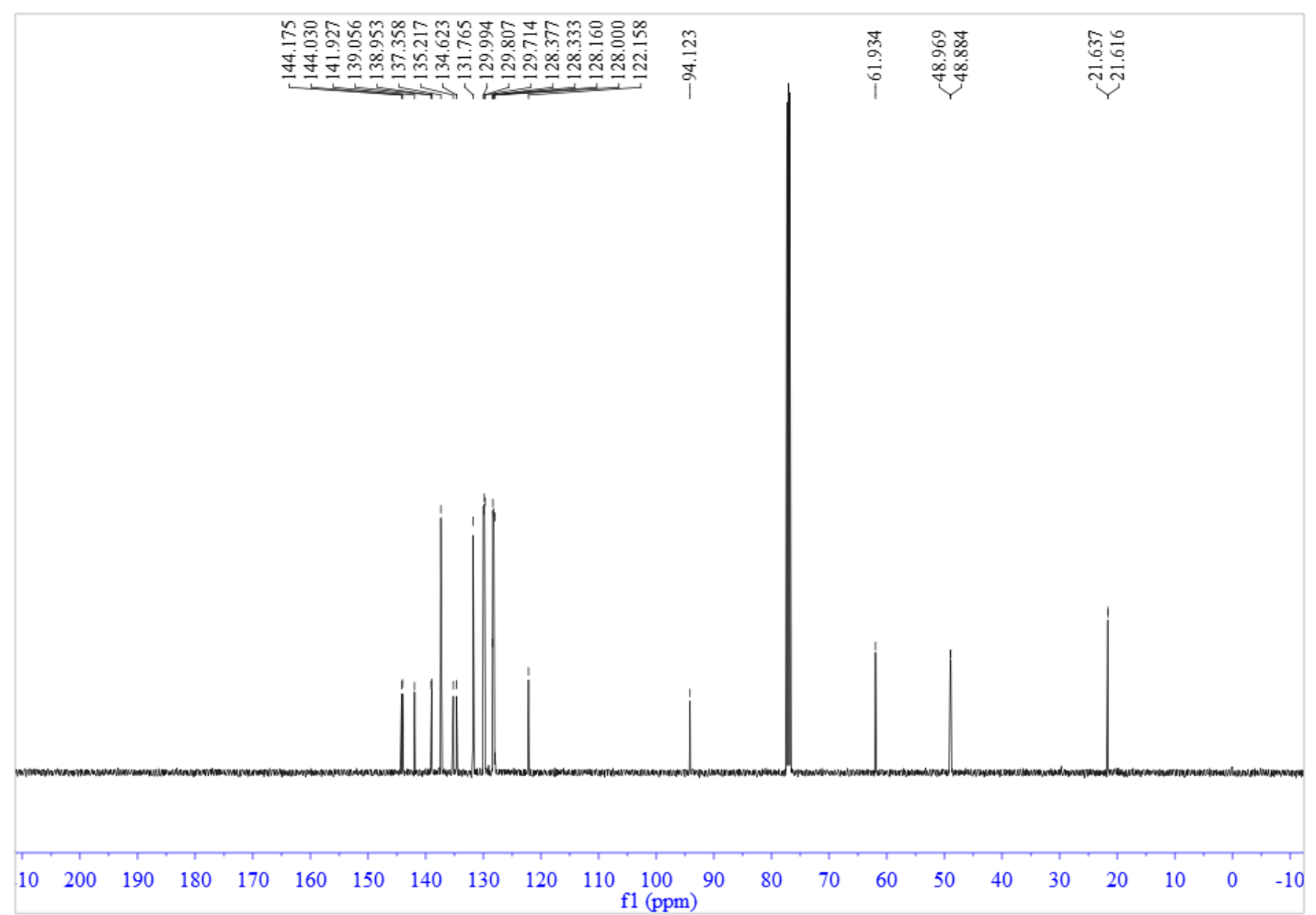


${ }^{1} \mathrm{H}$ NMR $\left(500 \mathrm{MHz}, \mathrm{CDCl}_{3}\right.$ ) of compound $\mathbf{3 k}$

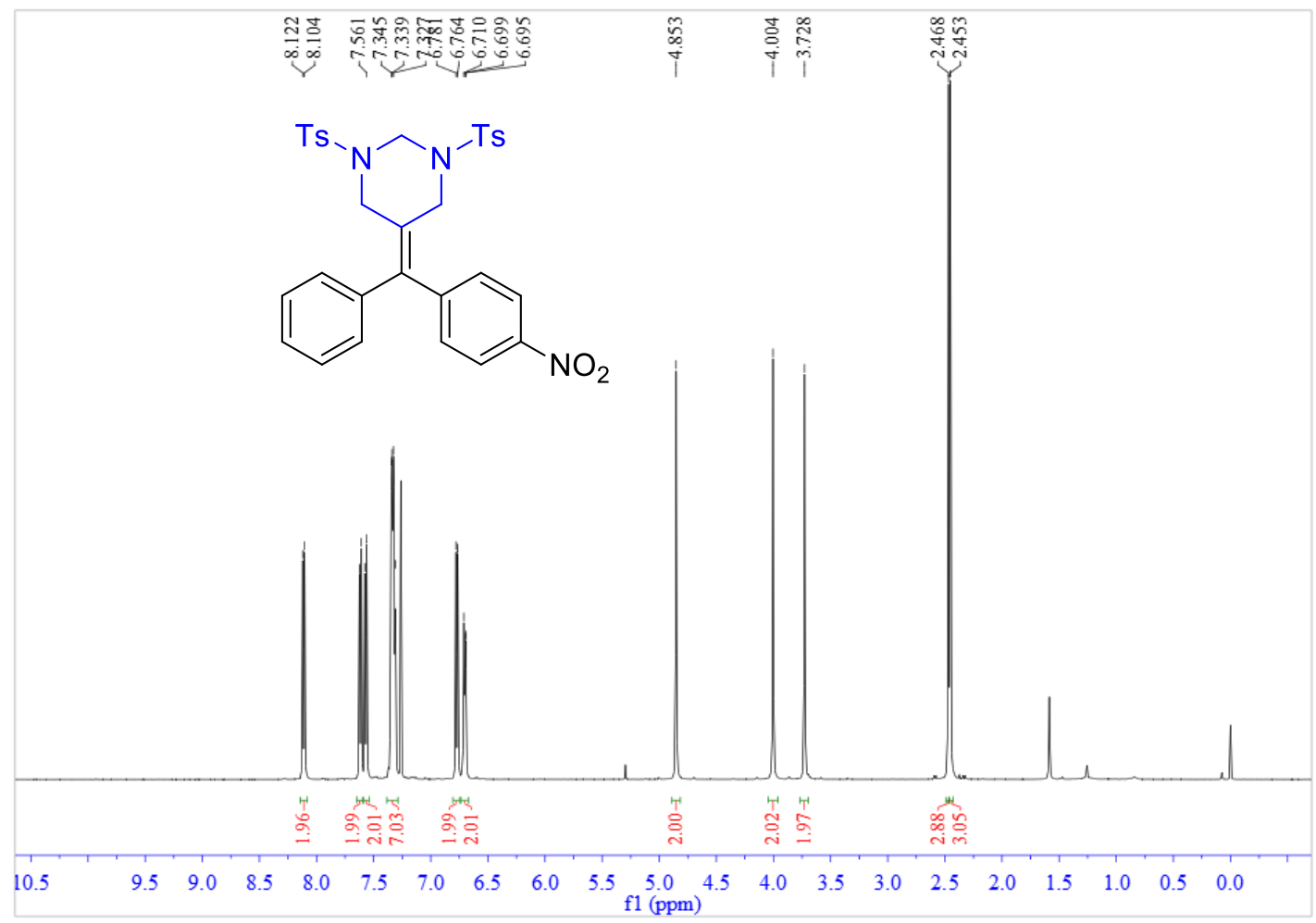

${ }^{13} \mathrm{C}$ NMR (125 MHz, $\left.\mathrm{CDCl}_{3}\right)$ of compound 3k

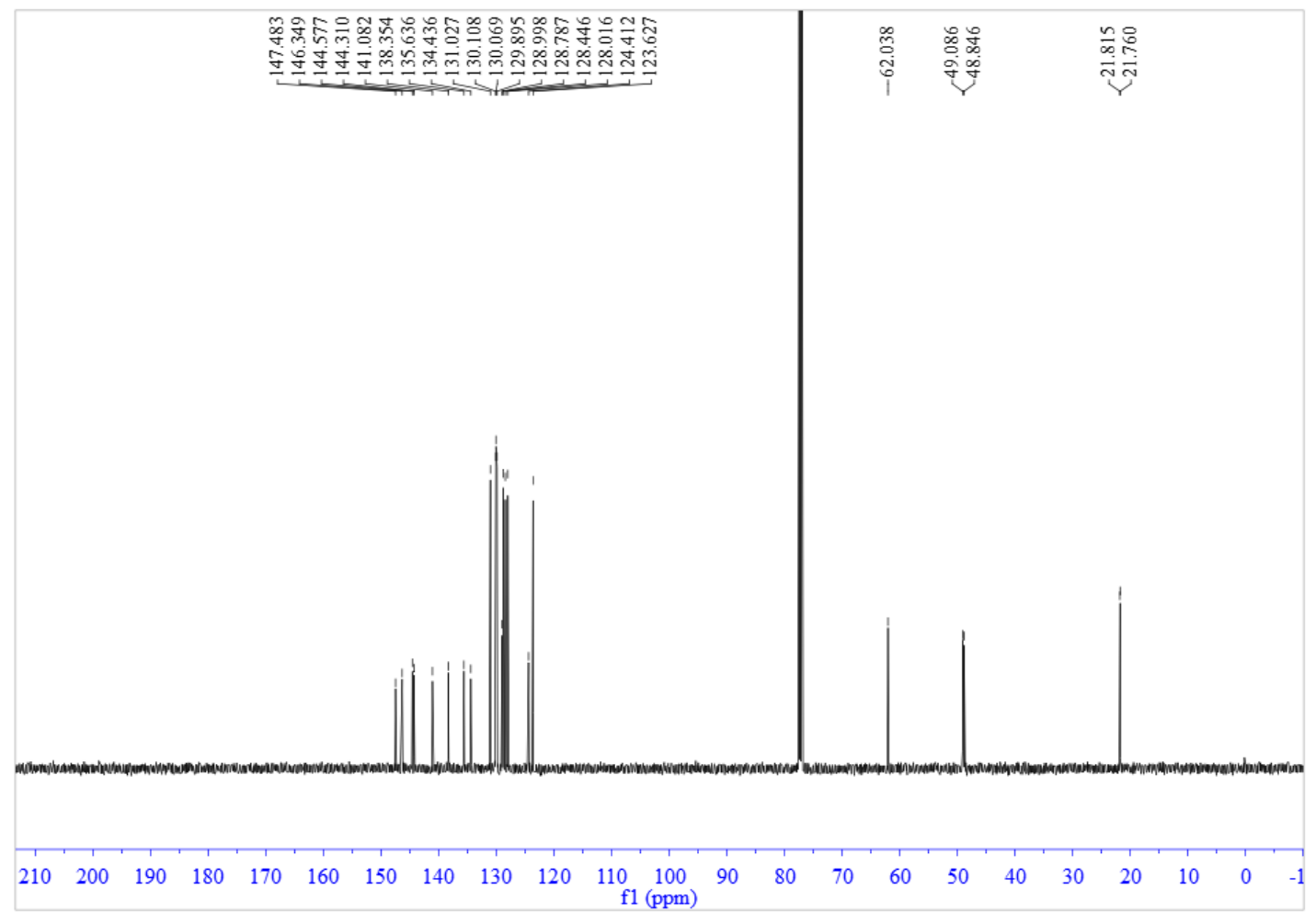


${ }^{1} \mathrm{H}$ NMR (500 MHz, $\mathrm{CDCl}_{3}$ ) of compound 31

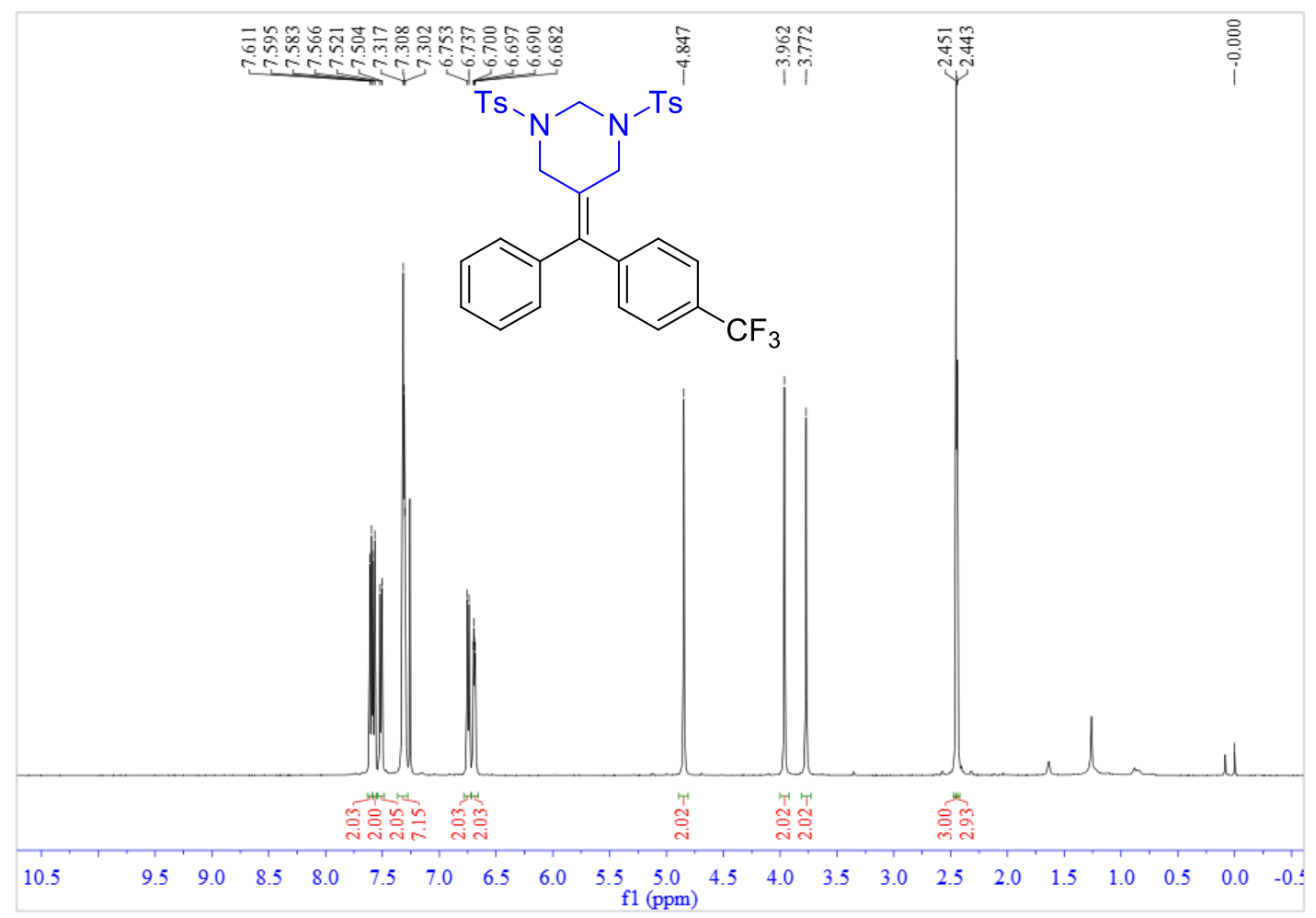

${ }^{13} \mathrm{C}$ NMR (125 MHz, $\mathrm{CDCl}_{3}$ ) of compound $3 \mathrm{I}$

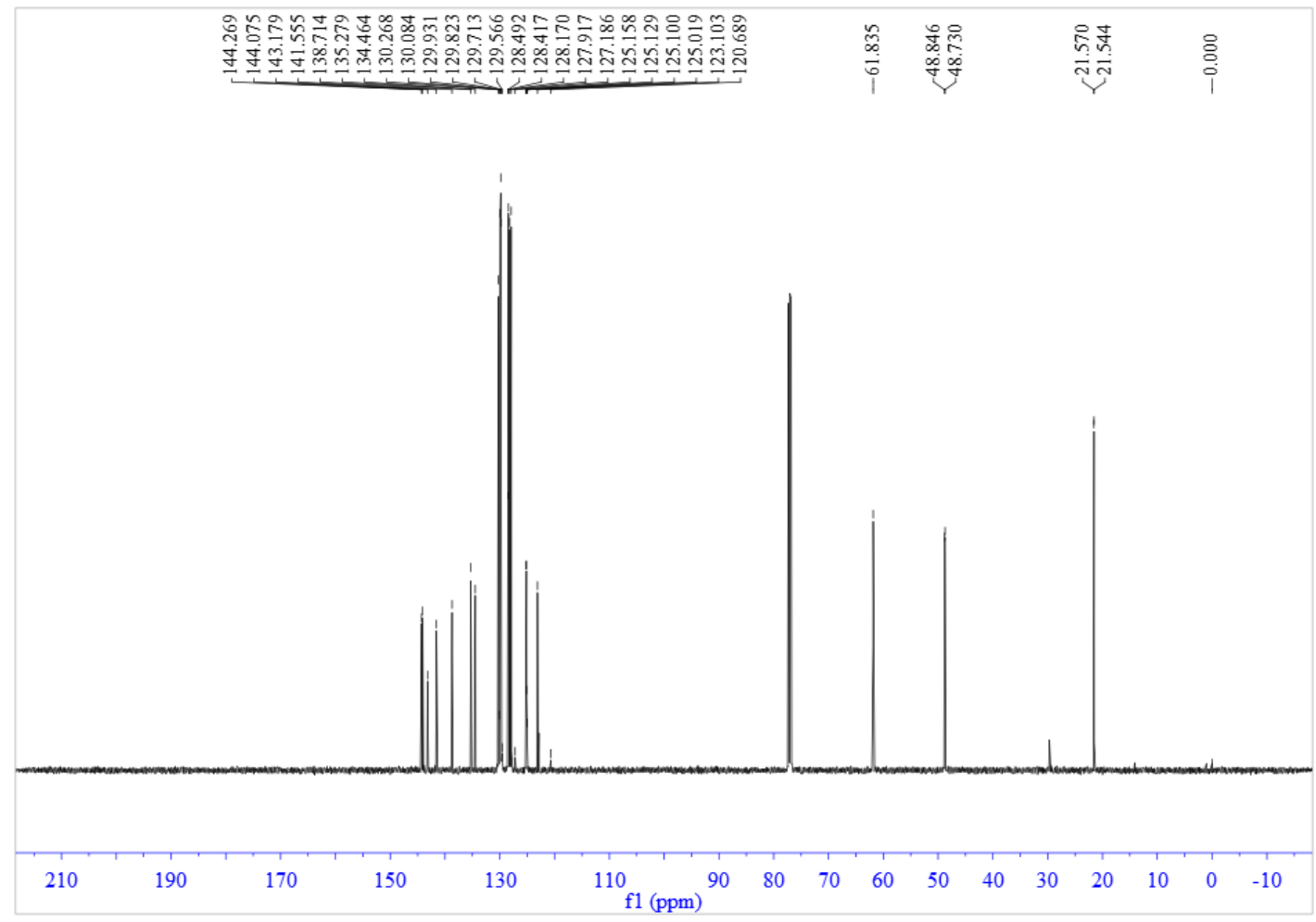


${ }^{19} \mathrm{~F}$ NMR $\left(470 \mathrm{MHz}, \mathrm{CDCl}_{3}\right)$ of compound $3 \mathbf{I}$

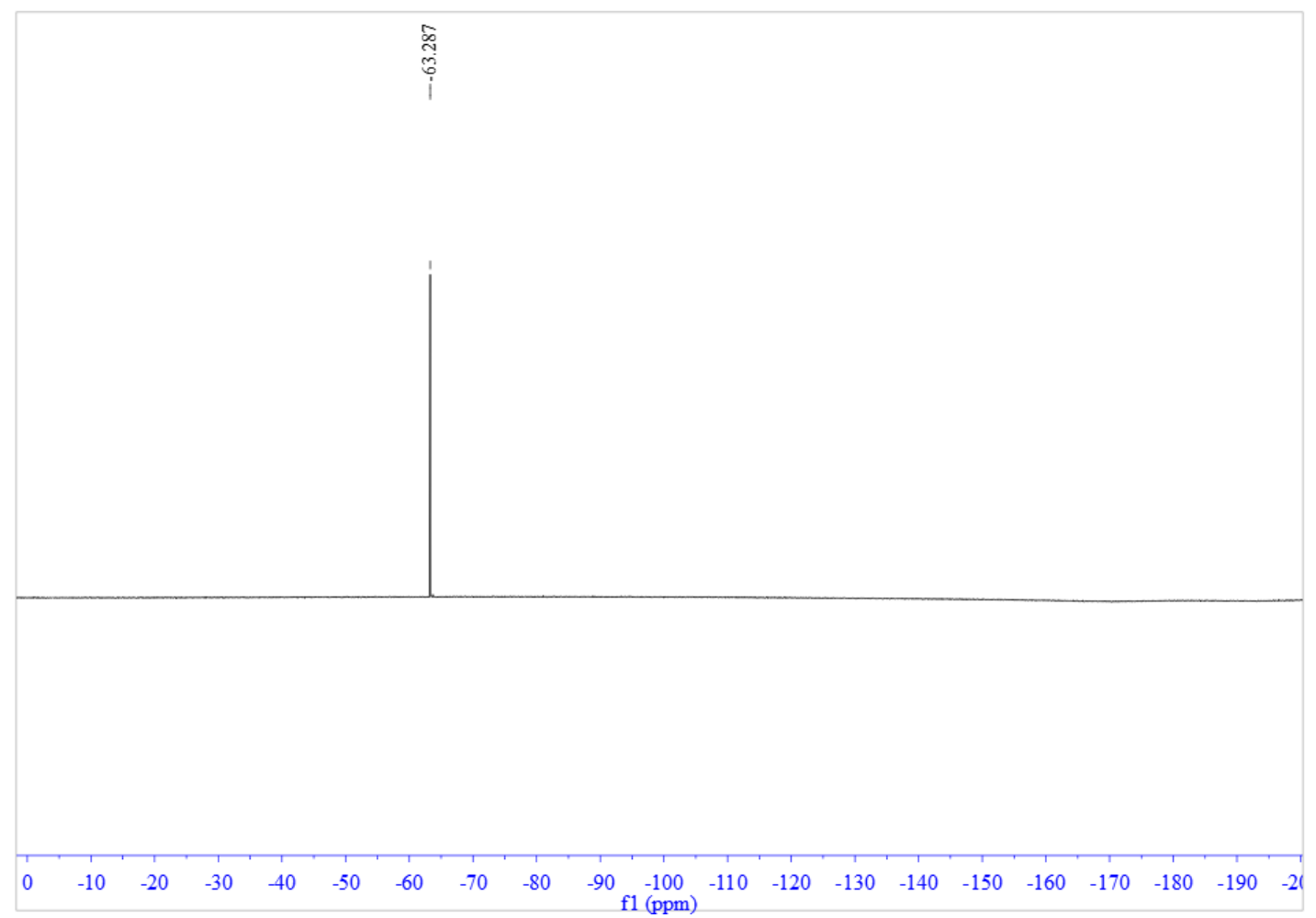


${ }^{1} \mathrm{H}$ NMR (500 MHz, $\mathrm{CDCl}_{3}$ ) of compound $\mathbf{3 m}$

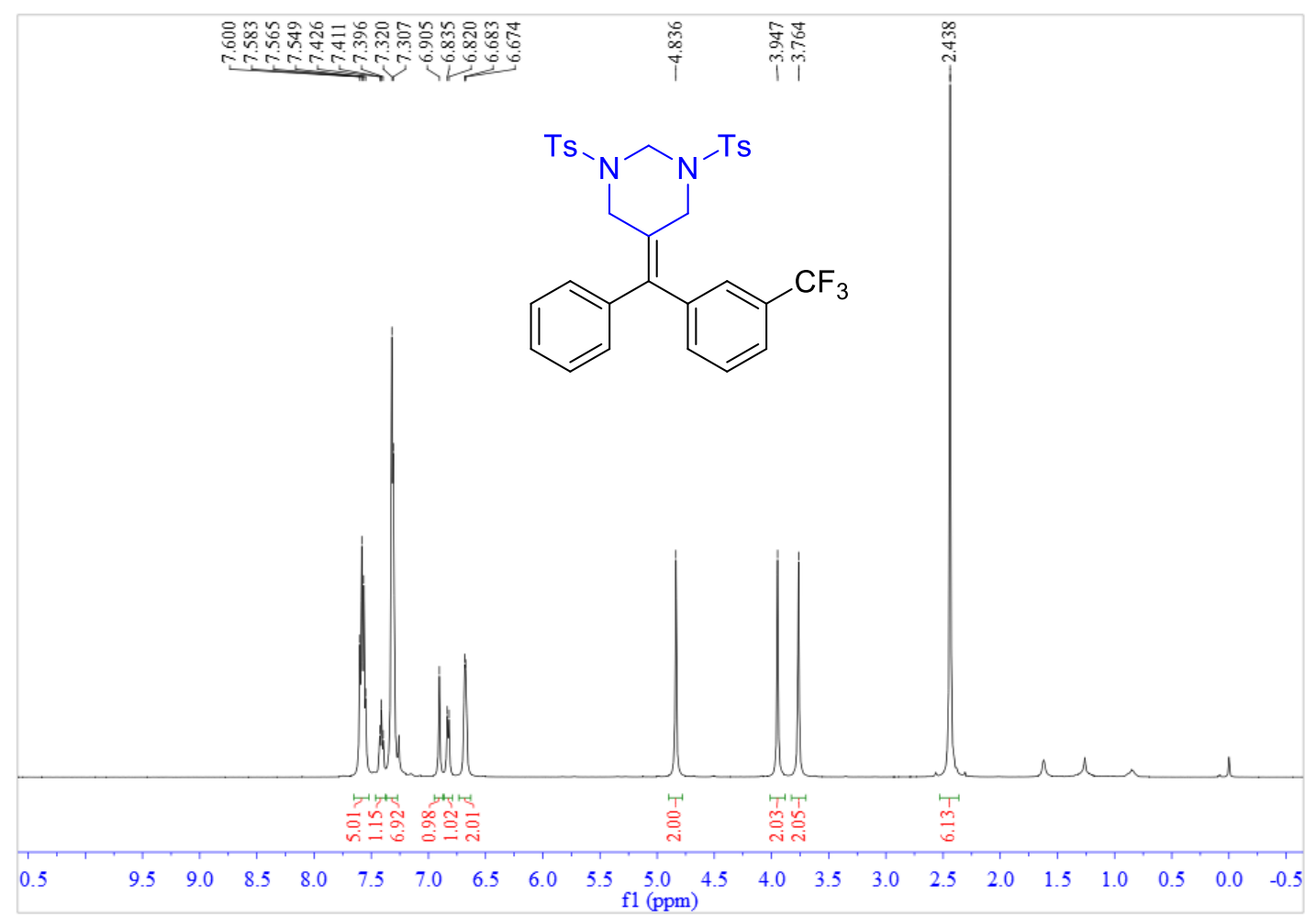

${ }^{13} \mathrm{C}$ NMR (125 MHz, $\mathrm{CDCl}_{3}$ ) of compound $\mathbf{3 m}$

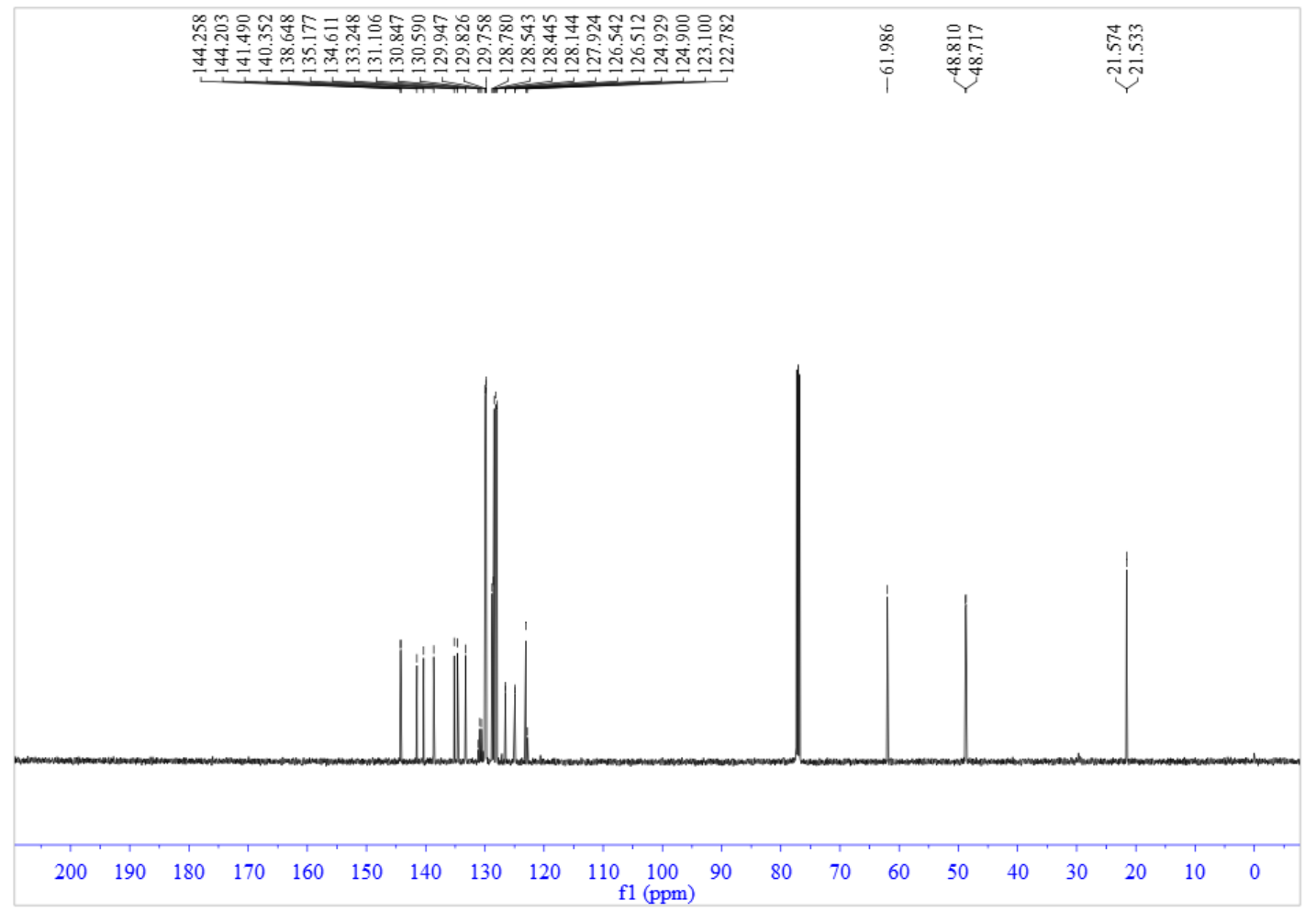


${ }^{19} \mathrm{~F}$ NMR $\left(470 \mathrm{MHz}, \mathrm{CDCl}_{3}\right.$ ) of compound $3 \mathbf{m}$

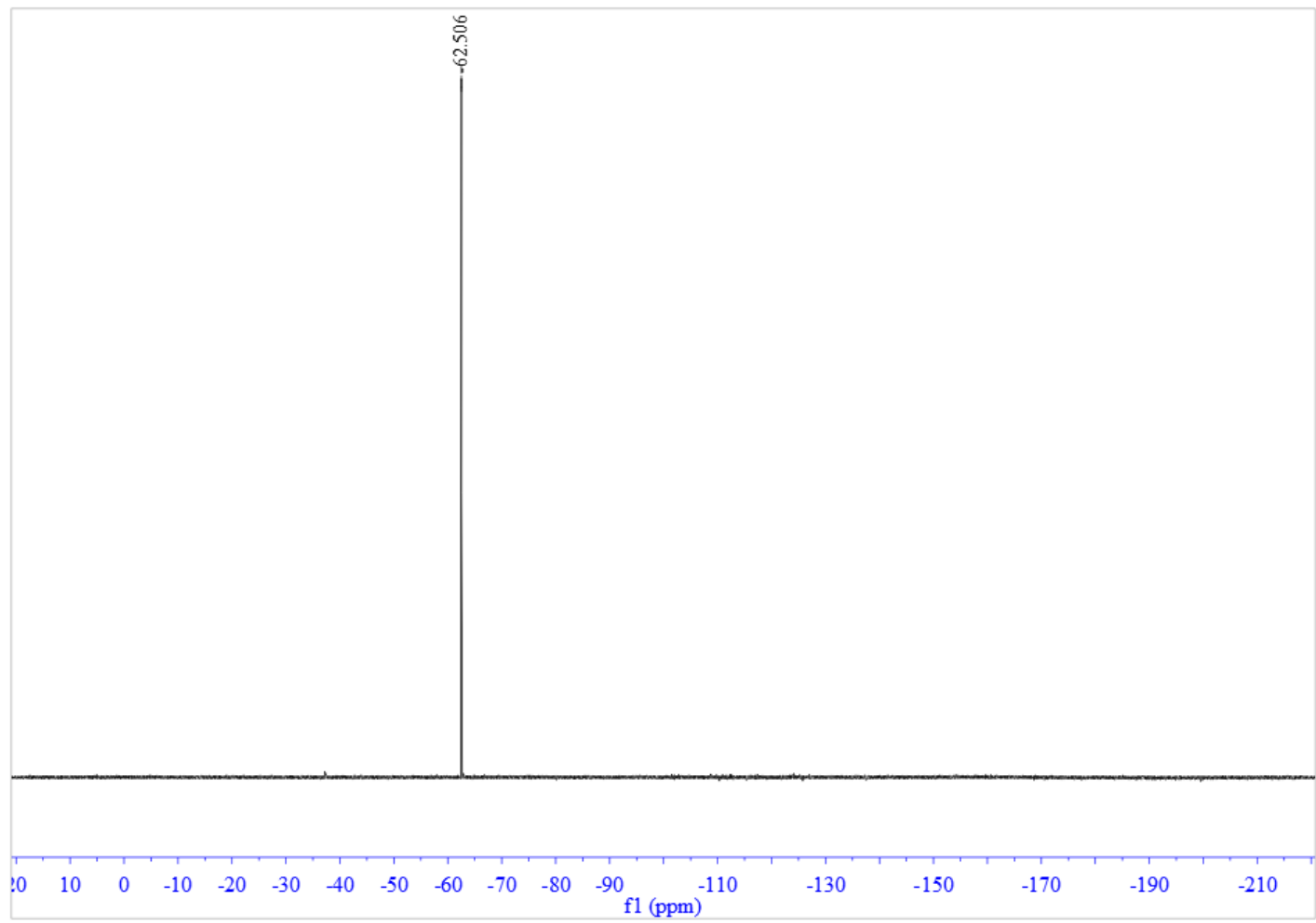


${ }^{1} \mathrm{H}$ NMR $\left(500 \mathrm{MHz}, \mathrm{CDCl}_{3}\right)$ of compound $\mathbf{3 n}$

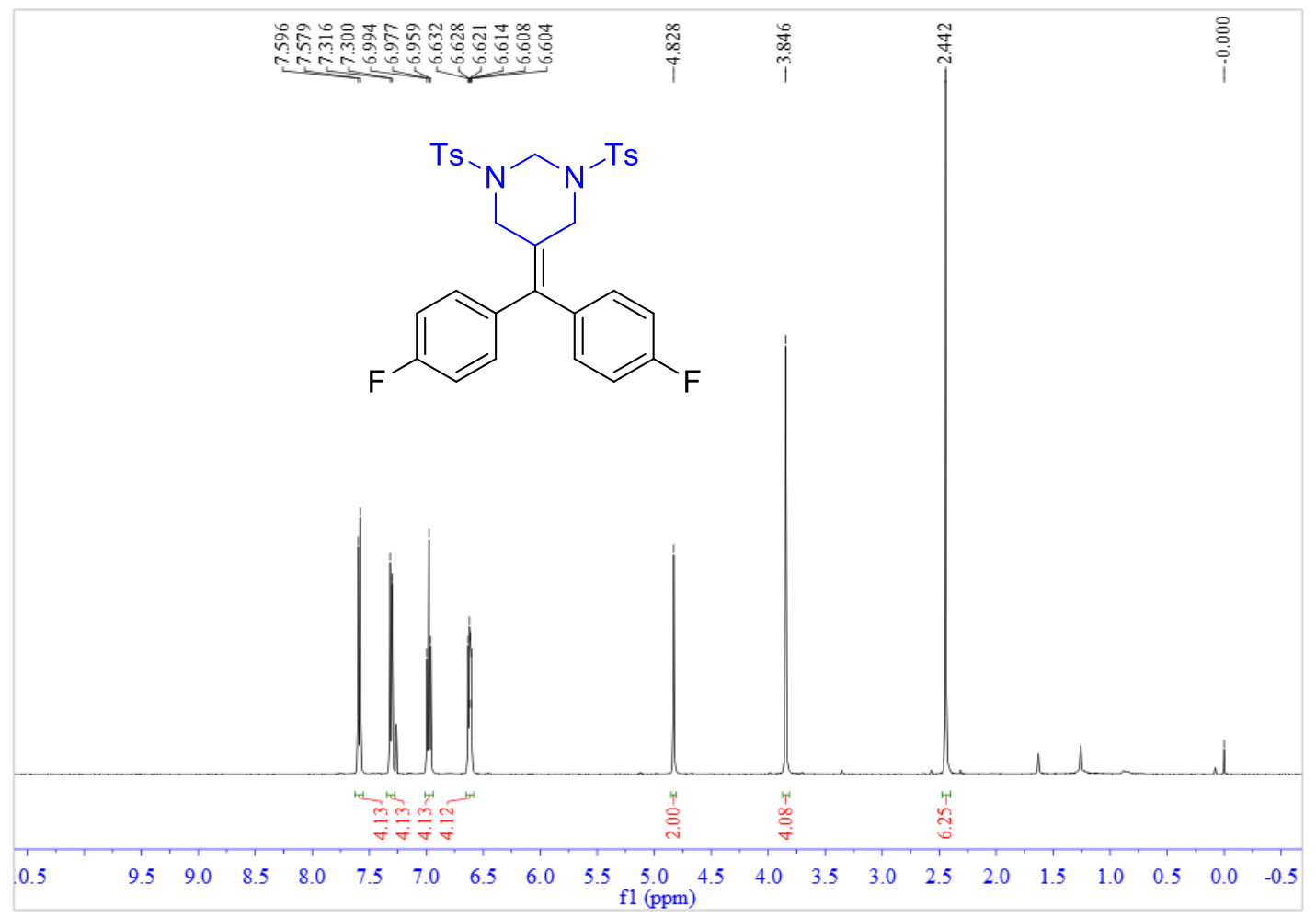

${ }^{13} \mathrm{C}$ NMR (125 MHz, $\mathrm{CDCl}_{3}$ ) of compound $3 \mathbf{n}$

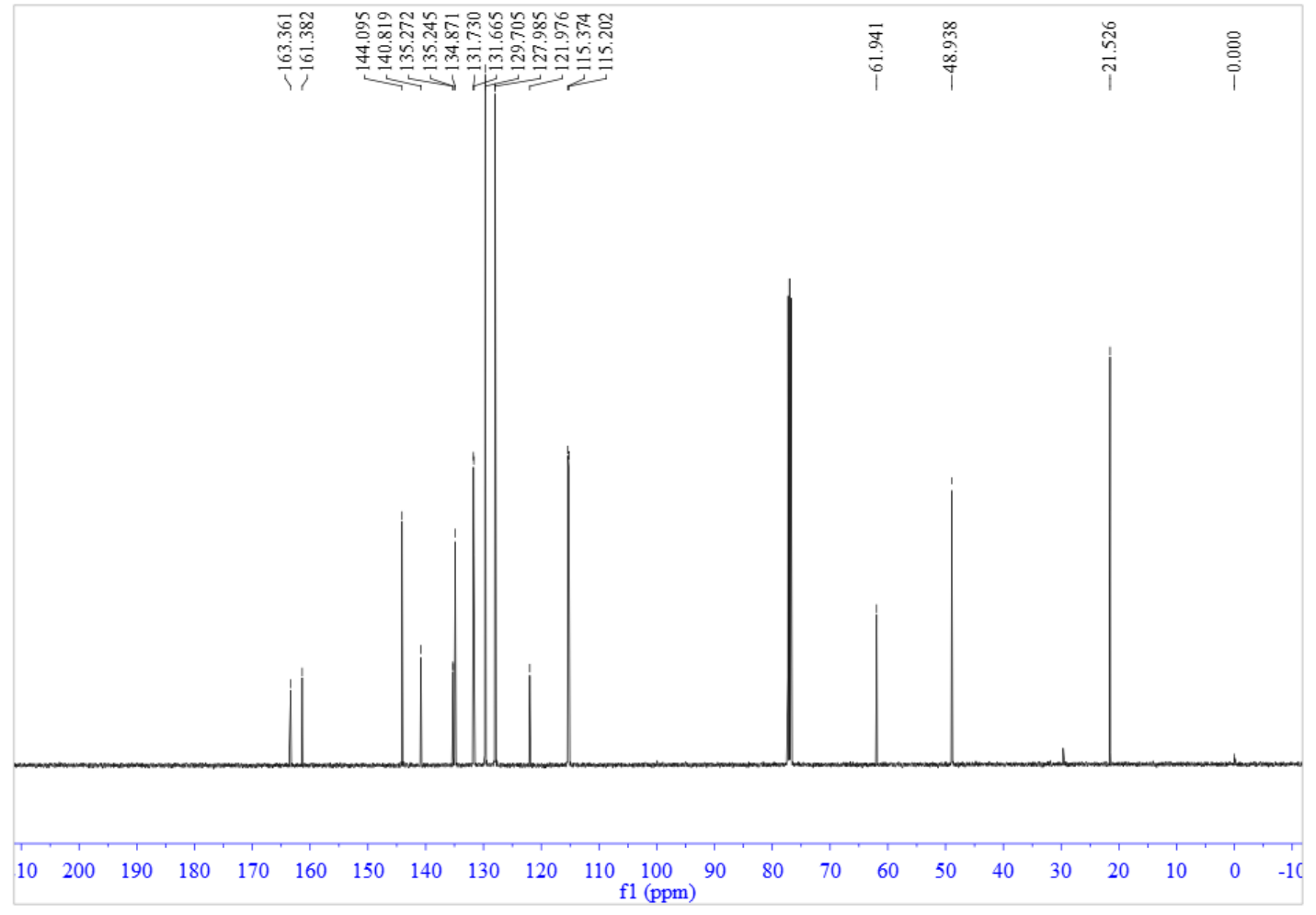


${ }^{19} \mathrm{~F}$ NMR (470 MHz, $\mathrm{CDCl}_{3}$ ) of compound 3n

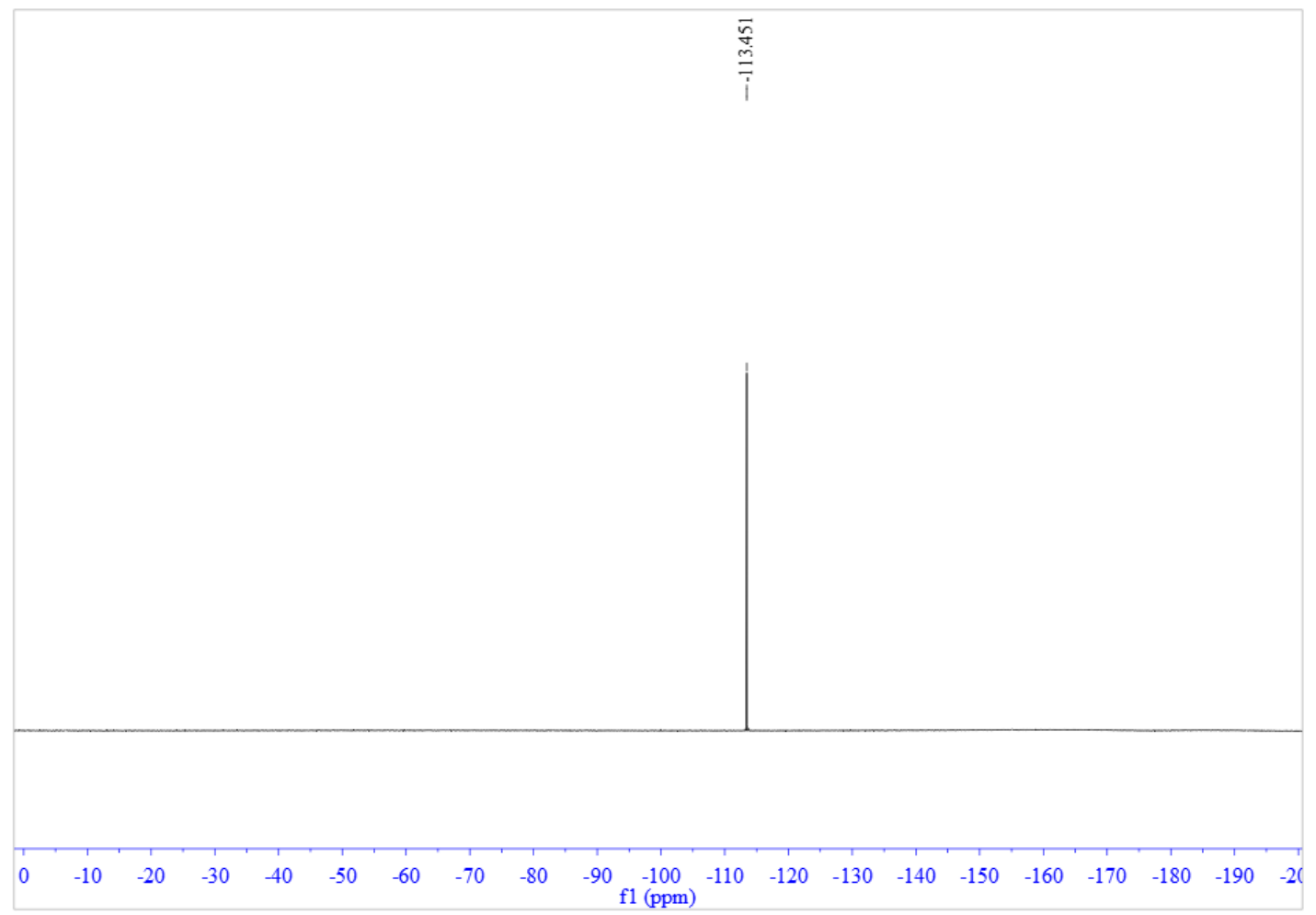


${ }^{1} \mathrm{H}$ NMR $\left(500 \mathrm{MHz}, \mathrm{CDCl}_{3}\right.$ ) of compound 30

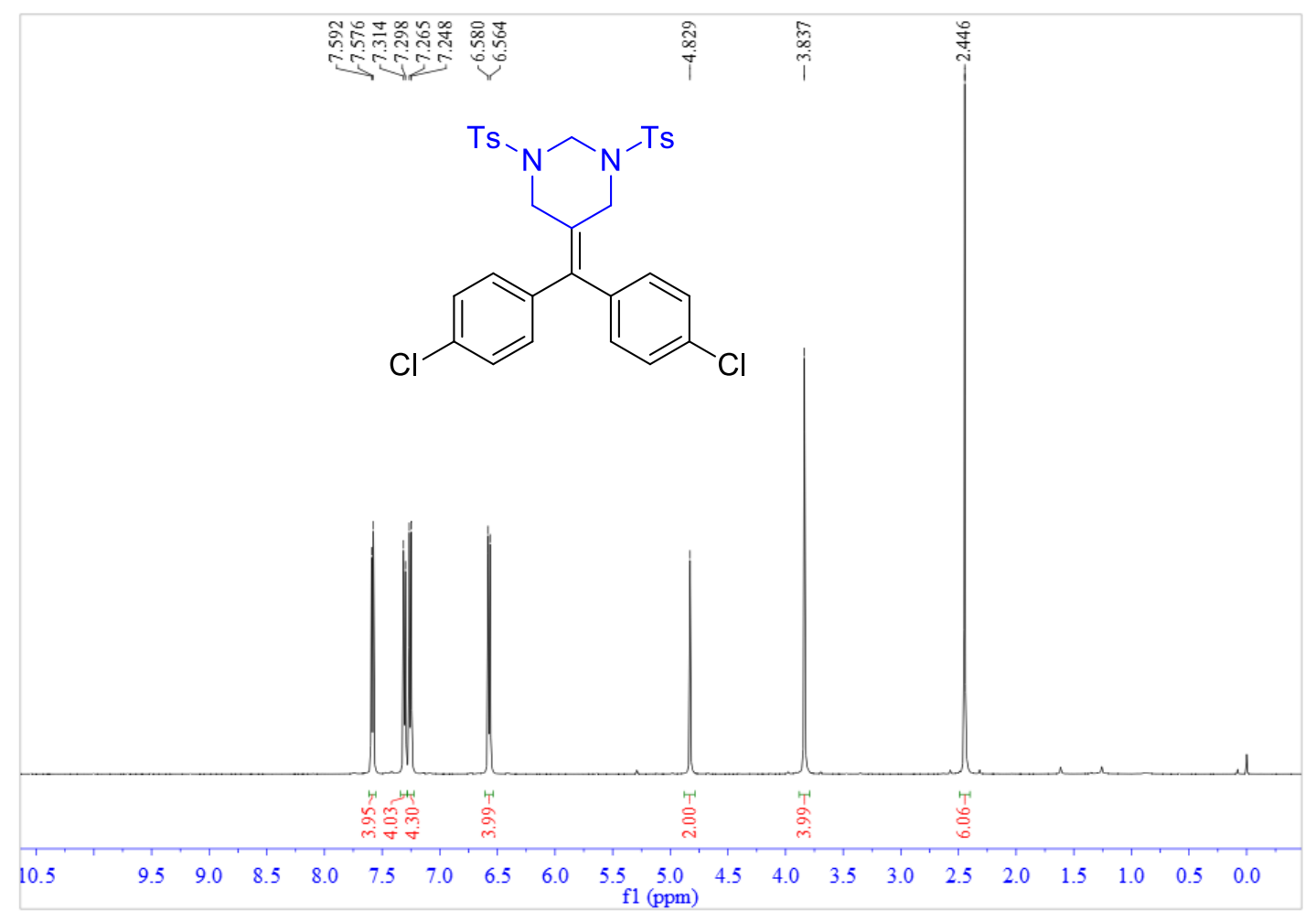

${ }^{13} \mathrm{C}$ NMR $\left(125 \mathrm{MHz}, \mathrm{CDCl}_{3}\right)$ of compound $3 \mathrm{o}$

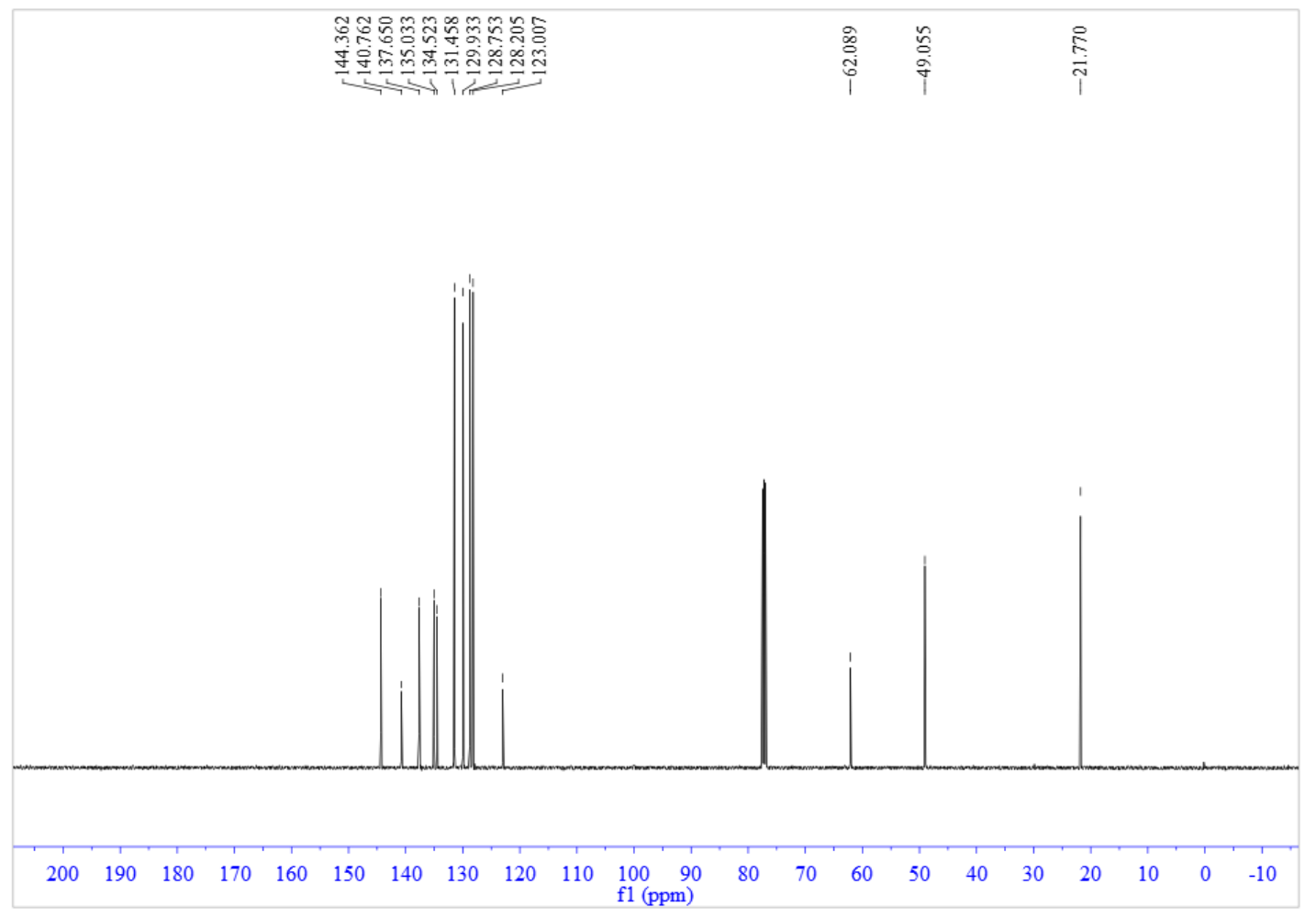




\section{${ }^{1} \mathrm{H}$ NMR $\left(500 \mathrm{MHz}, \mathrm{CDCl}_{3}\right)$ of compound 3p}

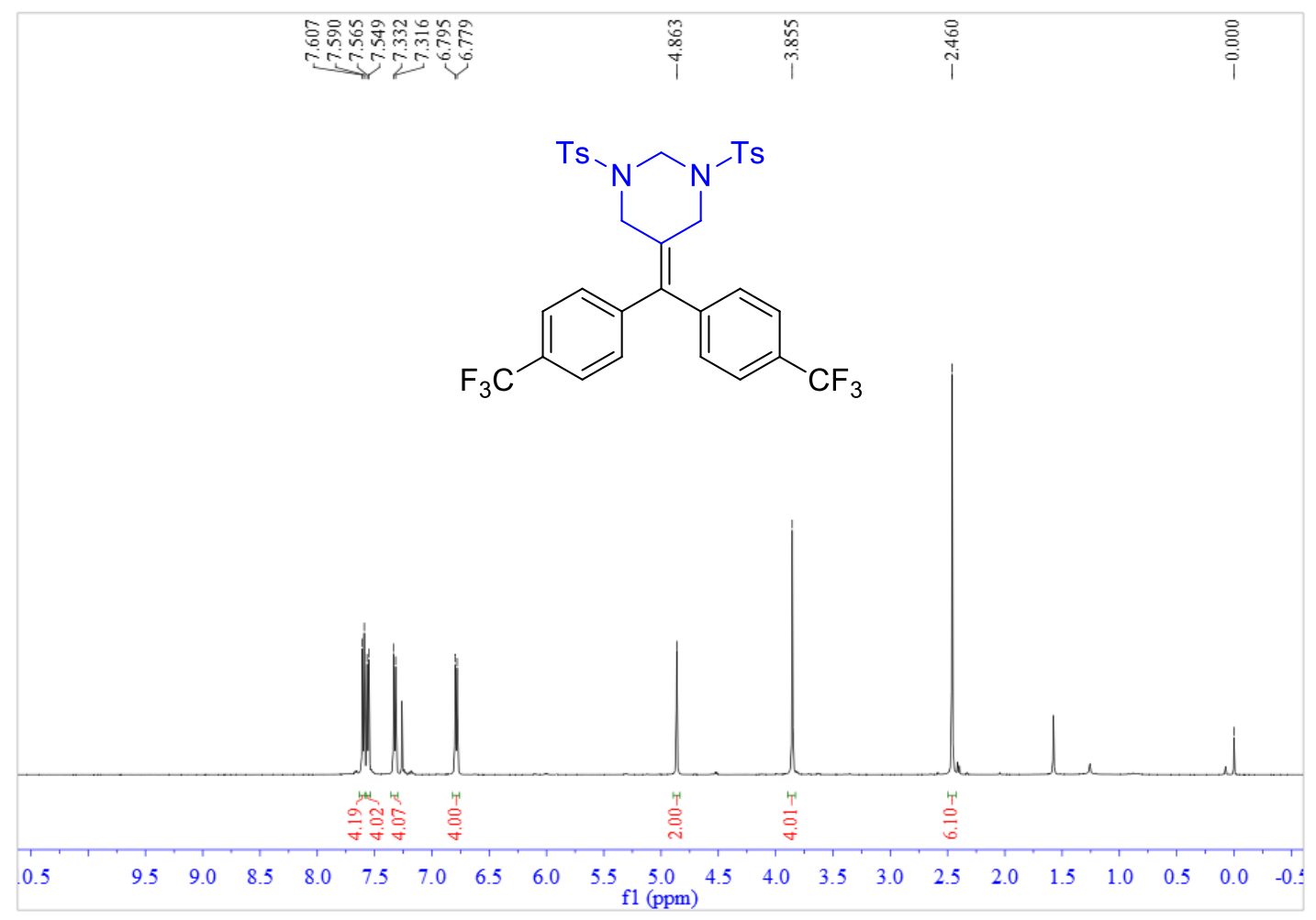

${ }^{13} \mathrm{C}$ NMR $\left(125 \mathrm{MHz}, \mathrm{CDCl}_{3}\right)$ of compound $\mathbf{3 p}$

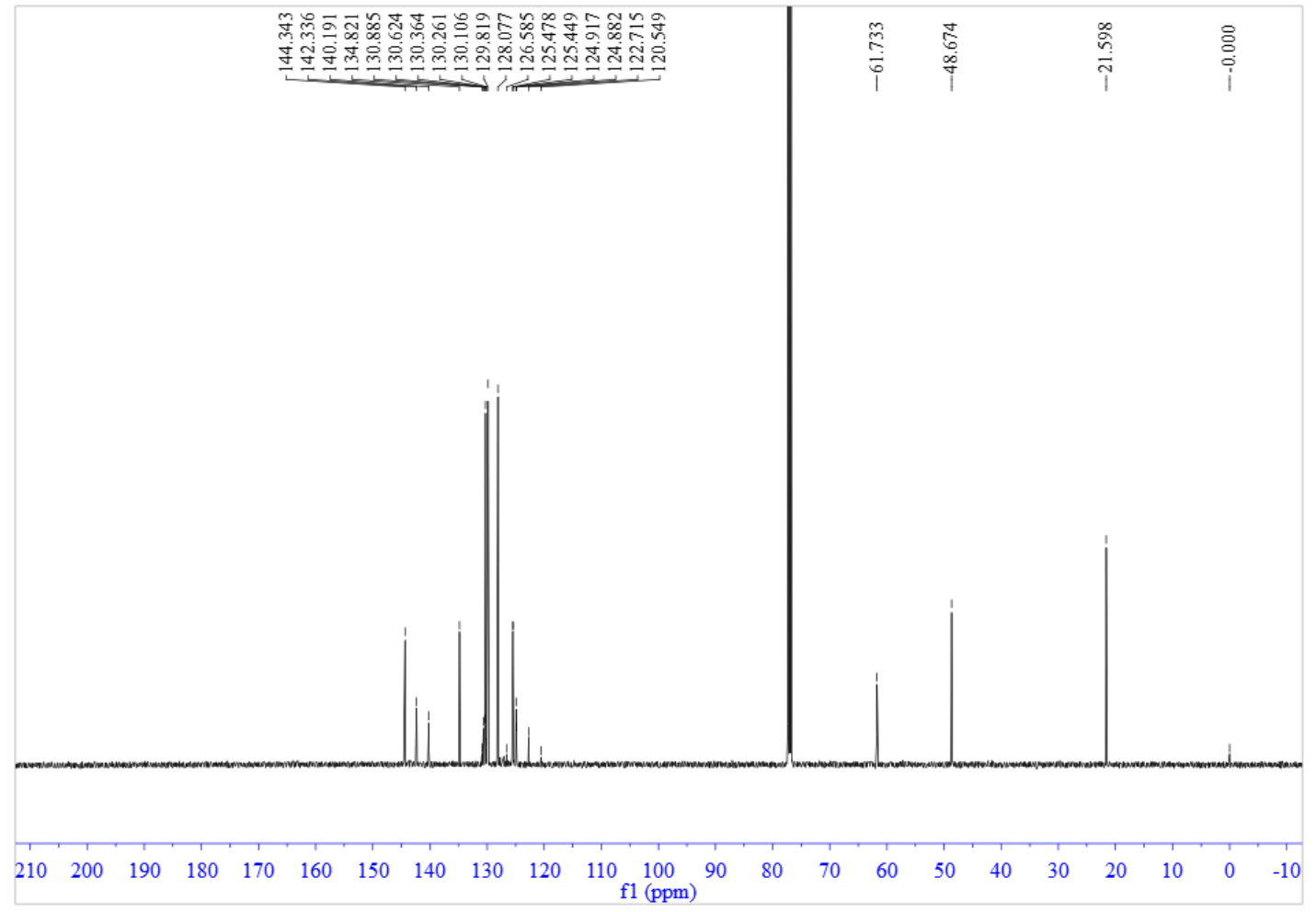


${ }^{19} \mathrm{~F}$ NMR (470 MHz, $\mathrm{CDCl}_{3}$ ) of compound 3p

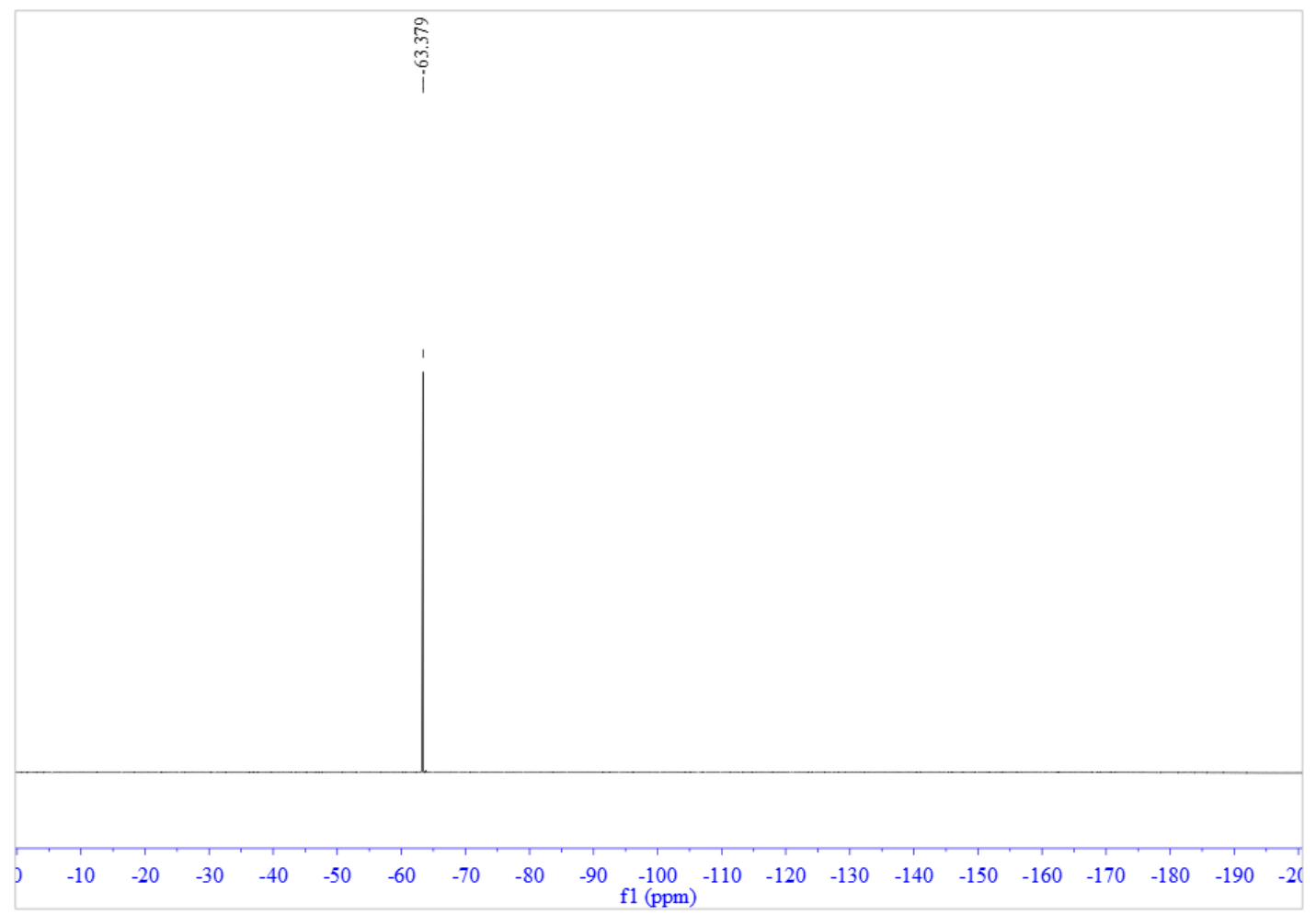


${ }^{1} \mathrm{H}$ NMR $\left(500 \mathrm{MHz}, \mathrm{CDCl}_{3}\right)$ of compound $\mathbf{3 q}$

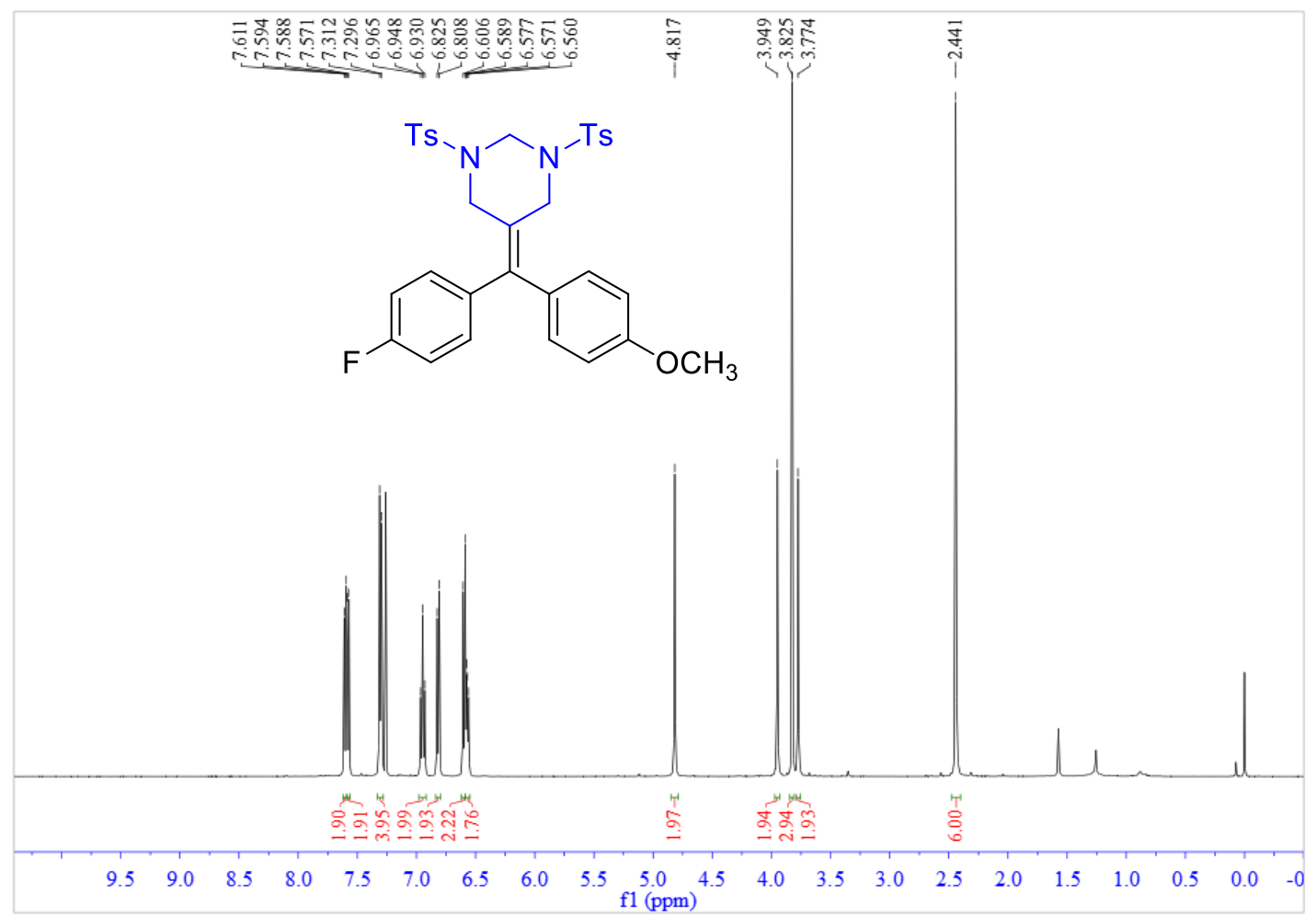

${ }^{13} \mathrm{C}$ NMR (125 MHz, $\mathrm{CDCl}_{3}$ ) of compound 3q

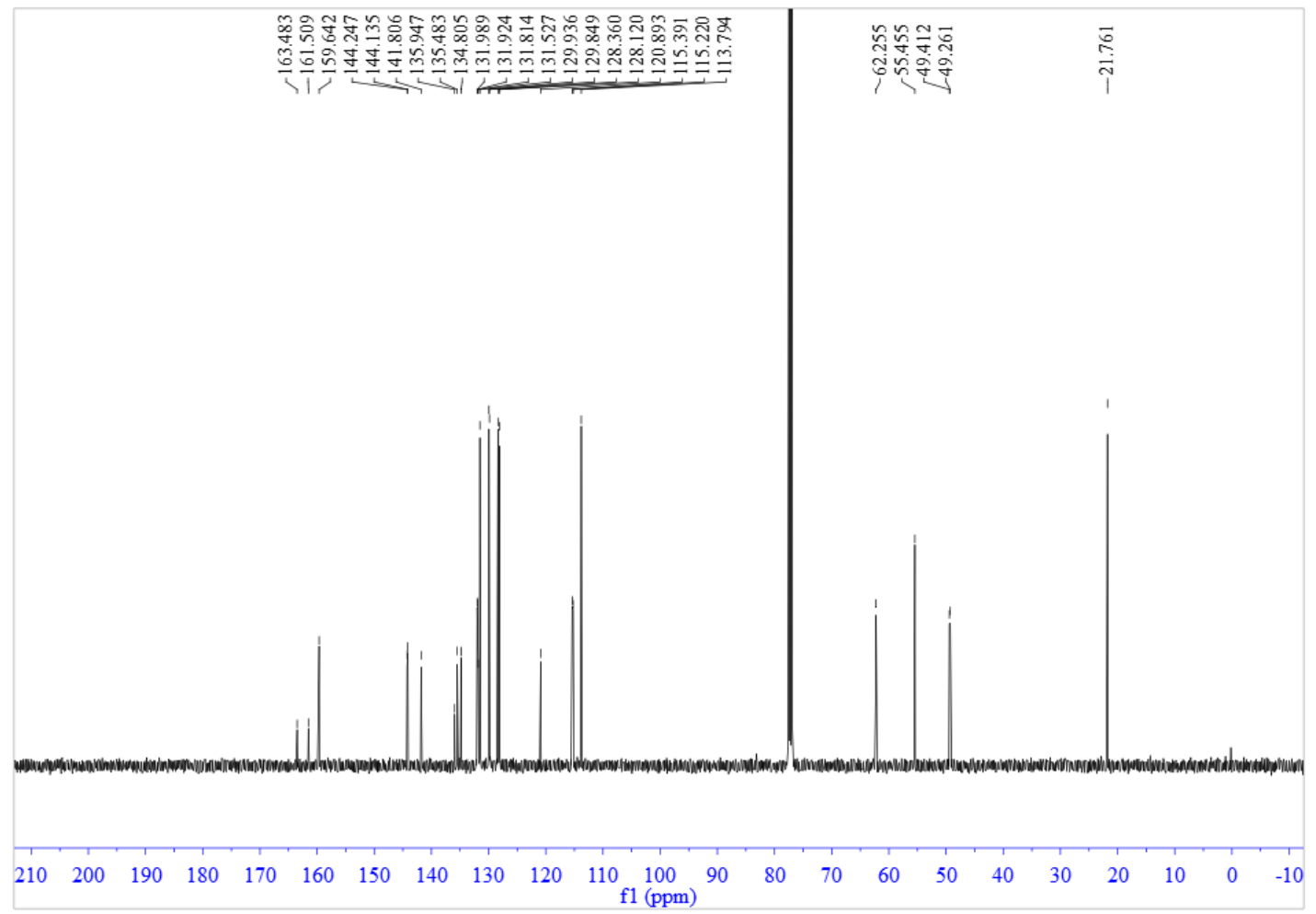


${ }^{19} \mathrm{~F}$ NMR (470 MHz, $\mathrm{CDCl}_{3}$ ) of compound $\mathbf{3 q}$

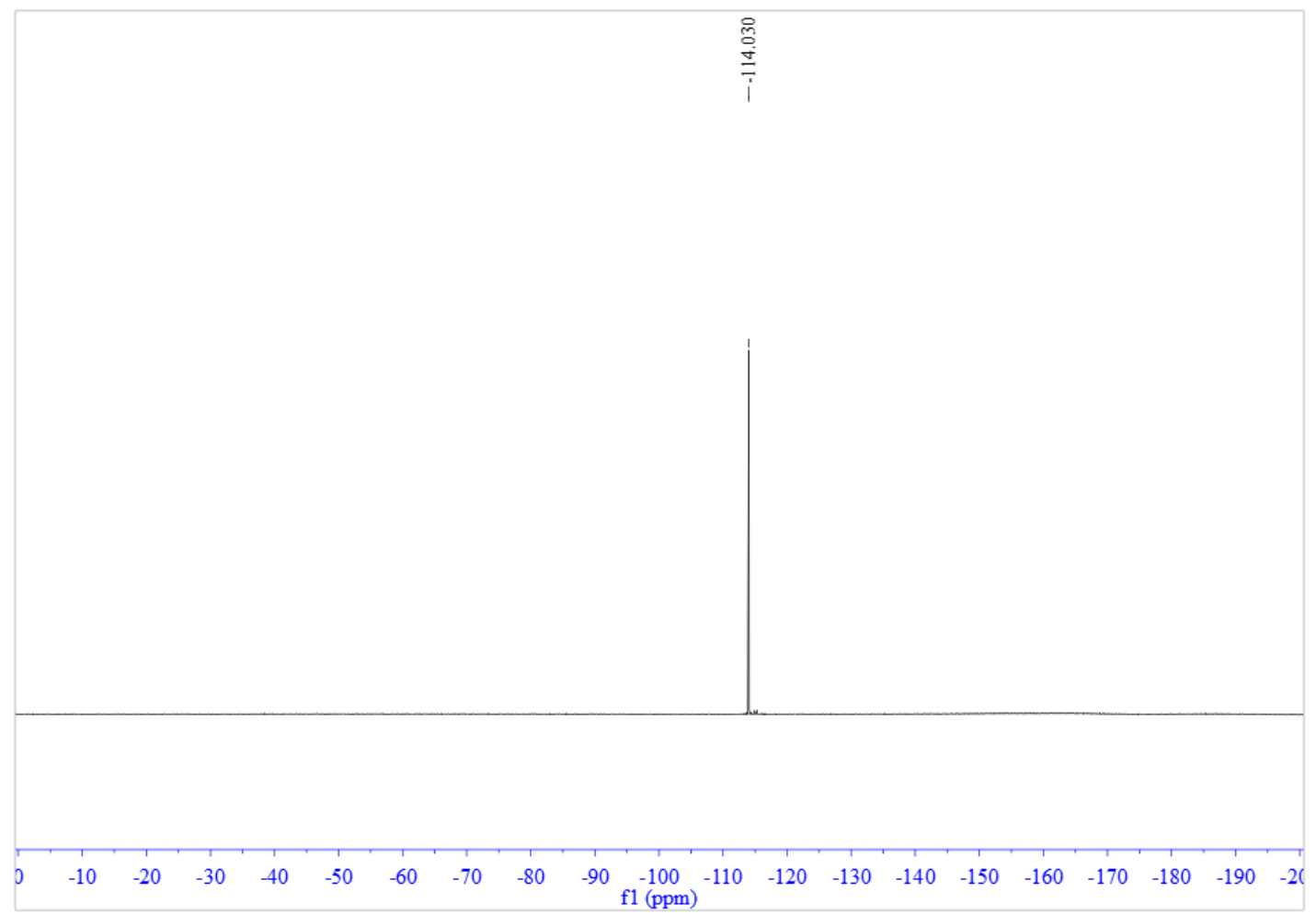


${ }^{1} \mathrm{H}$ NMR $\left(500 \mathrm{MHz}, \mathrm{CDCl}_{3}\right)$ of compound $4 \mathbf{a}$

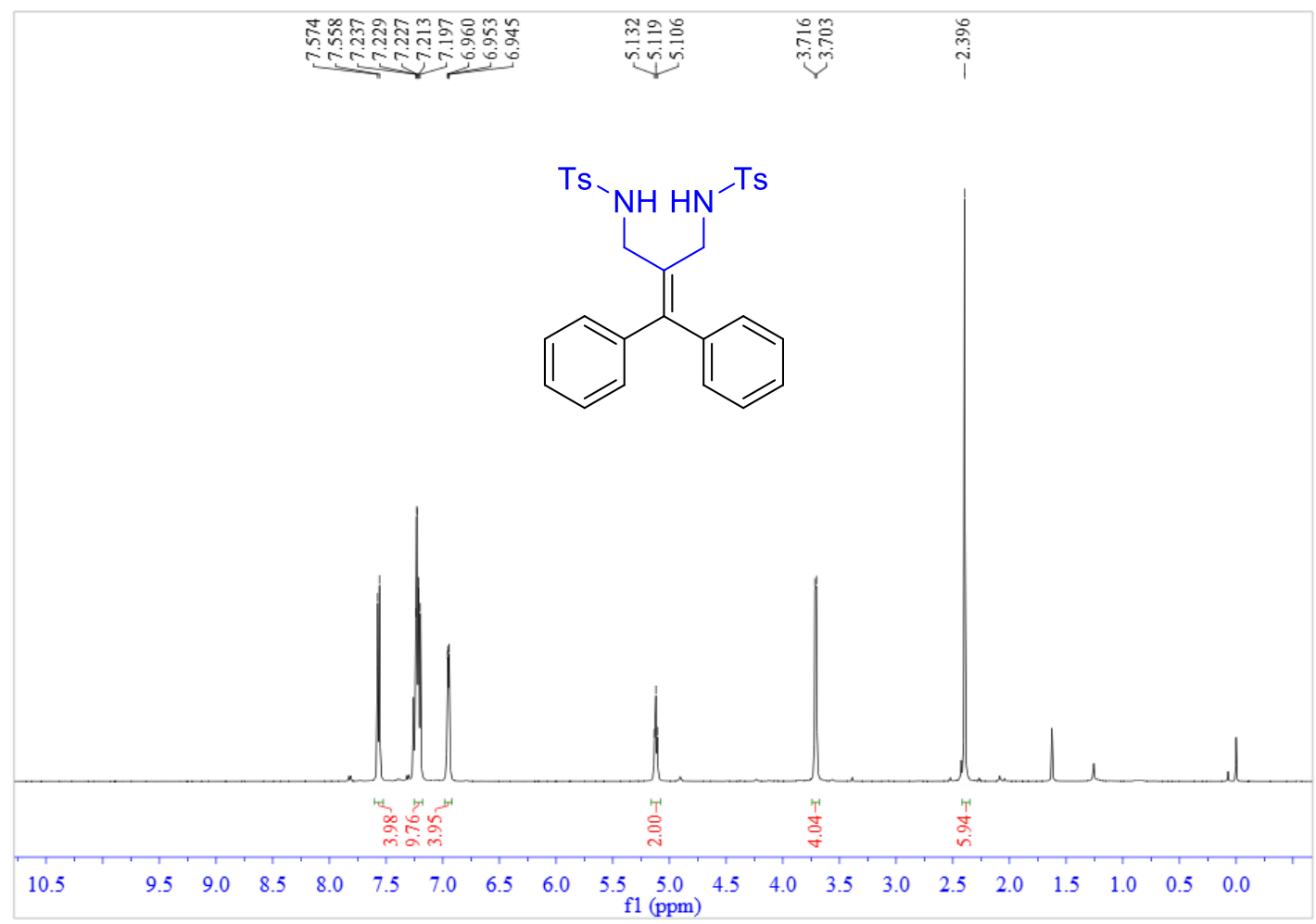

${ }^{13} \mathrm{C}$ NMR (125 MHz, $\left.\mathrm{CDCl}_{3}\right)$ of compound $4 \mathbf{a}$

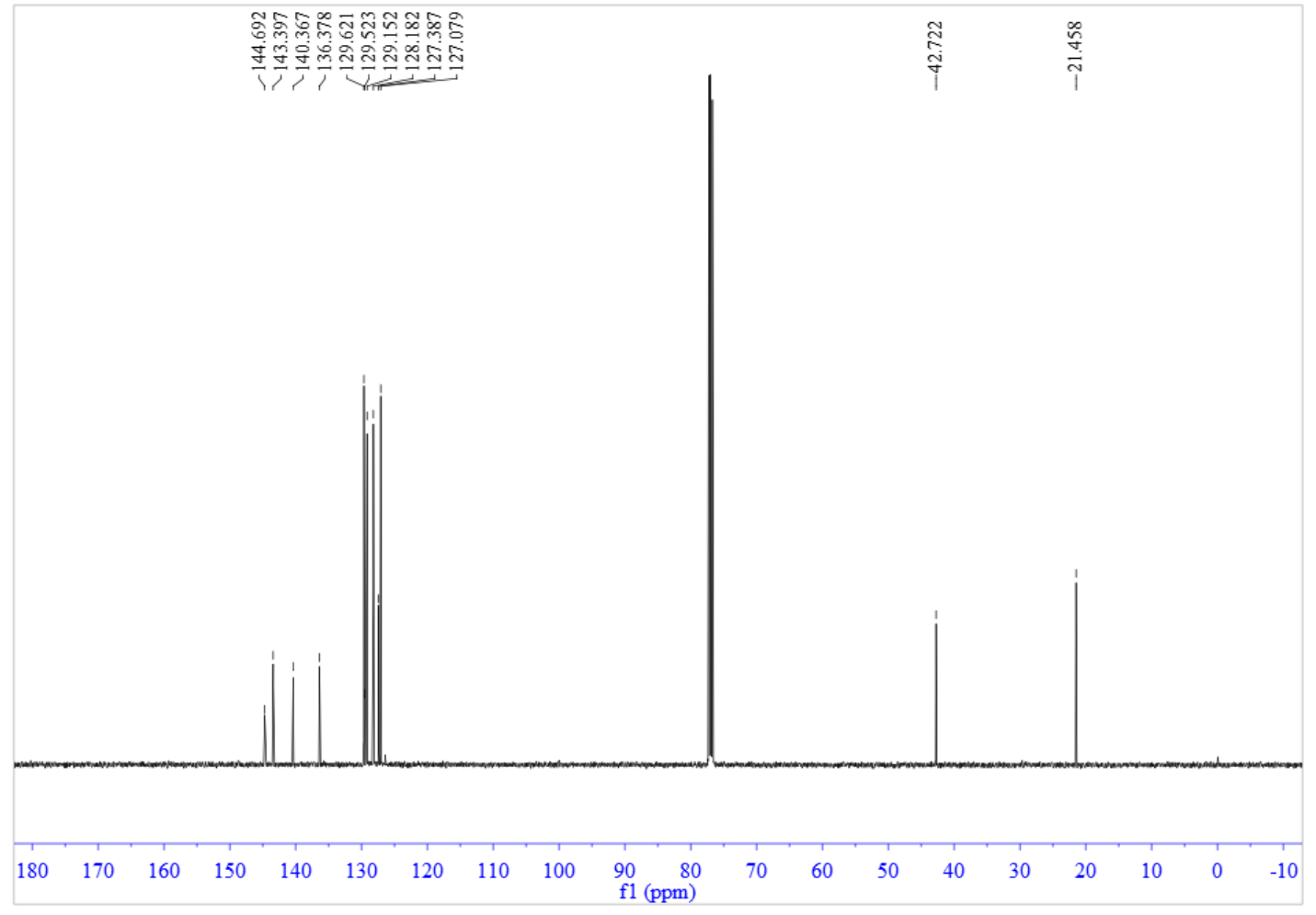




\section{${ }^{1} \mathrm{H}$ NMR $\left(500 \mathrm{MHz}, \mathrm{CDCl}_{3}\right.$ ) of compound $6 \mathbf{a}$}

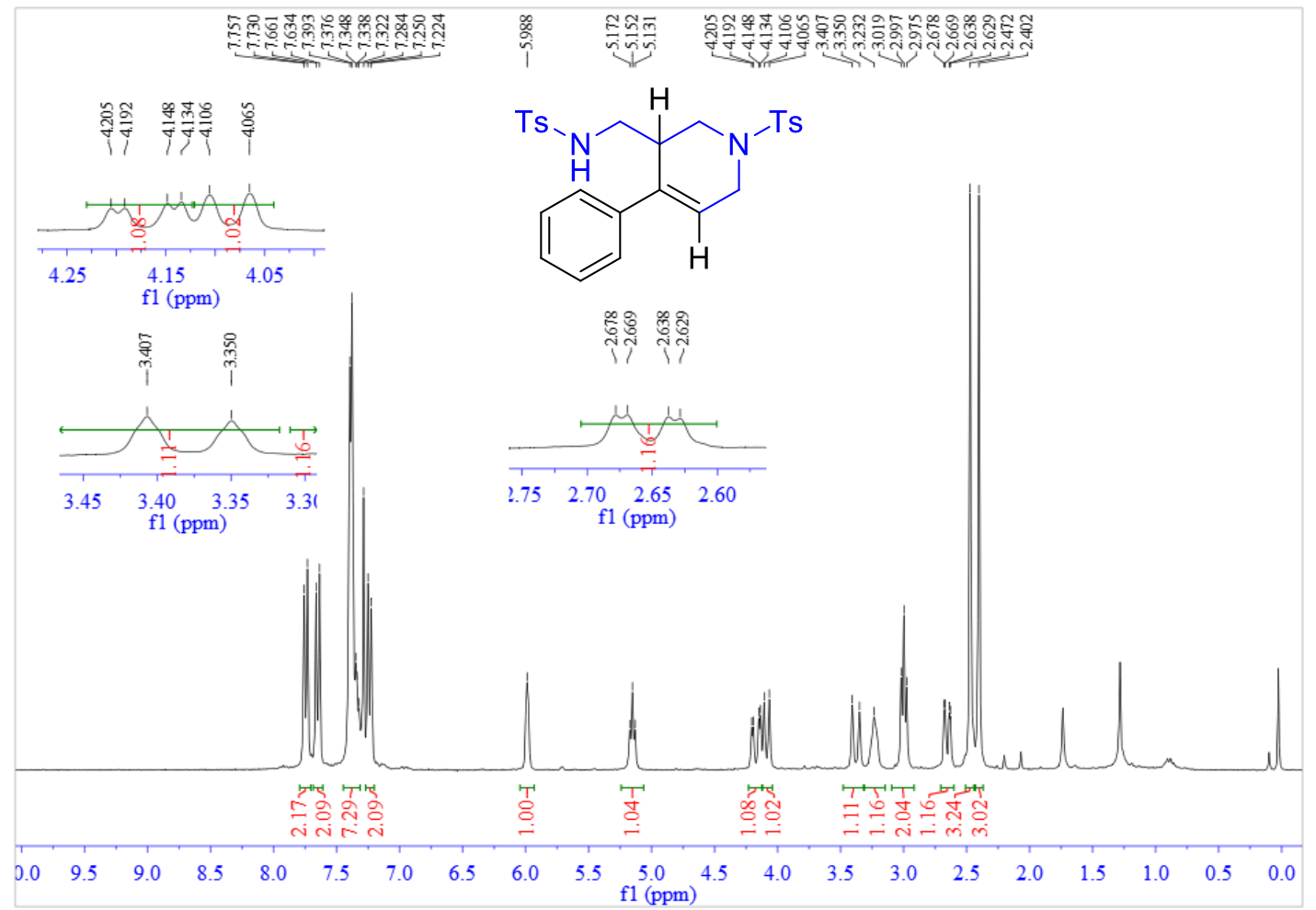

${ }^{13} \mathrm{C}$ NMR (125 MHz, $\left.\mathrm{CDCl}_{3}\right)$ of compound $6 \mathbf{a}$

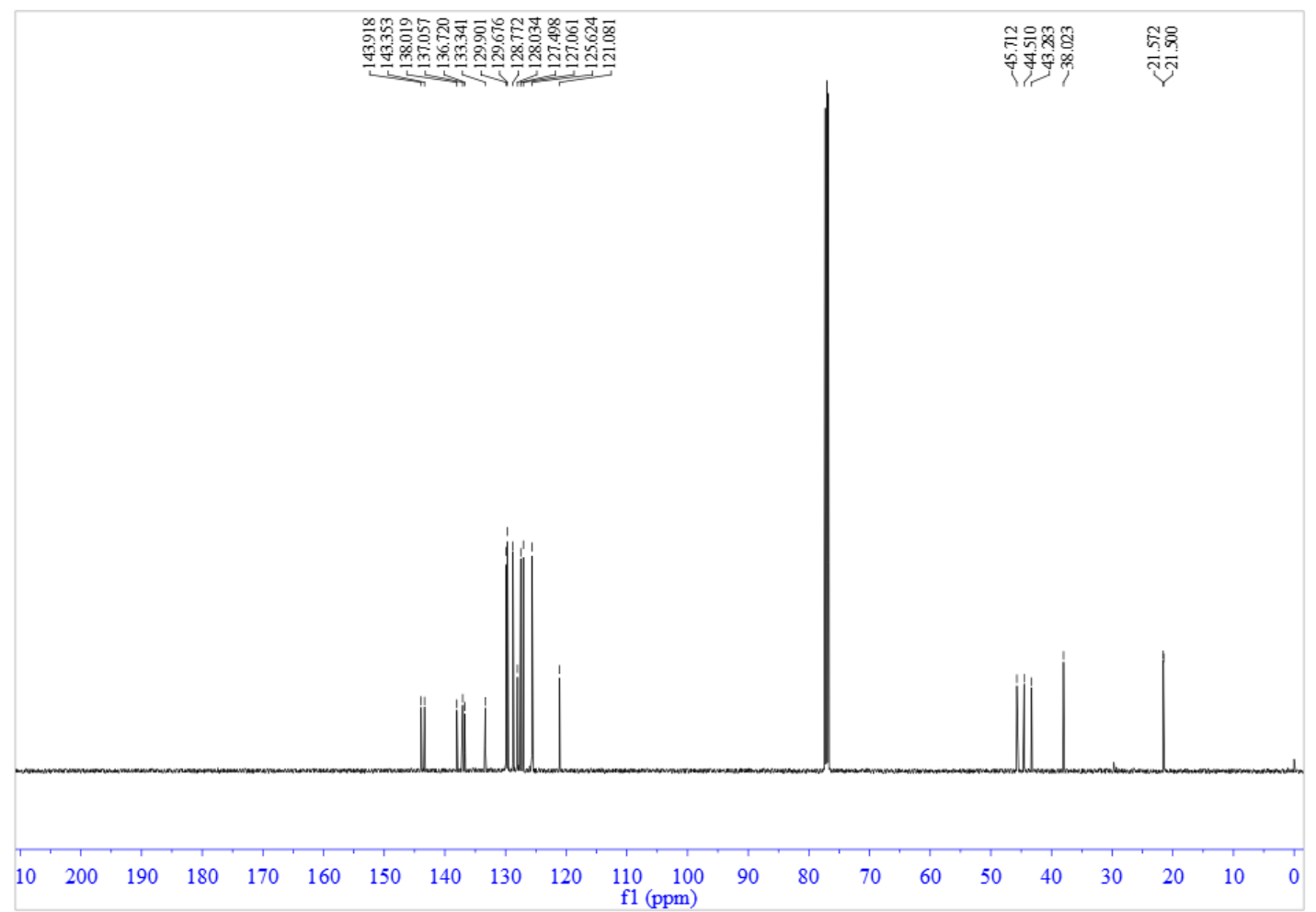




\section{${ }^{13} \mathrm{C}$ APT of compound $\mathbf{6 a}$}

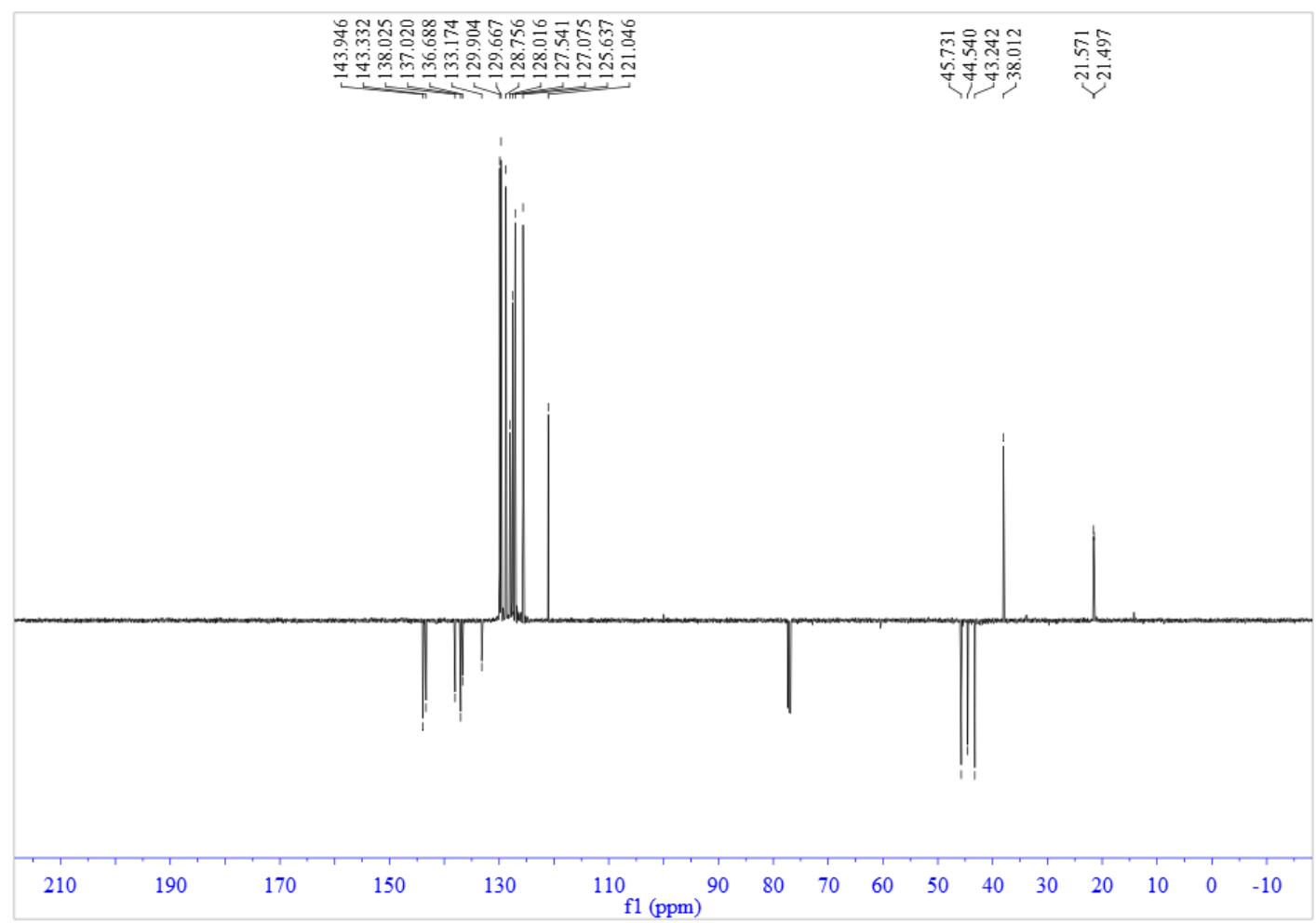


${ }^{1} \mathrm{H}$ NMR $\left(500 \mathrm{MHz}, \mathrm{CDCl}_{3}\right)$ of compound $\mathbf{6 b}$

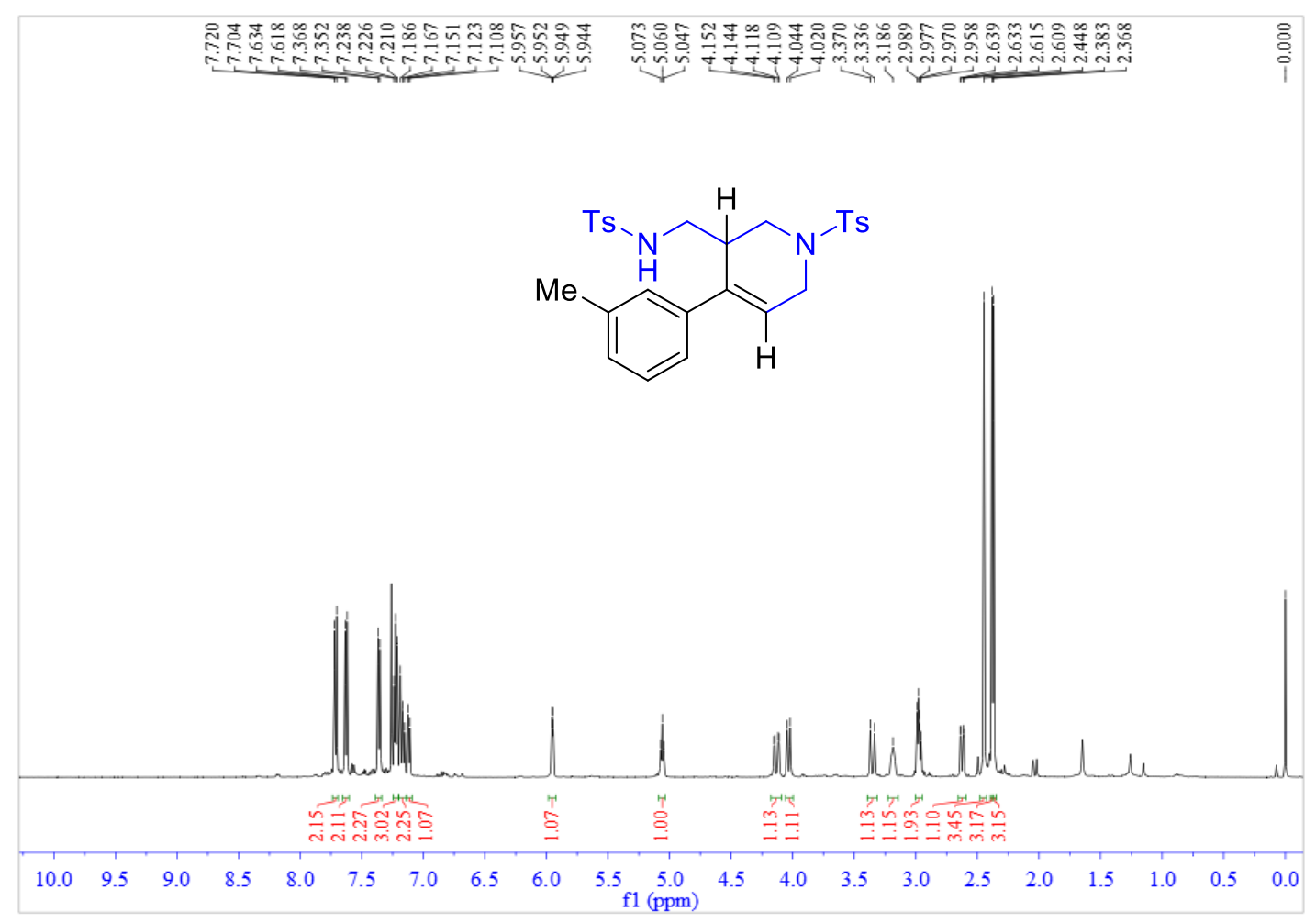

${ }^{13} \mathrm{C}$ NMR $\left(125 \mathrm{MHz}, \mathrm{CDCl}_{3}\right.$ ) of compound $\mathbf{6 b}$

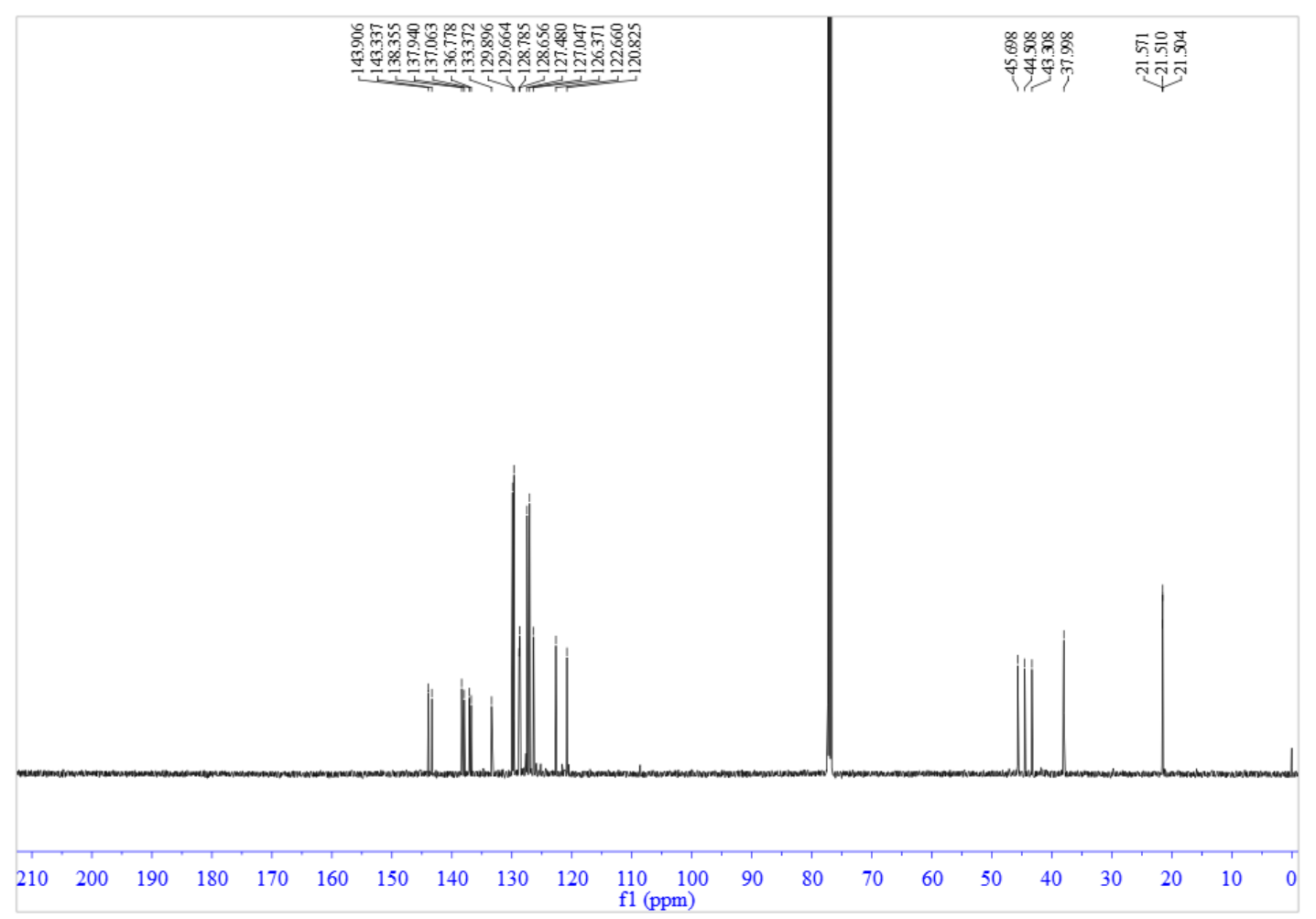


${ }^{1} \mathrm{H}$ NMR $\left(500 \mathrm{MHz}, \mathrm{CDCl}_{3}\right.$ ) of compound $\mathbf{6 c}$

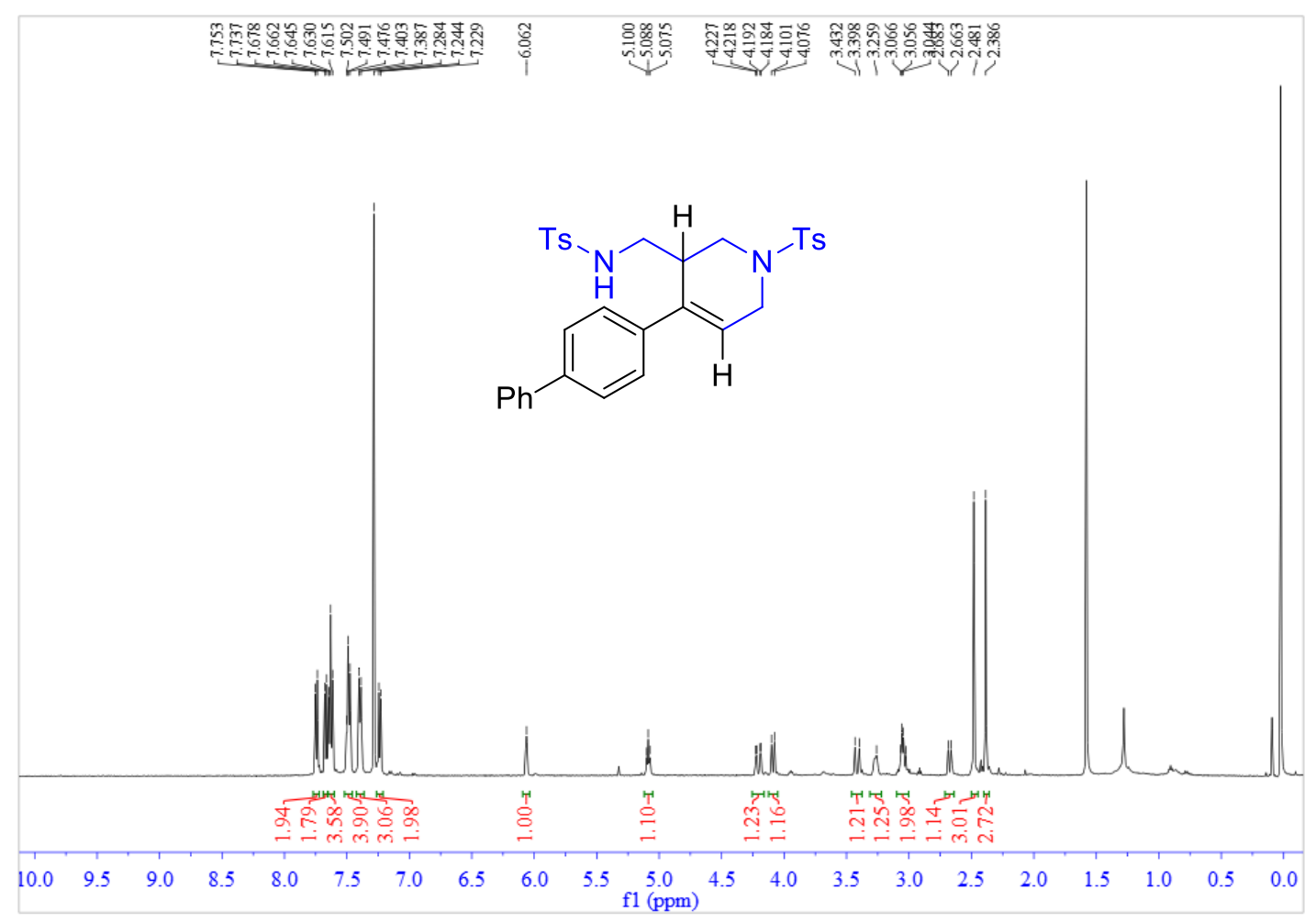

${ }^{13} \mathrm{C}$ NMR (125 MHz, $\mathrm{CDCl}_{3}$ ) of compound $\mathbf{6 c}$

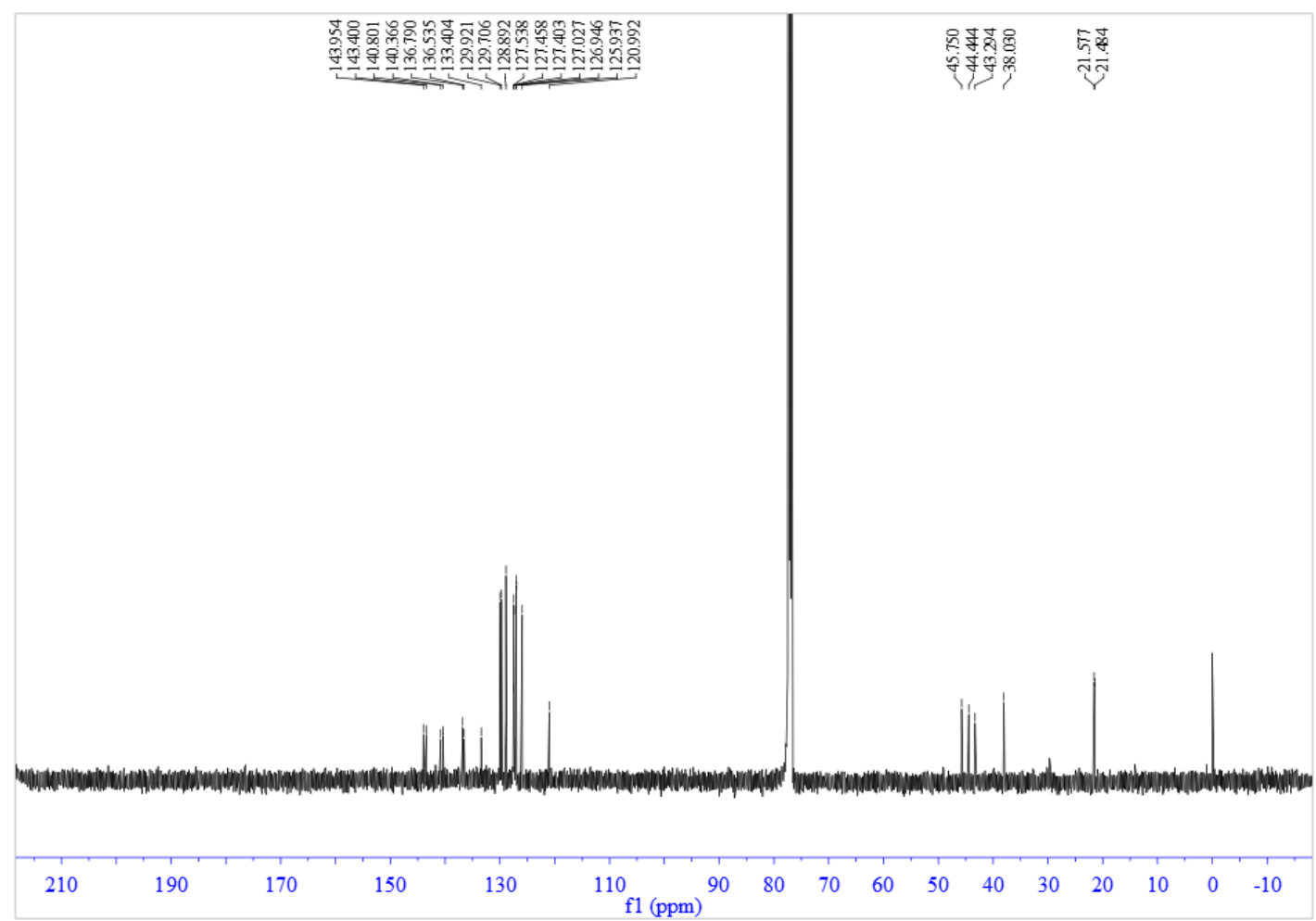


${ }^{1} \mathrm{H}$ NMR $\left(500 \mathrm{MHz}, \mathrm{CDCl}_{3}\right)$ of compound $\mathbf{6 d}$

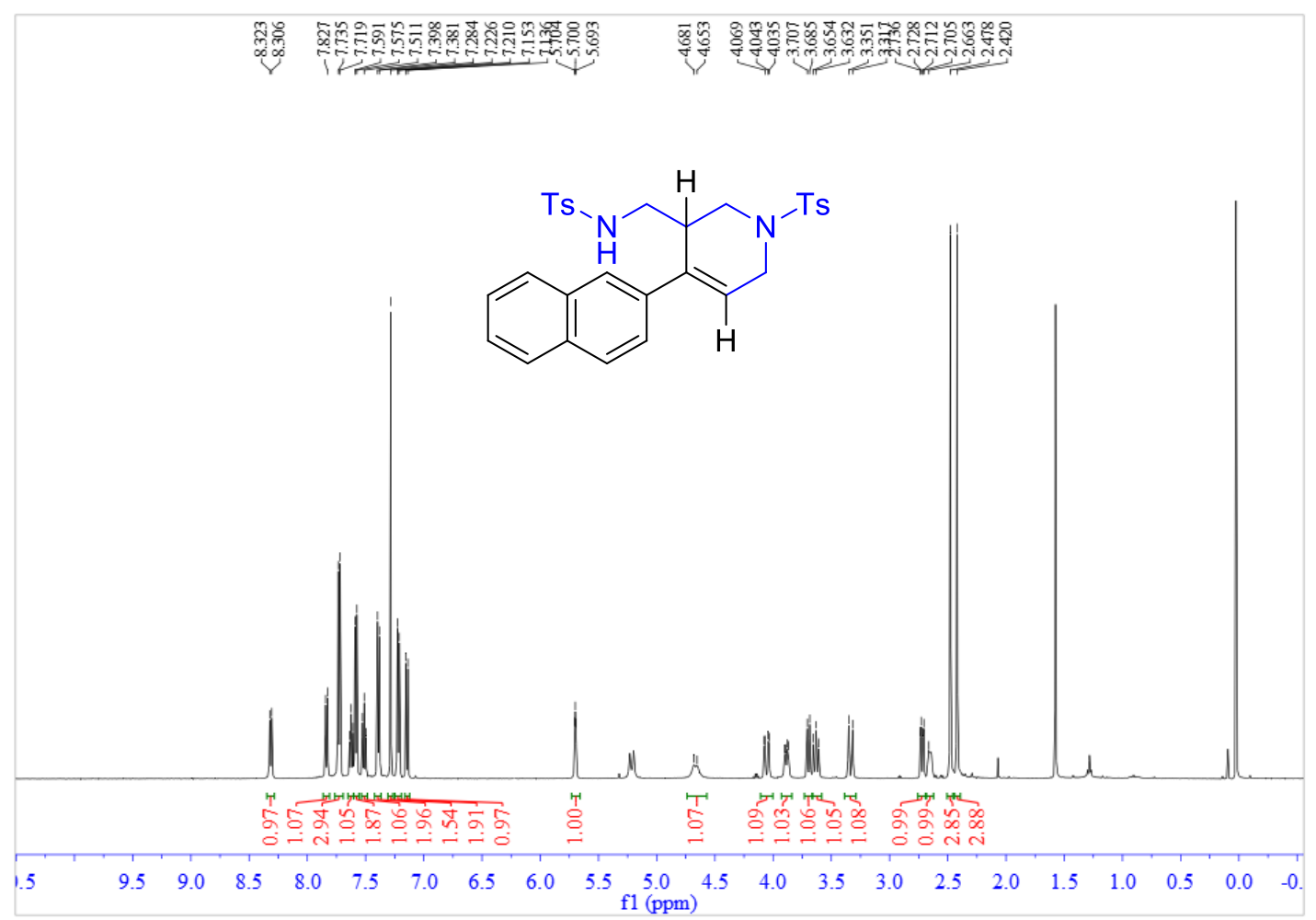

${ }^{13} \mathrm{C}$ NMR $\left(125 \mathrm{MHz}, \mathrm{CDCl}_{3}\right)$ of compound $\mathbf{6 d}$

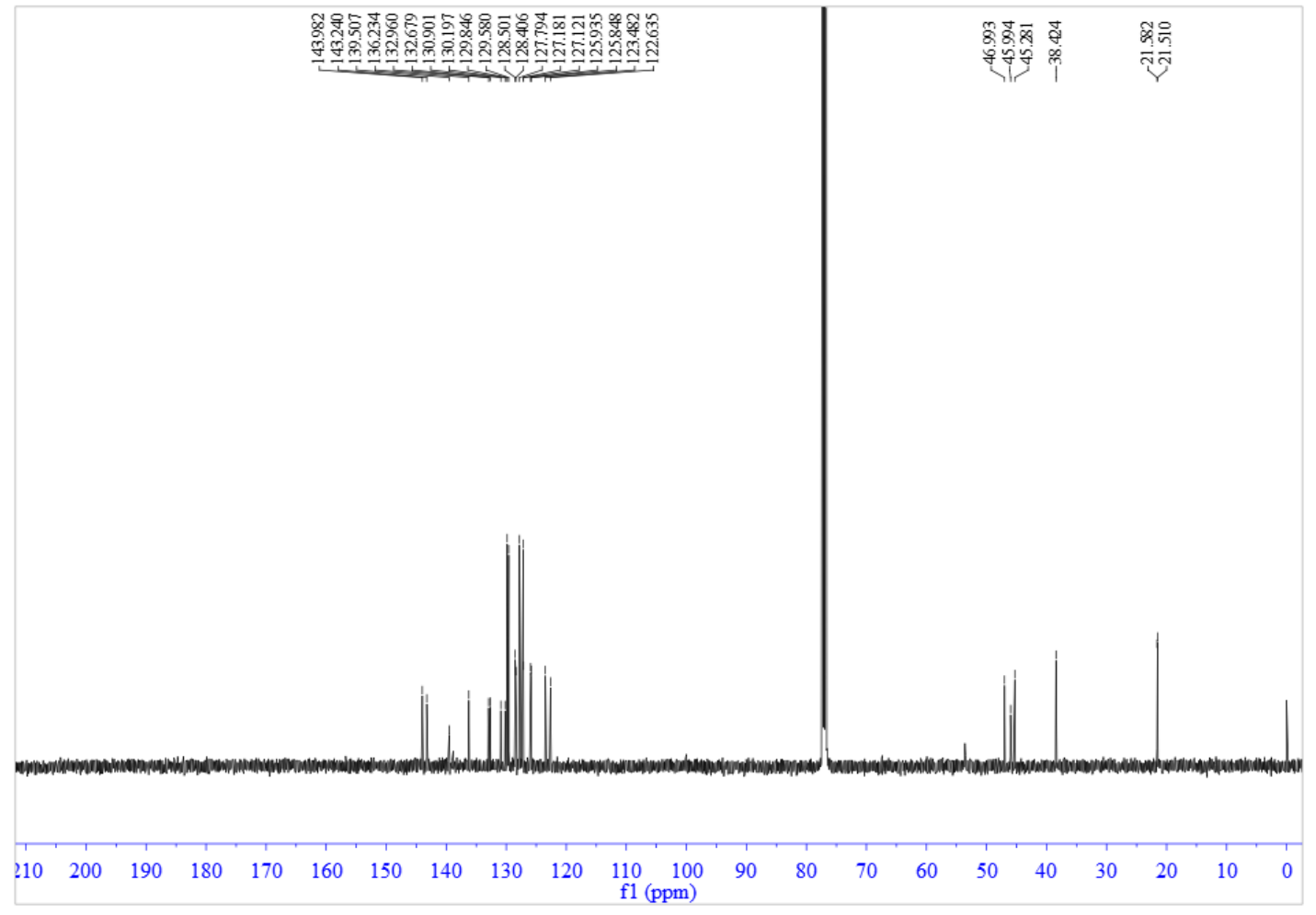


${ }^{1} \mathrm{H}$ NMR $\left(500 \mathrm{MHz}, \mathrm{CDCl}_{3}\right)$ of compound $\mathbf{6 e}$

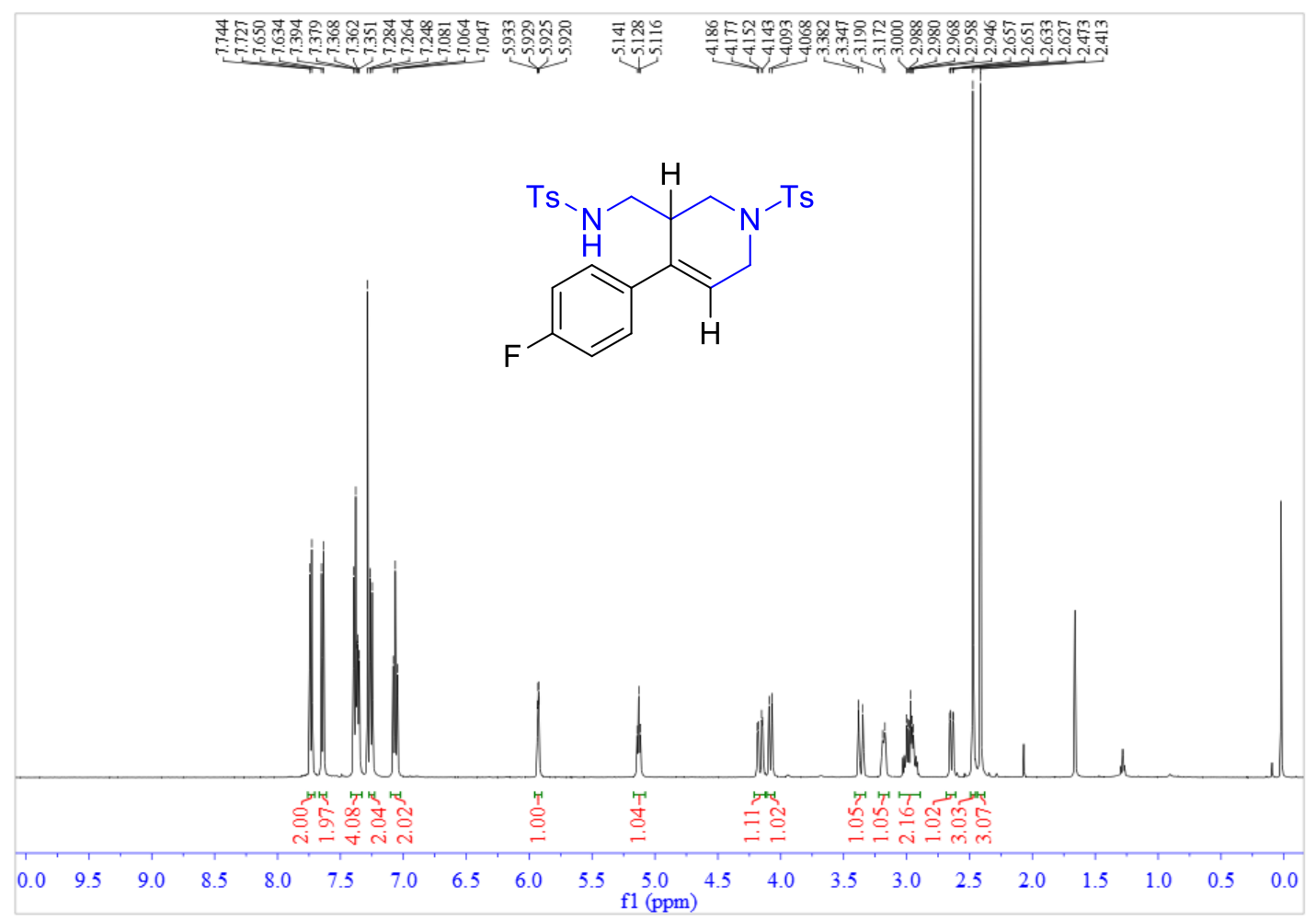

${ }^{13} \mathrm{C}$ NMR (125 MHz, $\left.\mathrm{CDCl}_{3}\right)$ of compound $\mathbf{6 e}$

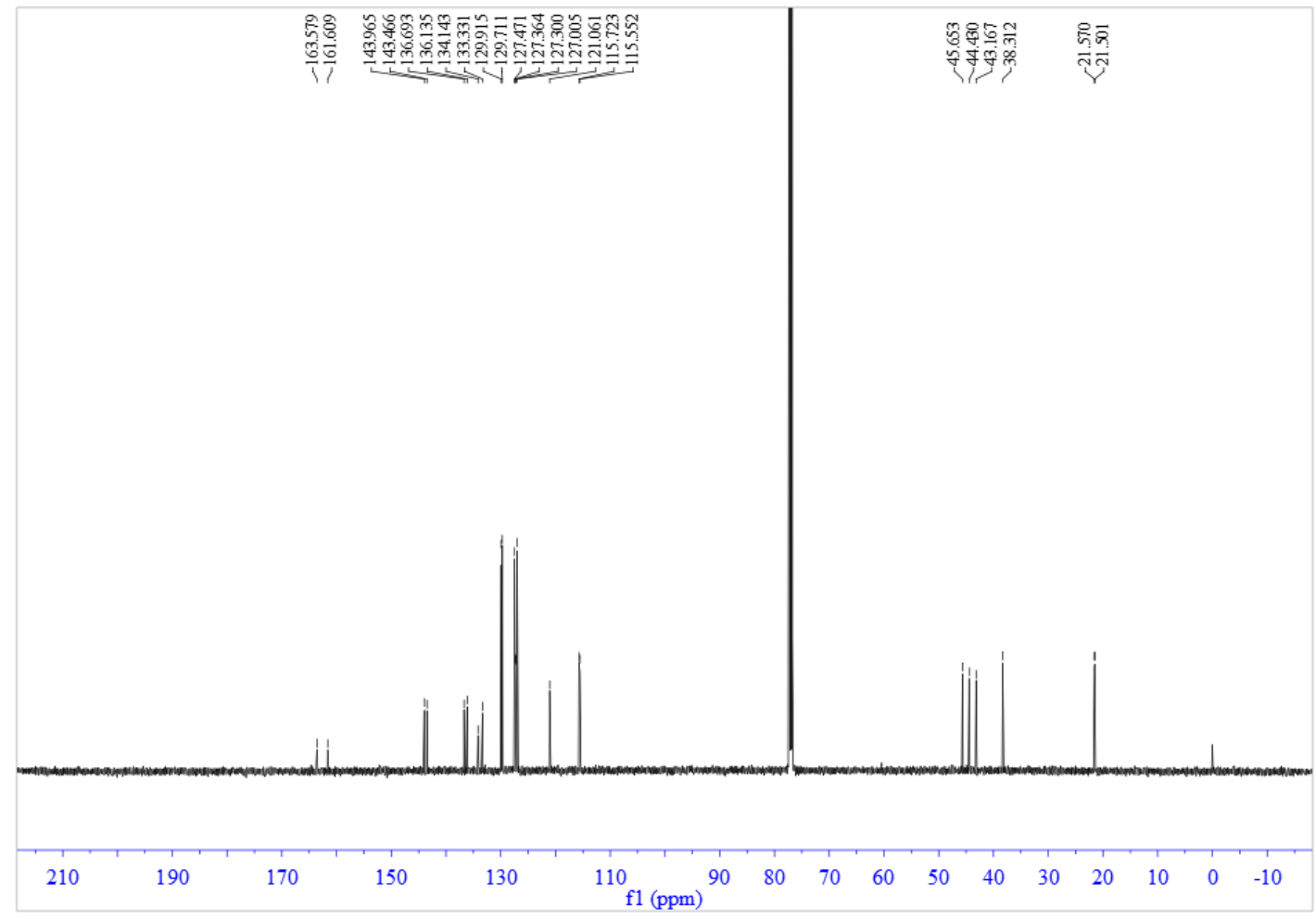


${ }^{19} \mathrm{~F}$ NMR (470 MHz, $\mathrm{CDCl}_{3}$ ) of compound $\mathbf{6 e}$

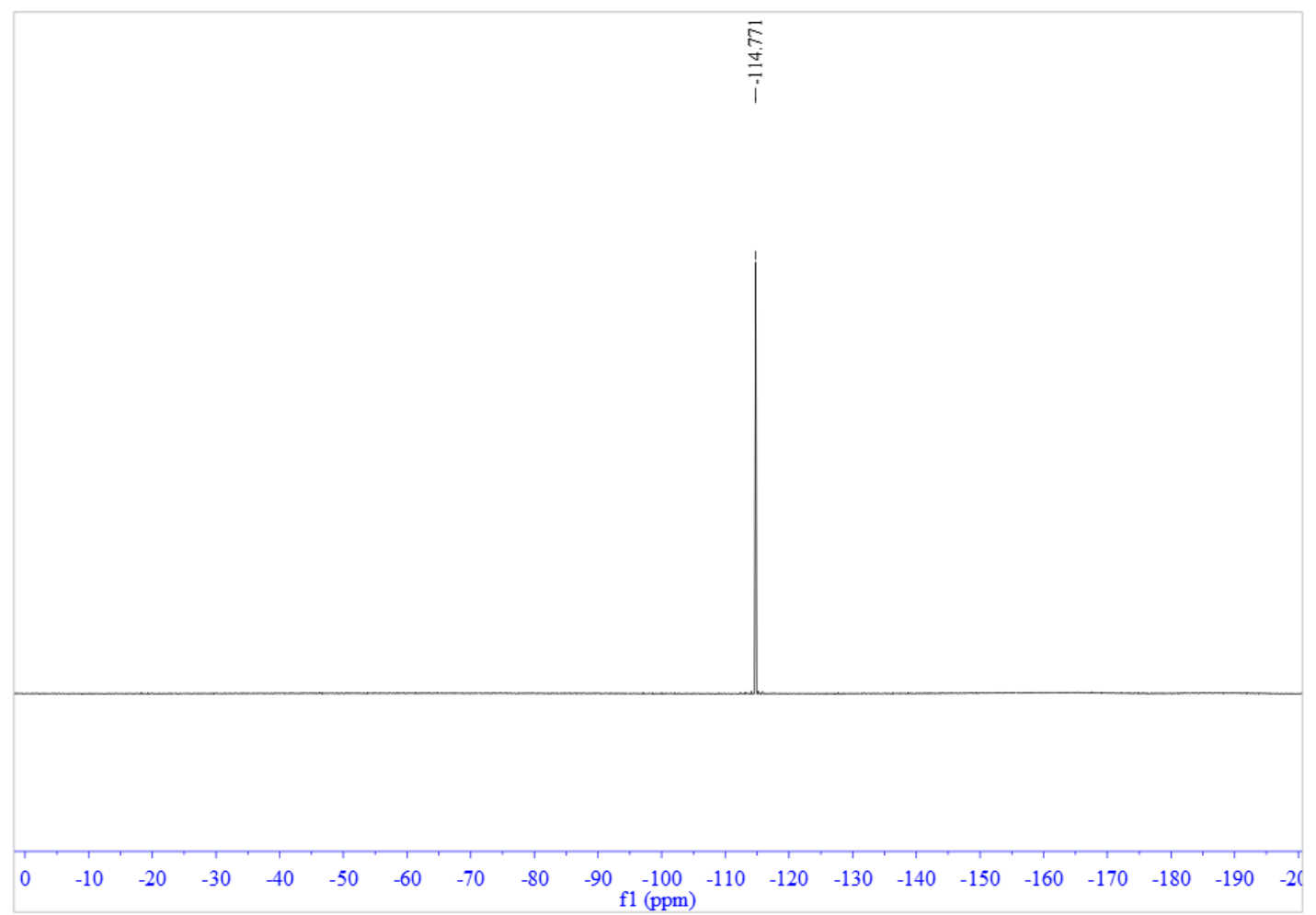


${ }^{1} \mathrm{H}$ NMR $\left(500 \mathrm{MHz}, \mathrm{CDCl}_{3}\right)$ of compound $\mathbf{6 f}$

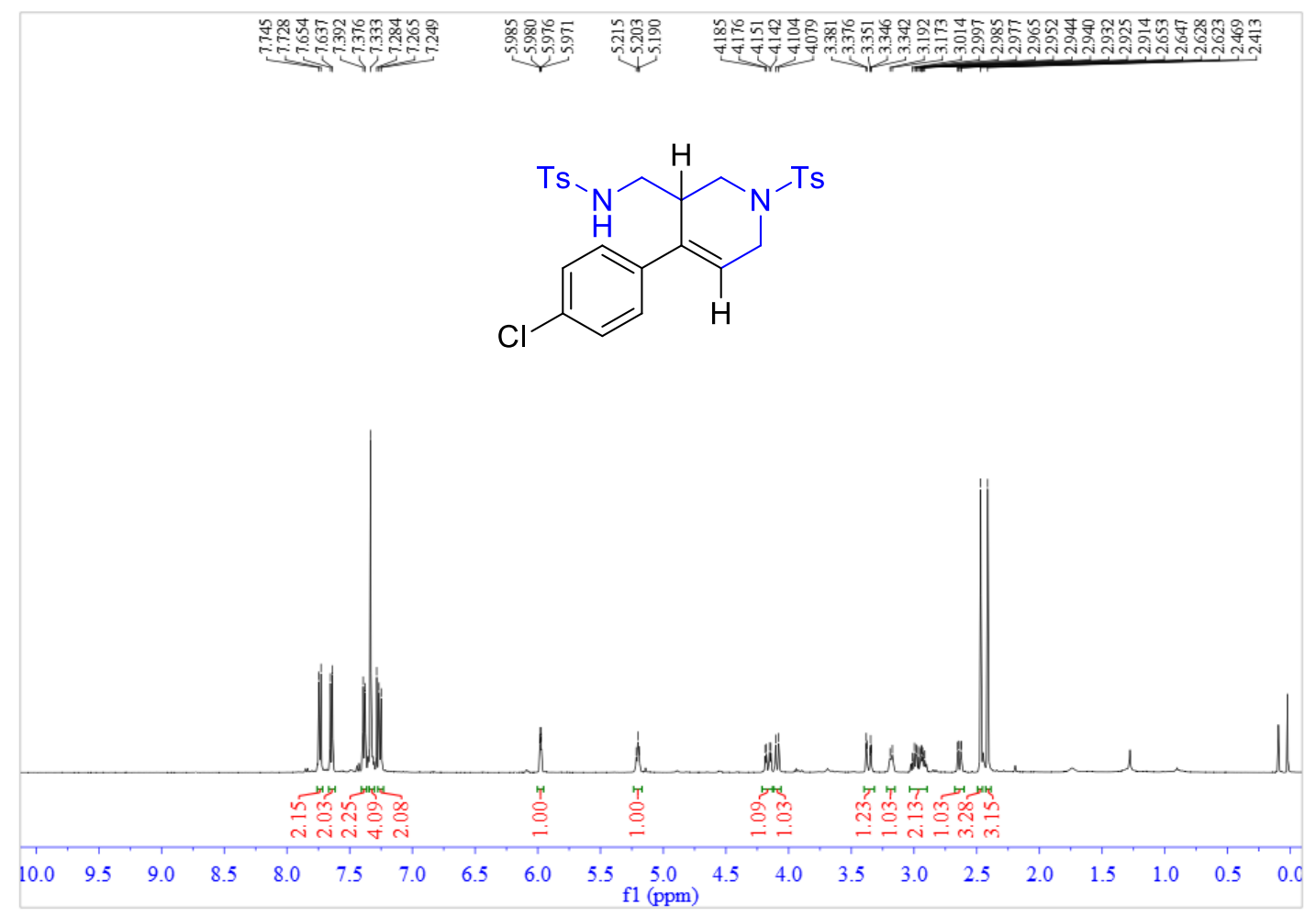

${ }^{13} \mathrm{C}$ NMR (125 MHz, $\left.\mathrm{CDCl}_{3}\right)$ of compound $\mathbf{6 f}$

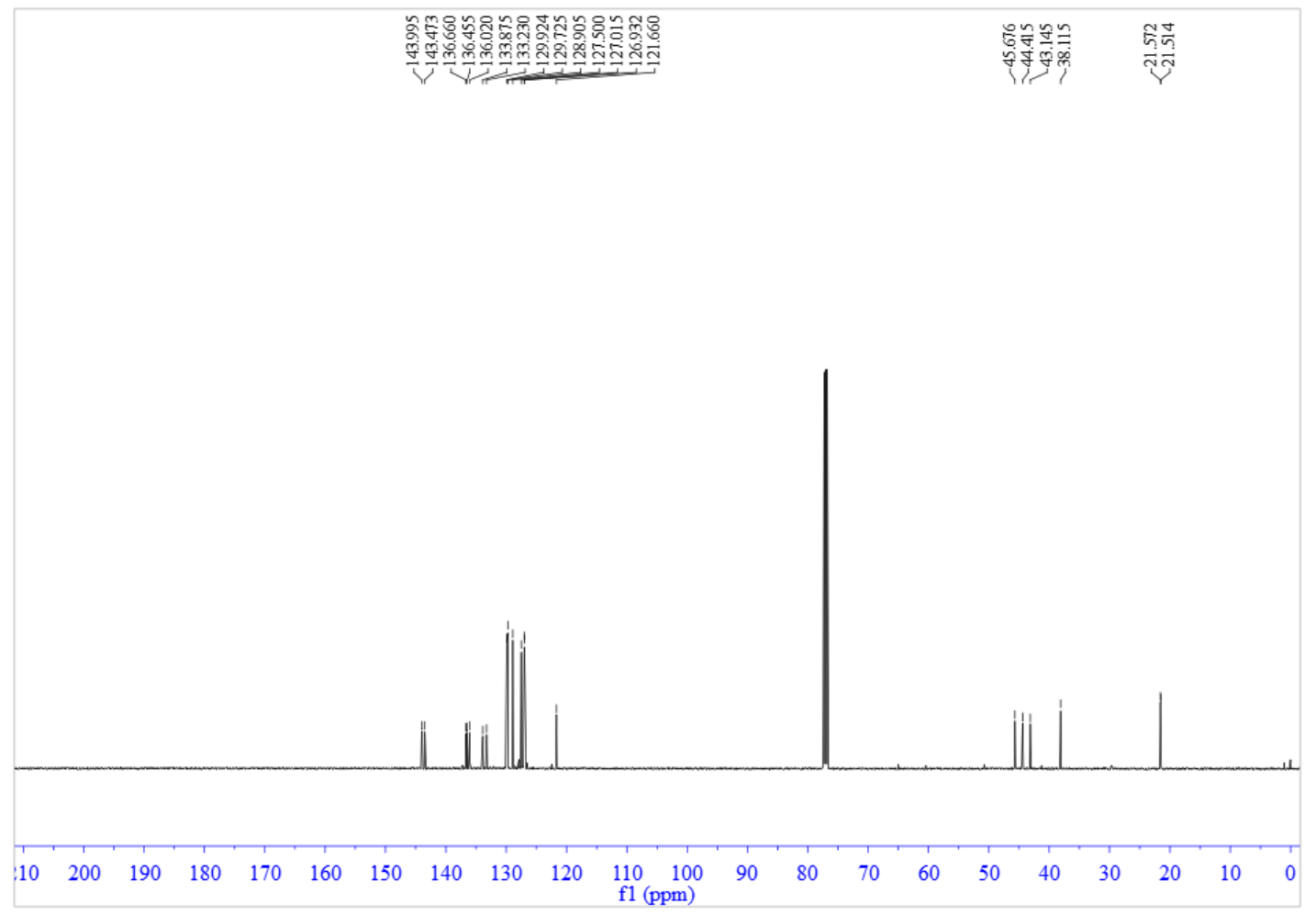


${ }^{1} \mathrm{H}$ NMR $\left(500 \mathrm{MHz}, \mathrm{CDCl}_{3}\right)$ of compound $\mathbf{6 g}$

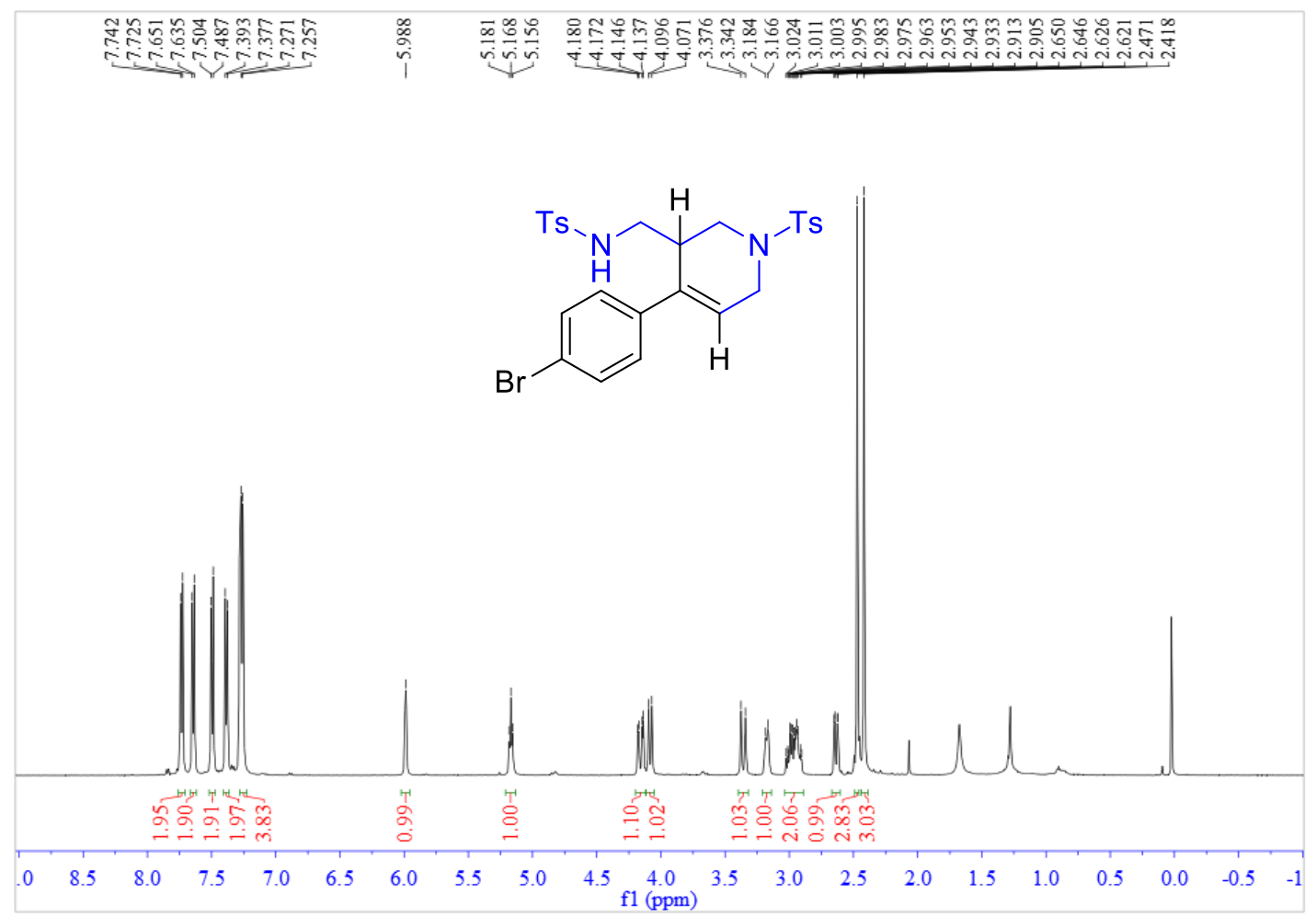

${ }^{13} \mathrm{C}$ NMR (125 MHz, $\mathrm{CDCl}_{3}$ ) of compound $\mathbf{6 g}$

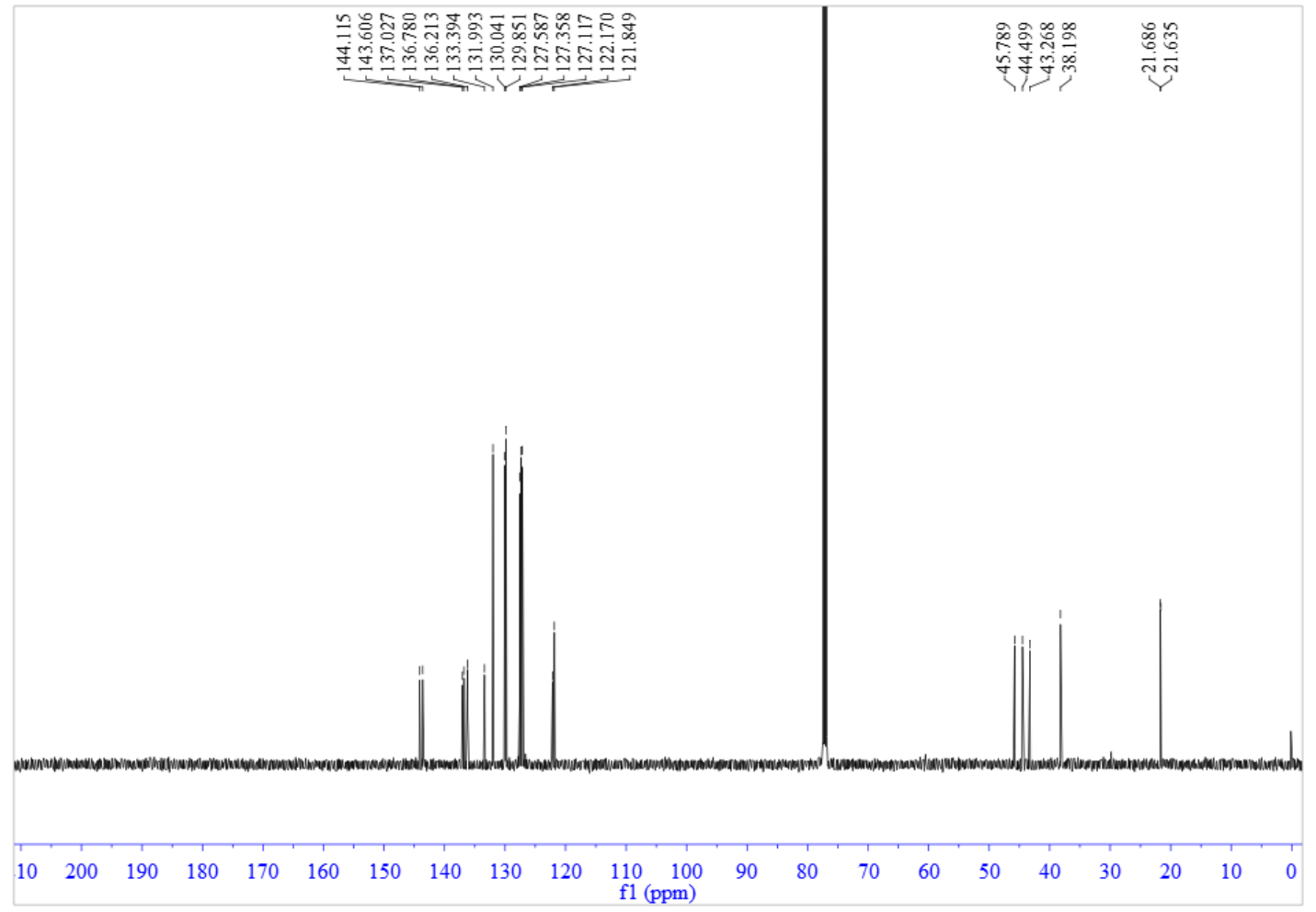


${ }^{1} \mathrm{H}$ NMR $\left(500 \mathrm{MHz}, \mathrm{CDCl}_{3}\right)$ of compound $\mathbf{6} \mathbf{h}$

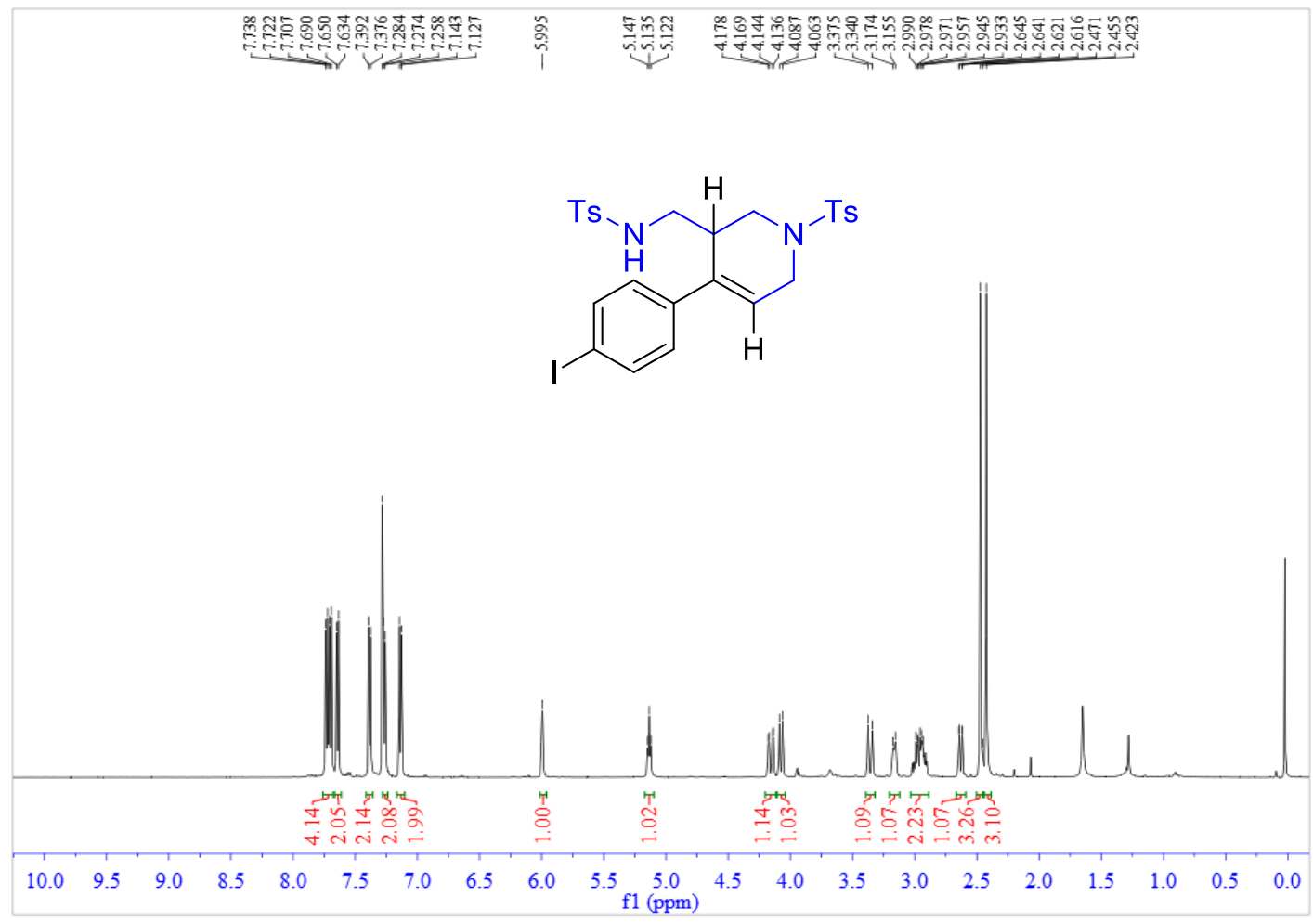

${ }^{13} \mathrm{C}$ NMR $\left(125 \mathrm{MHz}, \mathrm{CDCl}_{3}\right)$ of compound $\mathbf{6 h}$

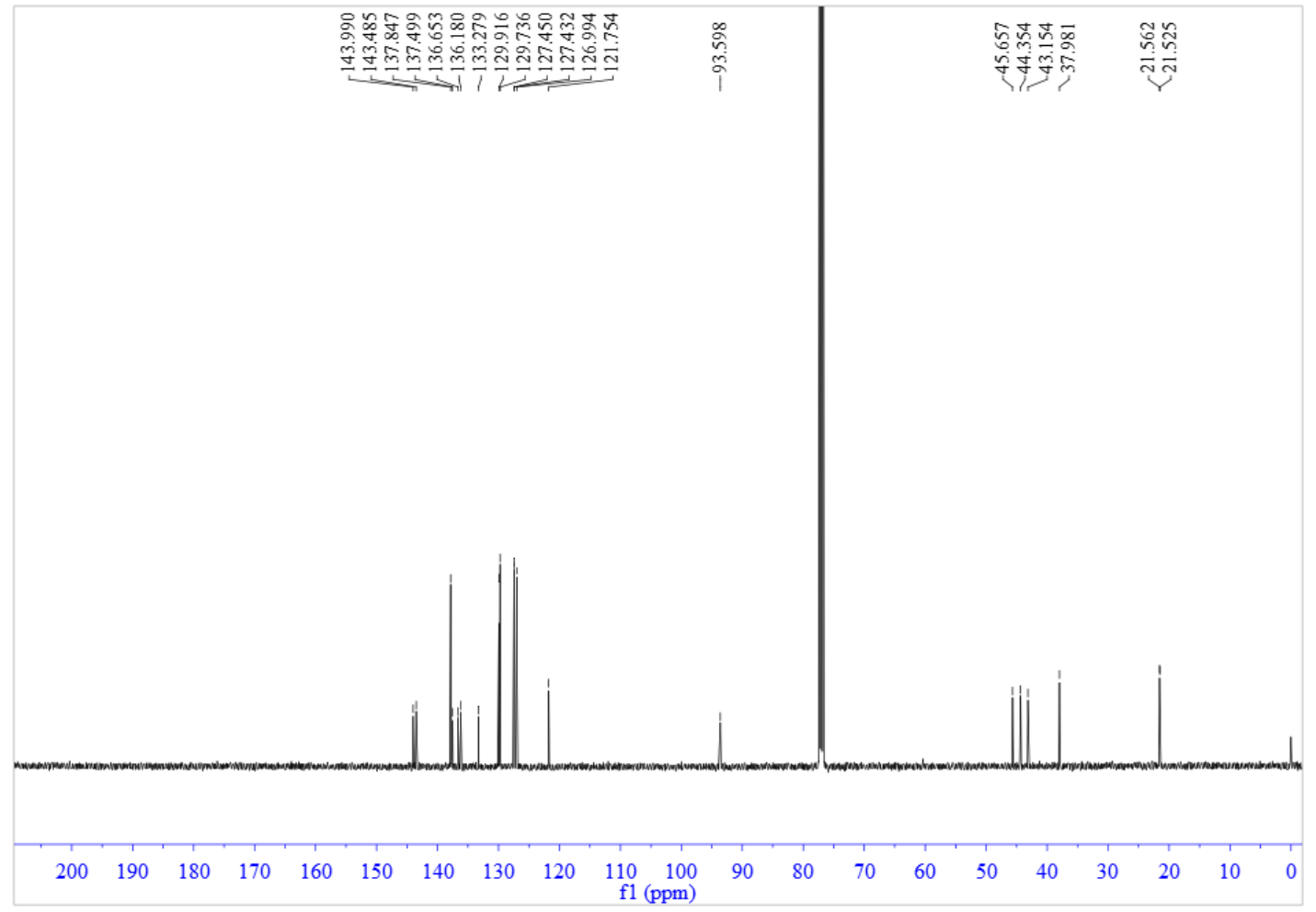


${ }^{1} \mathrm{H}$ NMR $\left(500 \mathrm{MHz}, \mathrm{CDCl}_{3}\right.$ ) of compound $\mathbf{6 i}$

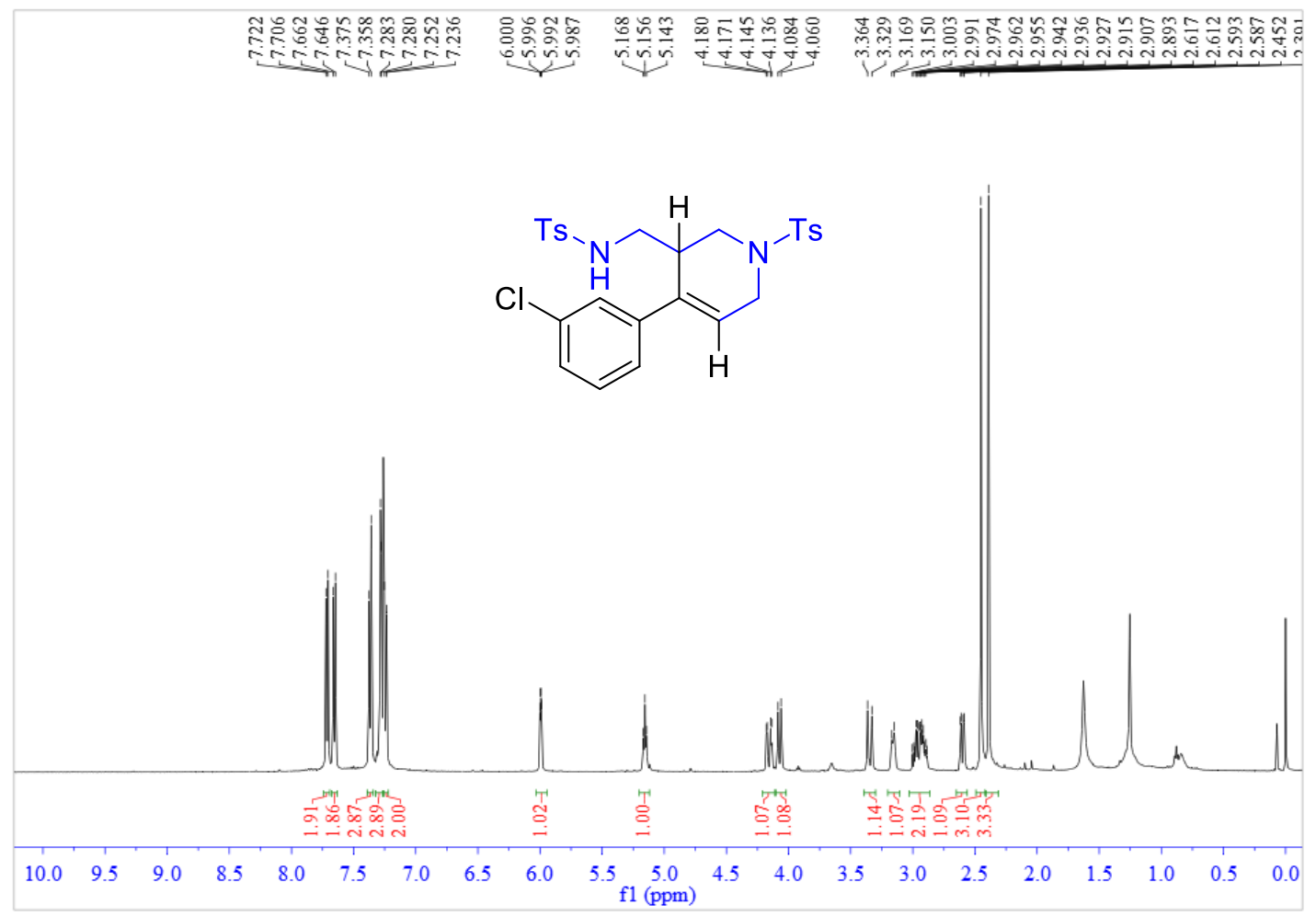

${ }^{13} \mathrm{C}$ NMR (125 MHz, $\left.\mathrm{CDCl}_{3}\right)$ of compound $\mathbf{6 i}$

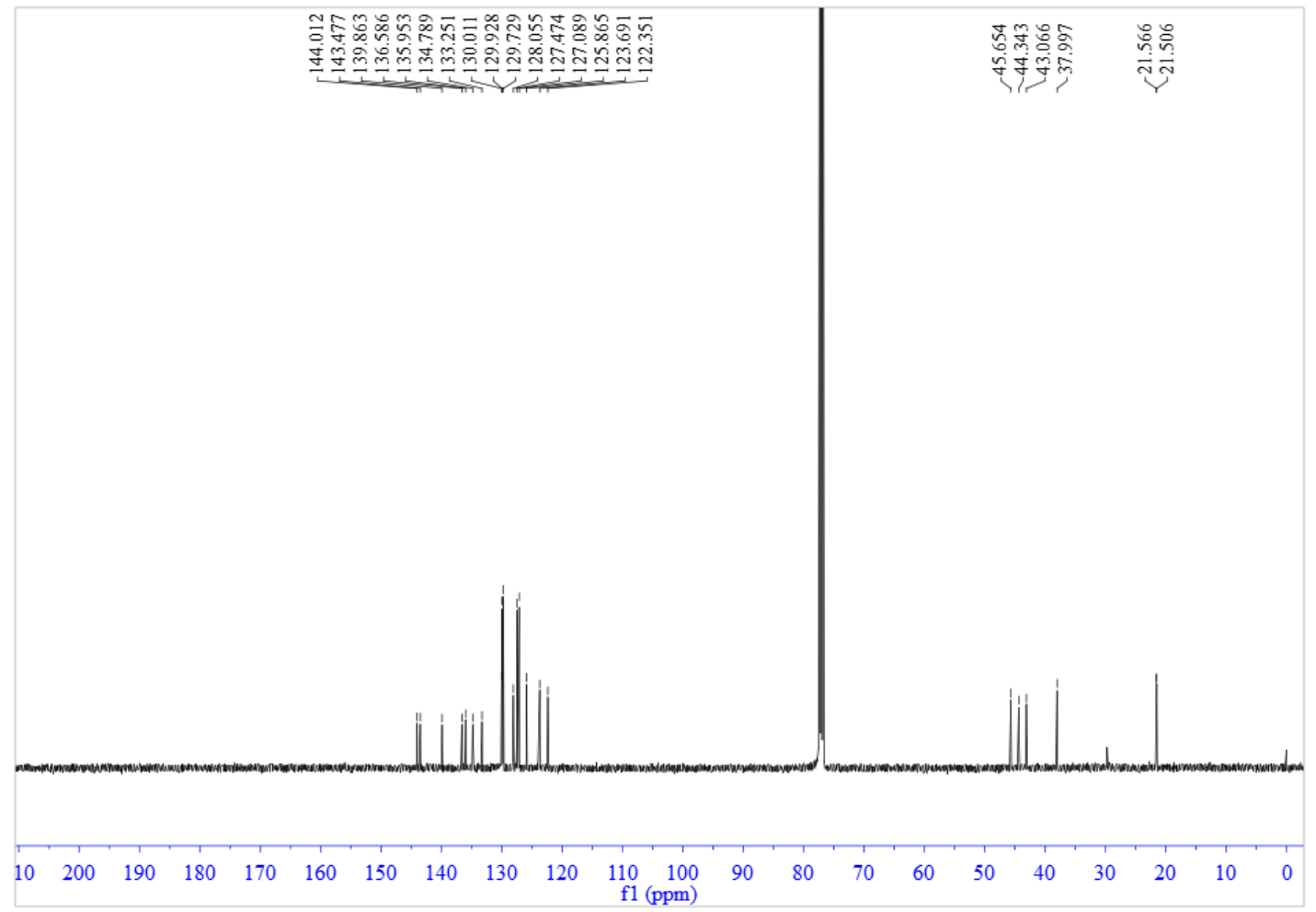


${ }^{1} \mathrm{H}$ NMR $\left(500 \mathrm{MHz}, \mathrm{CDCl}_{3}\right)$ of compound $\mathbf{6 j}$

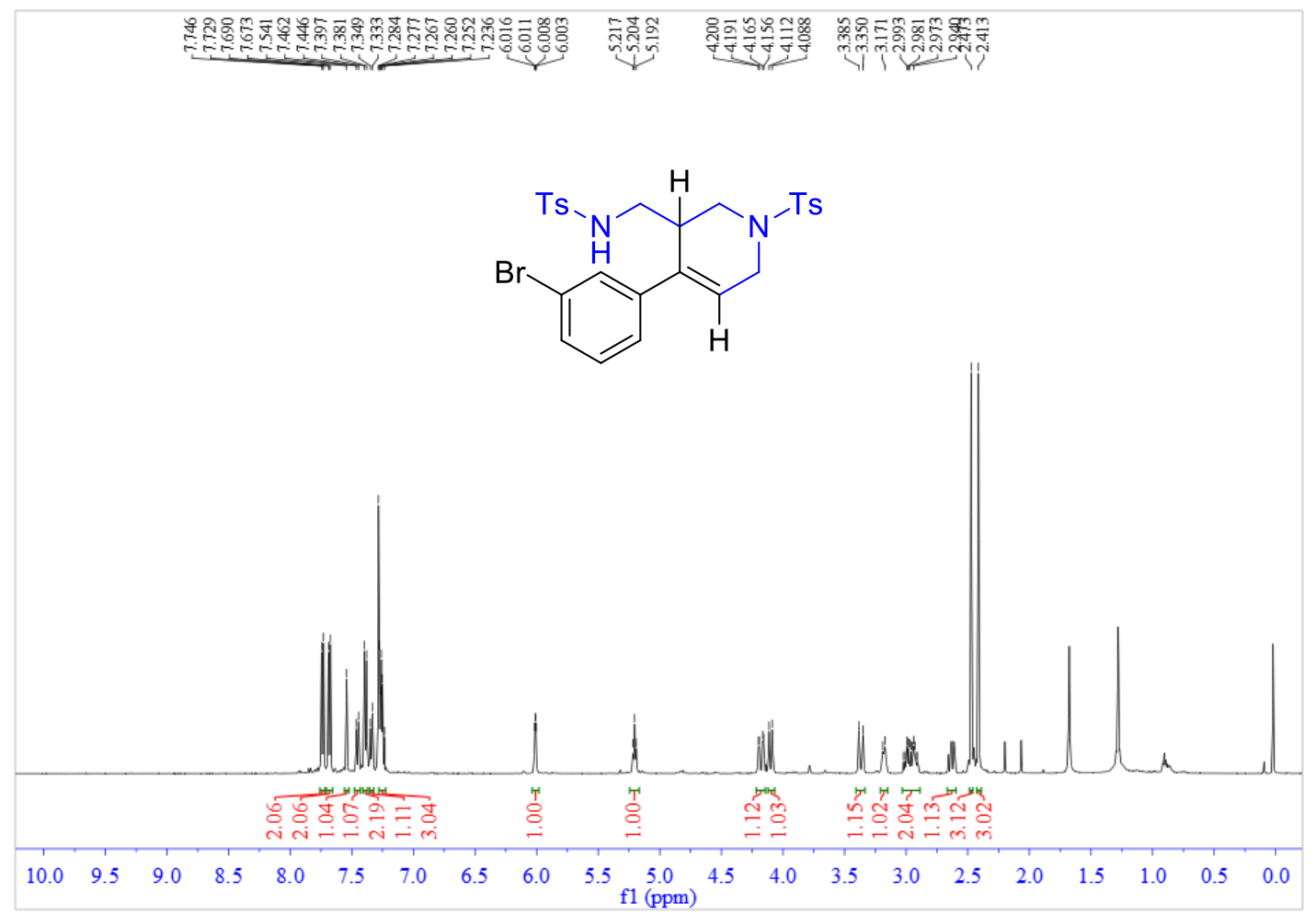

${ }^{13} \mathrm{C}$ NMR (125 MHz, $\mathrm{CDCl}_{3}$ ) of compound $\mathbf{6 j}$

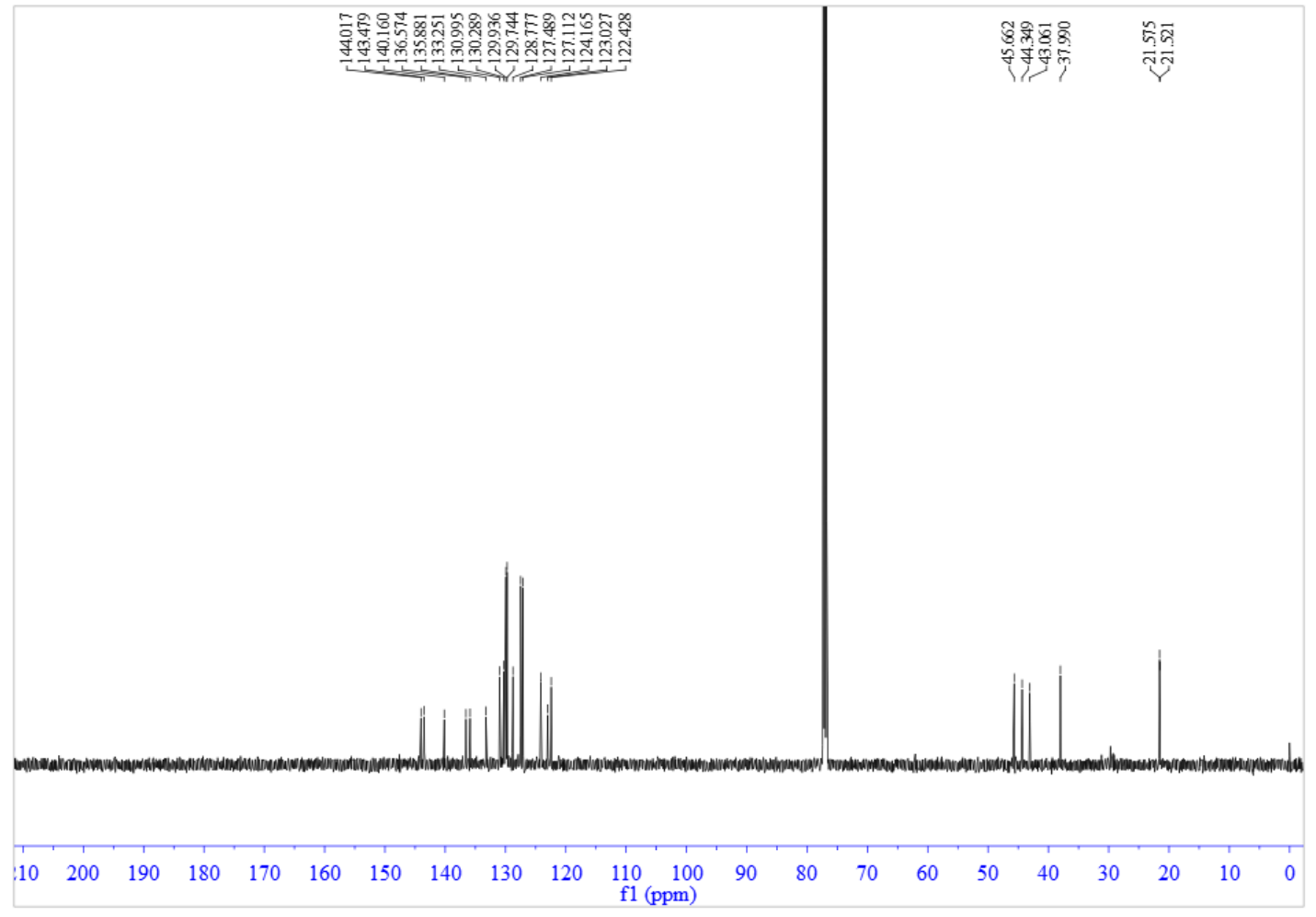


${ }^{1} \mathrm{H}$ NMR $\left(500 \mathrm{MHz}, \mathrm{CDCl}_{3}\right)$ of compound $\mathbf{6 k}$

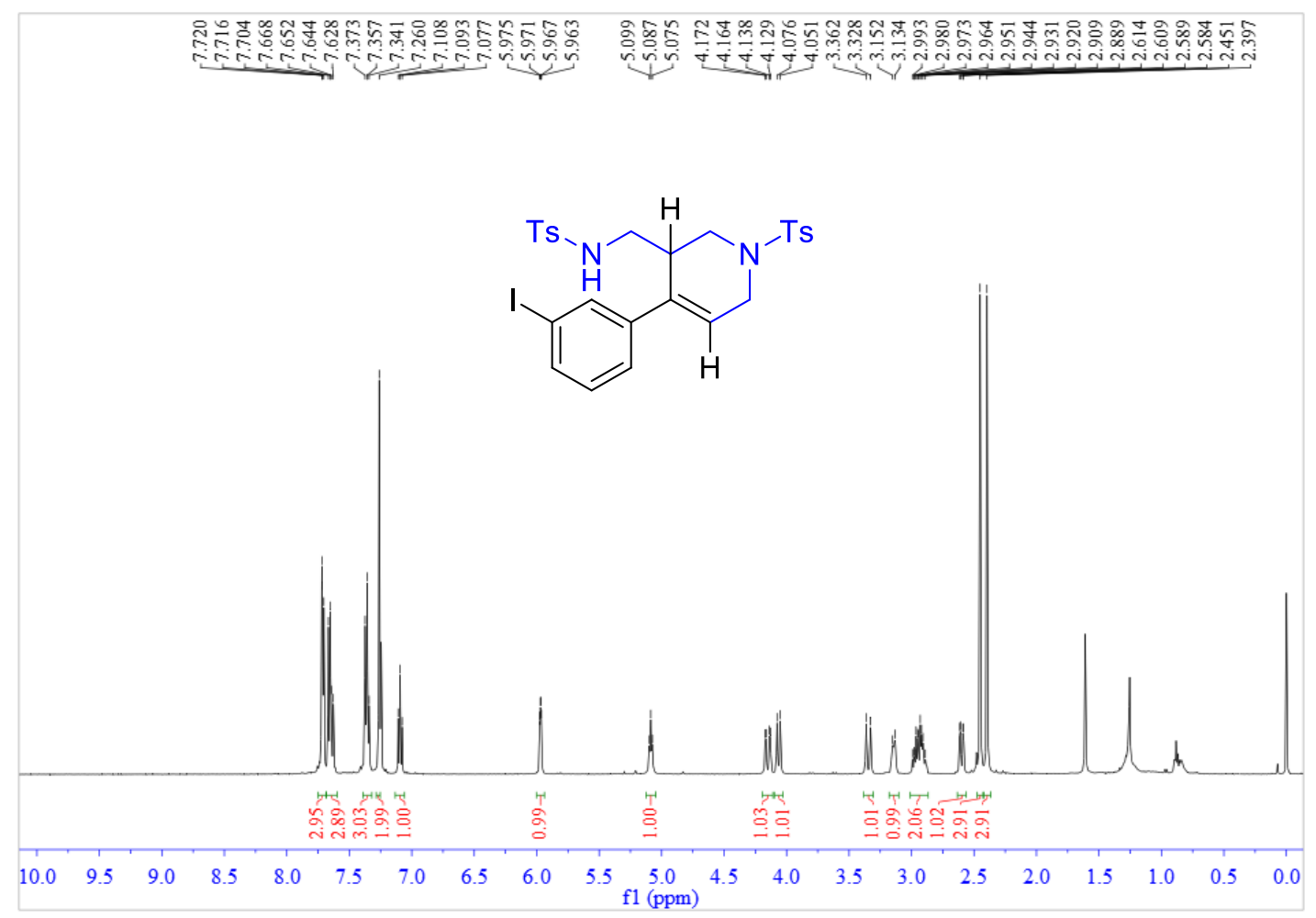

${ }^{13} \mathrm{C}$ NMR (125 MHz, $\mathrm{CDCl}_{3}$ ) of compound $\mathbf{6 k}$

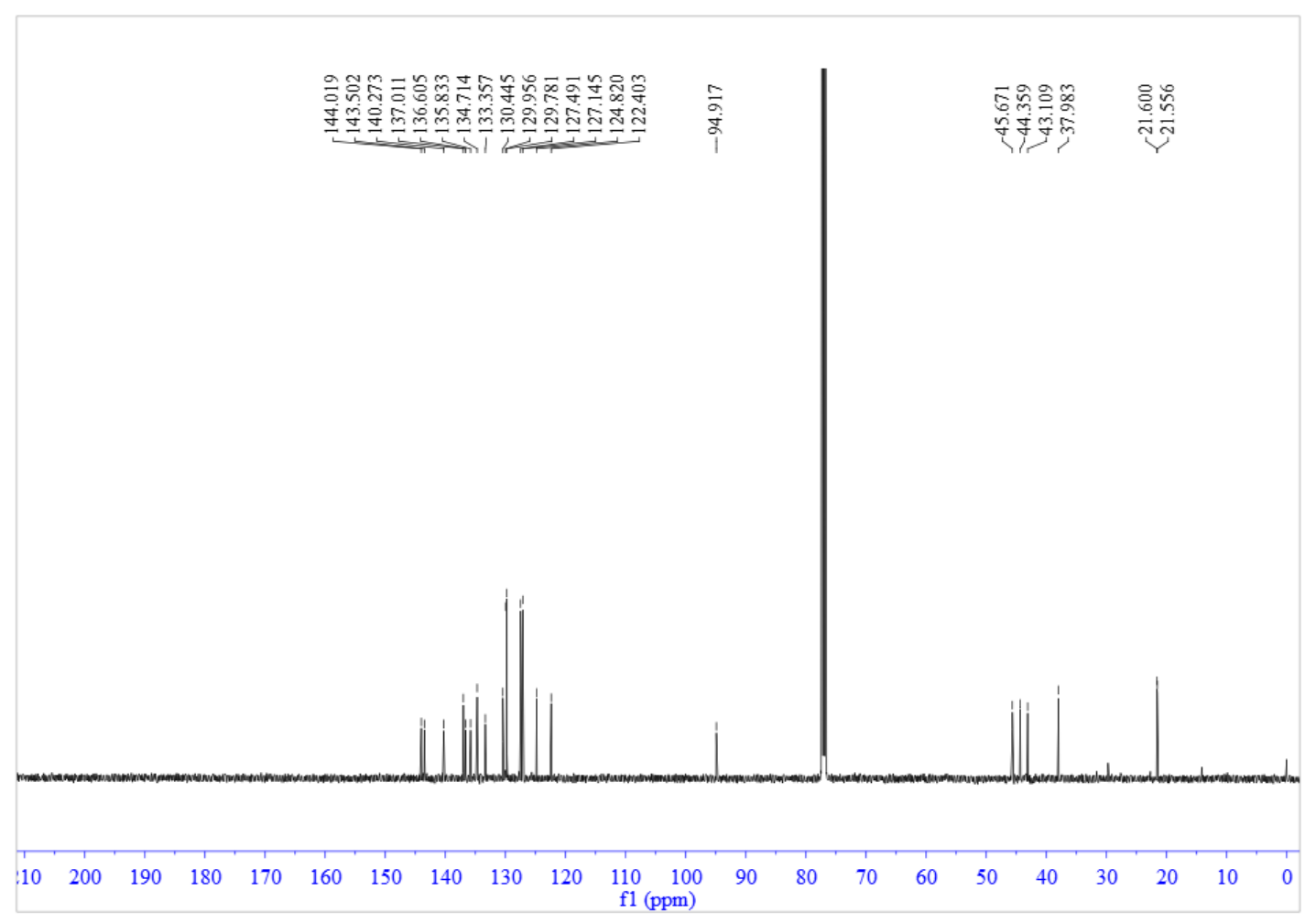


${ }^{1} \mathrm{H}$ NMR (500 MHz, $\mathrm{CDCl}_{3}$ ) of compound $6 \mathbf{l}$

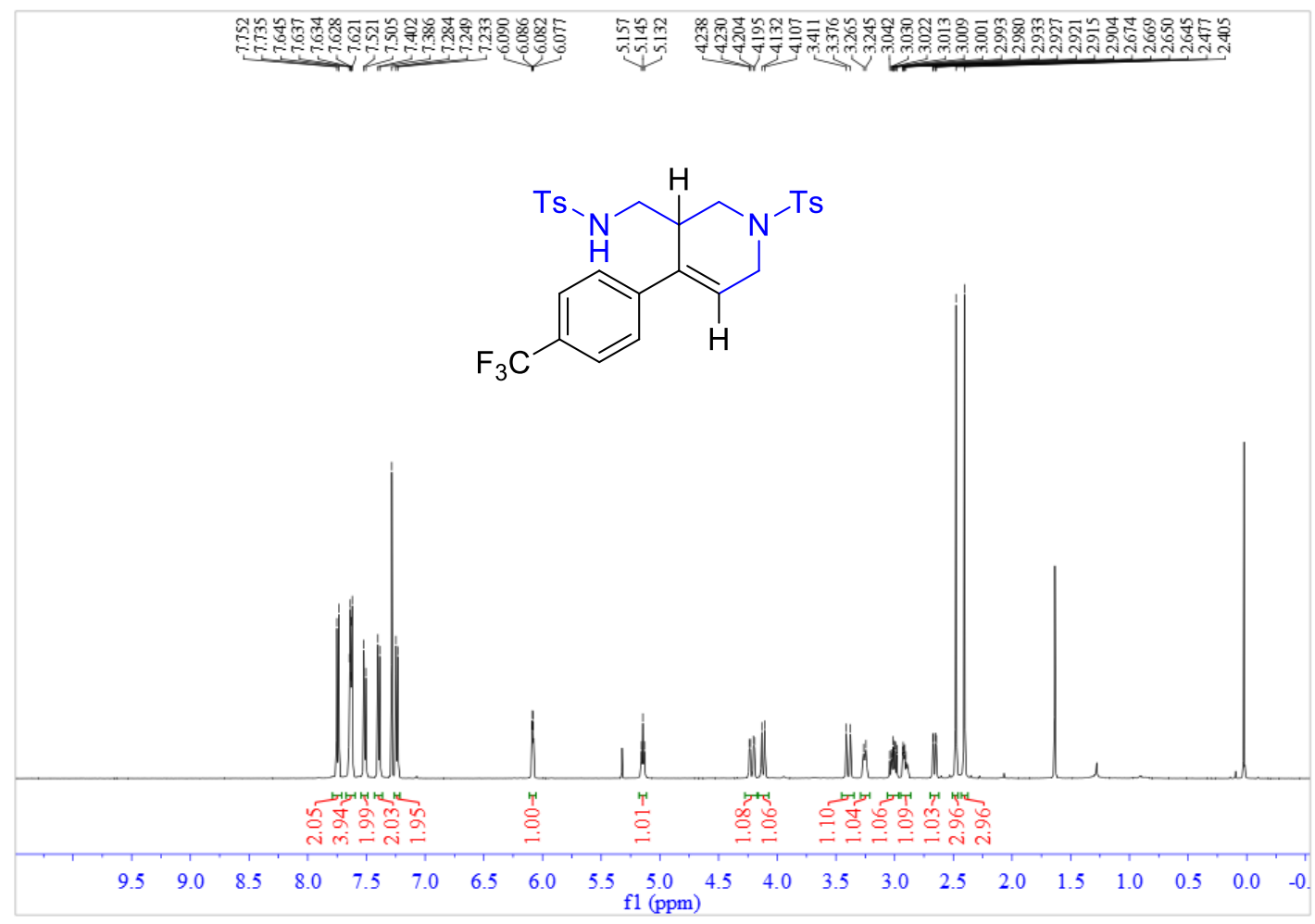

${ }^{13} \mathrm{C}$ NMR (125 MHz, $\mathrm{CDCl}_{3}$ ) of compound $6 \mathbf{l}$

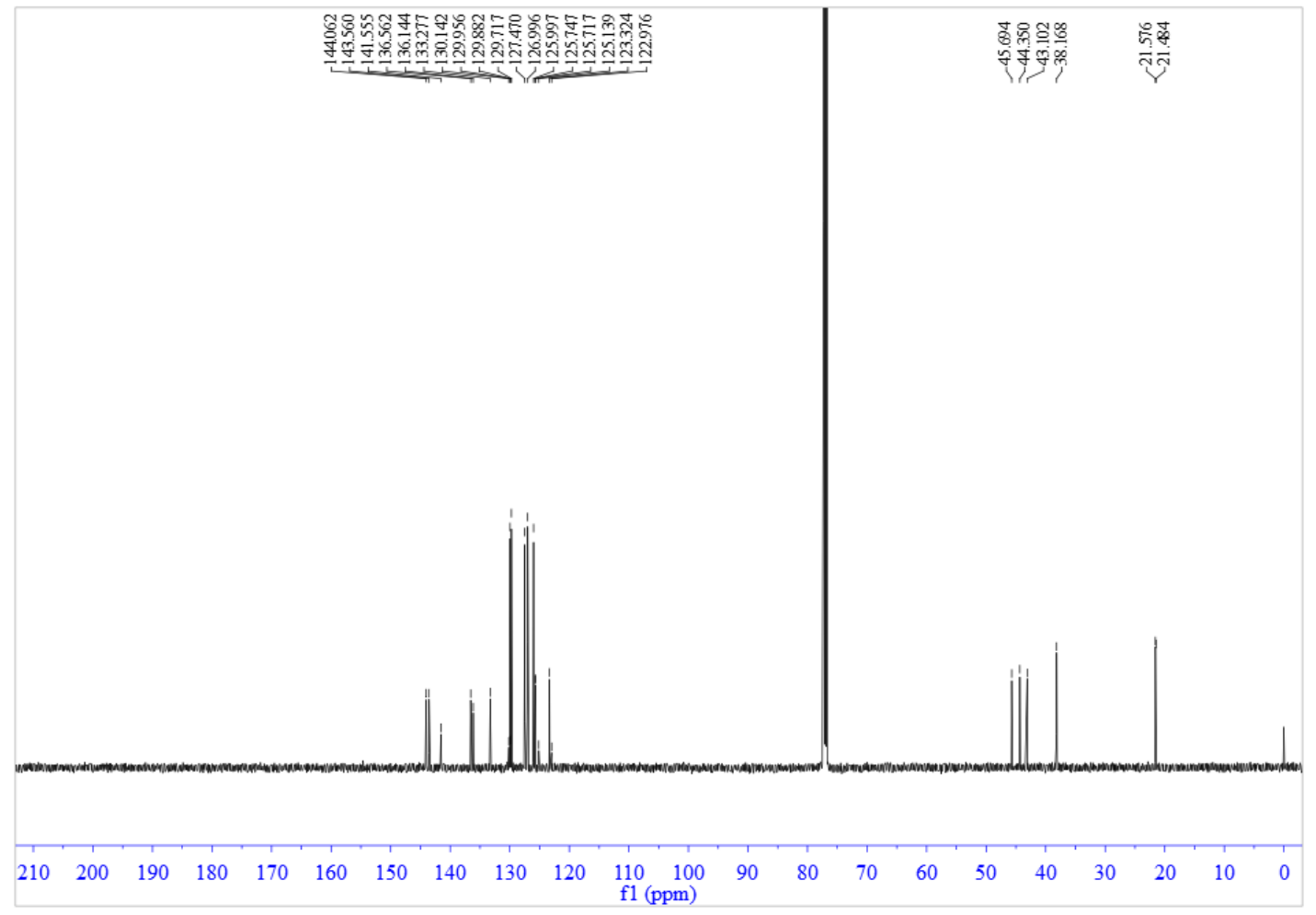


${ }^{19} \mathrm{~F}$ NMR $\left(470 \mathrm{MHz}, \mathrm{CDCl}_{3}\right)$ of compound $6 \mathbf{l}$

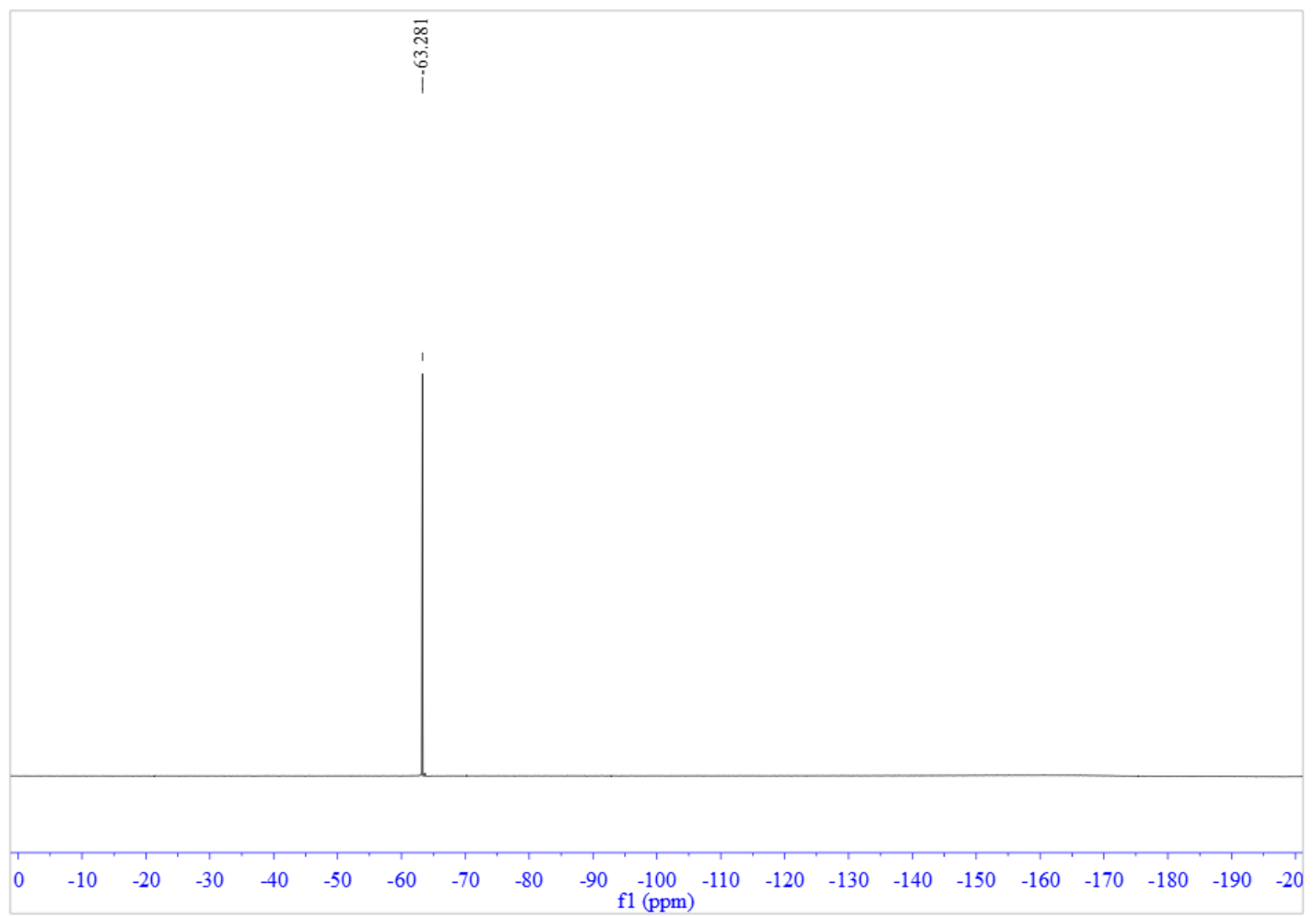


${ }^{1} \mathrm{H}$ NMR $\left(500 \mathrm{MHz}, \mathrm{CDCl}_{3}\right)$ of compound $\mathbf{6 m}$

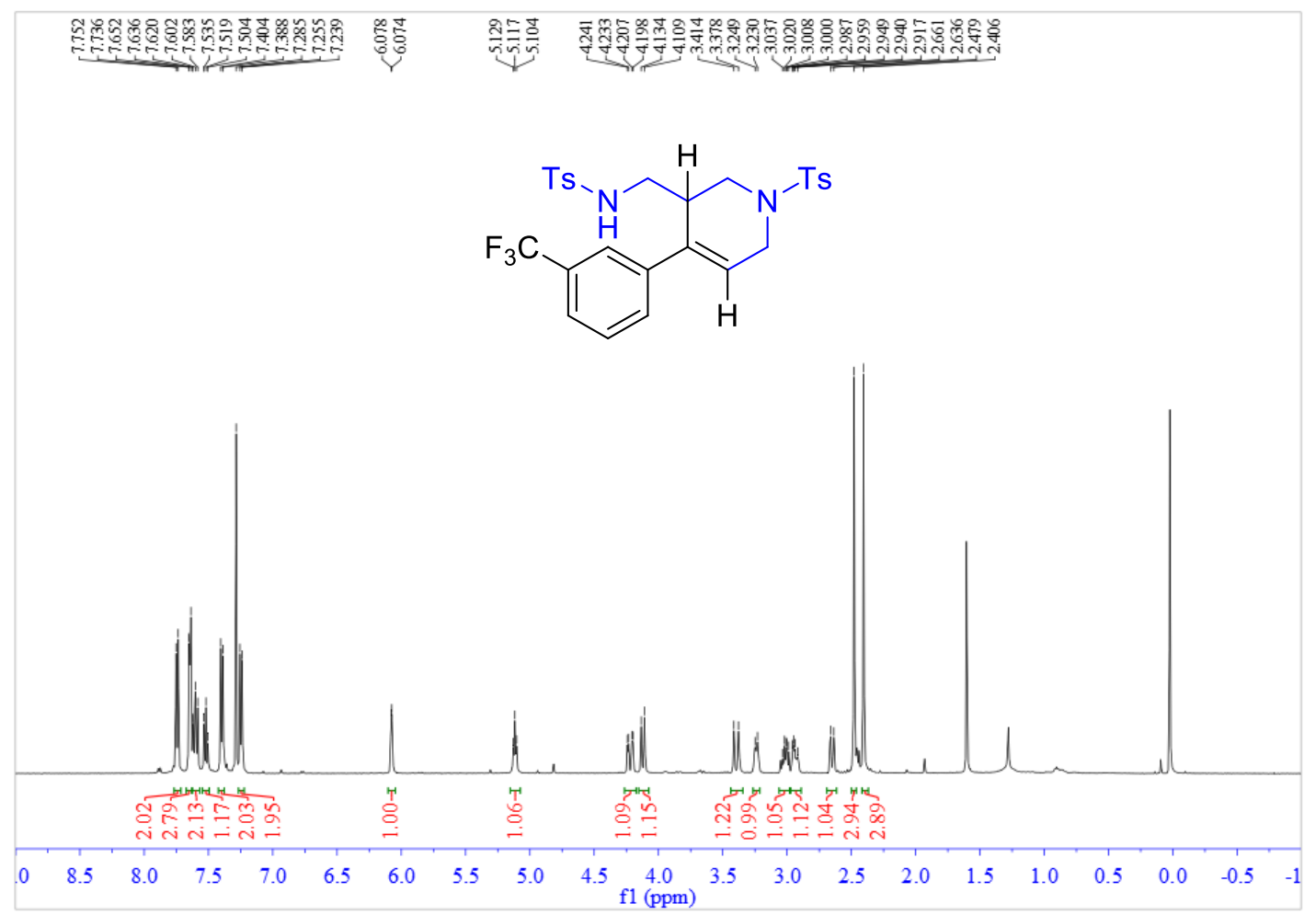

${ }^{13} \mathrm{C}$ NMR (125 MHz, $\mathrm{CDCl}_{3}$ ) of compound $\mathbf{6 m}$

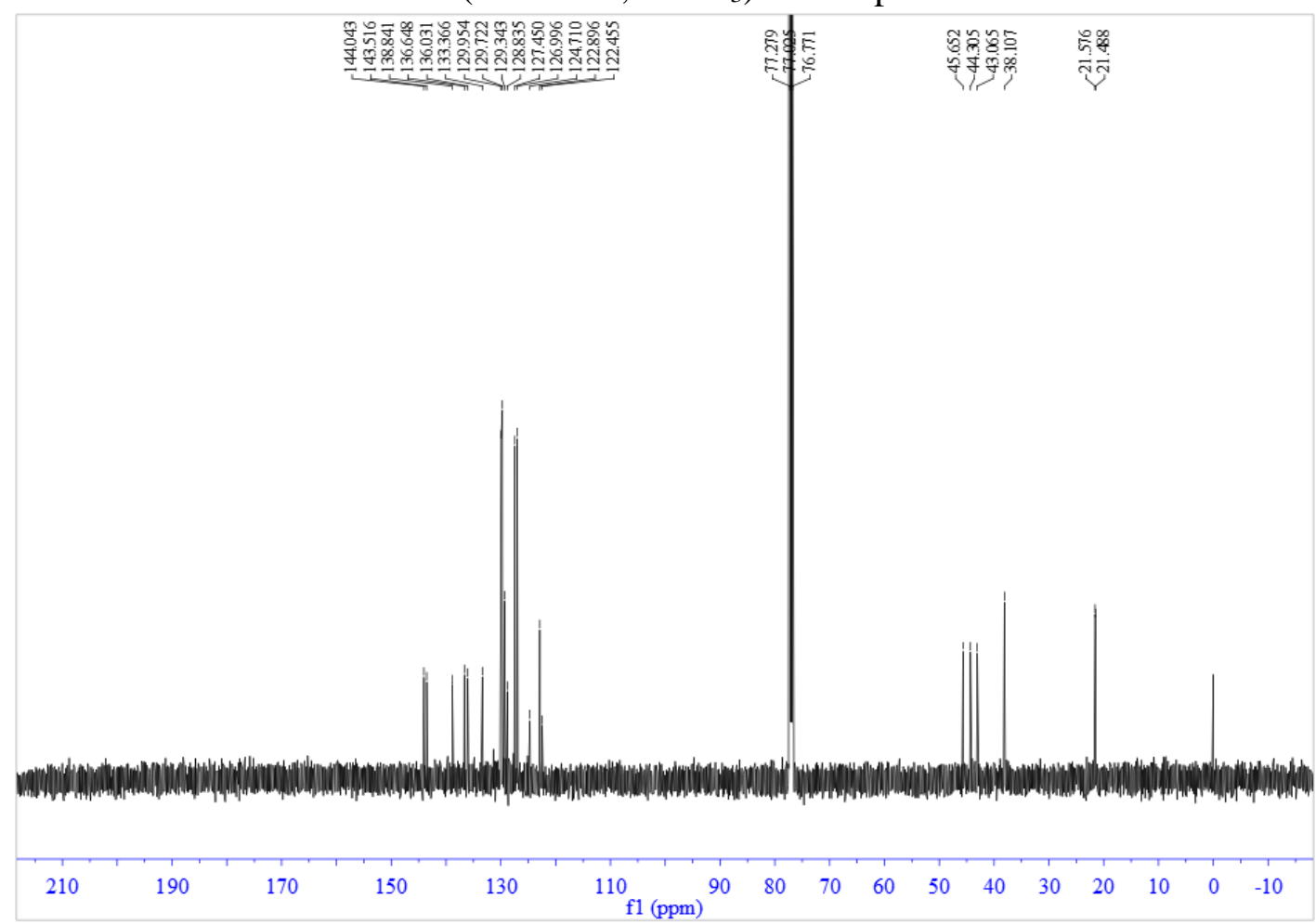


${ }^{19} \mathrm{~F}$ NMR (470 MHz, $\mathrm{CDCl}_{3}$ ) of compound $\mathbf{6 m}$

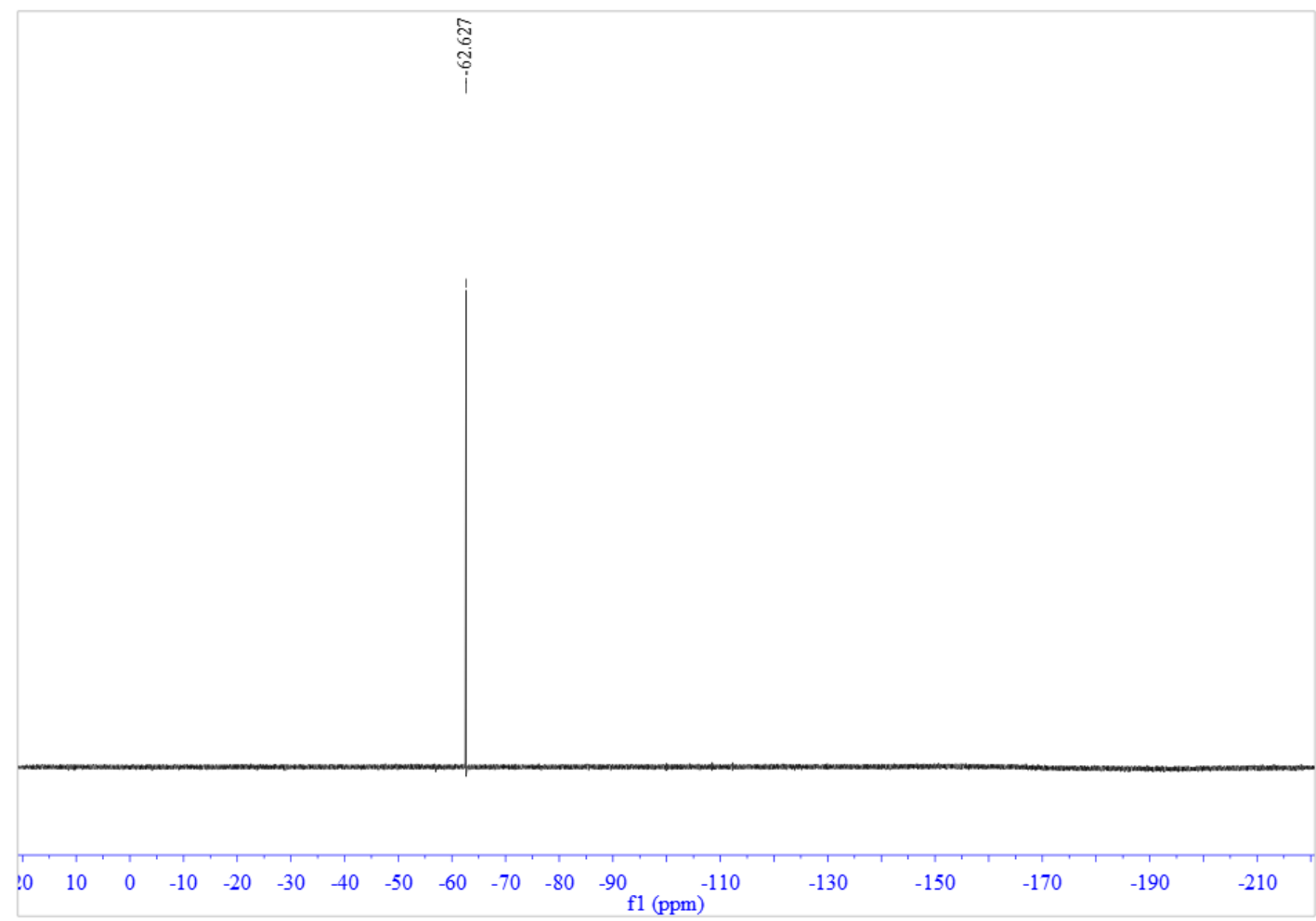


${ }^{1} \mathrm{H}$ NMR $\left(500 \mathrm{MHz}, \mathrm{CDCl}_{3}\right)$ of compound $\mathbf{6 n}$

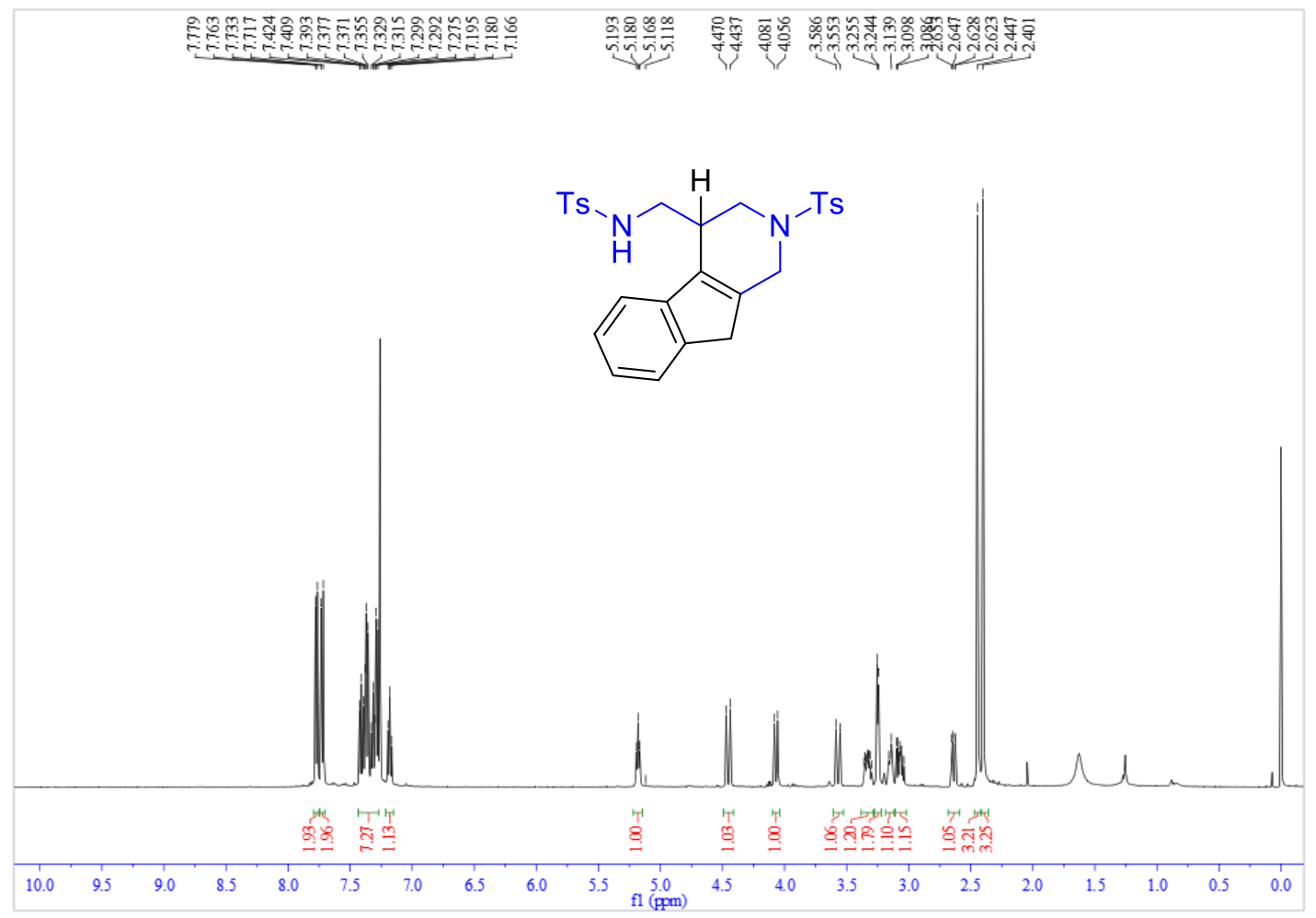

${ }^{13} \mathrm{C}$ NMR $\left(125 \mathrm{MHz}, \mathrm{CDCl}_{3}\right)$ of compound $\mathbf{6 n}$

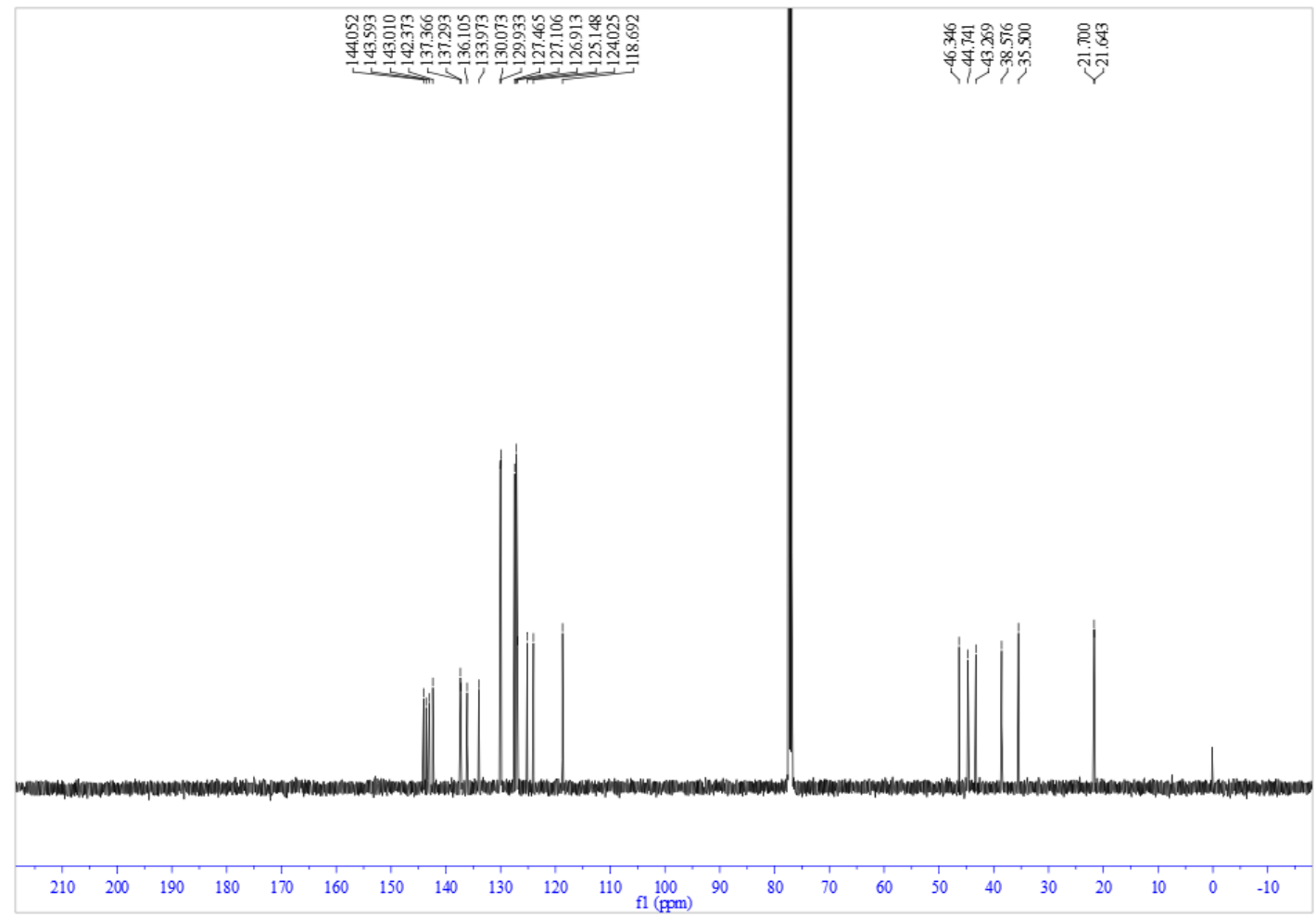


${ }^{1} \mathrm{H}$ NMR $\left(500 \mathrm{MHz}, \mathrm{CDCl}_{3}\right.$ ) of compound 7a

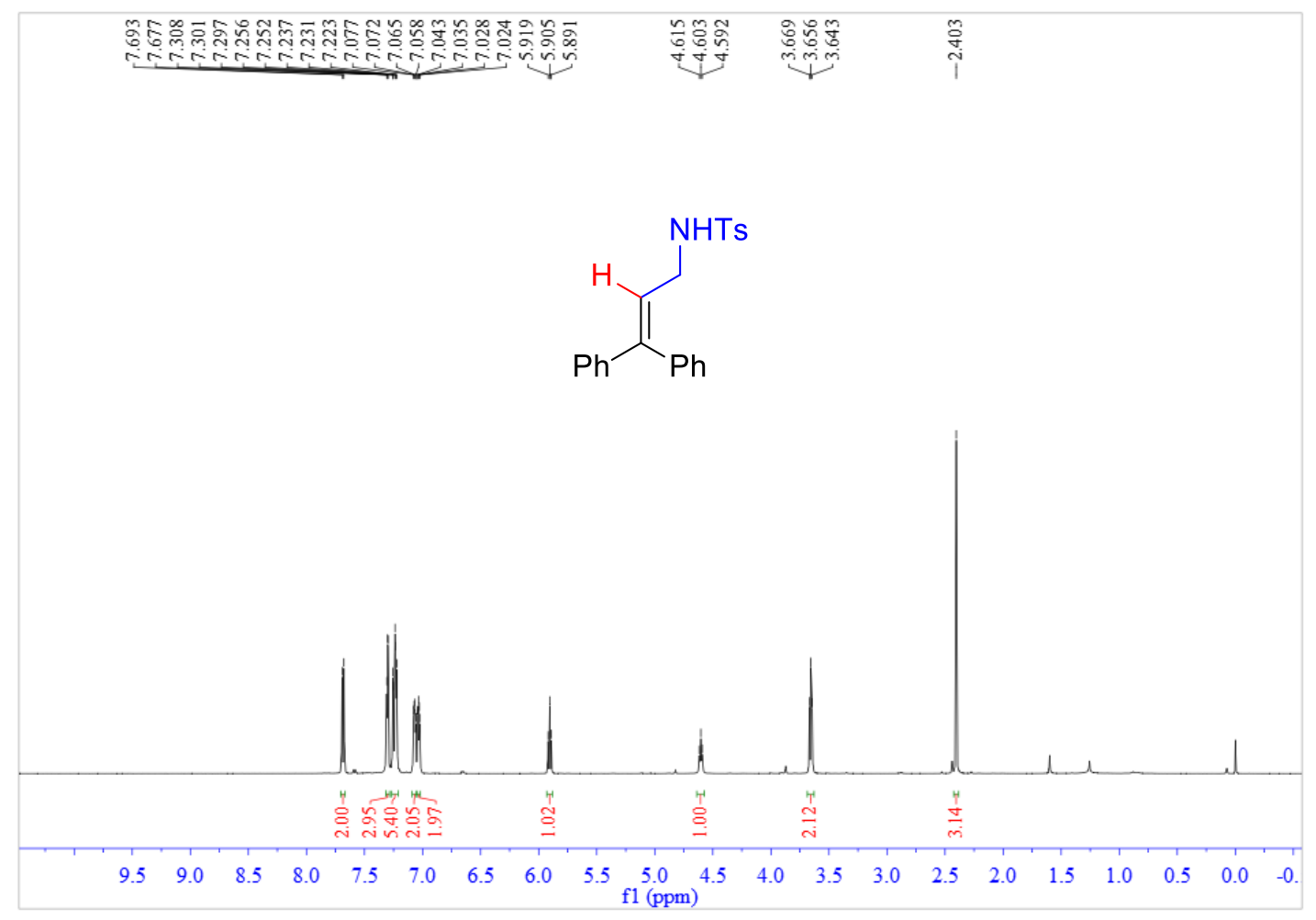

${ }^{13} \mathrm{C}$ NMR (125 MHz, $\mathrm{CDCl}_{3}$ ) of compound 7a

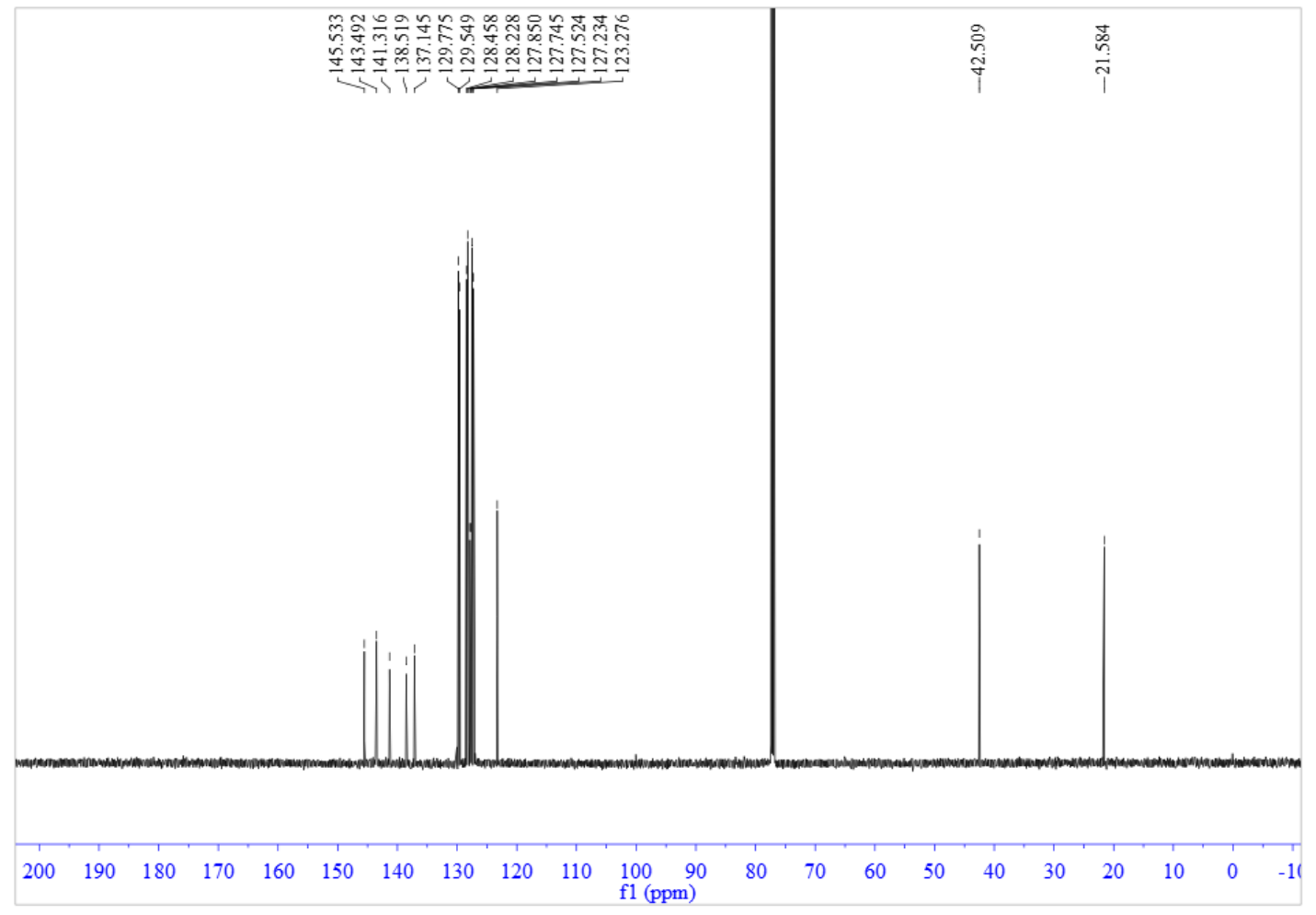




\section{${ }^{1} \mathrm{H}$ NMR $\left(500 \mathrm{MHz}, \mathrm{CDCl}_{3}\right)$ of compound $\mathbf{8 a}$}

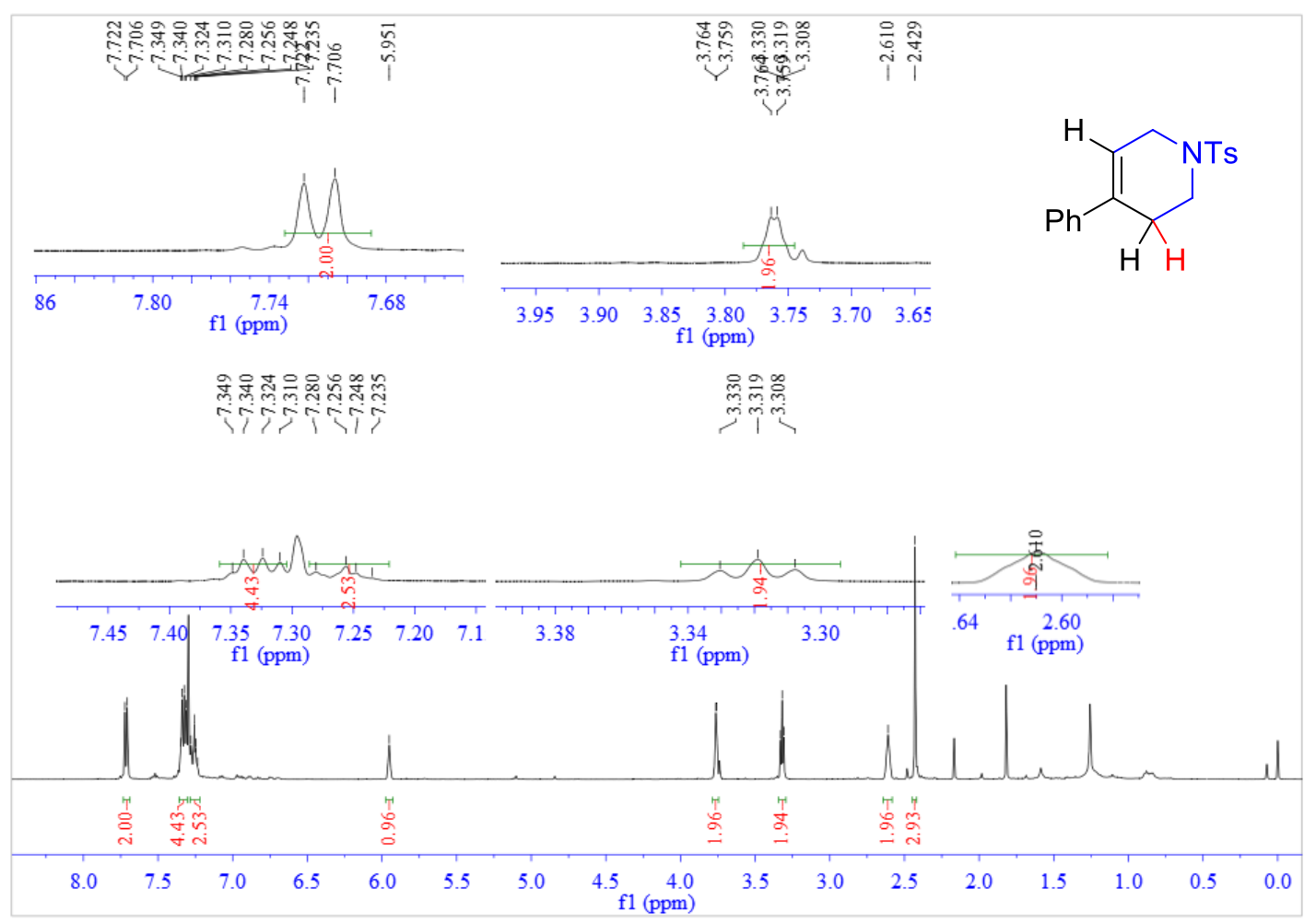

${ }^{13} \mathrm{C}$ NMR $\left(125 \mathrm{MHz}, \mathrm{CDCl}_{3}\right)$ of compound $\mathbf{8 a}$

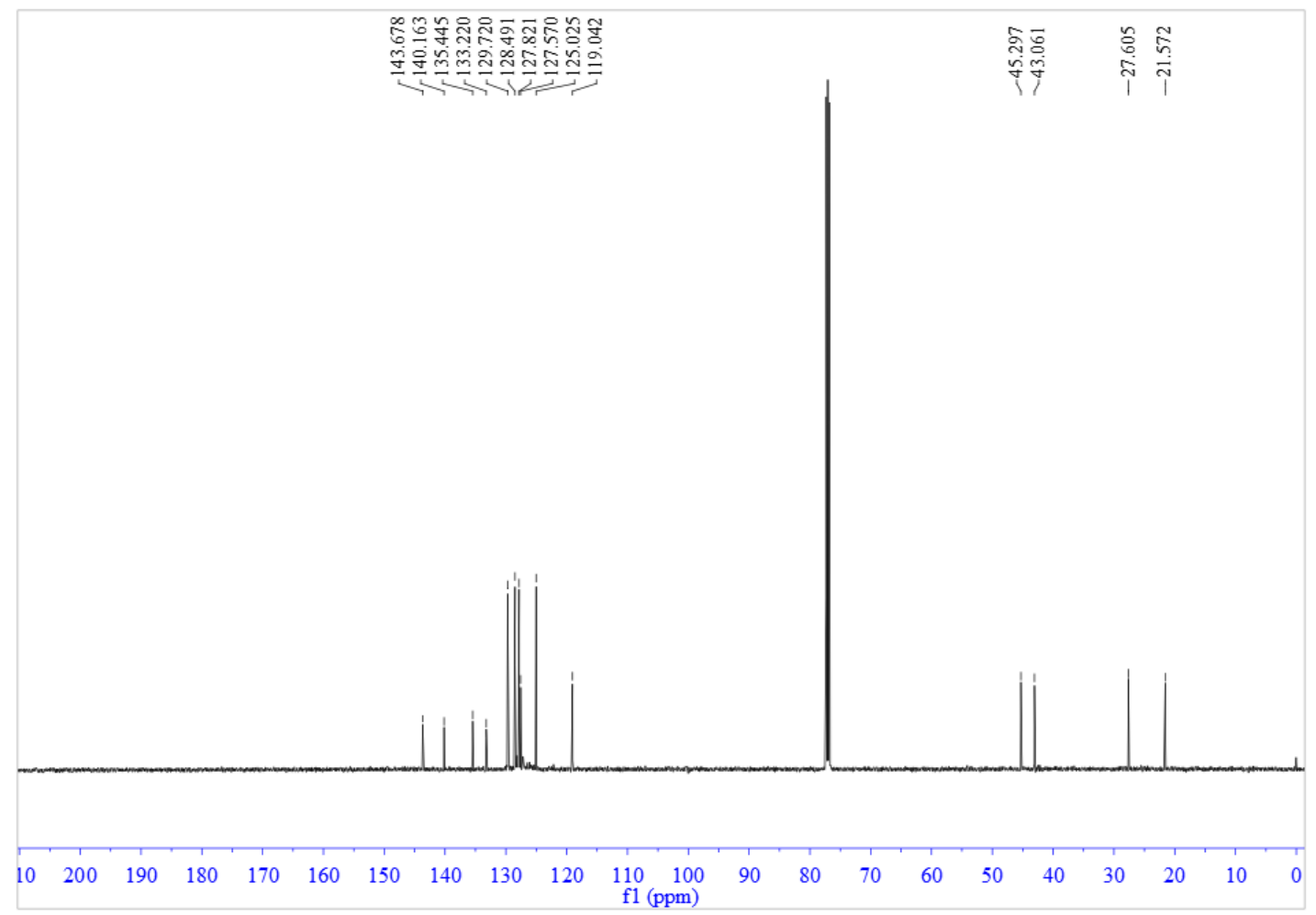




\section{${ }^{1} \mathrm{H}$ NMR $\left(500 \mathrm{MHz}, \mathrm{CDCl}_{3}\right)$ of compound $8 \mathbf{n}$}

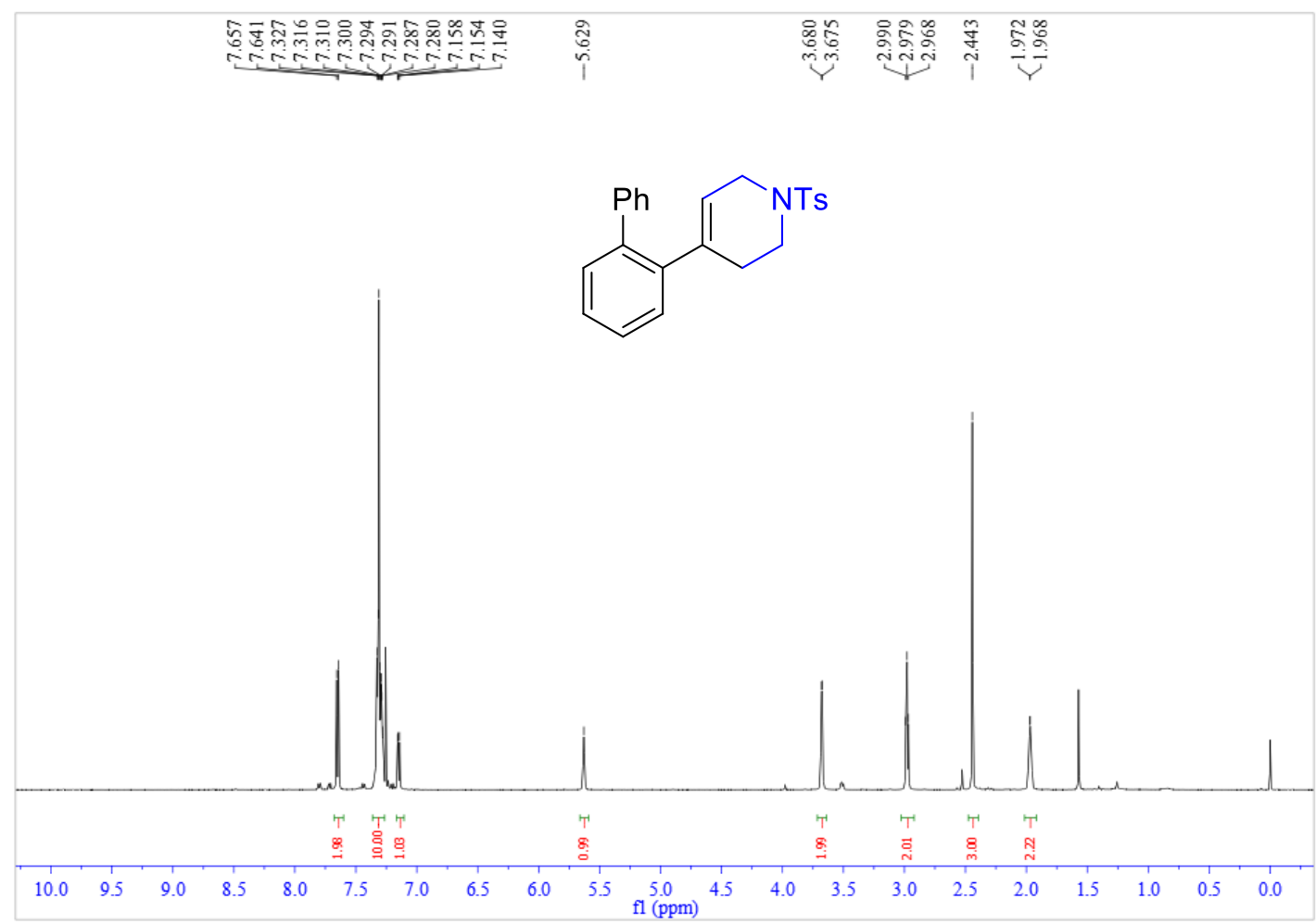

${ }^{13} \mathrm{C}$ NMR (125 MHz, $\mathrm{CDCl}_{3}$ ) of compound $8 \mathbf{n}$

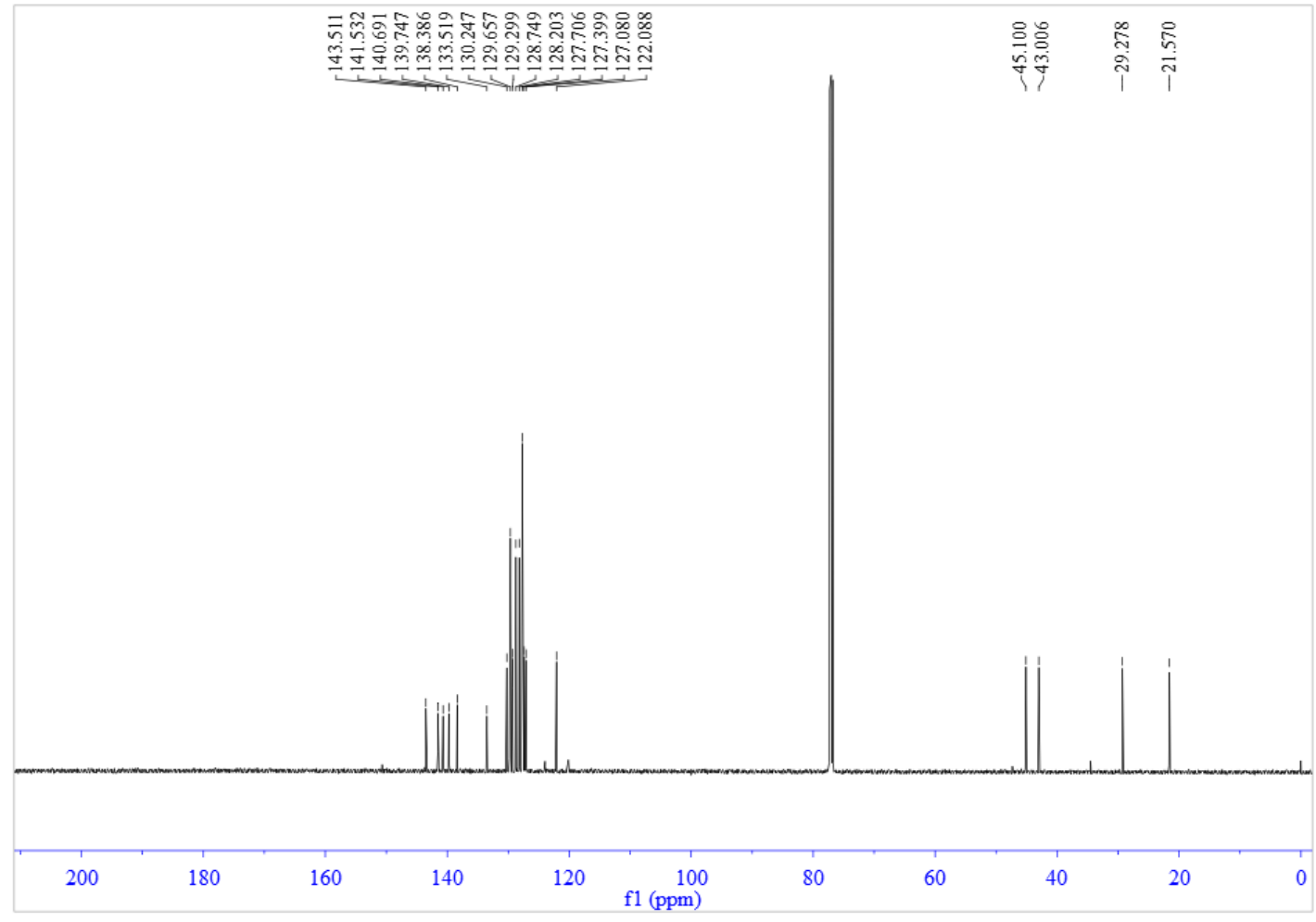


${ }^{1} \mathrm{H}$ NMR (500 MHz, $\mathrm{CDCl}_{3}$ ) of compound 11

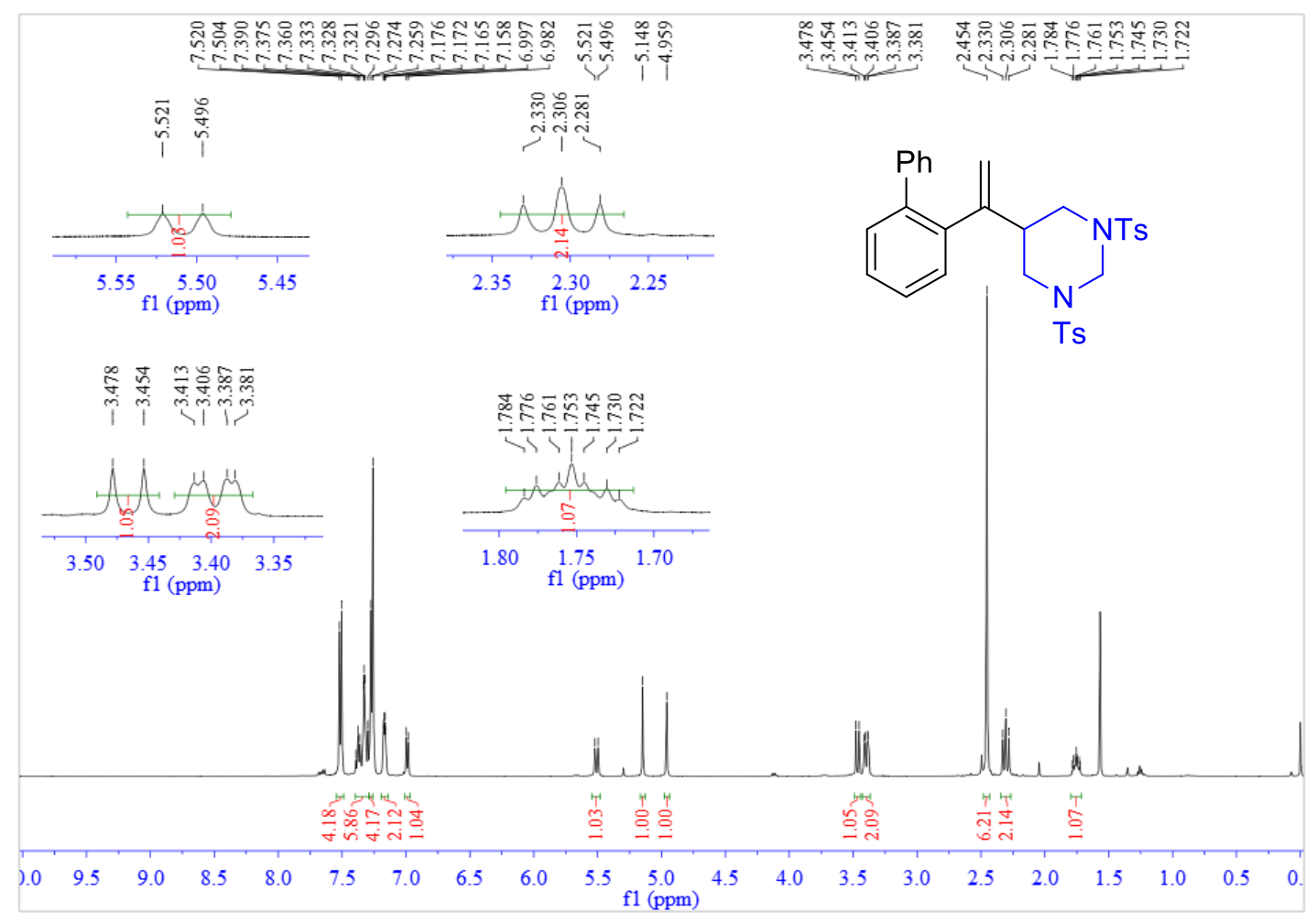

${ }^{13} \mathrm{C}$ NMR (125 MHz, $\mathrm{CDCl}_{3}$ ) of compound $\mathbf{1 1}$

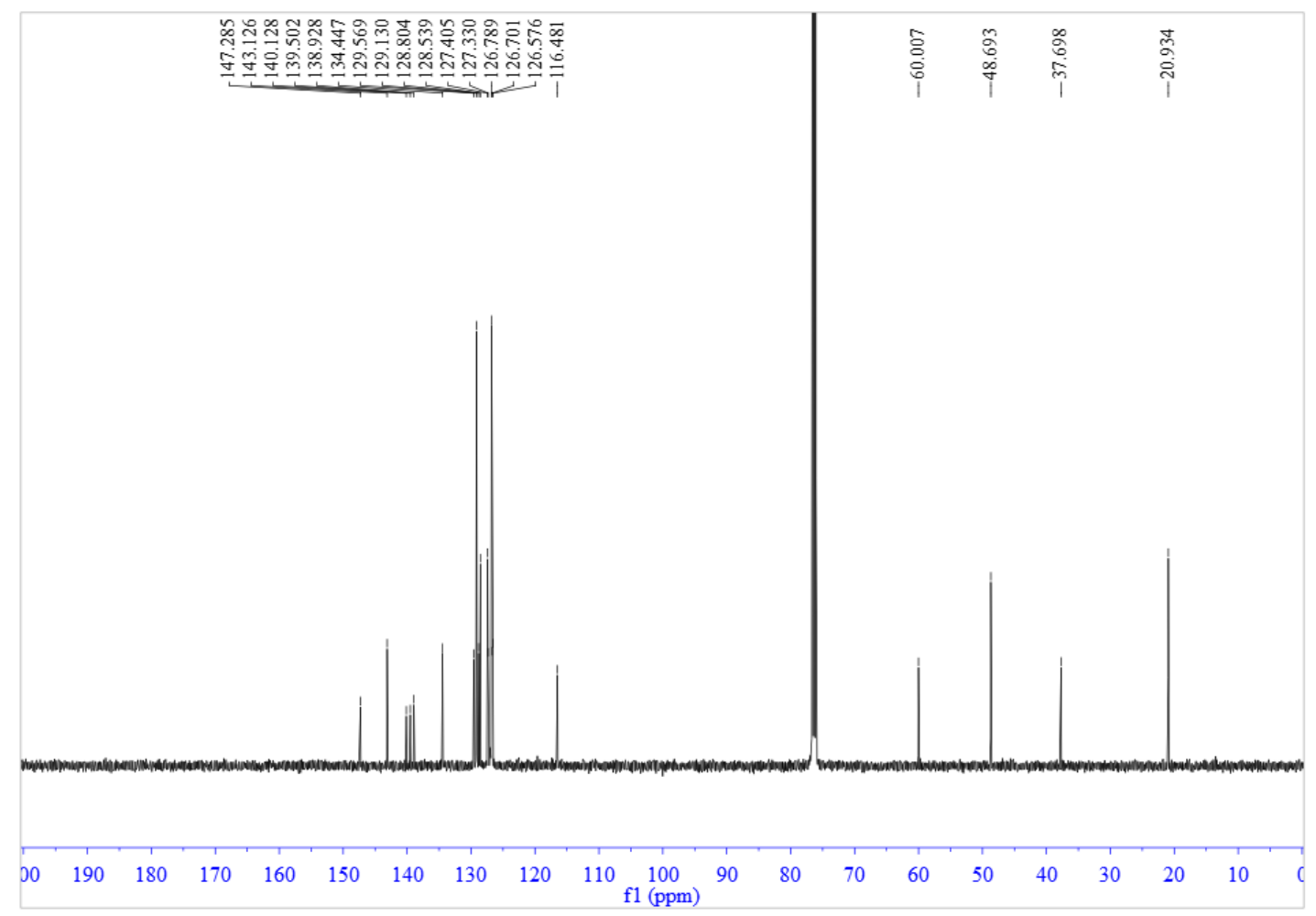


${ }^{1} \mathrm{H}$ NMR (500 MHz, $\mathrm{CDCl}_{3}$ ) of compound 12a

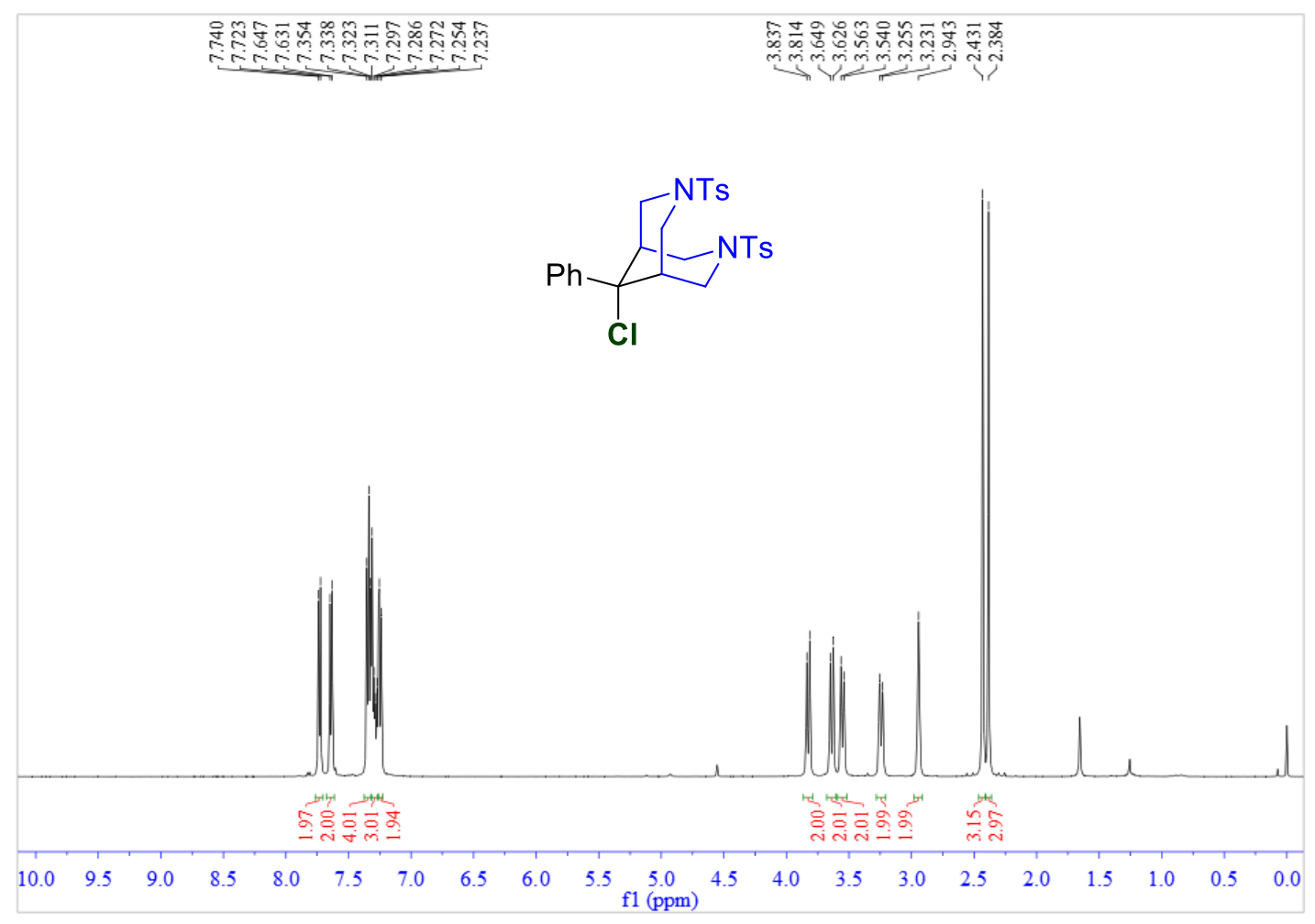

${ }^{13} \mathrm{C}$ NMR $\left(125 \mathrm{MHz}, \mathrm{CDCl}_{3}\right)$ of compound 12a

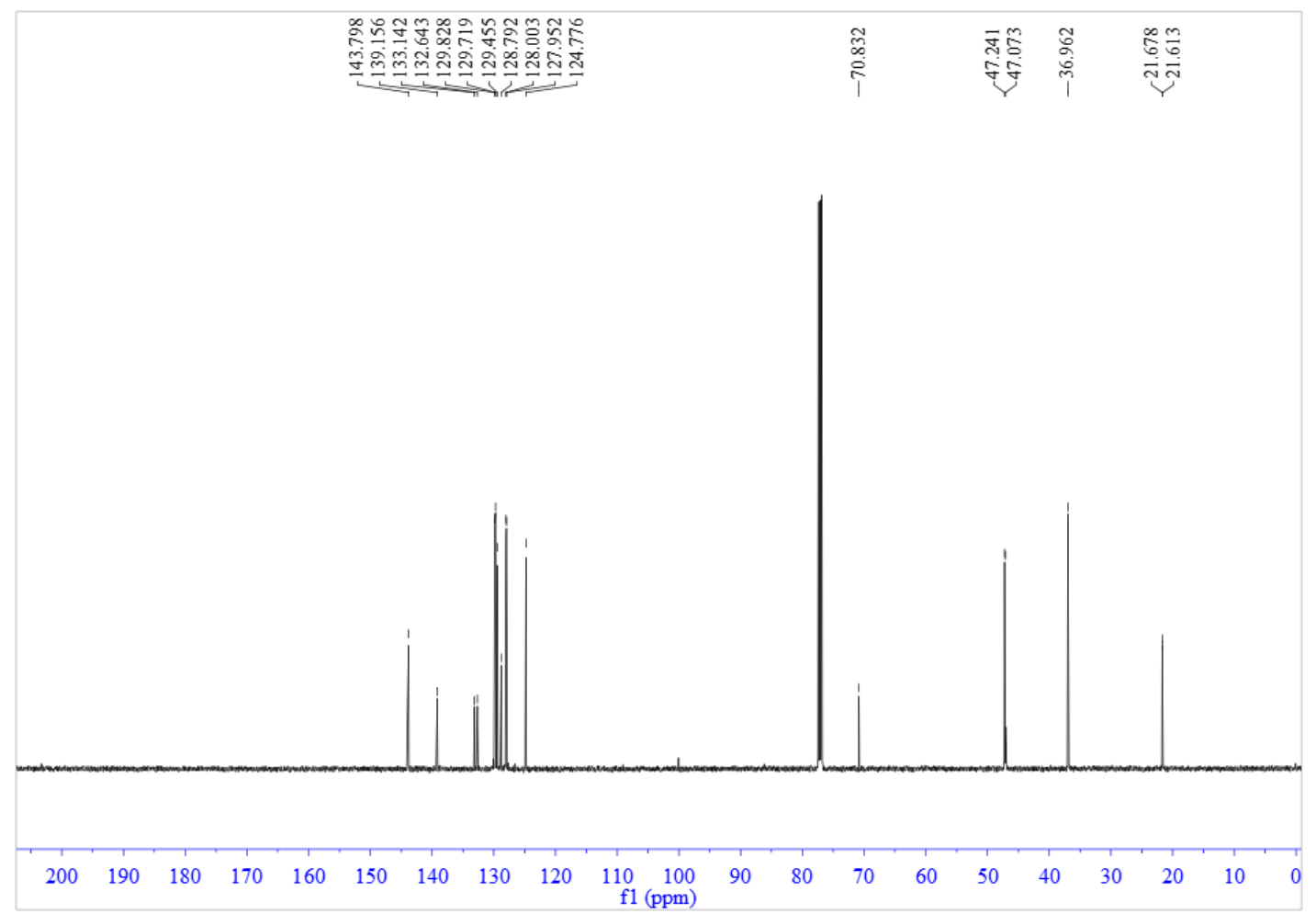


${ }^{13} \mathrm{C}$ APT of compound $\mathbf{1 2 a}$

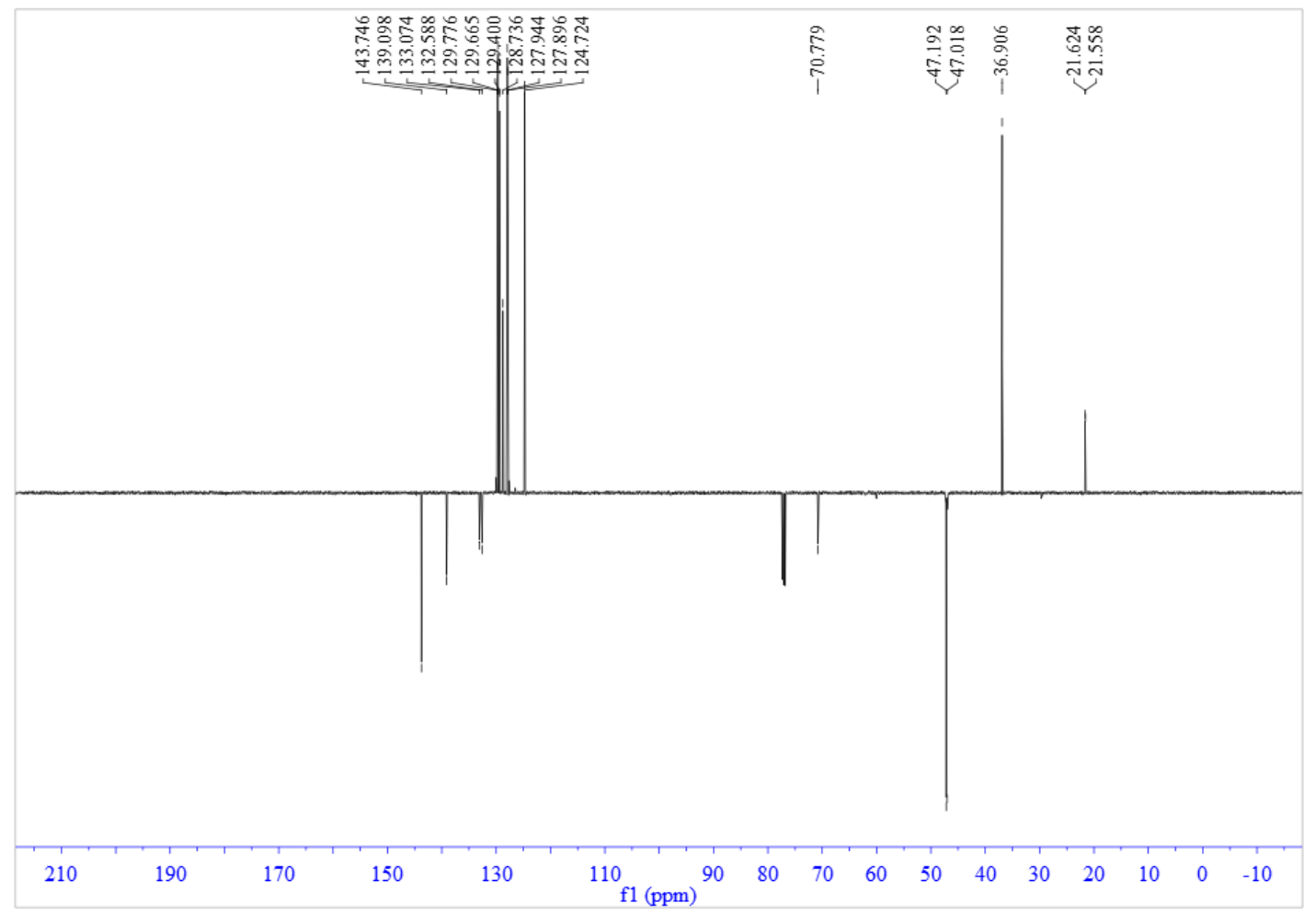


${ }^{1} \mathrm{H}$ NMR (500 MHz, $\left.\mathrm{CDCl}_{3}\right)$ of compound $\mathbf{1 2 b}$

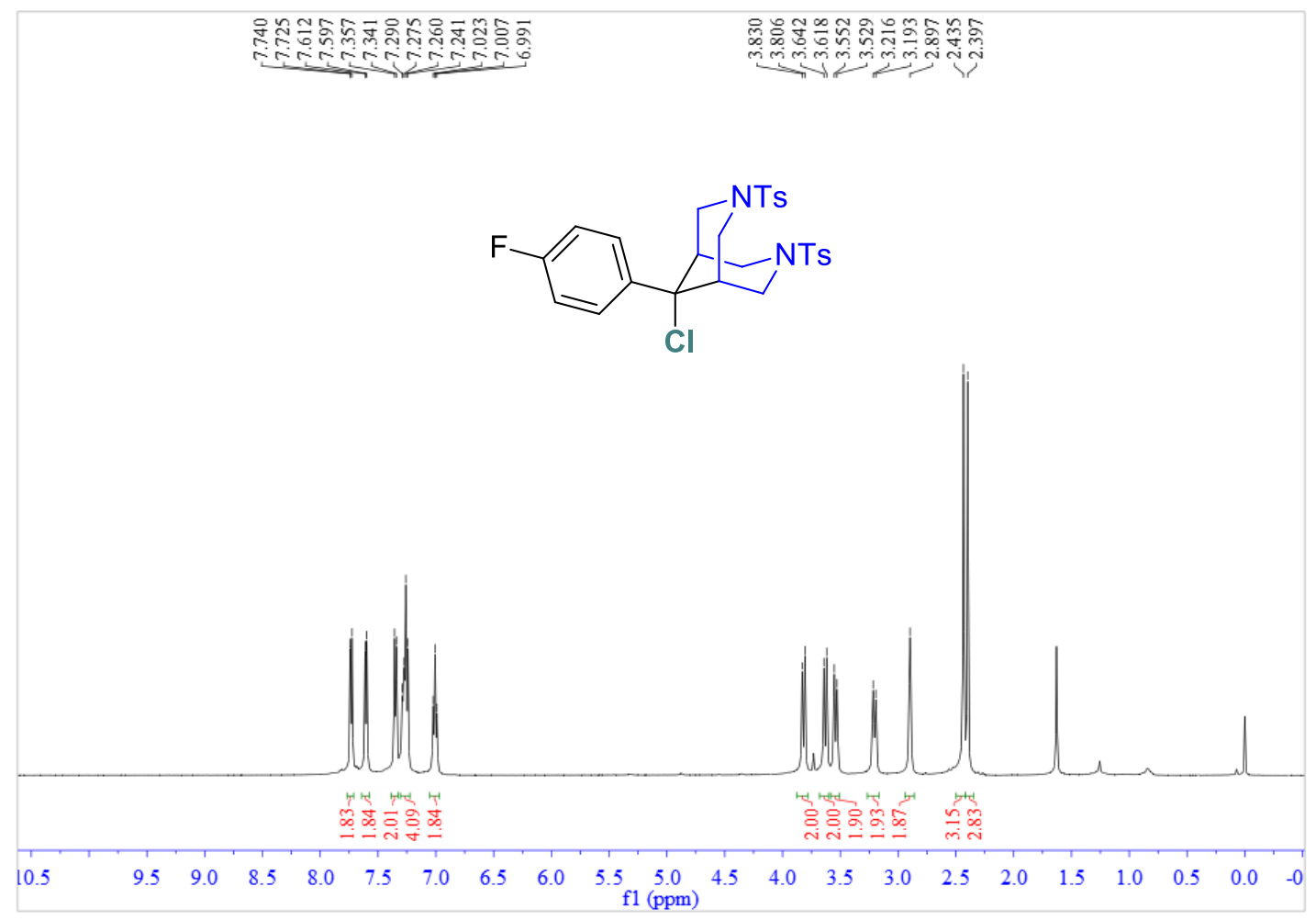

${ }^{13} \mathrm{C}$ NMR (125 MHz, $\mathrm{CDCl}_{3}$ ) of compound $\mathbf{1 2 b}$

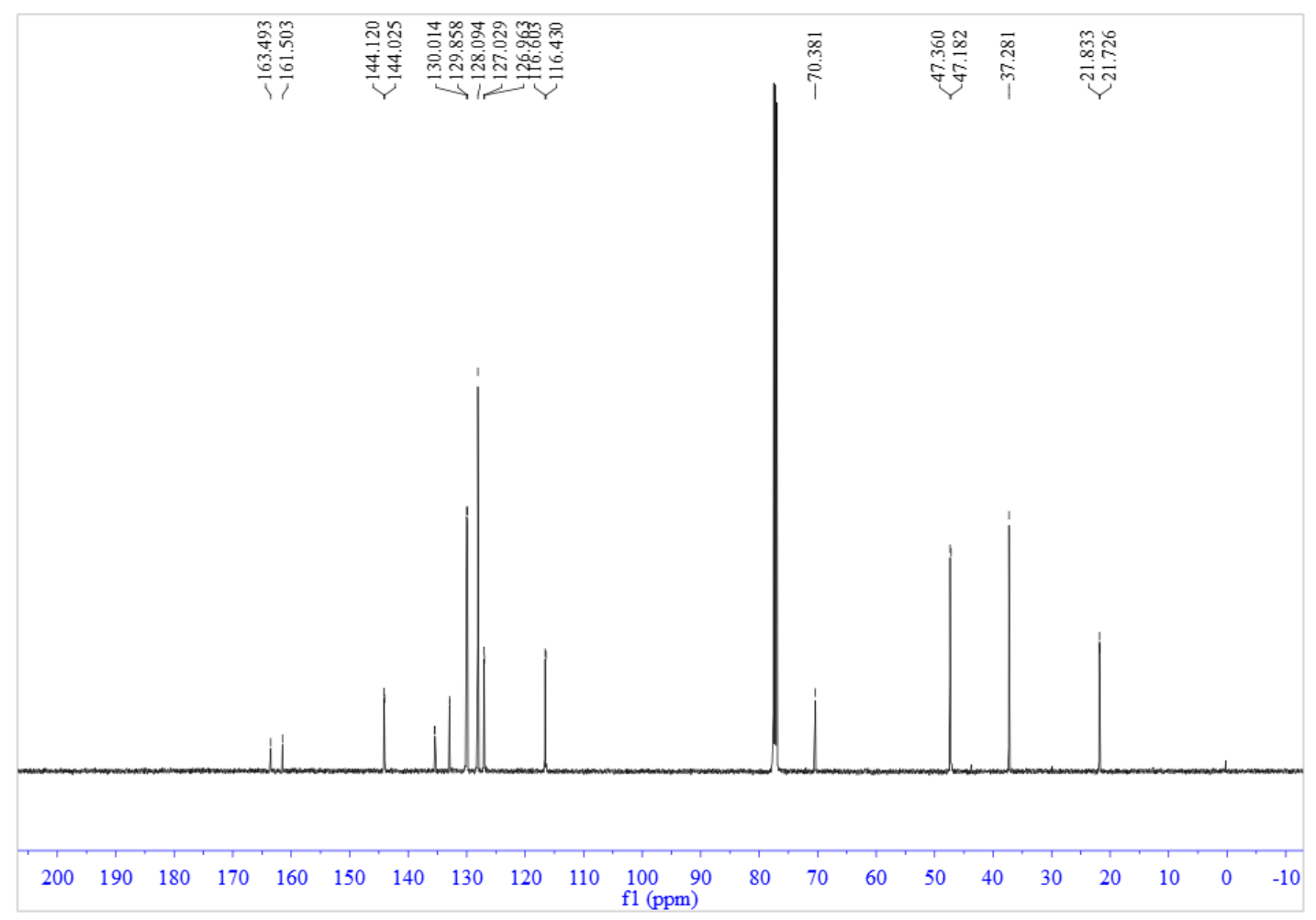


${ }^{19} \mathrm{~F}$ NMR $\left(470 \mathrm{MHz}, \mathrm{CDCl}_{3}\right)$ of compound $\mathbf{1 2 b}$

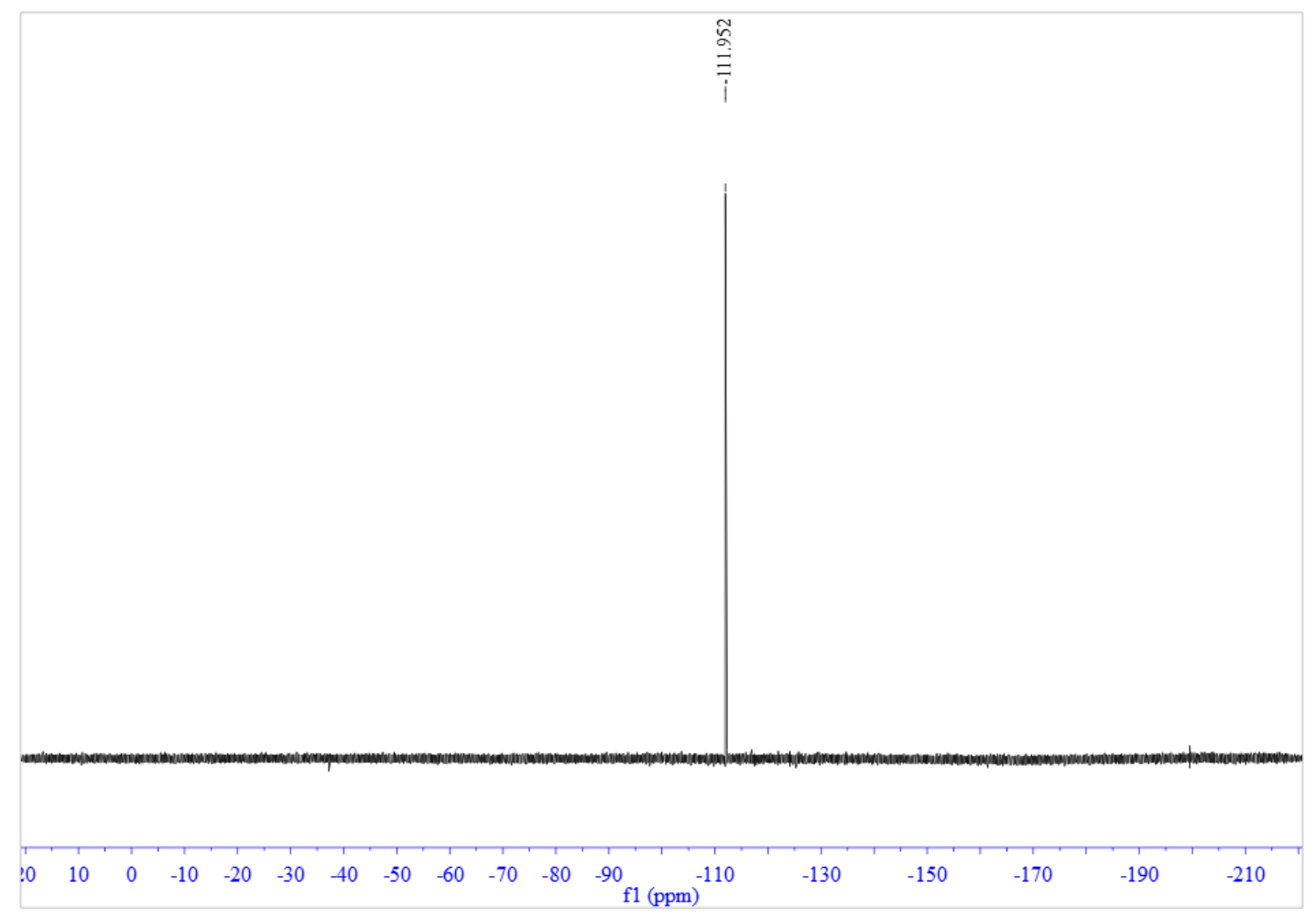


${ }^{1} \mathrm{H}$ NMR $\left(500 \mathrm{MHz}, \mathrm{CDCl}_{3}\right.$ ) of compound $\mathbf{1 2 c}$

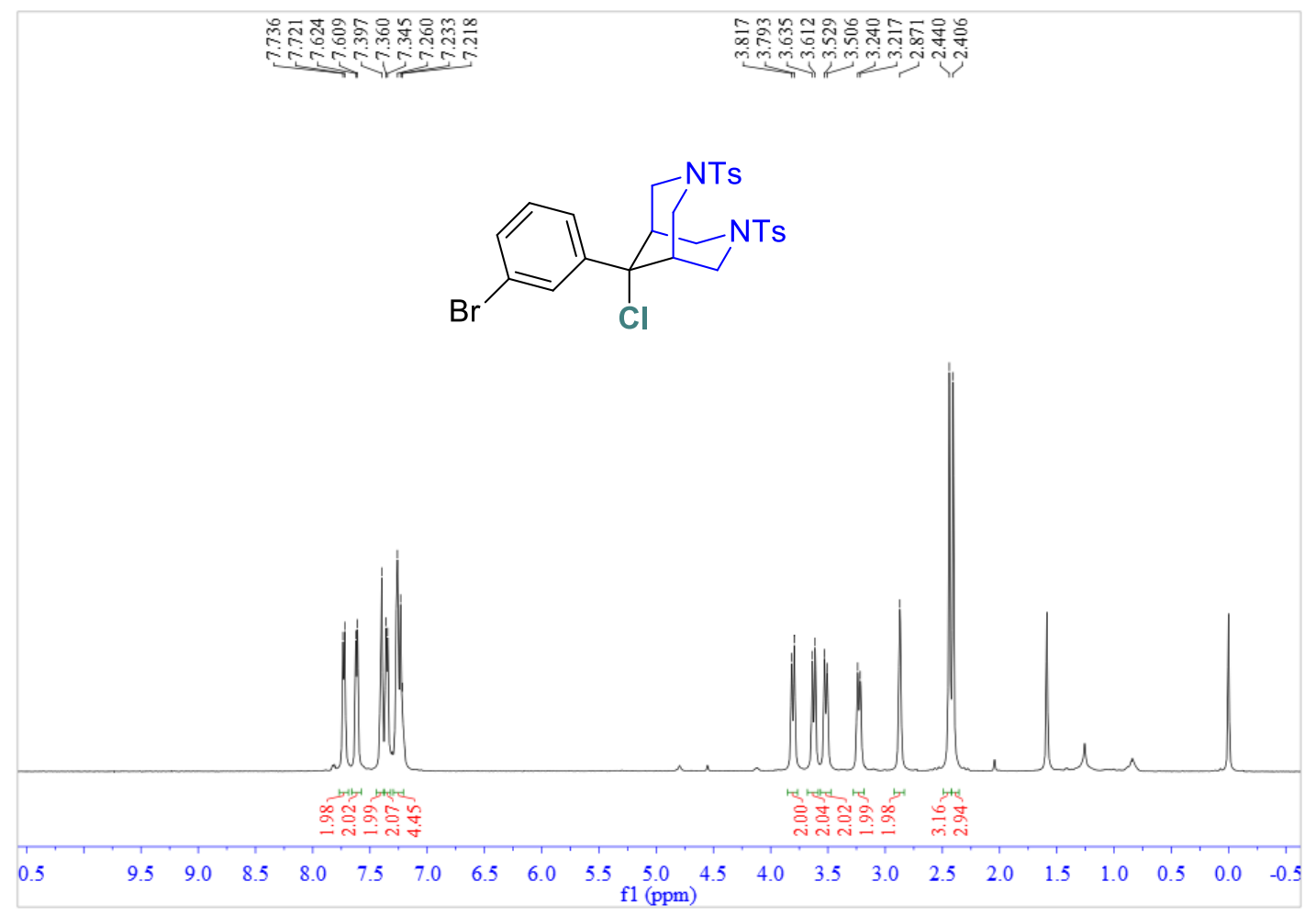

${ }^{13} \mathrm{C}$ NMR $\left(125 \mathrm{MHz}, \mathrm{CDCl}_{3}\right.$ ) of compound $\mathbf{1 2 c}$

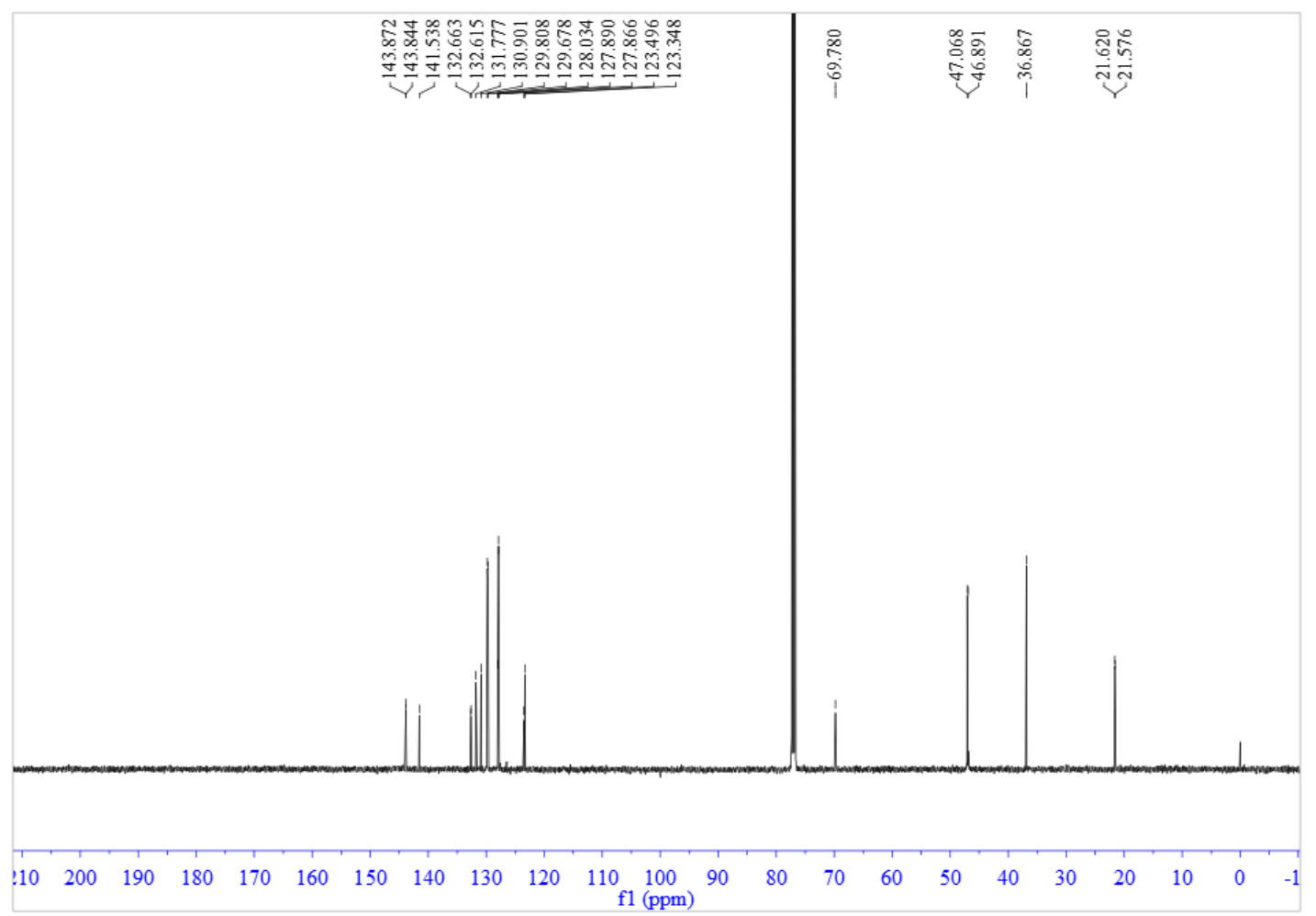

